University of Louisville

ThinkIR: The University of Louisville's Institutional Repository

Electronic Theses and Dissertations

$12-2012$

\title{
Improved fabrication and modeling of piezoresistive microcantilever beams for gas detection and sensing.
}

Ni Wang

University of Louisville

Follow this and additional works at: https://ir.library.louisville.edu/etd

\section{Recommended Citation}

Wang, Ni, "Improved fabrication and modeling of piezoresistive microcantilever beams for gas detection and sensing." (2012). Electronic Theses and Dissertations. Paper 1520.

https://doi.org/10.18297/etd/1520

This Doctoral Dissertation is brought to you for free and open access by ThinkIR: The University of Louisville's Institutional Repository. It has been accepted for inclusion in Electronic Theses and Dissertations by an authorized administrator of ThinkIR: The University of Louisville's Institutional Repository. This title appears here courtesy of the author, who has retained all other copyrights. For more information, please contact thinkir@louisville.edu. 


\title{
IMPROVED FABRICATION AND MODELING OF PIEZORESISTIVE MICROCANTILEVER BEAMS FOR GAS DETECTION AND SENSING
}

\author{
By \\ Ni Wang \\ B.S., Wuhan Transportation Technology University, Wuhan, China, 2001 \\ M.S., University of Louisville, Louisville, KY, USA 2007

\begin{abstract}
A Dissertation
Submitted to the Faculty of the

Speed School of Engineering of University of Louisville

In Partial Fulfillment of the Requirements

For the Degree of
\end{abstract}

Doctor of Philosophy

Department of Mechanical Engineering

University of Louisville

Louisville, Kentucky

December 2012 


\title{
IMPROVED FABRICATION AND MODELING OF PIEZORESISTIVE MICROCANTILEVER BEAMS FOR GAS DETECTION AND SENSING
}

\author{
By \\ Ni Wang \\ B.S., Wuhan Transportation Technology University, Wuhan, China, 2001 \\ M.S., University of Louisville, Louisville, KY, USA 2007
}

A Dissertation Approved on

December 5, 2012

By the following Dissertation Committee:

Dr. Roger D. Bradshaw, Dissertation Director, Department of Mechanical Engineering

Dr. Thomas A. Berfield, Department of Mechanical Engineering

Dr. Robert S. Keynton, Department of Bioengineering

Dr. Yongsheng Lian, Department of Mechanical Engineering

Dr. Christopher M. Richards, Department of Mechanical Engineering 


\section{DEDICATION}

This dissertation is dedicated to my family! 


\section{ACKNOWLEDGMENTS}

I want to express my most sincere appreciation to my Ph.D. advisor, Dr. Roger D. Bradshaw, for his intelligent supervision, constructive guidance, inspiration and patience. His perpetual energy and enthusiasm in research has motivated all his advisees, including me. In addition, he was always accessible and willing to help his students with their research. He is not only the academic role model for me but also a role model of my personal life. Dr. Bradshaw is and will remain my best role model for a scientist, mentor, and teacher.

My sincere appreciation extends to other committee members: Dr. Keynton for his generous financial support for my work in the cleanroom and guidance in questions of microfabrication; Dr. Richards for his assistance with measurements in the lab; Dr. Lian for insightful discussion related to CFD analysis; and Dr. Berfield for his the knowledge of microfabrication. Moreover, I wish to express my sincere gratitude to Dr. Alphenaar, who let me work in his lab and use his equipment for this study, and also for offering valuable suggestions and assistance regarding experimental measurements in study. Thanks are also due to Mr. Mark Crain and Dr. Patrick Fletcher for providing the assistance in cleanroom.

I would also like to thank Dr. Glen Prater and the Department of Mechanical Engineering for financial support, including cleanroom fees during the fabrication process. This work was also supported by a Multidisciplinary Research Grant from the 
University of Louisville. Finally, my degree would not have been possible without the Grosscurth fellowship I received as I began my Ph.D studies.

I would also like to thank John Jones, Sue Jones, Dianne Jenne and Brent Hudson of the Department of Mechanical Engineering, Julia Aebersold and Don Yeager of the Micro Nano Technology Center (MNTC) and Tanesh and Huihang Dong. All your help makes every progress of my study possible.

My deepest gratitude goes to my family for their unflagging love and support throughout my life; this dissertation is simply impossible without them. I am indebted to my parents, Wang and Chen, for their care and love. As typical parents in a Chinese family, they spare no effort to provide the best possible environment for me to grow up and pursue academic advancement.

I want to give my special appreciation to my husband, Qiang He, for his support to my study, his encouragement at times of difficulty, and his love and understanding throughout my study. Thanks also go to my friends for their support and encouragement. 


\section{ABSTRACT \\ IMPROVED FABRICATION AND MODELING OF PIEZORESISTIVE MICROCANTILEVER BEAMS FOR GAS DETECTION AND SENSING}

\section{Ni Wang}

December 5, 2012

Symmetric piezoresistive microcantilever beams have been demonstrated in previous research to be capable of sensing the presence of surrounding gas. This occurs as the damping effect of the gas changes the beam resonance behavior. Device sensitivity has been increased dramatically after changing the symmetric beam base to an asymmetric beam base.

This dissertation seeks to improve on beam fabrication and simplify the fabrication procedure compared to earlier approaches. By changing to a polymer mask and using new equipment, an entire wafer can be fabricated in far less time compared to the previous approach. While this new approach shows great promise, additional research is needed to demonstrate consistent device quality comparable with earlier approaches. This dissertation also focuses on the continued development of such devices with an emphasis on modeling to better understand the resonant behavior in a gas. Past work at the University of Louisville and elsewhere has relied upon simplified fluid mechanics models to relate changes in resonance with gas properties. The current work considers a combination of Stokes' oscillating cylinder model and computational fluid dynamics simulation to better characterize the damping effect including the effect of the rectangular 
cross-section and the presence of a boundary (the silicon handle layer) located $2 \mu \mathrm{m}$ below the beam.

The beam is induced to vibrate by electrical attractive forces at the end that change with applied driving electrode voltage and beam voltage. The electrostatic force, the displacement of the beam tip, the change of resistance of beam base due to piezoresistive effects, and the resulting signal received by the lock-in amplifier is established by a combination of analytical models and finite element simulation. This simulated output signal provides valuable insights to address issues of proper parameters to use during testing.

This new information developed in this dissertation helps to advance the state of the art for microresonating beams for gas detection. This information is expected to play a key role as the systems in this work are transitioned to use in practice. 


\section{TABLE OF CONTESTS}

DEDICATION ............................................................................................................

ACKNOWLEDGMENTS .............................................................................................. IV

ABSTRACT ...................................................................................................................... VI

TABLE OF CONTESTS ............................................................................................. VIII

LIST OF FIGURES ...................................................................................................................XII

LIST OF TABLES .....................................................................................................XXI

CHAPTER 1. INTRODUCTION ..........................................................................................

1.1 BACKGROUND REVIEW ........................................................................... 2

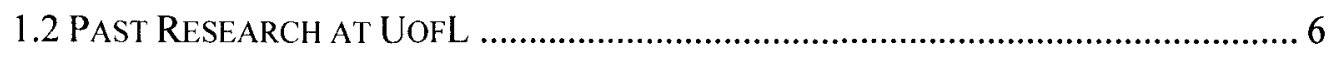

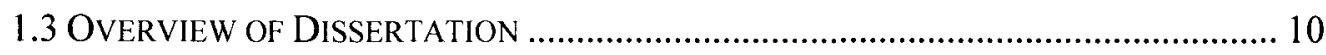

CHAPTER 2. DEVICE FABRICATION AND OPERATION .....................................12

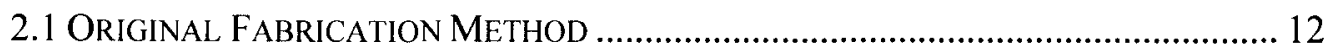

2.2 IMPROVED FABRICATION METHOD - DETAILS ................................................... 15

2.3 IMPROVED FABRICATION METHODS - RESULtS ................................................. 19

2.4 IMPROVED FABRICATION METHODS -DISCUSSION ……..................................... 21

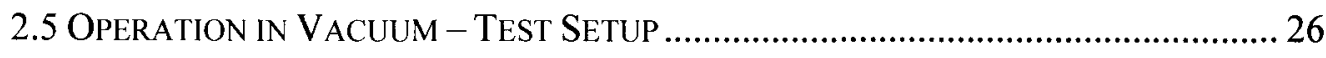

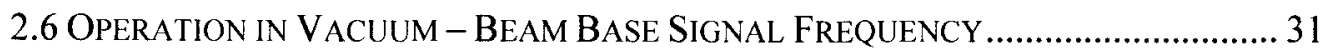


2.7 Operation in VacuUm - Beam BaSe / Driving Electrode Leakage .......... 38

2.8 OPERATION IN VACUUM - AVOIDANCE OF STICTION ..................................... 42

\section{CHAPTER 3. EFFECT OF SUUROUNDING FLUID ON BEAM BEHAVIOR .....43}

3.1 OVERVIEW OF Vibrating MicrocantILEVER IN A GAS ENVIRONMENT ........... 43

3.2 CFX - OVERVIEW AND VALIDATION USing TANEDA RESUltS..........................49

3.2.1 Overview of Taneda Experiments ...............................................49

3.2.2 Setup in CFX - Two Cylinder Experiment .........................................5I

3.2.3 Results - Two Cylinder Experiment ..............................................55

3.2.4 Results - Cylinder Near Wall Experiment ....................................60

3.2.5 Summary - Validation of CFX Using Taneda Experiments .................62

3.3 STUdiES OF 2D BEAM RESONATING IN THE FLUID ..........................................62

3.3.1 Creating Input Geometry ..............................................................63

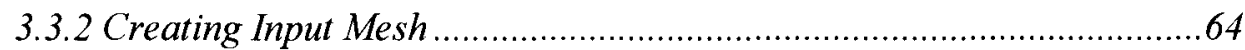

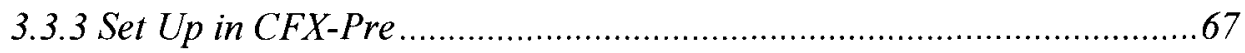

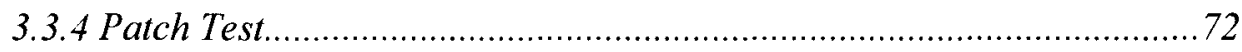

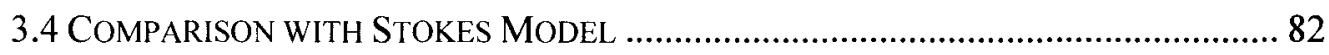

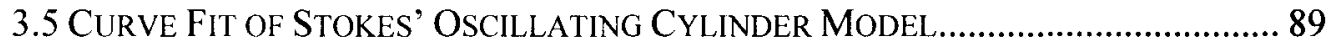

3.6 MicrocantileVER BEAm Fluid Simulation............................................ 97

3.6.1 Cylinder Oscillating Without a Floor .............................................98

3.6.2 Cylinder Oscillating With a Floor ............................................... 99

3.6.3 Rectangle Oscillating Without a Floor ....................................... 102

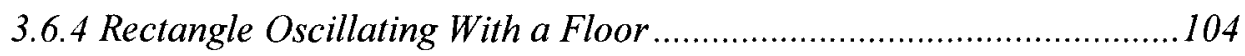

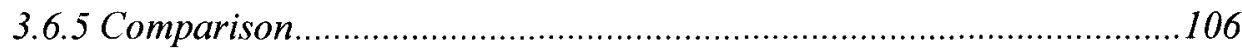


3.6.6 Effect of Different Frequency (Velocity) ........................................ 108

3.6.7 Effect of Shape of Beam Cross Section.............................................111

3.6.8 Viscous Effect of Bottom Wall......................................................112

3.6.9 Comparison to Stokes' Equation ................................................113

3.6.10 Effect of Distance to Floor...................................................116

3.6.11 Summary of Viscosity Loads on Resonating Beam ........................ 120

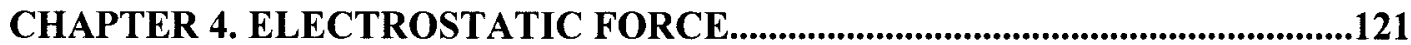

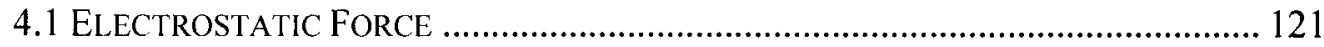

4.2 Modeling OF Electrostatic Beam Tip Forces ....................................... 125

4.3 DRIVING ELECTRODE ForCE MOdEL ......................................................... 131

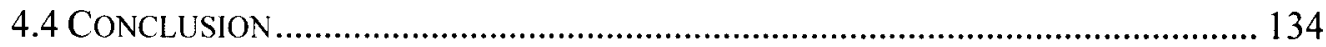

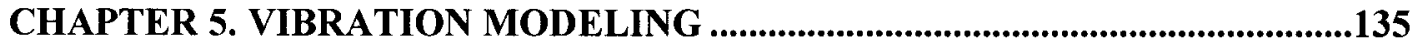

5.1 Model and Governing EQuation of Microcantilever Beam Resonation 135

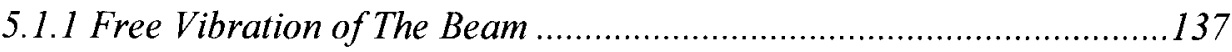

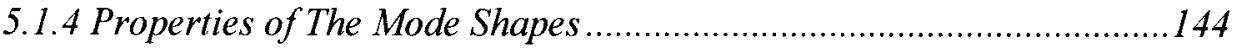

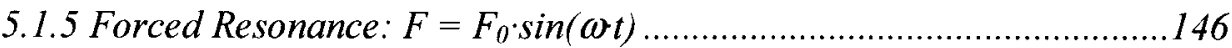

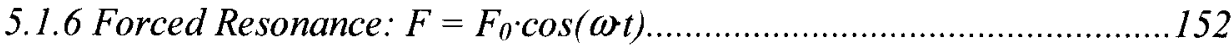

5.1.7 Steady State Solution of Forced Resonance ......................................153

5.2 Device Behavior Under Driving Electrode Actuation ......................... 156

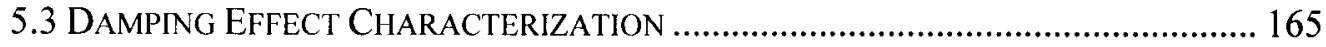

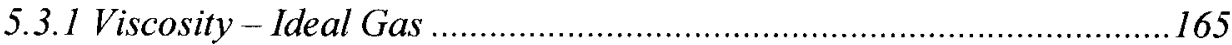


5.3.2 Viscosity - Rarefied Gas and Knudsen Number.............................. 166

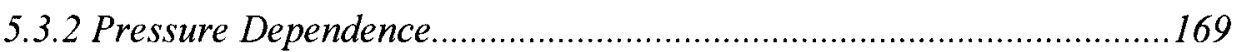

CHAPTER 6. SIMULATION OF SIGNAL AT LOCK-IN AMPLIFIER ...............172

6.1 SUMMARY OF THE RELATIONSHIP DEVELOPED ......................................... 172

6.2 SimUlation OF tHE SigNAL TO THE LOCK-IN AMPLIFIER ............................ 173

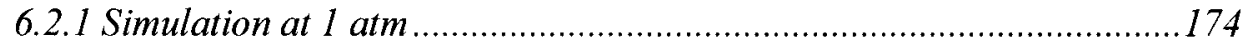

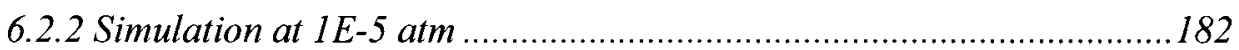

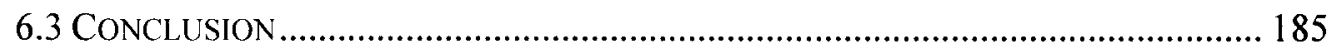

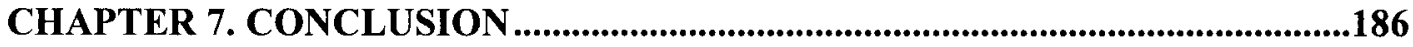

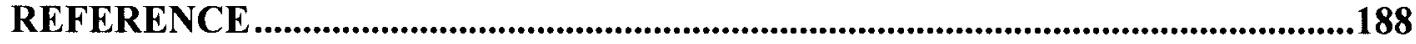

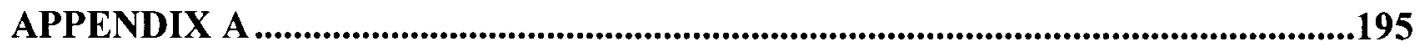

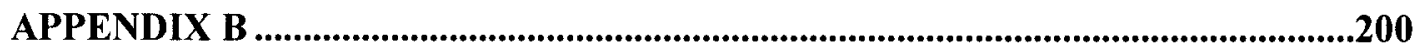

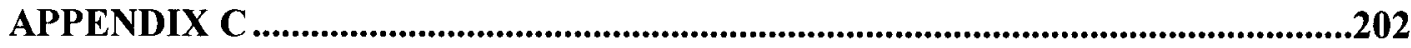

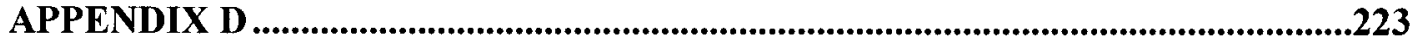

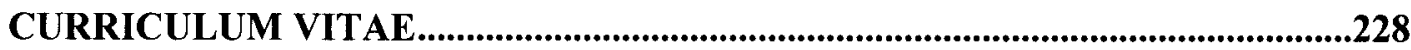

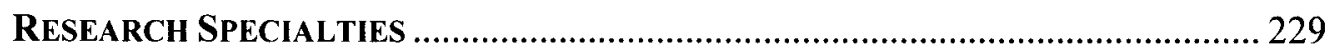

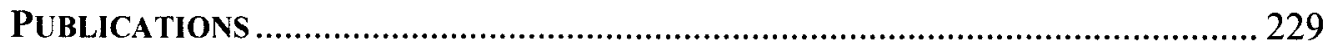




\section{LIST OF FIGURES}

Figure 1. Schematic of microcantilever beam configuration as a gas sensor ...................... 3 Figure 2. SEM images of the T-shaped resonators: (left) top view; (b) tilted view [12] ... 7 Figure 3. T-shaped resonator experiment: (left) SEM image of beam in resonance; (right) experimental setup to monitor beam resonance ( $\mathrm{A}$ is driving electrode)[34] ......... 8 Figure 4. Experimental results for T-shaped resonators detecting gas presence: (left) base voltage as methane gas pressure varies from $0.01-760$ Torr; (right) peak frequency shift observed for several gases at pressure indicated [34] .................................................... 9 Figure 5. Finite element modeling of asymmetric cantilever: (left) coarse 3D mesh (part of beam shown); (right) current density plot in undeflected state[37] 10

Figure 6. Device layout on wafer: (left) wafer view with 72 die (60 useable); (middle) single die with 10 microcantilever beams in an array; (right) single microcantilever beam sensor. 13

Figure 7. Fabrication steps with the region of application indicated schematically (wafer

or die): (left) the original approach; (right) the new approach

Figure8. New equipment used for writing beam pattern: (left) Heidelberg DWL-66FS laser lithography system; (right) SUSS MA6 mask aligner 15 Figure 9. Masks for device fabrication (10 beams when completed): (left) metal lead wire

mask; (middle) bond pad mask; (right) beam pattern mask.

Figure 10. Pattern after beam mask is written and developed. 18 


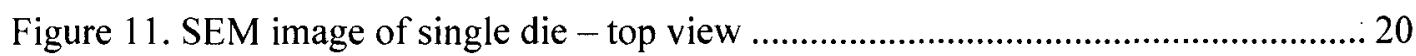

Figure 12. SEM image of single beam - 3D view and close-up view of beam tip.......... 20

Figure 13. The beam of $1.3 \mu \mathrm{m}$ width is measured in SEM ...................................... 22

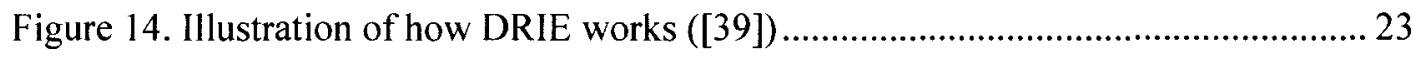

Figure 15. New beam mask to decrease exposed areas ............................................... 25

Figure 16. Schematic of general instrumentation setup.[36] .......................................29

Figure 17. Voltage from lock-in amplifier during driving electrode frequency sweep.... 30

Figure 18. Beam resonating captured with HiSpec ............................................. 30

Figure 19. Beam base resistance during tip displacement cycle for symmetric case: (top)

ANSYS element model; (bottom) normalized resistance change.................................. 32

Figure 20. Beam base resistance during tip displacement cycle for asymmetric case: (top) ANSYS element model; (bottom) normalized resistance change................................ 33

Figure 21. Beam base resistance during tip displacement cycle for asymmetric case as

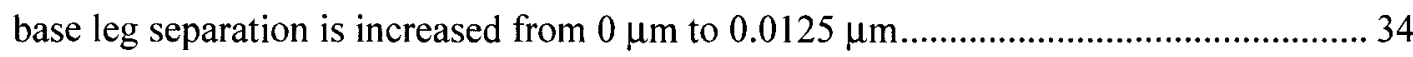

Figure 22. Normalized beam base resistance versus tip displacement: (left) symmetric

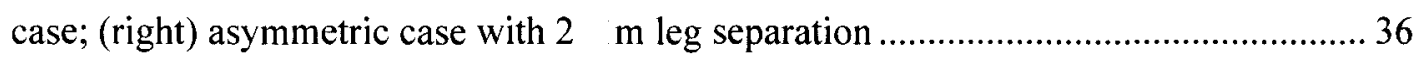

Figure 23. Odd portion $\mathrm{R}_{\mathrm{odd}}(\mathrm{u})$ of normalized beam base resistance versus tip displacement: (left) symmetric case; (right) asymmetric case with $2 \mathrm{~m}$ leg separation 36 Figure 24. Even portion $\mathrm{R}_{\mathrm{even}}(\mathrm{u})$ of normalized beam base resistance versus tip displacement: (left) symmetric case; (right) asymmetric case with $2 \cdots \mathrm{m}$ leg separation 37 Figure 25. Device resistance measurements: (lower left) SEM image of beam with labels A-D; (upper left) DC circuit consisting of resistance between probe station tips and fixed 
resistor; (right) current observed in circuit (should be 0 for all except A-B if oxide is not damaged)

Figure 26. A schematic of the DRIE process: charged parallel plates $(1,4)$ are used with a gas (2) to create ions that bombard (3) the silicon wafer (5)

Figure 27. Schematic of proposed operation with charged handle layer to reduce the likelihood of stiction. 42

Figure 28. Schematic of cantilever beam resonating in a gas: (left) 3D view of beam with tip load $F_{d}$; (right) section view of beam at location $\mathrm{x}$ along beam with local distributed $\operatorname{gas} \operatorname{load} \mathrm{f}_{\mathrm{g}}$.

Figure 29: Schematic diagram of the experiment in which two cylinders move to the left at constant velocity: (top) view looking down the cylinder axes; (bottom) side view with the illuminated layer indicated[45] 50

Figure 30 . Schematic diagram of the experiment in which a cylinder-wall pair move to the left at a constant velocity: (left top) view looking down the cylinder axis; (left bottom) side view with the illuminated layer indicated; particles that were initially in a straight vertical line as the cylinder-plate reaches them to indicate flow regime[45] ..... 51 Figure 31 . The geometry consists of a $2 \mathrm{D}$ tank and two cylinders..............................5 52

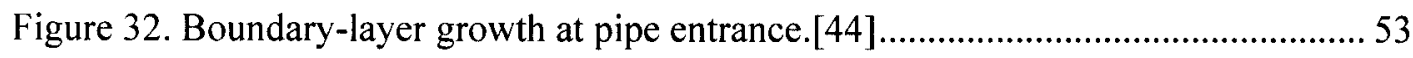

Figure 33. The geometry consists of a 2D tank and one cylinder near bottom. ............... 54

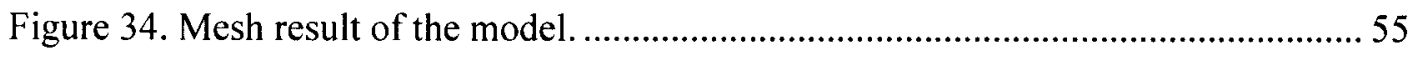

Figure 35. Streamline patterns around two equal circular cylinders: (a) $/ D=1.5$; (b)

$$
/ \mathrm{D}=1.0 ;(\mathrm{c}) \quad / \mathrm{D}=0.5 ;(\mathrm{d}) \quad / \mathrm{D}=0.0
$$


Figure 36. Drag force on each cylinders from CFX simulation compared with the Oseen

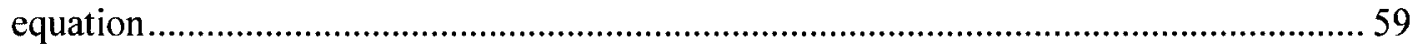

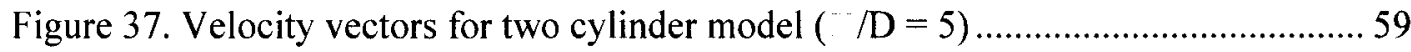

Figure 38 . Stream patterns around a circular cylinder placed near a flat plate: (a) $\_/ D=$

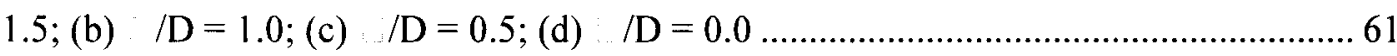

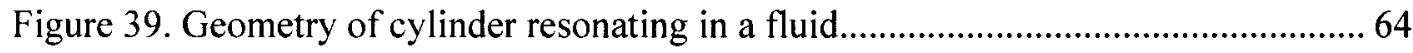

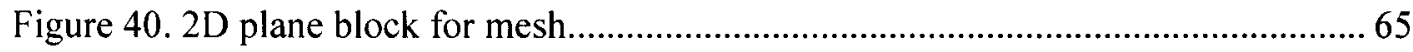

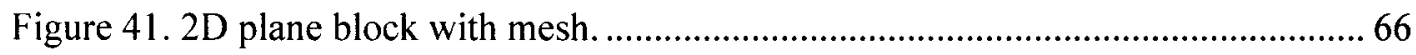

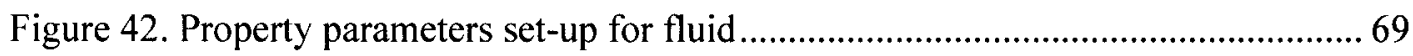

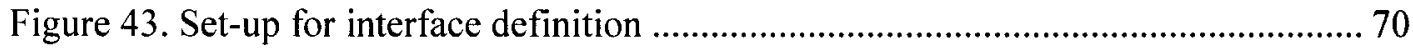

Figure 44 Set-up for tank side-walls definition. (a) Top and bottom; (b) Inlet and outlet.

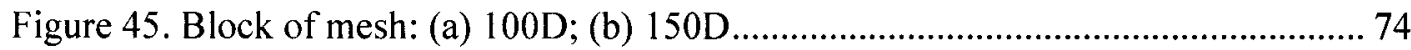

Figure 46. Maximum drag force of each cycle in cylinder surface according to different

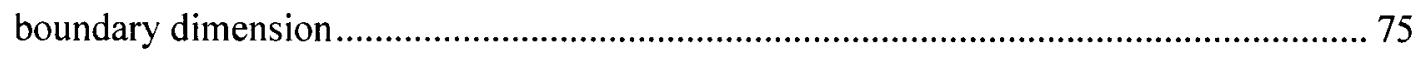

Figure 47. Mesh block of different element number along edge. (a) 40 ; (b) $60 \ldots \ldots \ldots \ldots . . . .76$

Figure 48. Maximum drag force for each cycle in the cylinder surface according to the different element number along the boundary edge. ............................................ 77

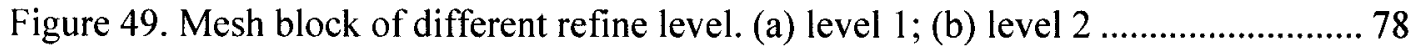
Figure 50. Maximum drag force for each cycle in cylinder surface according to different

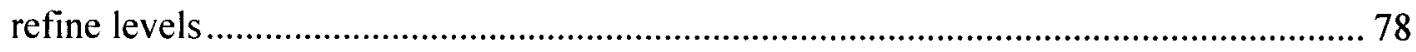
Figure 51. Drag force in the cylinder surface according to the different timestep set-up. 79 
Figure 52. Maximum Drag force for each cycle in the cylinder surface according to the different timesteps in calculation. 80

Figure 53. Drag force in $\mathrm{x}$ direction per unit length versus with time for $S_{0}=0.2 \mu \mathrm{m} \ldots . .81$ Figure 54. Drag force in the cylinder surface according to the different initial resonating distance. 82

Figure 55. Force vs. Time for resonating cylinder from Stoke equation..................... 88

Figure 56. Comparison of results from stoke equation and from CFX........................89

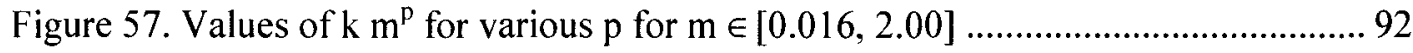

Figure 58. Value of $\mathrm{k}$ using fitting function and using spherical approximation ............. 95

Figure 59. Percent error between fitting function for $\mathrm{k}$ and $\mathrm{k}$ from Stokes' model.......... 95

Figure 60. Value of k' using fitting function and using spherical approximation ............ 96

Figure 61. Percent error between fitting function for k' and k' from Stokes' model ....... 96

Figure 62. Mesh for case of cylinder resonating in middle ...................................... 98

Figure 63. Drag force per unit length vs. time for case of cylinder resonating in middle 99

Figure 64. Mesh for cylinder resonating with floor ............................................. 100

Figure 65. Drag force per unit length vs. time for case of cylinder resonating with floor

Figure 66. Peak of drag force compared between cylinder without floor and with floor 101 Figure 67. Mesh for case of rectangle without floor. 102

Figure 68. Drag force per unit length vs. time for case of rectangle resonating without floor

Figure 69. Peak of drag force compared between rectangle without floor and cylinder without floor... 
Figure 70 Mesh for case of rectangle resonating with floor 105

Figure 71. Drag force per unit length vs. time for case of rectangle with floor 105

Figure 72. Drag force compared between case rectangle with or without floor 106

Figure 73. Drag force vs. frequencies for four cases.

Figure 74. Ratio of drag forces with floor to those without floor.

Figure 75. Rectangle with floor compared with Stokes' equation and cylinder without

floor

Figure 76. Geometry of cylinder resonating near floor with various values of $\delta$. (a) $\delta=0.5$ $\mu \mathrm{m}$, (b) $1 \mu \mathrm{m}$, (c) $4 \mu \mathrm{m}$, (d) $8 \mu \mathrm{m}$, (e) $16 \mu \mathrm{m}$, where $\delta$ is the gap between the bottom of cylinder and the floor.

Figure 77. The maximum drag force versus $\delta$ at $40 \mathrm{kHz}$

Figure 78. Comparison of parallel-plate actuator (left) to current device at driving electrode (right).[51]

Figure 79. Schematic of beam tip 122

Figure 80 . Schematic of beam-electrode pair and vectorial representation of resulting attractive force.

Figure 81. Coupled mechanical-electrostatic model of beam and driving electrode: (top) model of the beam and driving electrode; (middle) voltage contours at $10 \mathrm{~V}$ at driving electrode and beam tip at $0 \mathrm{~V}$; (bottom) associated electrical field. 126

Figure 82. Zoom-in on tip region of associated electrical field 127

Figure 83. Tip deflection vs. driving electrode cycle fraction. (Left) beam at $0 \mathrm{~V}$; (Right) beam at $5 \mathrm{~V}$ 128

Figure 84. Tip deflection vs. $\Delta \mathrm{V}^{2}$. (Left) beam at $0 \mathrm{~V}$; (Right) beam at $5 \mathrm{~V}$. 128 
Figure 85. Beam tip displacement in microns versus voltage applied at the driving electrode when beam is at $0 \mathrm{~V}$.

Figure 86. Driving electrode voltage and beam tip load versus time. 130

Figure 87. $\Delta \mathrm{V}^{2}$ versus time. $(\mathrm{Up}) \mathrm{V}_{\mathrm{E}}=10 \mathrm{~V}, \mathrm{~V}_{\mathrm{B}}=0 \mathrm{~V}, \mathrm{~V}_{0}=0 \mathrm{~V} ;\left(\right.$ Bottom) $\mathrm{V}_{\mathrm{E}}=10 \mathrm{~V} \mathrm{~V}$

$=5 \mathrm{~V}, \mathrm{~V}_{0}=0 \mathrm{~V}$

Figure 88. Variation in $1^{\text {st }}$ natural frequency for asymmetric beam base arrangement with various leg separation and leg length values.

Figure 89. Vertical displacement of cantilever beam changes with time. (Left) 2 cycles;

(Right) 40 cycles 152

Figure 90. Response of a vibration cantilever beam versus frequencies: $F=F_{0} \cdot \sin (\omega \cdot t)$.

Figure 91. Response of a vibration cantilever beam versus frequencies: $F=F_{0} \cdot \cos (\omega \cdot t)$.

Figure 92. Square of ${ }^{\cdots} \mathrm{V}=\left(\mathrm{V}_{\mathrm{e}}-\mathrm{V}_{\mathrm{B}}\right)^{2}$ for $V_{I:}=10, V_{B}=0$. 158

Figure 93. Square of $\mathrm{V}=\left(\mathrm{V}_{\mathrm{e}}-\mathrm{V}_{\mathrm{B}}\right)^{2}$ for $V_{E}=10, V_{B}=5$. 158

Figure 94. Square of $\mathrm{V}=\left(\mathrm{V}_{\mathrm{e}}-\mathrm{V}_{\mathrm{B}}\right)^{2}$ for $V_{E}=10, V_{B}=10$

Figure 95. Beam displacement for $V_{E:}=10, V_{B}=5$ with driving electrode voltage frequency of $1 \mathrm{kHz}$ 160

Figure 96. Beam displacement for static force for $V_{E}=10, V_{B}=5$. 161

Figure 97. Beam displacement for $V_{E}=10, V_{B}=5$ with driving electrode voltage frequency of $\omega_{n} / 3$ 161 
Figure 98. Beam displacement for $V_{E}=10, V_{B}=5$ with driving electrode voltage frequency of $\omega_{\mathrm{n}}$ 162

Figure 99. Beam displacement for $V_{E}=10, V_{B}=5$ with driving electrode voltage

frequency of $\omega_{n} / 2$. 162

Figure 100. Peak of displacement versus frequency for $u$ : frequency swept (a) from 40-50 $\mathrm{kHz}$; (b) from $80-100 \mathrm{kHz}$; (c) from $20-120 \mathrm{kHz}$. 164

Figure 101. Effective viscosity me for nitrogen at $300^{\circ} \mathrm{K}$ from simulation and compared to several models; the solid line corresponds to Eq. (127) with a $=2$. [58] 168

Figure 102. Peak amplitude of displacement vs. frequency with pressure as parameter. 170 Figure 103. Response curve for different pressures from Yang's experiment data. [42]

Figure 104. Peak displacement during a cycle as a function of driving electrode frequency (normalized by $\mathrm{nl}=92.378 \mathrm{kHz}$ ) for $\mathrm{V}_{\mathrm{E}}=10$ and $\mathrm{V}_{\mathrm{B}}=5$. 174

Figure 105. Electrostatic force (in N ) versus time for $f=f_{n 1}=92.378 \mathrm{kHz} \ldots \ldots \ldots \ldots \ldots . . .175$

Figure 106. Displacement of beam tip (in $\mathrm{m}$ ) versus time for $\mathrm{f}=\mathrm{f}_{\mathrm{n} 1}=92.378 \mathrm{kHz} . \ldots .176$ Figure 107. Change of normalized symmetric base resistance versus time for $f=f_{n 1}=$ $92.378 \mathrm{kHz}$ 176

Figure 108. Change of normalized asymmetric beam base resistance versus time for $\mathrm{f}=$ $\mathrm{f}_{\mathrm{nl}}=92.378 \mathrm{kHz}$ 177

Figure 109. Voltage change measured by lock-in amplifier for symmetric beam base versus time for $f=f_{n 1}=92.378 \mathrm{kHz}$. 177

Figure 110 . Voltage change measured by lock-in amplifier for asymmetric beam base versus time for $f=f_{n l}=92.378 \mathrm{kHz}$. 


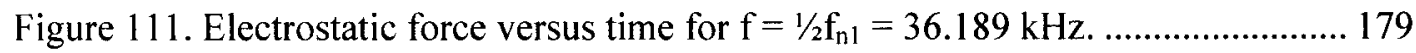

Figure 112. Displacement of beam tip versus time for $f=1 / 2 f_{n 1}=36.189 \mathrm{kHz} \ldots \ldots \ldots \ldots \ldots .179$

Figure 113. Change of normalized symmetric base resistance versus time for $f=1 / 2 f_{n 1}=$ $46.189 \mathrm{kHz}$. 180

Figure 114. Change of normalized asymmetric base resistance versus time for $f=1 / 2 f_{n 1}=$ $46.189 \mathrm{kHz}$. 180

Figure 115. Voltage change measured by lock-in amplifier for symmetric beam base versus time for $\mathrm{f}=1 / 2 \mathrm{f}_{\mathrm{n} 1}=46.189 \mathrm{kHz}$

Figure 116. Voltage change measured by lock-in amplifier for asymmetric beam base versus time for $f=1 / 2 f_{n 1}=46.189 \mathrm{kHz}$ 181

Figure 117. Displacement of beam tip versus time: (left) $f=f_{n 1}$; (right) $f=1 / 2 f_{n 1}$ 182

Figure 118. Change of normalized beam base resistance for symmetric base versus time:

(left) $f=f_{n l} ;$ (right) $f=1 / 2 f_{n 1}$ 183

Figure 119. Change of normalized beam base resistance for asymmetric base versus time: (left) $f=f_{n l} ;$ (right) $f=1 / 2 f_{n 1}$ 183

Figure 120. Voltage into lock-in amplifier for symmetric base versus time: (left) $f=f_{n 1}$; (right) $f=1 / 2 f_{n 1}$ 184

Figure 121. Voltage into lock-in amplifier for symmetric base versus time: (left) $f=f_{n 1}$;

(right) $f=1 / 2 f_{n 1}$ 184 


\section{LIST OF TABLES}

Table 1. Fabrication time (hrs) for original and improved fabrication methods. ............. 21

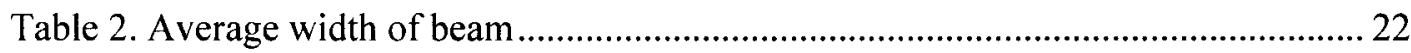

Table 3. Parameters for fluid used in experiment.3.2.3 Setup in CFX - Cylinder with wall experiment

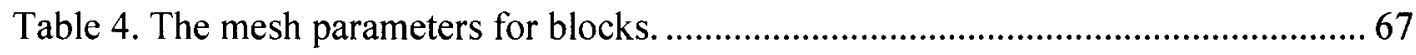

Table 5. Nodes number and element length along the dialog of square ....................... 74

Table 6. Summary of mesh element number and calculation time .................................. 77

Table 7. Numerical calculation of $k$ and $k$ ' for different value of $m$............................... 85

Table 8. Parameters used in calculation of force acting at the cylinder. ....................... 87

Table 9. Comparison of peak results from stoke equation and from CFX ..................... 88

Table 10. Fitting model coefficients for Stokes' cylinder model ..................................99

Table 11. Peak of drag force compared between cylinder without floor and with floor 101

Table 12. Peak of drag force compared between rectangle without floor and cylinder

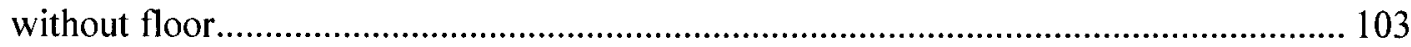

Table 13. Drag force compared between case rectangle with or without floor .............. 106

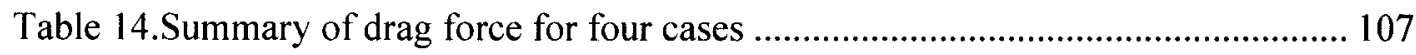

Table 15. Setup of time-steps for different frequencies....................................... 108

Table 16. Peak drag force per unit length $(\mathrm{N} / \mathrm{m})$ for four cases and different frequencies

Table 17. Ratio of drag forces of rectangle and cylinder.......................................... 112 
Table 18. Ratio of drag forces with floor to those without floor versus frequency ........ 113

Table 19. Compare Stokes equation and cylinder without floor ................................ 114

Table 20. Compare the Stokes equation with rectangle with floor ............................... 114

Table 21. Compare the cylinder without floor and rectangle with floor ...................... 115

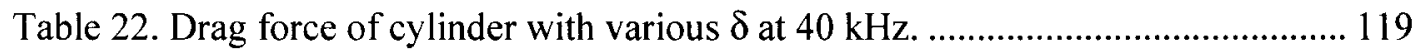

Table 23. Displacement factors (in $\mathrm{m}$ ) versus different frequency......................... 157

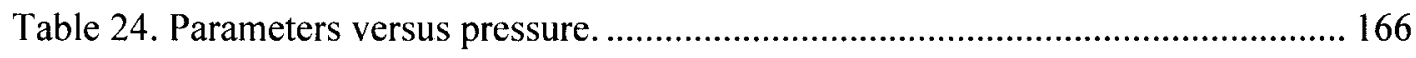

Table 25. Damping parameters versus pressure after using effective viscosity formula

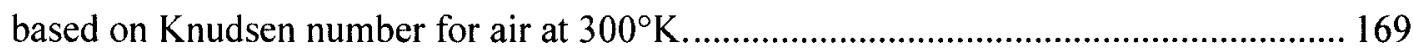




\section{CHAPTER 1. INTRODUCTION}

Piezoresistive microcantilever beams have been demonstrated in previous work by the researchers at the University of Louisville to be capable of sensing the presence of surrounding gas by changes in the resonance behavior of the beam. Several device improvements and modifications have been pursued over the past several years including alternate fabrication methods and asymmetric base configuration to improve sensitivity. However, a number of challenges remain to reach the point where devices can be reliably fabricated and operated in a consistent and repeatable manner in a gas environment.

The ultimate goal of this device development is to create an integrated highsensitivity system for gas detection, with an intended application towards the early positive identification of terrorist threats and hazardous materials. The work described in this dissertation is intended to overcome a number of the remaining difficulties; these include improvements to speed fabrication, fluid flow modeling to better address the drag forces which act on the oscillating beam, simulation of electrostatic forces acting on the beam and its associated vibration response. The purpose of these steps is to advance the state of the art in the area of microcantilevers beams used for gas sensing and detection. As such, they will represent an important step in realizing the potential for these devices for future applications. 


\section{I.I Background Review}

In recent years, rapid development in micro/nano actuation-based sensor technology has become widely used for gas detection and biochemical analysis. [1-2] Devices based on resonating cantilever beams represent one popular type of sensor due to its simple geometry, fast response times and high sensitivity.[3] Cantilever beams operating in a gas or fluid environment experience a resonance frequency shift and change in vibration amplitude around resonant frequency due to the damping effect.[4] Similarly, coated cantilever beams that selectively attach to various compounds will experience a resonance frequency shift due to the added mass.[5]

In order to assess resonance, a method for monitoring the vibration amplitude is required. One common approach uses laser-based optical methods for this measurement. [4, 6-9] Another approach is to use piezoresistive methods to detect the beam vibration behavior.[10-12] Piezoresistance-based microcantilever sensor are becoming more popular for they are convenient to calibrate, readily deployable into integrated electromechanical system and do not require external detection devices for measuring surface stress as a result of the binding of chemical and biological species.[1314] Although this method avoids complicated optical equipment, it also introduces other challenges due to the fact that it relies on understanding the stress state in the monitored structure requires knowledge of the piezoresistive material properties, and the results can be sensitive to temperature changes in the beam.

As a gas sensor, the microncantilever beam system can be considered as 3 components as shown in Figure 1. The two inputs are the gas environment surrounding the beam and the electrical signal at the driving electrode that induces small periodic 
forces in the region around the beam tip due to capacitive effects. The microcantilever beam and its base form the sensor. At certain driving electrode frequencies, the beam enters resonance; the magnitude and frequency of vibration are affected by the surrounding gas. The base legs that support the beam have induced periodic stresses due to the beam motion. The output of the sensor comes from the piezoresistive effect; base leg stresses cause periodic resistance change that can be measured via a DC circuit across them. The magnitude of this signal therefore provides a measure of the beam motion. Resonance can also be assessed using optical methods as will be shown in several images below; however, the long-term goal of these sensors is to avoid the need for complex microscopy or other equipment required for optical approaches. The observed beam motion can then be combined with suitable beam vibration and fluid mechanics models to estimate the damping effect of the underlying gas.

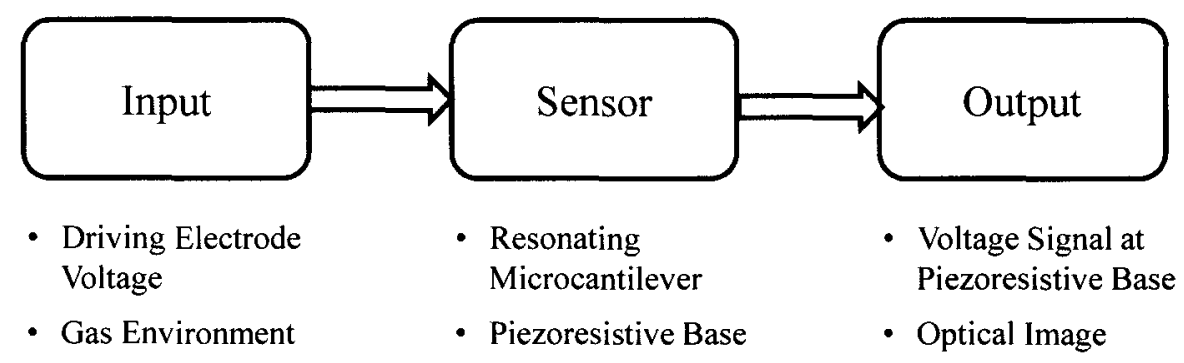

Figure 1. Schematic of microcantilever beam configuration as a gas sensor

Past and current efforts have focused on improving various aspects the system above. One of these is the fabrication method used in the creation of the device. For piezoresistive microcantilever beam devices, common fabrication approaches include photolithography and combinations of dry or wet etching.[2] As the beam size reached the order of $0.5-1 \mu \mathrm{m}$ width, limitations of photolithography equipment initially 
required the use of time-consuming methods such as e-beam lithography to create the necessary small feature sizes.[12] Recent work by the author has focused on using newer equipment to fabricate the sensors using alternate fabrication methods; these efforts have been achieved in reducing the fabrication time and increasing the number of operational devices achieved per wafer. However, this new method also leads some new problems that do not exist in old approaches. These new problems include variation of beam width crossing the whole wafer and leakage between the device and the handling layer. These issues need to be further investigated.

Another area of interest is in improved device sensitivity. The original configuration at UofL, utilizing micro cantilever beams supported by symmetric piezoresistive base legs, has been demonstrated in previous research to be capable of sensing the presence of surrounding gas. Unfortunately, the symmetric configuration leads to very low sensitivity, with base leg resistance changes on the order of $10^{-6}$ times the unstressed resistance value. Research at UofL has utilized an asymmetric base arrangement in the sensor design; this has greatly improved device sensitivity.[15-16] Other researchers have pursued size reductions towards the nanoscale to increase sensitivity of beam sensors. [17-18] Similarly, other attempts have been made to improve the sensitivity and resolution of piezoresistive microcantilevers by reducing the beam thickness.[19] Improved sensitivity offers the potential for identifying small changes in gas properties, thereby increasing the likelihood of detection of an undesirable component.

Testing of the sensors offers another challenge as physical dimensions of devices are on the scale of microns and mechanical resonant frequencies are typically in the range 
of $10^{4}-10^{5} \mathrm{~Hz}$. As such, traditional vibration excitation techniques, such as impact hammer and shaker tables cannot be readily used to induce vibration in order to characterize microstructural response. Electrostatic excitation is one of the general excitation methods for microscale devices; in the current sensor design, electrostatic excitation forces are exerted by a driving electrode separated from the beam tip by a small gap. The disadvantages of this method are that electrostatic forces are nonlinear functions of structural motions and that there is coupling between electrostatic and structural fields.[20] In addition to the challenges of inducing vibration, microscale devices are quite sensitive to a host of effects, such as the viscosity of any surrounding gas, that could often be neglected when dealing with traditional macrostructures. As such, the dynamic characteristics of a microstructure depend strongly on surface and interface effects compared to the volume or bulk effects of the microstructure itself.[21]

The purpose of the sensor as described is to ultimately detect resonance changes caused by a surrounding fluid or gas. To accomplish this, suitable models must be identified that can relate the observed changes to appropriate media properties such as viscosity and density. Blom et al. in 1992 utilized a model based on spheres vibrating in an infinite media to assess damping effects for a cantilever beam; this model indicated that the shift in resonance frequency of a microcantilever was a function of the molar mass and gas pressure.[22] This finding provided an avenue to assess the molar mass of the gas could be obtained by measuring the resonance frequency shift.[12, 23] Of course, a cantilever beam of rectangular (or other) cross-section does not really resemble a series of spheres. As such, various researchers have created a variety of other fluid-structure models to assess the damping effect; these have included both inviscid and viscous fluid 
models, cylindrical and thin blade geometries, and incorporation of the media flow rate in this assessment.[24-33]

While each of these approaches may be useful, none of them consider the configuration used in the current sensor design - namely that the microcantilever beam is a plane that resides approximately $2 \mu \mathrm{m}$ above a fixed, flat surface (the handle layer of the SOI wafer). The approaches above consider resonating structure in an infinite fluid domain model, while the actual structure resembles as semi-infinite fluid domain problem. If the floor plays a significant role in terms of the viscous forces acting on the resonating beam, this effect will not be captured using a model such as that developed by Blom et al. The approach considered in this dissertation utilizes a CFD model to estimate the effect of area-cross of cantilever beam (comparing cylinder to rectangular) and effect of wall due to floor presents. This model is useful in understanding the hydrodynamic drag on a microcantilever moving through a fluid at a constant speed [29]; it is also matched drag force with the one from Stokes' oscillating cylinder model. The effects of area-cross and floor can be expressed in formula after comparing the rectangular beam with floor with Stokes' oscillating cylinder beam. This effects then acts as a factor in calculation of coefficients of drag force.

\subsection{Past Research at UofL}

Research work by Yang Xu during her doctoral studies in Mechanical Engineering at the University of Louisville demonstrated methods to create narrow, piezoresistive microcantilever beams; these beams were demonstrated to be capable of detecting the presence of various gases via changes in the resonance characteristics of the beam. These structures were all symmetric about the beam midplane.[12, 23] One style of 
microcantilever considered in Dr. Xu's work was the T-shaped resonator as shown in Figure 2 . The beam $(128 \mu \mathrm{m}$ long $\times 1.1 \mu \mathrm{m}$ wide $\times 3 \mu \mathrm{m}$ deep $)$ is attached to a shorter base $(9 \mu \mathrm{m}$ wide) that connects two electrodes. Near the end of the beam, a driving electrode is used to actuate the beam; by applying an AC voltage at the driving electrode, the beam will experience periodic electrostatic forces. It will enter a state of resonance when the $\mathrm{AC}$ frequency is equal to certain specific values that depend upon the geometry and material properties of system.
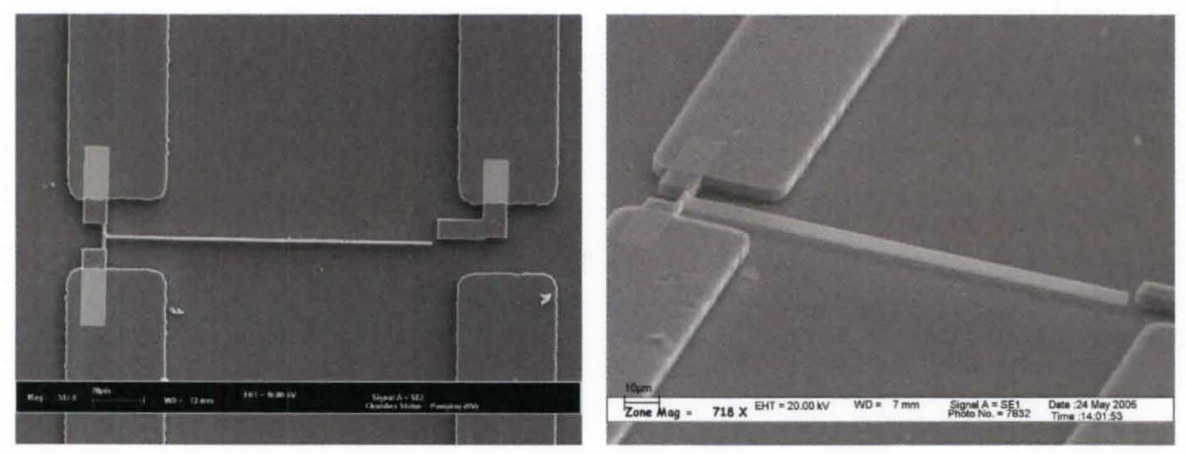

Figure 2. SEM images of the T-shaped resonators: (left) top view; (b) tilted view [12]

The material used (boron-doped silicon) was piezoresistive, meaning that a state of stress changes the resistance of the material. Deflections of the beam lead to stresses in the base and, hence, associated resistance changes in the base. When the AC voltage at the driving electrode causes the beam to vibrate at frequency $f$, the beam base resistance changes occur at frequency $2 f$ since motion in either direction causes an identical change in resistance due to symmetry. The magnitude of this resistance change can be detected by passing a constant DC voltage across a circuit consisting of the beam base (varying resistance) and a fixed resistor; a lock-in amplifier seeks the value of the signal at frequency $2 f$ which can then be correlated to beam deflection. 
An example of a T-shaped beam in resonance and the associated experimental setup are shown in Figure 3; note that the atmosphere in which the beam is located (probe station chamber) can be varied from full vacuum to a desired pressure using various gases. An example of beam deflection amplitude (as observed by base resistance changes) versus frequency is shown in Figure 4 for a $\mathrm{T}$-shaped resonator in methane gas at various pressures; this shows both the peak frequency shift as well as broadening of the response curve as the pressure (and hence viscous damping on the beam) increases. Figure 4 also shows the peak frequency shift relative to the vacuum case $\left(f / f_{0}\right)$ and demonstrates a different result for each gas considered; using the model by Blom et al [22], this shift was then shown to be related to the gas molar mass.
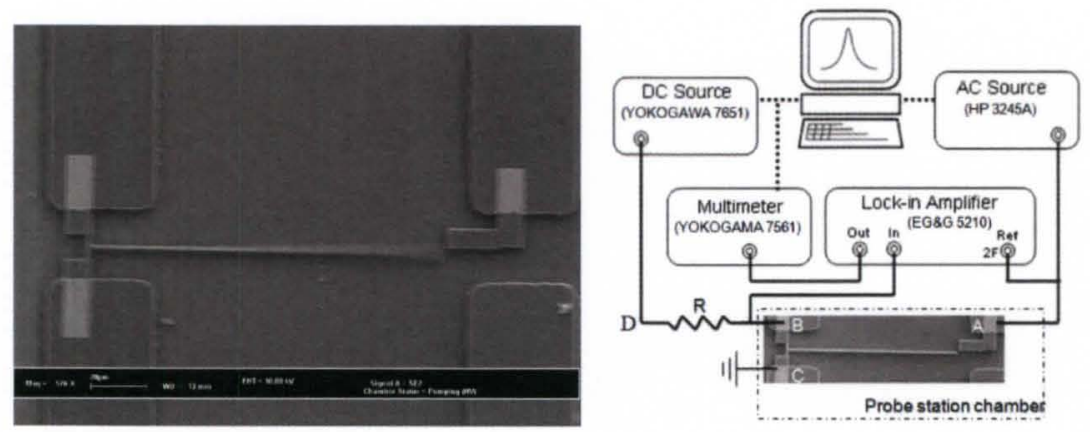

Figure 3. T-shaped resonator experiment: (left) SEM image of beam in resonance;

(right) experimental setup to monitor beam resonance (A is driving electrode)[34] 

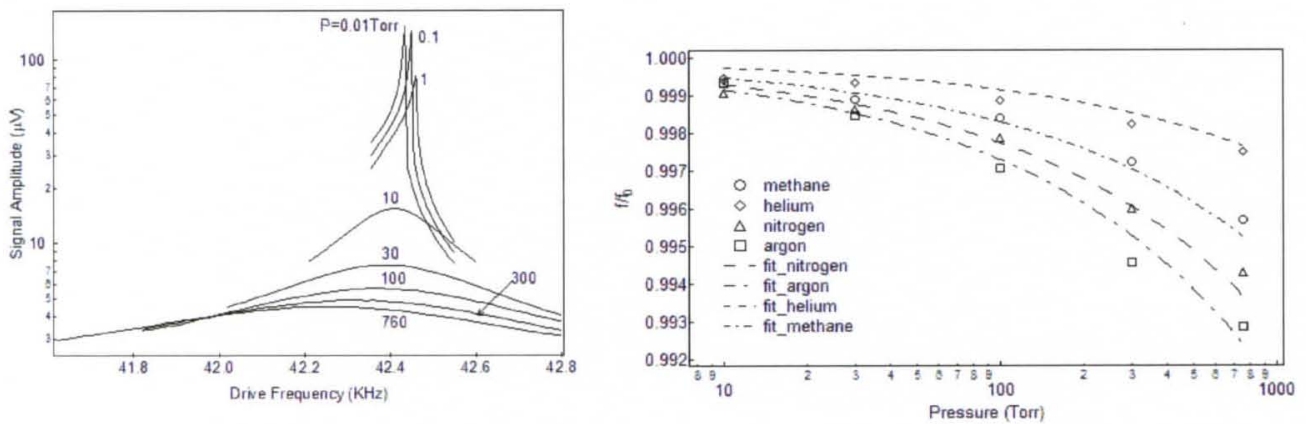

Figure 4. Experimental results for T-shaped resonators detecting gas presence:

(left) base voltage as methane gas pressure varies from $0.01-760$ Torr;

(right) peak frequency shift observed for several gases at pressure indicated [34]

While the results above are promising, there were several challenges that became apparent during this project. First, the piezoresistive changes the beam base were relatively low, meaning that beam resonance lead to relative resistance changes on the order of 1-10 parts per million (not far above the system noise level). Second, the fabrication method was complex and time consuming, involving many processing steps across multiple equipment pieces to create a device.

Base resistance sensitivity has been improved using an asymmetric (or staggered leg) base arrangement. In the original device, the beam base is in a state of bending and many of the resistance changes cancel out due to the tension/compression nature of the stress state. The alternate configuration reacts the base loads associated with beam deflection primarily via a force couple from each leg; this leads to both legs having a similar state of stress and therefore a much larger resistance change compared to the original device. This effect was studied using numerical methods to validate the concept; an example of a 3D finite element model and its associated current density plot used to 
determine base resistance is shown in Figure 5.[35] These numerical studies indicated relative resistance changes on the order of 1\%, a 3-4 magnitude order increase compared to the original design. Limited experimental studies using this configuration have shown base resistance changes that are 15-200 times greater than the original design; this work was performed by Patrick Fletcher, an M.Eng. student in Mechanical Engineering who graduated in 2008.[16, 36]
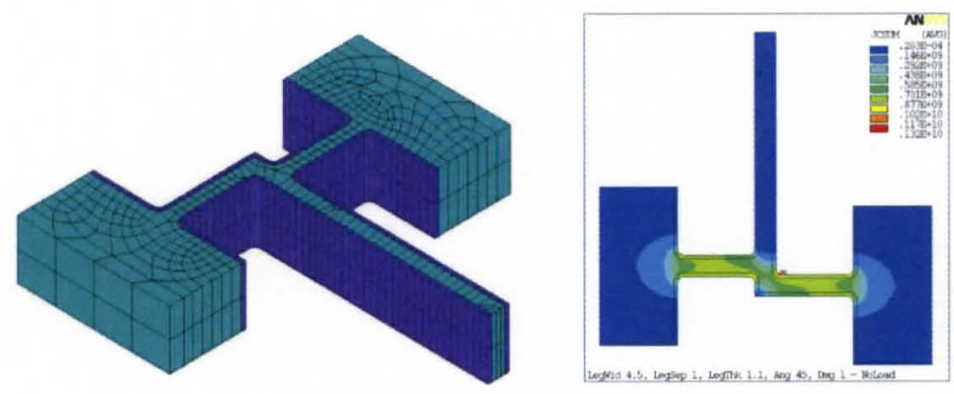

Figure 5. Finite element modeling of asymmetric cantilever: (left) coarse 3D mesh (part of beam shown); (right) current density plot in undeflected state[37]

\subsection{Overview of Dissertation}

As described above, the work of $\mathrm{Xu}$ and Fletcher provides a strong basis from which to further develop microcantilever beam systems for gas detection. However, these studies also indicated several of the limitations already described, including timeconsuming fabrication, low device sensitivity and reliance on fluid models using simplifications that may not be accurate for the configuration used.

In order to address these issues, the author began work on the topic of improvements of microcantilever beams for gas detection and sensing in 2008. The goal at the outset was to extend the work that was done by previous studies towards the long- 
term goal of developing gas detection systems based on arrays of microcantilever beams operating simultaneously and in real-time in a field setting.

To move towards this goal, the author began the series of investigations intended to advance the state of the art in this area. The list below is a brief statement of each item of interest:

Improved Fabrication Approach (Chapter 2)

Modeling of Gas Damping Effects (Chapter 3)

Modeling Electrostatic Forces Driving Resonance (Chapter 4)

Beam Vibration Response (Chapter 5)

Simulation of Voltage Signal at Lock-in Amplifier Simulation (Chapter 6)

Chapter 2 presents fabrication methods developed in this research work as well as measurements of beam dimensions and device yield. Chapter 3 utilizes a combination of analytical models with computational fluid dynamic simulation to assess gas damping loads on the beam. Chapter 4 uses coupled-field finite element simulation to address electrostatic forces; these are then used to predict the beam load under a sinusoidal driving electrode voltage with constant beam voltage. Chapter 5 uses classical vibration models to assess the microcantilevers response during resonance to predict the beam response in operation. Chapter 6 simulate the voltage signal in lock-in amplifier using derived relationship between electrostatic force and displacement of beam tip, the displacement of beam tip and the change resistance of beam base, the change of resistance of beam base and voltage of lock-in amplifier. This signal calculation helps to understand the captured signal responding to the behavior of the cantilever beam. 


\section{CHAPTER 2. DEVICE FABRICATION AND OPERATION}

In this section, the focus is on dissertation topics related to device fabrication and operation in vacuum. This work achieved the goal of improving the speed the fabrication; however, several challenges remain that are also highlighted.

\subsection{Original Fabrication Method}

In both the original and new methods, a commercial $100 \mathrm{~mm}$ (4 in) silicon-oninsulator (SOI) wafer, obtained from Ultrasil Corporation, is utilized for device fabrication. This wafer consists of three layers: a boron-doped silicon device layer (2 microns thick); a buried silicon oxide layer ( 3 microns thick); and a silicon handle layer (500 microns thick). As shown in Figure 6, one wafer could be used to fabricate 60 working dies (12 unusable due to straddling the wafer edge), with each die containing 10 separate microcantilever beam sensors. 


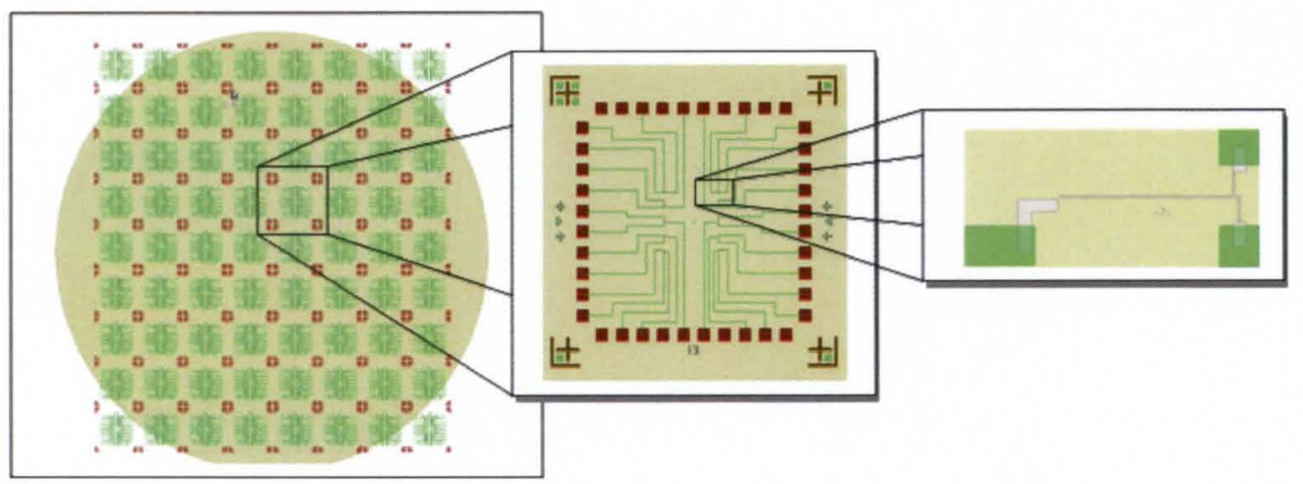

Figure 6. Device layout on wafer: (left) wafer view with 72 die (60 useable); (middle) single die with 10 microcantilever beams in an array; (right) single microcantilever beam sensor.

Steps 1-3 in the original approach (shown in Figure 7(a)) and the new approach (shown in Figure 7(b)) are very similar; these create the gold leads and bonding pads used in the electrical circuitry and are completed using standard photolithography methods. For Step 4 of the original method, two PMMA layers are spun onto the wafer. The pattern for each beam is then written onto the PMMA layers using e-beam lithography; this approach was required since the photolithography mask system available at that time was not capable of feature sizes on the order of 1 micron. Due to the low magnification available in the e-beam lithography system, each microcantilever beam pattern had to be aligned and exposed separately. Therefore, the same manual procedure had to be repeated 10 times for each device on a die, leading to a total of 600 identical operations for an entire wafer. It takes approximately 2 hours to write the beam pattern on a single die or approximately 120 hours for an entire wafer. After the PMMA 
pattern is developed, a $5 \mathrm{~nm}$ thick iron layer is sputtered onto the die to serve as a DRIE mask; an acetone liftoff leaves behind the iron mask with the proper beam pattern.

At this point, the wafer is cut into squares using a dicing saw. Step 5 uses deep reactive ion etching (DRIE) to create the beams by removing any unmasked silicon down to the silicon oxide layer; this method is chosen because it is capable of forming high aspect ratio vertical sidewalls in silicon without etching the silicon-dioxide layer. Buffered oxide etching followed by critical point drying eliminates the silicon oxide below the beam and leads to the final free-standing beam configuration; note that the iron layer remains once fabrication is complete. Detailed description of this fabrication approach is provided elsewhere.[12,23] While this process was successful in fabricating both symmetric and asymmetric beam configurations, it was quite time-consuming and complex to use.

(1)

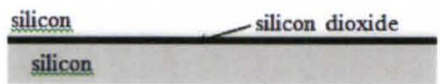

(2)

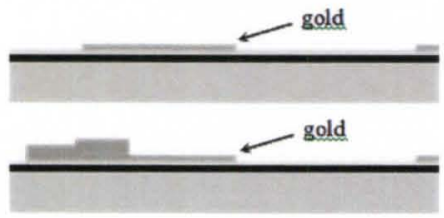

(4)

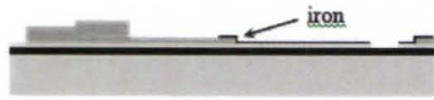

(5)

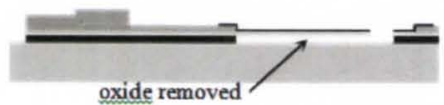

(1)

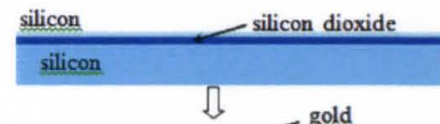

(2)

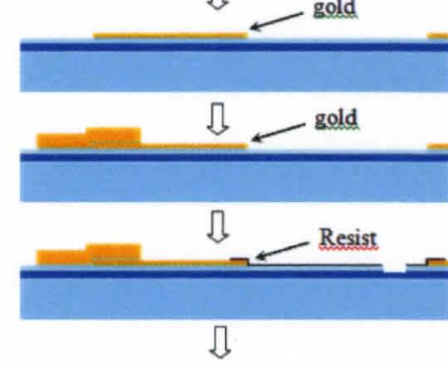

(5)

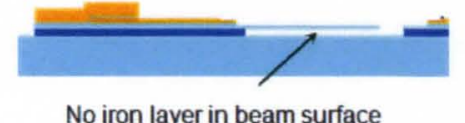

No iron layer in beam surface
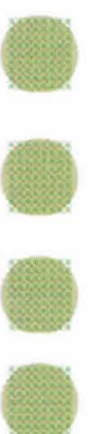

Figure 7. Fabrication steps with the region of application indicated schematically (wafer or die): (left) the original approach; (right) the new approach 


\subsection{Improved Fabrication Method-Details}

The improved fabrication method described in this paper is based upon the use of traditional photolithography methods to eliminate the need for e-beam lithography and iron layer masks; this approach was suggested by Mark Crain of the UofL MicroNanotechnology Center. Two pieces of equipment permitted this change. The first is a Heidelberg DWL-66FS laser lithography system (Figure8(a)); this is used to generate the various masks used and is capable of a writing structure sizes down to 0.6 microns (below the $0.9-1.1$ micron beam widths used in the microcantilevers beams). The second is the SUSS MA6 mask aligner system (Figure8(b)); this permits vacuum contact between the mask and the substrate and permits the writing of submicron patterns. Finally, the use of Shipley 1805 photoresist was an improvement over the original process; this material proved sufficient to serve as the DRIE mask and thus eliminated the need for the iron mask layer.
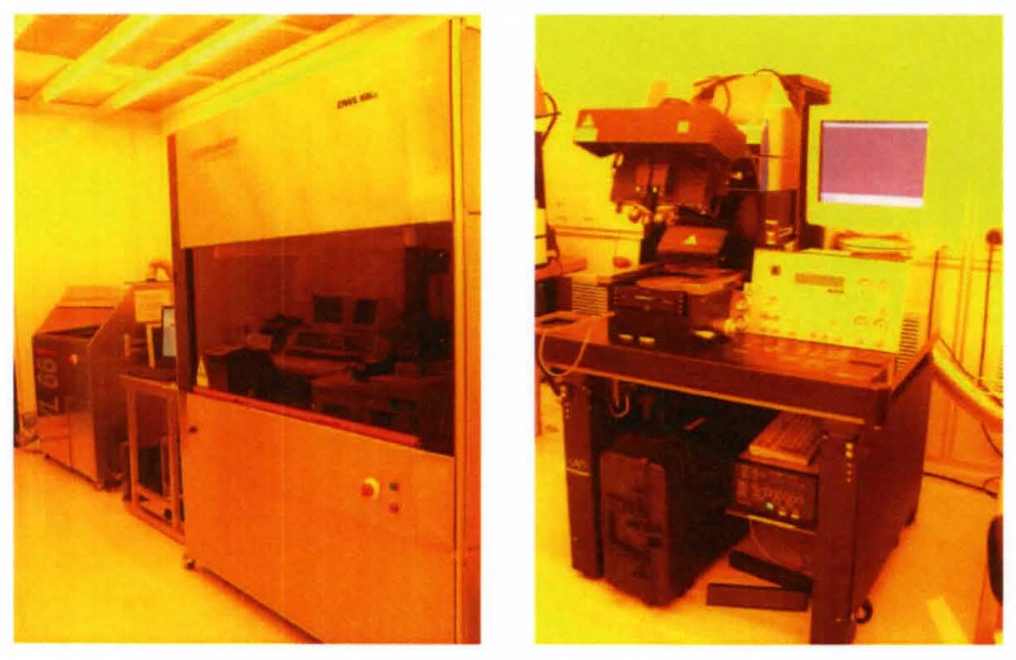

Figure8. New equipment used for writing beam pattern: (left) Heidelberg DWL-66FS laser lithography system; (right) SUSS MA6 mask aligner. 
The new equipment and photoresist simplifies the overall fabrication process to three lithography steps. The three masks (metal lead mask, bond pad mask, beam pattern mask) used in the optical lithography process are shown in Figure 9. The first step creates the electrical leads wires (step 2 in Figure 7). Shipley 1827 positive photoresist is applied to the silicon surface using a wafer spinner, with a spread speed of $500 \mathrm{rpm}$ for 0.2 seconds and a spin speed of $3000 \mathrm{rpm}$ for 10 seconds. The wafer is then soft baked on a hotplate for 1 minute at $115^{\circ} \mathrm{C}$ to remove excess solvent from the positive resist. Next, the substrate is exposed to UV light for 15 seconds using the SUSS MA6 with the mask shown in Figure 9(a). This pattern is then reversed in a YES oven for 45 minutes at $90^{\circ} \mathrm{C}$ followed by a floor exposure performed in the SUSS MA6 for 22 seconds. The resulting pattern was developed in MF 319 for 1 minute with lateral agitation, rinsed with DI water, and then dried. An adhesion layer of $10 \mathrm{~nm}$ thick chromium was RF sputtered onto the substrate at $350 \mathrm{~W}$ for 27 seconds; this was followed by a $35 \mathrm{~nm}$ thick layer of gold that was DC sputtered onto the substrate at $120 \mathrm{~W}$ for 30 seconds. After sputtering, a liftoff process was performed by submerging the wafer in a recirculation acetone bath for 20 minutes to remove the excess gold and chromium, leaving the desired electrode lead pattern on the substrate. The use of the YES oven reduces the time required for the liftoff procedure compared to the original method which did not perform the image reversal step. The identical procedure and parameters was used to create the bonding pads (step 3 in Figure 7) except that the mask used is shown in Figure 9(b) and the DC sputtering time was increased to 103 seconds to increase the thickness of the gold pads to provide a suitable surface for bonding to external electrical connections. 

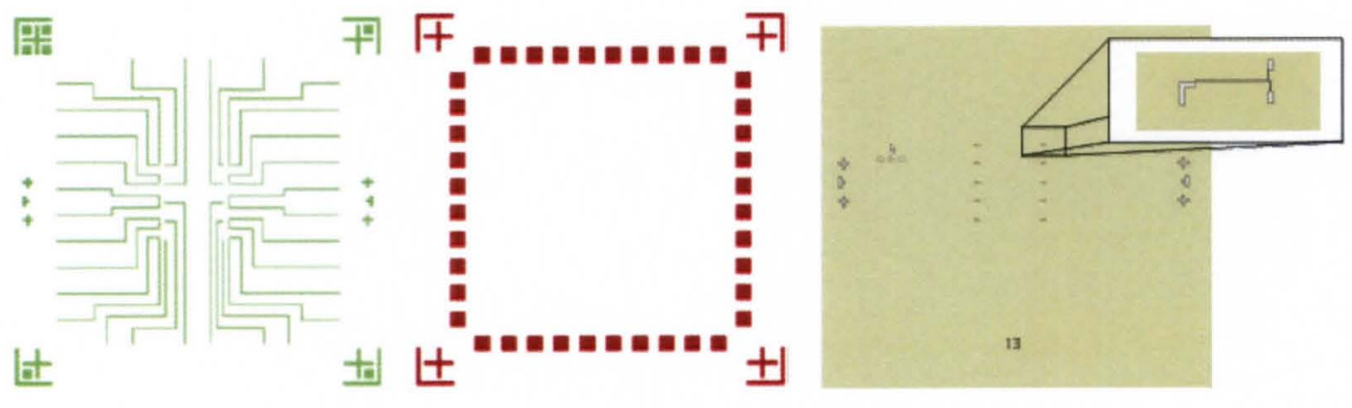

Figure 9. Masks for device fabrication (10 beams when completed): (left) metal lead wire mask; (middle) bond pad mask; (right) beam pattern mask.

The key improvement to the fabrication procedure is in the creation of the beam pattern mask used for the anisotropic deep reactive ion etching (DRIE) step to create the beams (step 4 in Figure 7). First, the wafer with gold leads and bonding pads was baked for 5 minutes at $115^{\circ} \mathrm{C}$ on a hotplate to remove excess moisture and promote photoresist adhesion. Shipley 1805 photoresist was then applied to the silicon surface using a wafer spinner at a spread speed of $500 \mathrm{rpm}$ for 0.2 seconds and a spin speed of $3000 \mathrm{rpm}$ for 10 seconds; this resulted in a thickness of approximately $200 \mathrm{~nm}$. The wafer is then soft baked at $115^{\circ} \mathrm{C}$ for 1 minute to remove excess solvent from the positive resist. The wafer is then exposed to UV light for 5 seconds using beam pattern mask (Figure 9(c)) using the SUSS MA6. The resulting pattern was developed in MF 319 for 30 seconds and rinsed in DI water; agitation is not used during development and the rinse is done very gently to avoid detaching the mask from the wafer surface. Once developed, the beam pattern is checked using an optical microscope to ensure that the pattern is clear and neat; Figure 10 shows a typical pattern after completion of the beam mask step. Note there are several important distinctions between this approach and the original fabrication: 1) timeconsuming e-beam lithography is not required; 2) the need for an iron mask layer is 
eliminated; and 3) the process can be performed on an entire wafer rather than on a single die.

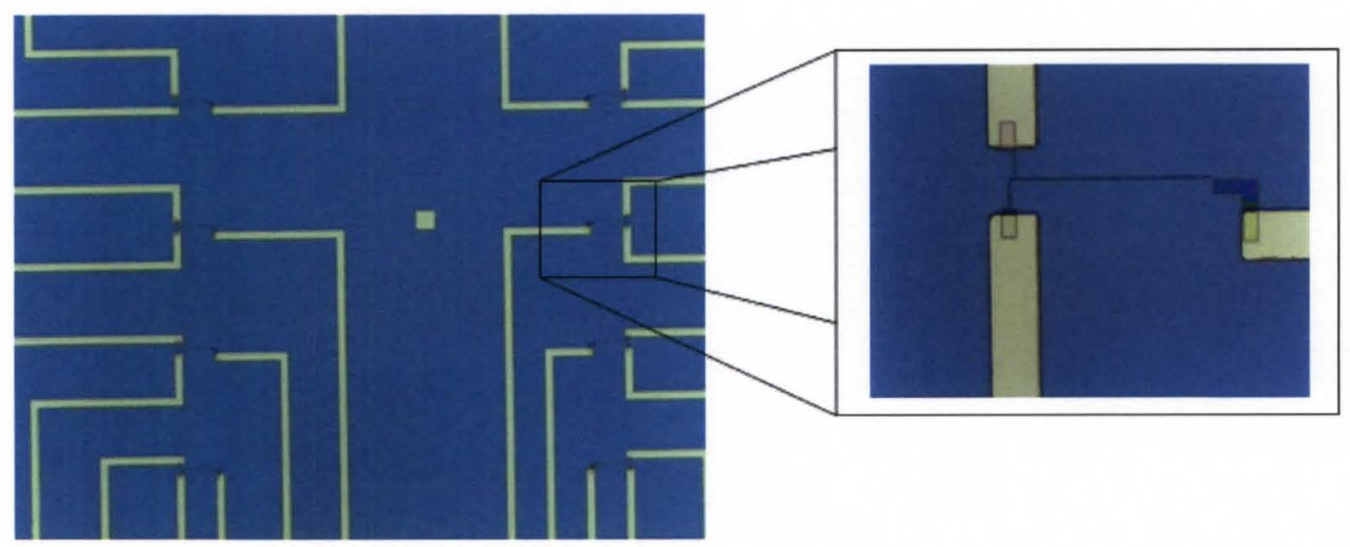

Figure 10. Pattern after beam mask is written and developed.

DRIE is then performed to etch the silicon device layer of the wafer using a base pressure of $0.2 \mathrm{mT}$ Torr and a process pressure of $10 \mathrm{mT}$ Torr at room temperature. The DRIE process was performed in multiple short-time steps to prevent over-etching and damaging the cantilever beams, with optical monitoring of the wafer used to assess when the silicon oxide layer is reached via an observed color change. The average etch rate is $\sim 0.17 \mu \mathrm{m} / \mathrm{min}$, which generally resulted in a processing time of 12 minutes for the $2 \mu \mathrm{m}$ thick silicon device layer in previous die etch method. For the wafer etch procedure, the etch rate varies from the center to the edge due to the etch area increasing dramatically (60 times area bigger than previous method). The purple silicon dioxide was visible first in the center of wafer area, and then extended to the edge of the wafer as the etching time increasing. The etching process is repeated for an additional 2 minutes until the silicon dioxide was completely visible for the whole wafer which resulted in a processing time of 
about 18-20 minutes for the whole wafer DRIE. After etching, the SOI die substrates were imaged and measured in an SEM. Note there is also an important distinctions between this approach and the original fabrication: the process can be performed on an entire wafer rather than on a single die.

The wafer is then diced using a dicing saw before the beams are released via wet etching; beam fabrication could be completed on the entire wafer at once but it is anticipated that subsequent sawing would likely lead to damaged beams. The die is then washed with acetone to both clean it and remove the DRIE mask; note that the original method does not remove the iron mask layer after DRIE. The cantilever beams are then released from the substrate through an isotropic buffered oxide wet etch that eliminates the silicon oxide layer below the beams; this step is timed to leave the silicon oxide below the electrical leads and bonding pads largely intact, thereby electrically isolating each beam device from one another. After rinsing the die, critical point drying using $\mathrm{CO} 2$ is performed using a SAMDRI-PVT-3D system; this prevents the cantilever beams from sticking to the substrate (as they tend to do if rinsing alone is used).

\subsection{Improved Fabrication Methods - Results}

One device using the improved fabrication method is shown in Figure 11 with a detailed side view of a beam shown in Figure 12. The free end of each microcantilever beam is separated from its associated driving electrode by a small gap (1-2 microns). The fixed end of the beam is supported by two silicon legs; in some cases, the legs form a $\mathrm{z}$ line which is the asymmetric base configuration designed to improve device sensitivity. Like the cantilever beam, the support legs are also freely-suspended above the silicon 
handle layer; the legs are mounted between two sensing electrodes that assess beam motion via piezoresistive changes across the support legs.

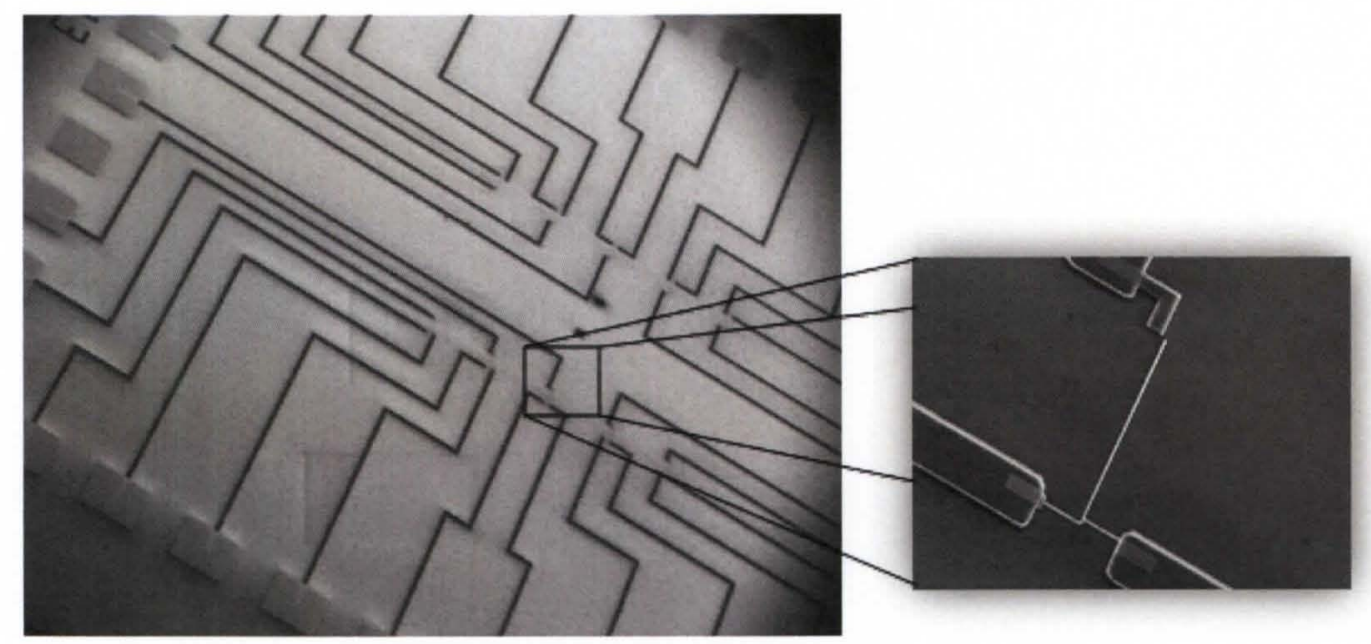

Figure 11. SEM image of single die - top view
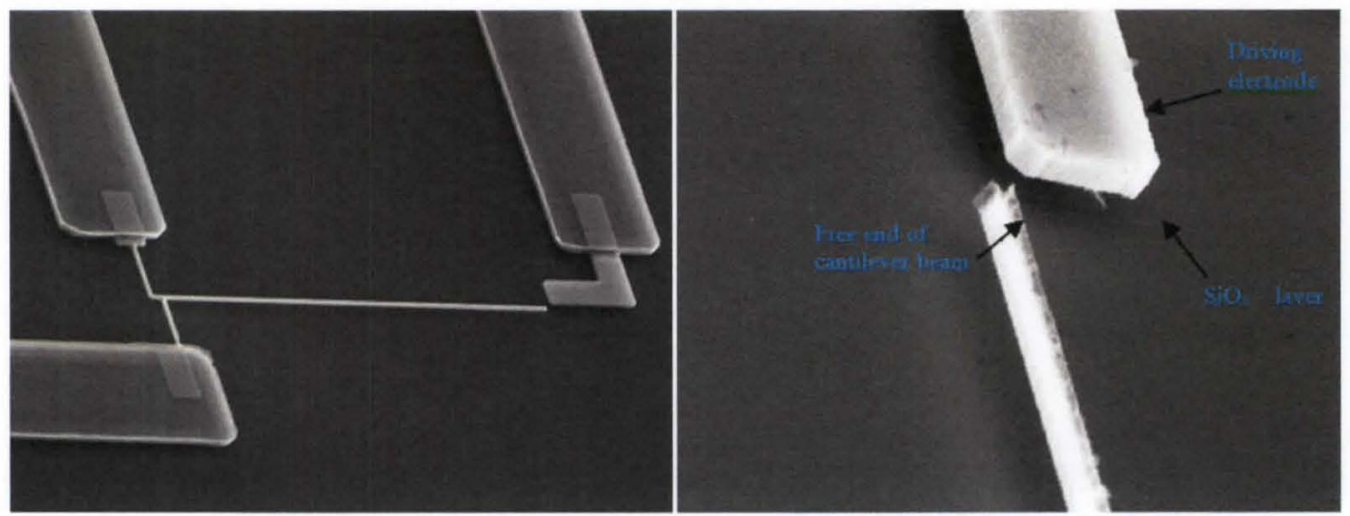

Figure 12. SEM image of single beam $-3 \mathrm{D}$ view and close-up view of beam tip.

The findings above demonstrate that the new fabrication method is capable of creating microcantilever beam devices that perform in a comparable fashion as those using the original method. This is accompanied by a dramatic reduction in fabrication 
time as demonstrated in Table 1 . The reductions in fabrication time are largely obtained in the beam pattern step (step 3 of Figure 7(b)); a single mask is used for whole wafer lithography via traditional methods.

\begin{tabular}{|c|c|c|c|c|c|}
\hline \multirow{2}{*}{\multicolumn{2}{|c|}{ Procedure }} & \multicolumn{2}{|c|}{ Time - Original Method } & \multicolumn{2}{|c|}{ Time - Improved Method } \\
\hline & & $\begin{array}{l}\text { Single } \\
\text { Device }\end{array}$ & $\begin{array}{l}\text { Whole } \\
\text { Wafer }\end{array}$ & $\begin{array}{l}\text { Single } \\
\text { Device }\end{array}$ & $\begin{array}{l}\text { Whole } \\
\text { Wafer }\end{array}$ \\
\hline \multicolumn{2}{|c|}{ Electrical Lead Writing } & $N / A$ & 3 & $N / A$ & 3 \\
\hline \multicolumn{2}{|c|}{ Bold Pad Writing } & $N / A$ & 3 & $N / A$ & 3 \\
\hline \multirow{3}{*}{$\begin{array}{l}\text { Beam } \\
\text { Pattern } \\
\text { Writing }\end{array}$} & $E$-Beam & 2 & 120 & $\overline{N / A}$ & $N / A$ \\
\hline & $\begin{array}{l}\text { Iron } \\
\text { Evaporation }\end{array}$ & 2 & 120 & $N / A$ & $N / A$ \\
\hline & $\begin{array}{l}1805 \\
\text { Photoresist }\end{array}$ & $N / A$ & $N / A$ & $N / A$ & 0.2 \\
\hline \multicolumn{2}{|l|}{$\overline{D R I E}$} & 2 & 120 & $N / A$ & 2 \\
\hline \multicolumn{2}{|c|}{ Wet Etch } & 1 & 60 & 1 & 60 \\
\hline \multicolumn{2}{|c|}{ Critical Point Dry } & 1 & 60 & 1 & 60 \\
\hline \multicolumn{2}{|c|}{ Total Fabrication Time } & 14 & 486 & 8.2 & 128.2 \\
\hline
\end{tabular}

Table 1. Fabrication time (hrs) for original and improved fabrication methods.

\subsection{Improved Fabrication Methods-Discussion}

Fabricated devices were checked in the SEM to verify both that the beams are as desired and that they are freely suspended above the handle layer. Beam widths of 12 devices are listed in Appendix A. The average width of beam for each device is shown in Table 2. It is obvious to see that the width of beam is not close to designed value in some 
way. One of the beams is demonstrated in Figure 13, which is measured in SEM for its width. The reason of beam width variation needed to be investigated.

\begin{tabular}{|c|c|c|}
\hline Device number & Designed width & Average width \\
\hline $1 \#$ & $1.1 \mu \mathrm{m}$ & 0.664 \\
\hline $2 \#$ & $1.3 \mu \mathrm{m}$ & 1.060 \\
\hline $3 \#$ & $1.3 \mu \mathrm{m}$ & 0.784 \\
\hline $4 \#$ & $1.1 \mu \mathrm{m}$ & 0.957 \\
\hline $5 \#$ & $1.7 \mu \mathrm{m}$ & 1.010 \\
\hline $6 \#$ & $1.1 \mu \mathrm{m}$ & 0.886 \\
\hline $7 \#$ & $1.7 \mu \mathrm{m}$ & 0.676 \\
\hline $8 \#$ & $1.3 \mu \mathrm{m}$ & 1.023 \\
\hline $9 \#$ & $2.1 \mu \mathrm{m}$ & 0.997 \\
\hline $11 \#$ & $1.1 \mu \mathrm{m}$ & 1.001 \\
\hline $12 \#$ & $2.1 \mu \mathrm{m}$ & 1.555 \\
\hline $14 \#$ & $1.3 \mu \mathrm{m}$ & 0.802 \\
\hline
\end{tabular}

Table 2. Average width of beam

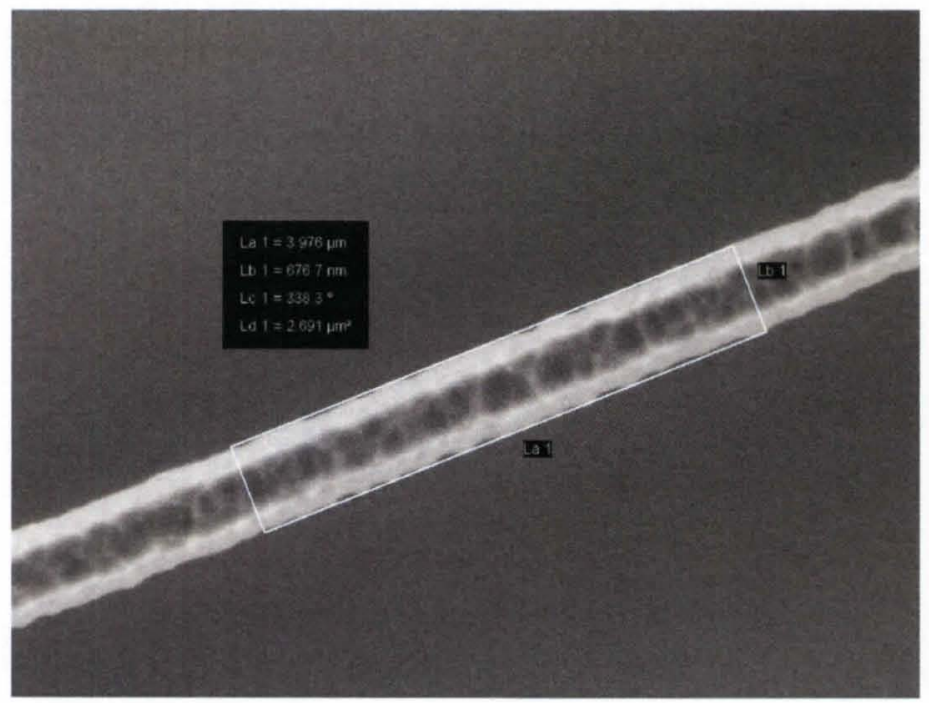

Figure 13. The beam of $1.3 \mu \mathrm{m}$ width is measured in SEM

After checking procedures step by step, two possible procedures may lead the beam to be over etched: DRIE and wet etching. The protocol is the exactly same in wet etching process as the earlier work. Therefore the first focus here is into DRIE. Etch 
depths of hundreds of micrometers can be achieved with almost vertical sidewalls in DRIE process. In the current process, Multiple ASE (Advanced Silicon Etcher) is used for DRIE where two different gas compositions alternate in the reactor. The etch cycle is as shown in Figure 14 with following steps: (1) $\mathrm{SF}_{6}$ isotropic etch; (2) $\mathrm{C}_{4} \mathrm{~F}_{8}$ passivation; (3) $\mathrm{SF}_{6}$ anisoptropic etch for floor cleaning. The $\mathrm{C}_{4} \mathrm{~F}_{8}$ creates a polymer on the surface of the substrate, and the second gas composition $\left(\mathrm{SF}_{6}\right.$ and $\left.\mathrm{O}_{2}\right)$ etches the substrate. [38]

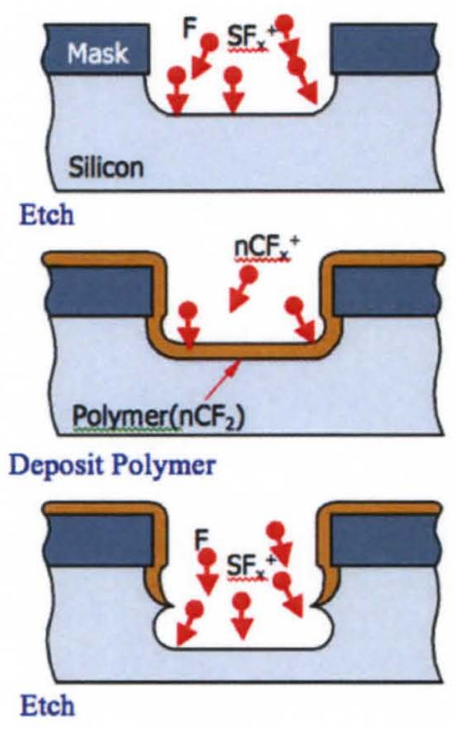

Figure 14. Illustration of how DRIE works ([39])

The protective polymer layer is deposited on the sidewalls as well as on the bottom of the etch pit; however, the anisotropy of the etch removes the polymer at the bottom of the etch pit faster than the polymer is removed from the sidewalls. As such, the sidewalls are not entirely smooth under SEM inspection. The parameters set up during DRIE should depend on the amount of exposed silicon due to loading effects in the system, with larger exposed areas etching at a much faster rate compared to smaller 
exposed areas.[39] As shown in Figure 11, the covered beam areas with photoresist are very small ( $1.1 \mu \mathrm{m}$ width and $128 \mu \mathrm{m}$ length per each beam). In the whole wafer scale, the covered beam areas are quite small compared with exposed area which needed to be etched. This could lead to great etching of the beam than desired. As the device layer is removed in the desired areas, exposed areas increase sharply compared to the initial state while etching through wafer in current process. But the parameters used in new process were kept same as used in the previous method (which only used a single dice in each DRIE step). In another words, the old etch rate of $0.17 \mu \mathrm{m} / \mathrm{min}$ may not match that needed for successful completion in the new method. The real etch rate is likely to be much faster than $0.17 \mu \mathrm{m} / \mathrm{min}$. Using the old approach, it was estimated that about 16 minutes were required to etch through the wafer; however, it likely takes less time for a wafer and results in over-etching.

To fix this issue, a new beam mask has been designed to decrease exposed areas. As shown in Figure 15(a), both pad and lead patterns are covered, and gap between dies are covered also in $3^{\text {rd }}$ mask (beam mask). More space is covered between leads as shown in Figure 15(b). In this case, the mask looks not neat and clear but it decreased exposed areas as much as possible. After determine the proper etching rate for etching through wafer, the etch must then be characterized for the exact mask feature and depth to obtain desirable results. According Liu's results[40], the parameters of etching cycle time, platen power, $\mathrm{SF}_{6}$ flow rate, ramp time, and over time effect the sidewall roughness should all be checked and adjusted to effectively improve the roughness. New beam dimensions are designed for each beam with changing width, length and shape of the beam. New beam dimensions for each die are listed in Appendix B. The bond pad label is 
added to distinguish each other in order to convenient operation in testing. The new mask and the parameters for DRIE process could be performed when funding is available.

(a)
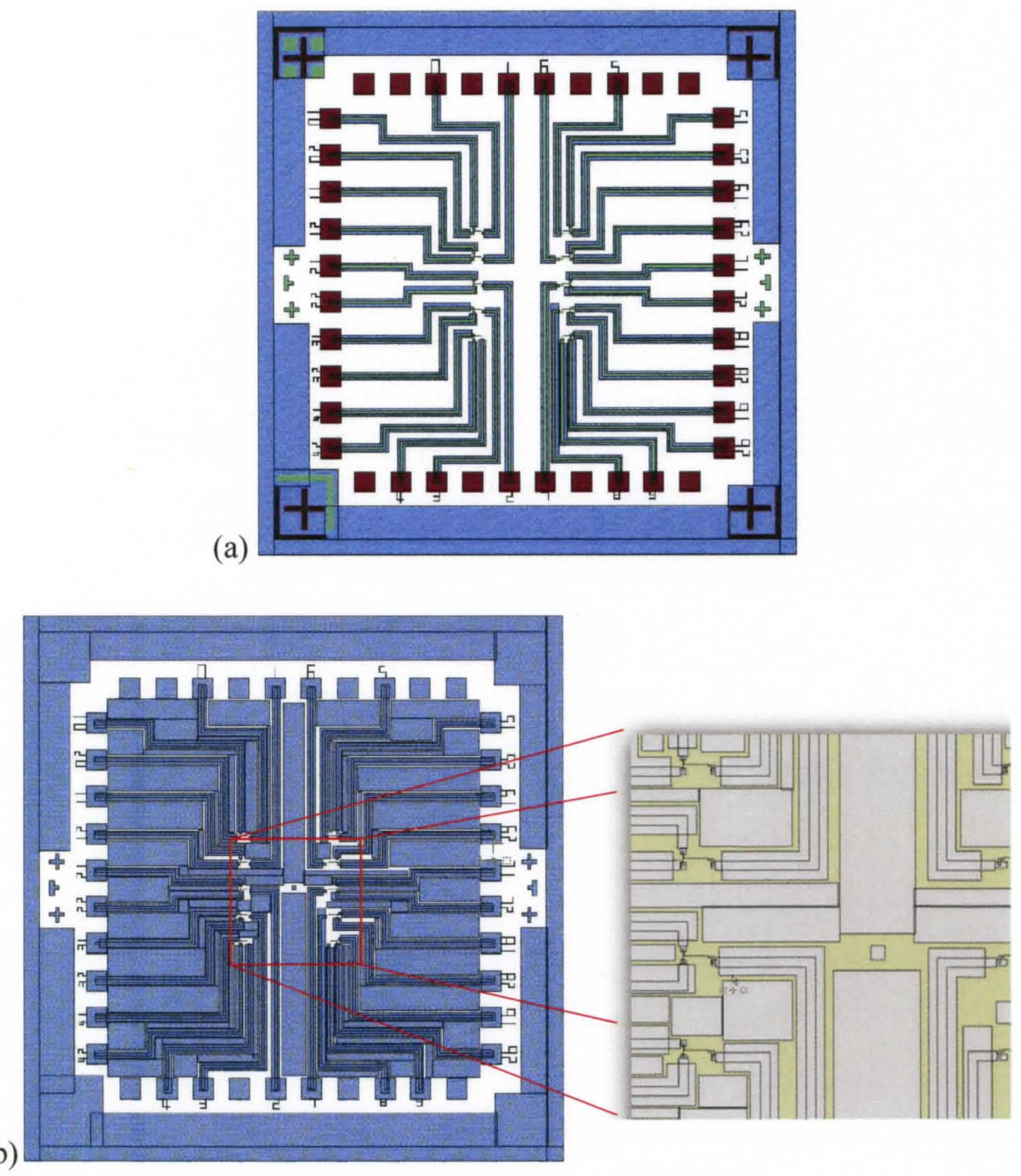

Figure 15. New beam mask to decrease exposed areas 


\subsection{Operation in Vacuum - Test Setup}

Once the beams on a single die have been created, the die can be placed in a probe station for testing. The probe station can operate either under a vacuum or with a specified atmosphere; all results in this Chapter take place under vacuum. The experimental setup is shown in Figure 16. The three probe tips are placed in contact with the driving electrode $(\mathrm{A})$ and the beam base electrodes $(\mathrm{B}-\mathrm{C})$; these are then wired to the various components as shown. The function generator applies an $\mathrm{AC}$ voltage to the driving electrode of specified value and frequency; this signal serves as the reference for the lock-in amplifier. The DC power supply places a voltage across a series circuit consisting of the beam base and a fixed resistor (14 $\mathrm{k} \Omega$ in Figure 16; varies depending on experimental setup). As the beam vibrates, stresses are induced in the beam base that depends on the displacement of the beam; this causes a change in the electrical resistance in the beam base resistance due to piezoresistive effects. The voltage at point is then provided to the lock-in amplifier as a signal to be analyzed; depending on the setting, the lock-in amplifier seeks for an $\mathrm{AC}$ signal in this input that is at frequency $1 \mathrm{f}$ or $2 \mathrm{f}$ where $\mathrm{f}$ is the frequency of the reference signal (the $\mathrm{AC}$ voltage at the driving electrode).

In order to determine the nature of the signal that the lock-in amplifier measures, several assumptions are made. First, the fixed resistor and the beam base are both considered as pure resistance with values $R_{f}$ and $R_{b}$, respectively, when the system is not in operation; this implies that any capacitance or inductance in both items can be neglected. As the system operates, the beam tip will experience a periodic displacement $u(t)$ which in turn induces a change in resistance of the beam base $\left(\Delta R_{b}\right)$. The details of $\Delta \mathrm{R}_{\mathrm{b}}$ determined from finite element analysis for several representative geometries are 
presented in Section 2.6; for this section, all that is developed is the lock-in amplifier voltage if $\Delta R_{b}$ is known.

This relationship can be determined using Ohm's law. The entire resitance R of the circuit across which the DC voltage is applied is $R_{f}+R_{b}+\Delta R_{b}$, which is then rewritten as:

$$
\begin{gathered}
\mu=\frac{R_{f}}{R_{b}} \quad \text { and } \quad \psi=\frac{\Delta R_{b}}{R_{b}} \\
R=R_{b}(1+\mu+\psi)
\end{gathered}
$$

where the term $\mu$ is constant and the term $\psi$ varies with beam displacement. Calling $V_{D C}$ as the DC voltage provided by the DC power supply, the voltage to the lock-in amplifier $\mathrm{V}_{\mathrm{L}}$ is obtained as

$$
V_{L}=V_{D C}\left(\frac{1+\psi}{1+\mu+\psi}\right)
$$

This equation can be rewritten as:

$$
V_{L}=V_{D C}\left(\frac{1+\psi}{1+\mu+\psi}\right)\left(\frac{1+\mu-\psi}{1+\mu-\psi}\right)=V_{D C} \frac{1+\mu+\mu \psi-\psi^{2}}{(1+\mu)^{2}-\psi^{2}}
$$

which for small changes in beam base resistance $(\psi<<1)$, the equation can be linearized by neglecting $\psi^{2}$ to find:

$$
V_{L} \approx V_{D C} \frac{1+\mu+\mu \psi}{(1+\mu)^{2}}=V_{D C}\left\{\left[\frac{1}{1+\mu}\right]+\left[\frac{\mu}{(1+\mu)^{2}}\right] \psi\right\}
$$


The first brace represents the voltage in the beam rest state $(\psi=0)$ while the second represents that change in voltage due to beam motion. Subtracting the first leads to the change in the $V_{L}$ which can be normalized by $V_{D C}$ to find:

$$
\frac{\Delta V_{L}}{V_{D C^{\prime}}}=\frac{\mu}{(1+\mu)^{2}} \psi
$$

One question about the experimental setup is the optimal choice for the fixed resistor. The most sensitive device is the one which maximizes the term that multiplies $\psi$. The value of $\mu$ that accomplishes this can be obtained by differentiating this term relative to $\mathrm{m}$ and finding the value of $\mu$ that causes this derivative to become 0 . This becomes:

$$
\frac{d}{d \mu}\left(\frac{\mu}{(1+\mu)^{2}}\right)=\frac{1-\mu}{(1+\mu)^{3}}
$$

Since $\mu$ is positive, this equation is only satisfied when $\mu=1$; therefore, the maximum change in voltage provided for the lock-in amplifier $\left(\Delta V_{L}\right)$ occurs when $R_{b}=R_{f}$.

The attached computer uses a LabView instrument to direct the function generator to sweep the driving electrode frequency in a series of steps between two frequencies; it then captures the resulting lock-in amplifier voltage signal for each frequency. The voltage obtained from the lock-in amplifier for one microcantilevers beam is shown in Figure 17; the peak of the signal occurs when cantilever beam is resonating (at approximately $33.32 \mathrm{kHz}$ ). On addition to the setup not present in earlier work is DVD recorder attached to the probe station optical microscope; this permits later review of the visual behavior of the system if desired; a typical image is also shown in Figure 17. 


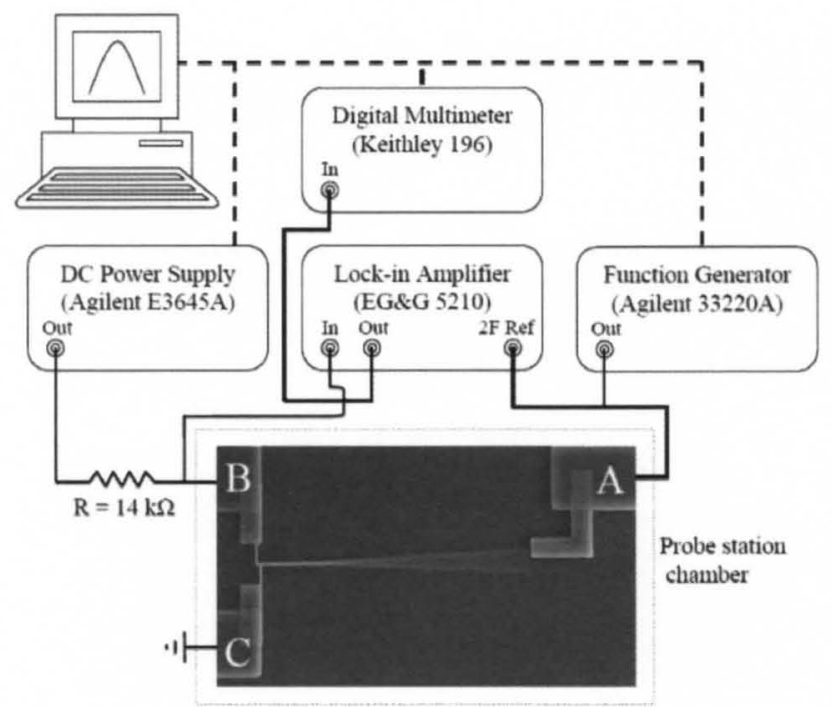

Figure 16. Schematic of general instrumentation setup.[36]

[(-) BNC Cable, (- - -) GPIB Cable]

Figure 18 shows that beam resonation is also captured using high speed digital video camera (HiSpec 2). The goal of using the Hispec 2 was to capture actual beam motion if possible. However, the light available in the probe station is not sufficient for high-speed video and the HiSpec 2 could achieve a frame rate of approximately $1 \mathrm{kHz}$, which is well below the beam frequency.

The dark dots in picture are dusts on the surface of probe station. The drive frequency swept from $31 \mathrm{kHz}$ to $35 \mathrm{kHz}$. The beam resonated while the drive frequency reached approximately $33.32 \mathrm{kHz}$. The drive frequency is input into the lock in amplifier as reference signal. The lock-in amplifier set as $f$ mode which means the signal picked up by lock-in amplifier has the same frequency with the drive frequency. The output signal caused by movement of beam, so we could say that the beam resonating frequency is same as the drive frequency. 
For each device tested, typically $6-8$ of the 10 total cantilever beams could be made to resonate; this translates to a success rate of roughly $70 \%$, with the failure cases attributed to either beam damage or other unknown causes.

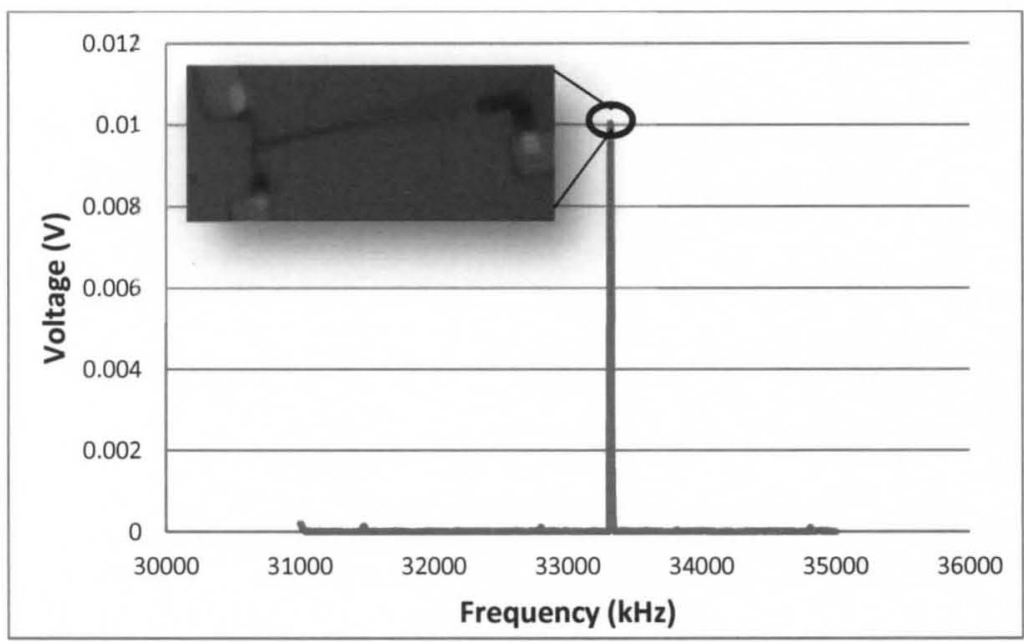

Figure 17. Voltage from lock-in amplifier during driving electrode frequency sweep.

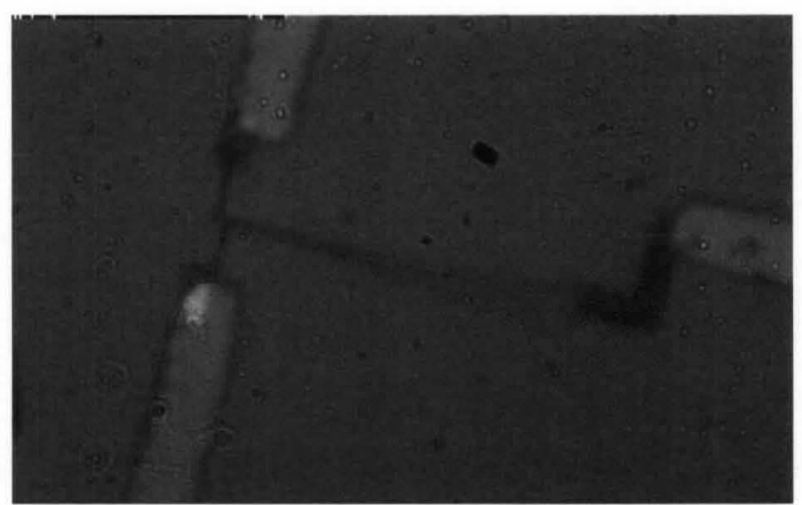

6/6/2012 1:35:22 PM -3250.1[ms] 000000003 HiSpec 2 Fastec $320 \times 198 @ 496 \mathrm{fps} 1122 \mu \mathrm{s}$ V1.0.2.0

Figure 18. Beam resonating captured with HiSpec 


\subsection{Operation in Vacuum - Beam Base Signal Frequency}

$\mathrm{Xu}$ Yang in her dissertation justified that the output signal twice the frequency of the reference in the symmetric base beams studies- since the beam is symmetric about its long axis, it provides an identical resistance change in the base whether the beam tip moves right or left. During a single resonant cycle, the beam tip moves to positions 0 (rest), $+d_{\max }, 0$ (rest), $-d_{\max }, 0$ (rest). At these same points, the resistance would become $R$ (rest value), $R+\Delta R_{\max }, R$ (rest value), $R+\Delta R_{\max }, R$ (rest value). Hence, it appears as a signal that occurs at twice the frequency of the beam motion frequency. In order to demonstrate this, the ANSYS finite element model shown in Figure 5 was modified utilized. The beam was $128 \mu \mathrm{m}$ long, $0.9 \mu \mathrm{m}$ wide with base legs each at $20 \mu \mathrm{m}$ long and symmetric (i.e. the two base legs lie along a common line). The model was assumed undamaged (i.e. the purple material has the same piezoresistive properties as the light blue material) and the tip displacement was prescribed as (in $\mu \mathrm{m})$ as $d=10 \sin (2 \pi p)$, where the parameter $\mathrm{p}$ varies from $0-1$ for a single beam resonant cycle. The associated resistance change results shown in Figure 19 indicate that the resistance frequency is twice that of the beam displacement frequency. Note that only part of the model is shown as the beam is roughly 3 times longer than the distance between the electrodes in the view below. 

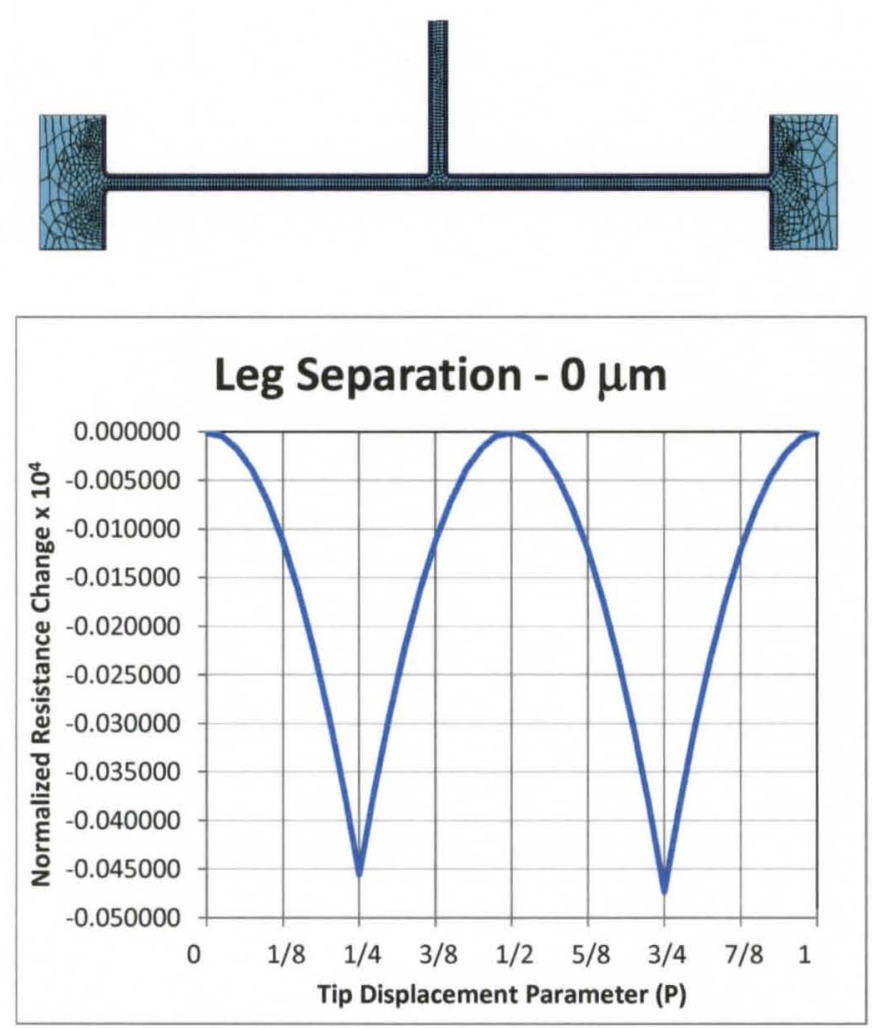

Figure 19. Beam base resistance during tip displacement cycle for symmetric case:

(top) ANSYS element model; (bottom) normalized resistance change

When Patrick Fletcher began testing asymmetric base beams, it was anticipated that the setting of the lock-in amplifier should be changed to the " $1 f$ " setting (i.e. find a signal that is at the same frequency as the reference signal). This is because the loads in the support legs in the asymmetric case form a couple to withstand the overturning moment caused by tip displacement; the sign of these loads changes depending on whether the beam has moved left or right. Therefore, the anticipated base resistance during a single beam resonant cycle would become $R$ (rest value), $R+\Delta R_{\max }, R$ (rest value), $R-\Delta R_{\max }, R$ (rest value). This is at the same frequency as the driving electrode signal. This was verified by repeating the analysis above using an asymmetric 
configuration as shown in Figure 20; the signal is clearly at the same frequency as the tip displacement. These results further demonstrate the value of the asymmetric configuration since the normalized resistance values are roughly 2 orders of magnitude greater than those for the symmetric base configuration. These calculations were repeated for a variety of base leg separation distances; the results in Figure 21 show that the signal changes from $2 f$ to $l f$ with relatively small base leg separation values (on the order of 0.01 microns).
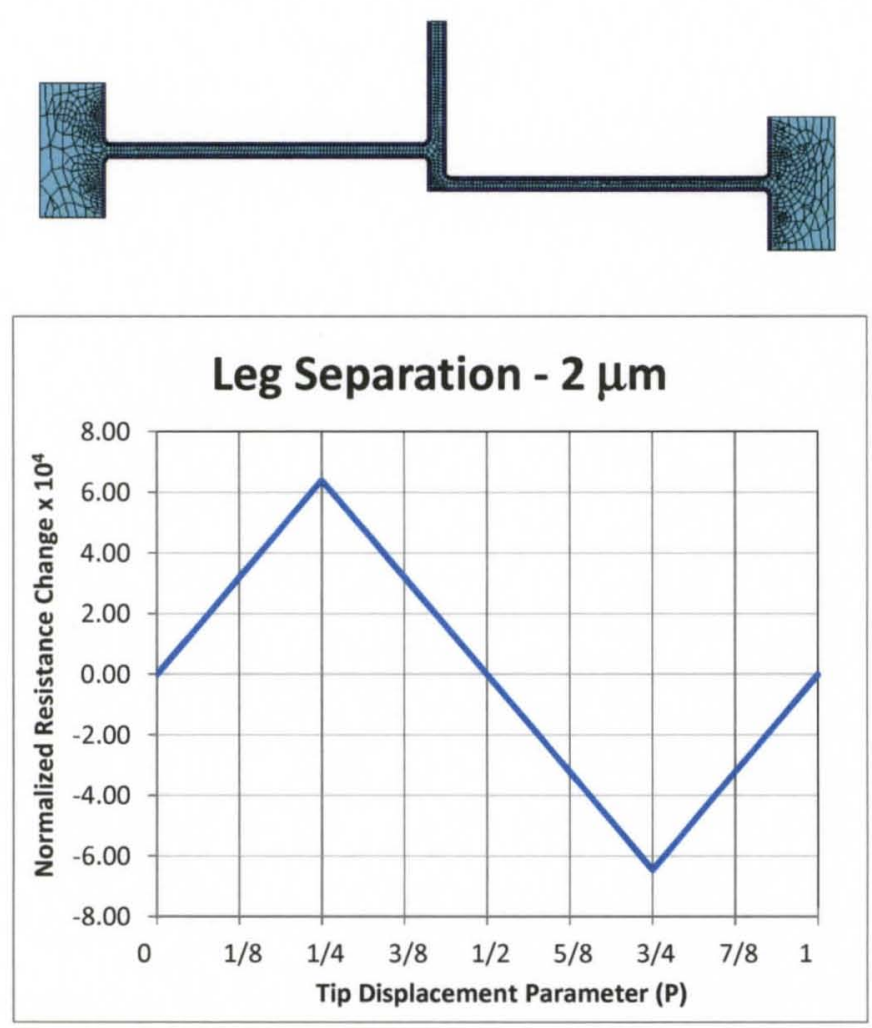

Figure 20. Beam base resistance during tip displacement cycle for asymmetric case:

(top) ANSYS element model; (bottom) normalized resistance change 


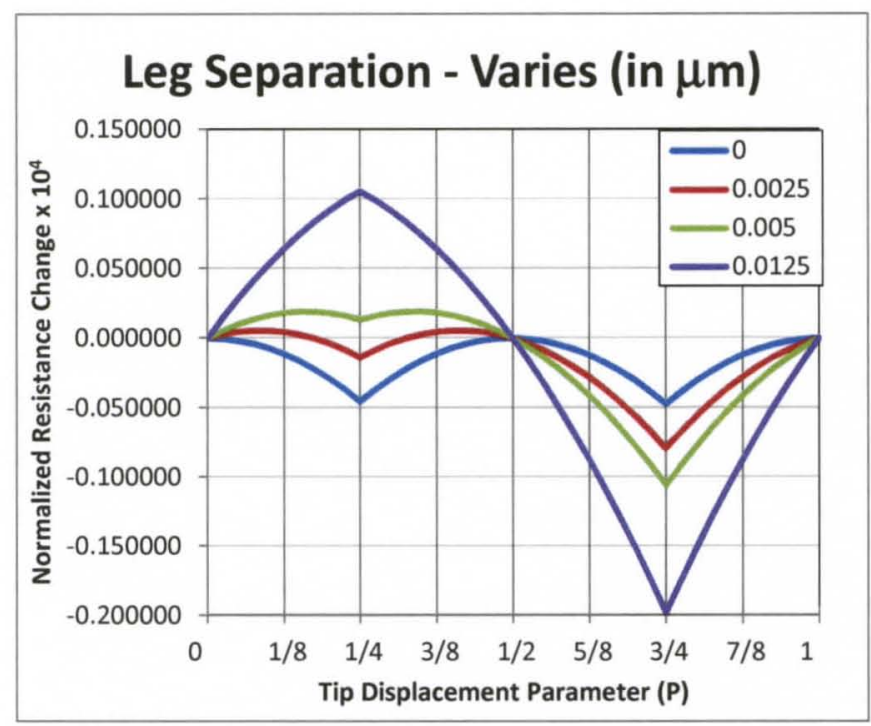

Figure 21. Beam base resistance during tip displacement cycle for asymmetric case as base leg separation is increased from $0 \mu \mathrm{m}$ to $0.0125 \mu \mathrm{m}$

The above findings would indicate that a " $1 f$ " setting is appropriate if the beam base configuration is asymmetric. The current device testing verified that the beam resonating at the drive frequency. However, in his work, Patrick Fletcher noted that the " $1 f$ ' setting often did not provide a clear a lock-in amplifier signal compared to the " $2 f$ " setting; therefore, he continued to use the " $2 f$ " setting in his work as Yang Xu had done for symmetric base beams. Also several previous devices needed " $2 f$ " setting to capture the signal. The difference during operations is silver epoxy glue adopted in current operation. In previous testing operating, the nonconductive double sided tape was used to stick device in the probe station. Currently, conductive silver epoxy glue is used to replace the tape and then beam is resonating in expected way. And the noise signal output from beam base (no matter input exists) disappeared. The conductive glue connect the handle layer grounded which works for the electrical circus. 
The beam base resistance changes shown above will be used in later analyses to simulate the voltage provided to the lock-in amplifier for analysis. For this purpose, it is preferable to plot the normalized base resistance change versus tip displacement. These are shown for the symmetric and asymmetric ( $2 \mu \mathrm{m}$ leg separation cases in Figure 22$)$. The functions describing the behavior below can be most easily expressed by breaking the responses into odd and even functions as:

$$
\begin{aligned}
& R_{\text {odd }}(u)=-R_{\text {odd }}(-u) \rightarrow R_{\text {odd }}(u)=\frac{R(u)-R(-u)}{2} \\
& R_{\text {even }}(u)=R_{\text {even }}(-u) \rightarrow R_{\text {even }}(u)=\frac{R(u)+R(-u)}{2}
\end{aligned}
$$

where $R(u)$ is the normalized beam base resistance change which can be expressed as the sum of the odd and even functions above (with appropriate sign change for the $-\mathrm{u}$ region). The odd and even functions along with trendline curve fits are show in Figure 23 and Figure 24, respectively. These plots show a couple of surprising features. First, the asymmetric beam case still has an even behavior to it that is almost of identical magnitude as the symmetric beam case; it seems reasonable that the difference is due to the longer current path in the asymmetric case but this is just speculation. Similarly, the symmetric beam case has an odd component to it but it is very small and is nearly 4 orders of magnitude smaller than the asymmetric beam case. 

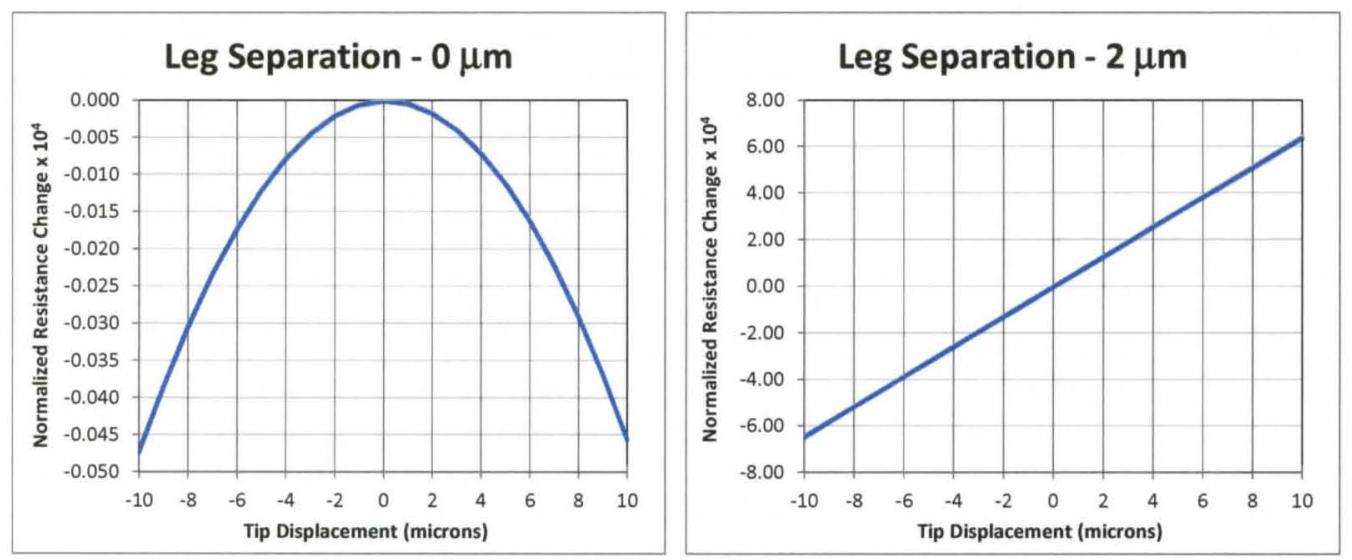

Figure 22. Normalized beam base resistance versus tip displacement: (left) symmetric case; (right) asymmetric case with $2 \mu \mathrm{m}$ leg separation
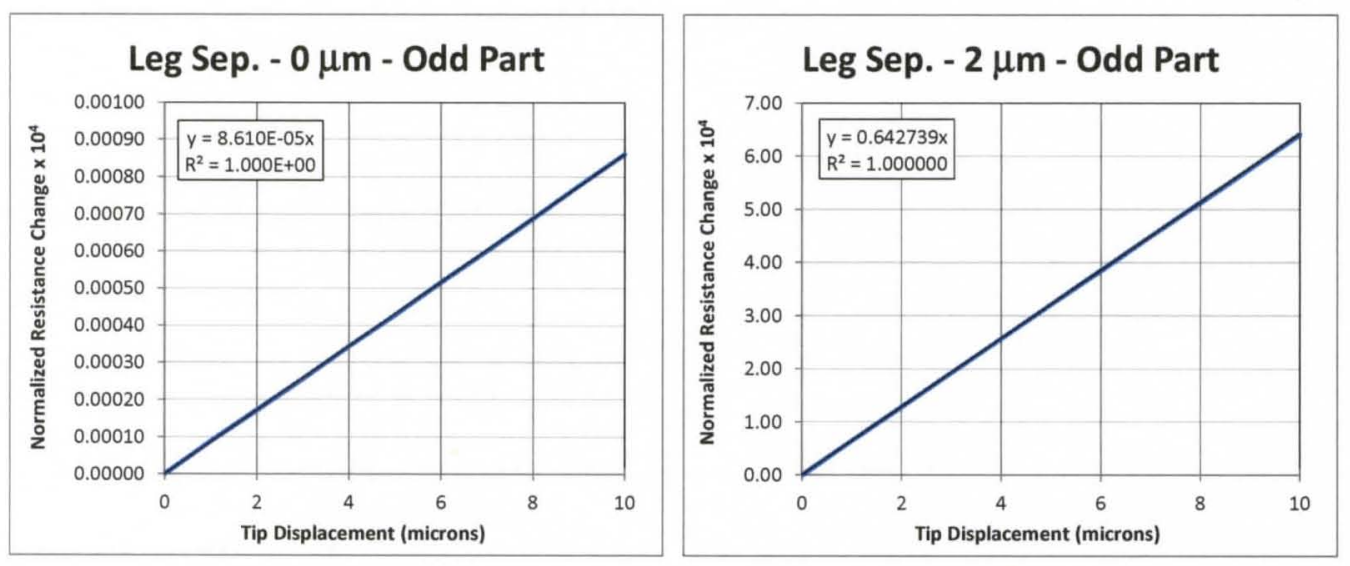

Figure 23. Odd portion $\mathrm{R}_{\mathrm{odd}}(\mathrm{u})$ of normalized beam base resistance versus tip displacement: (left) symmetric case; (right) asymmetric case with $2 \mu \mathrm{m}$ leg separation 

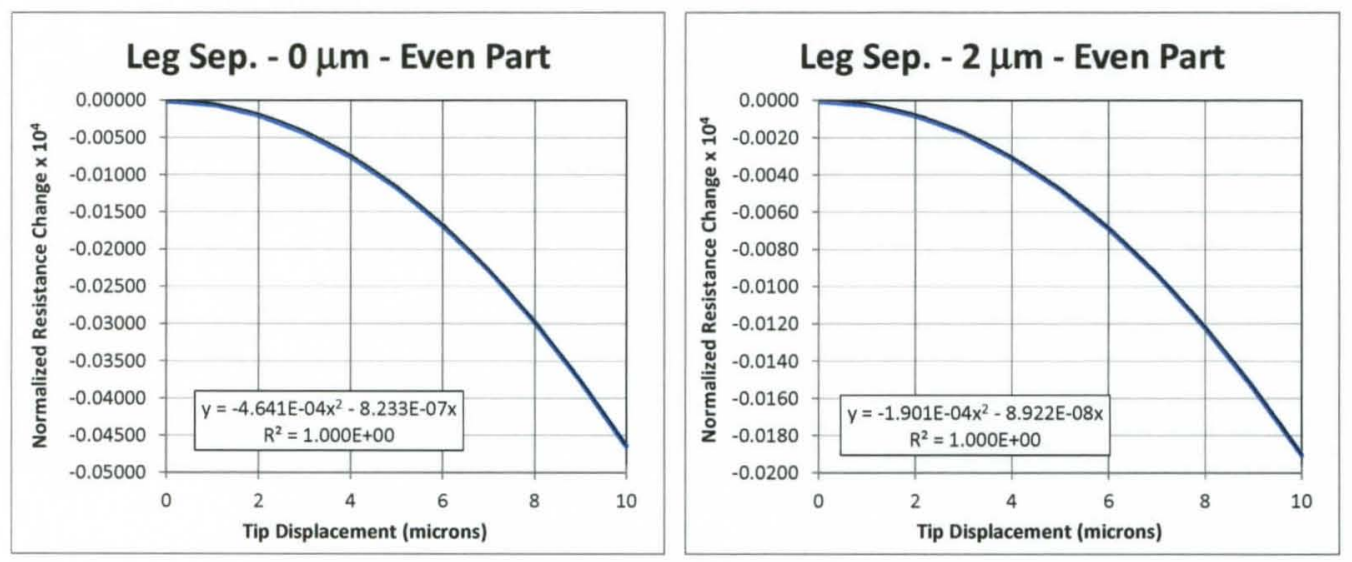

Figure 24. Even portion $\mathrm{R}_{\text {even }}(\mathrm{u})$ of normalized beam base resistance versus tip displacement: (left) symmetric case; (right) asymmetric case with $2 \mu \mathrm{m}$ leg separation

The normalized beam base resistance change (call this $R_{S}$ ) can now be specified for the symmetric beam chase as:

$$
R_{S}(u)=\left(8.610 \times 10^{-9} u\right)+\left(-4.641 \times 10^{-8} u^{2}-8.233 \times 10^{-11}|u|\right)
$$

where the first parentheses is the odd function, the second parentheses is the even function, $\mathrm{u}$ is the beam tip displacement in microns, and the $10^{4}$ scale factor in the $\mathrm{y}$ axis of the earlier plots has been used to scale the trendline coefficients appropriately. The asymmetric beam case for $2 \mu \mathrm{m}$ leg separation (call this $\mathrm{R}_{\mathrm{A}}$ ) is similarly obtained as:

$$
R_{A}(u)=\left(0.6427 \times 10^{-4} u\right)+\left(-1.901 \times 10^{-8} u^{2}-8.922 \times 10^{-12}|u|\right)
$$

The actual beam base resistance is then calculated by adding 1 to the appropriate term $\left(\mathrm{R}_{\mathrm{S}}, \mathrm{R}_{\mathrm{A}}\right)$ and then multiplying by the beam base resistance in the rest position. This will be used in Chapter 5 to predict the signal provided to the lock-in amplifier for assessing the beam vibration response near resonance. 


\subsection{Operation in Vacuum - Beam Base / Driving Electrode Leakage}

Another issue that arose during testing was apparent leakage between the driving electrode and the beam base. This should not occur since both the driving electrode and the beam are separated from one another by the insulating silicon oxide layer on which each rests. However, if the oxide layer was not functioning effectively as an insulator, signal leakage from the driving electrode to the beam base could occur.

Electrical measurements were taken using a DC circuit between various locations on several devices. The device was mounted in a probe station and each of two probes was touched to the location of interest (A, B, C, D or E) as shown in Figure 25. A series circuit was then created consisting of a fixed resistor $\left(\mathrm{R}_{\mathrm{Fixed} \mathrm{Resistor}}\right)$ and the resistance

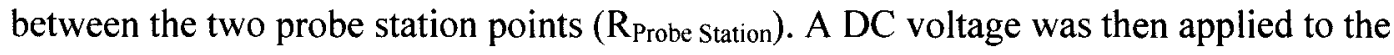
circuit and the current flow in the system was measured (using voltage drop across the fixed resistor R). Figure 25 shows the current flow results for one device for probe station pairs A-B (beam base), B-C (beam base to driving electrode), A-D (beam base to handle silicon layer) and C-D (driving electrode to silicon handle layer) as a function of applied voltage. As expected, the current across A-B is fairly linear and consistent with the resistance across the beam base in the silicon device layer. For the remaining cases, any current flow must pass through the oxide layer (separates A, B from D, E and C from D, E). If the oxide layer were undamaged, it would be expected that no current would flow. However, at larger voltages, current flow is clearly evident. Thus, there is an electrical pathway between the driving electrode and the beam base that should not be present; this is the likely source of electrical noise evident. 

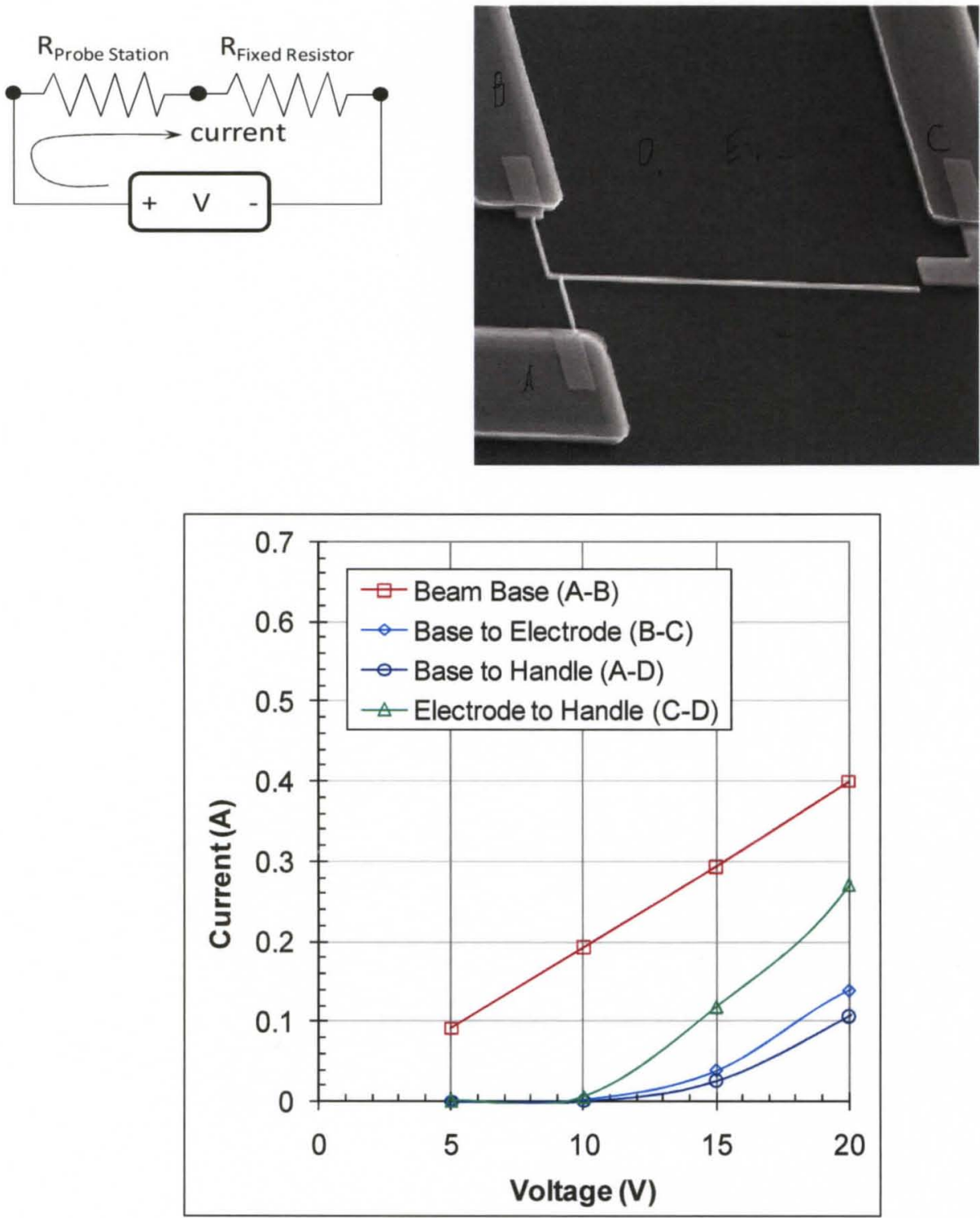

Figure 25. Device resistance measurements: (lower left) SEM image of beam with labels A-D; (upper left) DC circuit consisting of resistance between probe station tips and fixed resistor; (right) current observed in circuit (should be 0 for all except A-B if oxide is not damaged) 
At some point, the voltage across the oxide layer is sufficient to cause it to break down. This value is typically on the order of $12 \mathrm{MV} / \mathrm{cm}$.[41] For devices in this dissertation, the oxide layer is 2-3 microns thick which would indicate a need for 2400 $3600 \mathrm{~V}$ to cause spontaneous oxide breakdown. As the voltages in Figure 25 are two orders of magnitude lower than this, it is unlikely that the current flow is being caused by the applied voltage.

One possible source for the degradation in the oxide layer is the DRIE process. In this approach, ions are created that bombard the surface of the wafer that etch the silicon material away (sulphurhexaflouride $\left(\mathrm{SF}_{6}\right)$ gas for devices created at UofL). As etching occurs, a passivating gas protects the sidewalls of the silicon to prevent further lateral etching (octafluorocyclobutane $\left(\mathrm{C}_{4} \mathrm{H}_{8}\right)$ for devices created at UofL). A schematic is shown in Figure 26. At present, the DRIE process is stopped based on visual monitoring - when the silicon oxide layer is reached, a perceptible color change is evident and the process is stopped. However, it is possible that the ions may damage the oxide layer by creating an electrical pathway through it; this would perhaps occur due to the net charge imbalance between the silicon handle layer (bottom of silicon wafer) and the oxide layer exposed to ion bombardment. 


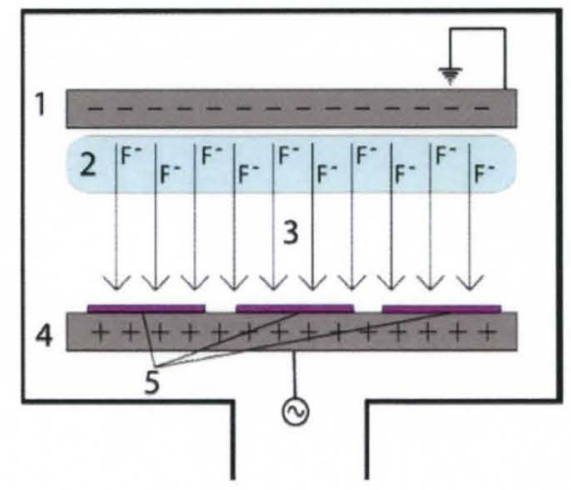

Figure 26. A schematic of the DRIE process: charged parallel plates $(1,4)$ are used with a gas (2) to create ions that bombard (3) the silicon wafer (5)

[from http://en.wikipedia.org/wiki/File:Riediagram.gif]

At this point, it is uncertain if the oxide layer is experiencing a breakdown but no other sources have been identified for the signals seen above. If it is caused by the DRIE process, an alternate approach to the DRIE setup might prevent it. One idea is to electrically connect the device layer so that as DRIE progresses there remains an electrical path from the handle layer to ground other than through the oxide layer. Another one is discussed in Section 2.4 to decrease the exposure area in DRIE to avoid the overetch. 


\subsection{Operation in Vacuum - Avoidance of Stiction}

After DRIE and rinsing, critical point drying is used to eliminate the rinse agent. This step avoids a problem called "stiction" in which the beam tips are attached to the handle layer once drying is complete. In this configuration, the beam cannot be made to vibrate and it is essentially useless as a sensor.

One of the findings from the beams resonating in vacuum is that the beam often ends in a state of stiction once the operation is concluded. This occurrence also seemed more frequent as the DC voltage applied to the beam base was increased to voltages in the range of $20 \mathrm{~V}$. It is believed that stiction in this case is caused by electrostatic forces between the beam tip (at the same voltage as the center of the beam support base) and the handle layer (not grounded, uncharged) as the experiment is terminated.

If this is the explanation, one possible remedy is to place the beam and the handle layer at a common voltage; this is shown in Figure 27 assuming a voltage level of 5 volts. This can be accomplished by electrically contacting the handle layer and placing it in the DC circuit with the beam base such that the voltage level of the beam tip and the handle layer are approximately equal at all times. This alternate configuration will be tested in future studies see if it results in fewer stiction cases after testing.

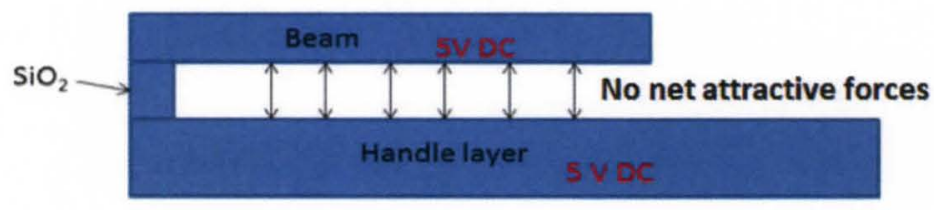

Figure 27. Schematic of proposed operation with charged handle layer to reduce the likelihood of stiction. 


\section{CHAPTER 3. EFFECT OF SUUROUNDING FLUID ON BEAM BEHAVIOR}

The goal of the devices pursued in this study is to ultimately detect and identify gases based on the observed resonance changes in the beam. In order to do so, a correlation between fluid properties and associated beam resonance changes is required. In this chapter, forces from the gas on the resonating beam are considered using both computational fluid dynamic simulation and analytical models. Once identified, these forces can be used with classical vibration models to predict beam resonance behavior. During operation, the gas properties that best agree with the observed physical beam behavior could then be calculated, with the expectation that these will be beneficial to identify the gas.

\subsection{Overview of Vibrating Microcantilever in a Gas Environment}

The fundamental structure of the sensors described in this dissertation is a microcantilever beam in a state of resonance. A schematic of such a beam is shown in Figure 28. In the current system, the driving electrode provides periodic electrostatic loading to the beam that causes resonance at proper frequencies. These forces are largely

located near the beam tip and can be expressed any number of ways; further evaluation of this question is considered in Section 4.2 on electrostatic force modeling. In Figure 28, the electrostatic loading is considered as a transverse force $\left(F_{d}\right)$ at the end of the beam; it could also include a beam tip moment (not shown). 

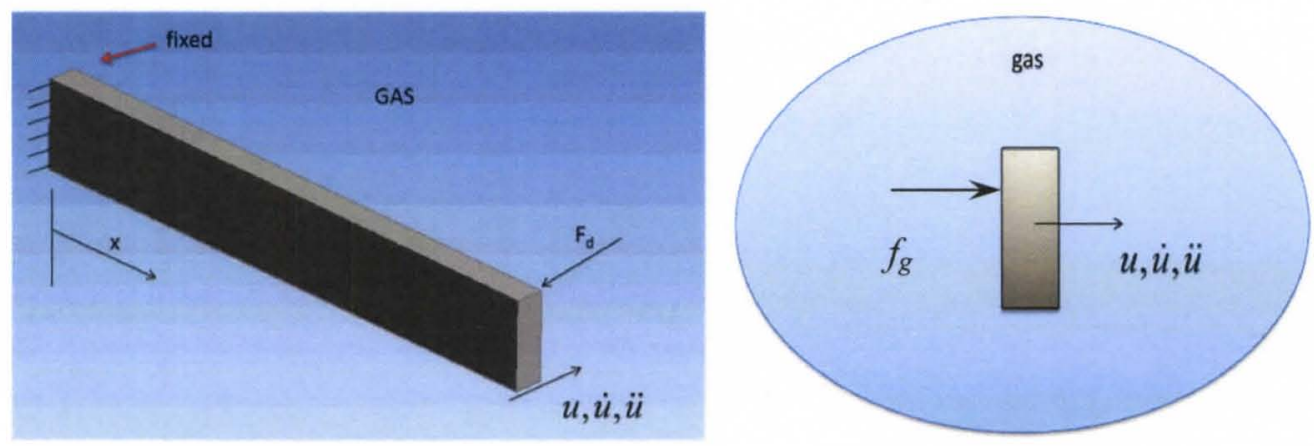

Figure 28. Schematic of cantilever beam resonating in a gas: (left) 3D view of beam with tip load $\mathrm{F}_{\mathrm{d}}$; (right) section view of beam at location $\mathrm{x}$ along beam with local distributed $\operatorname{gas} \operatorname{load} \mathrm{f}_{\mathrm{g}}$.

For the vibration problem, the beam is considered to translate in the plane defined by the beam and the sensor base (i.e. the silicon device layer). It is also assumed that the electrostatic load and the load due to gas viscous and inertial effects are in the same plane. Utilizing the simplifications of a beam in pure bending, the motion of the entire beam shown in Figure 28 can be considered by a single function $u(x, t)$, which is the lateral displacement of the point at the centroid of the beam at location $x$ at time $t$. The velocity and acceleration of a segment of the beam at the same location can be obtained as $\dot{u}(x, t)$ and $\ddot{u}(x, t)$, respectively, where the dots indicate time differentiation $(\partial / \partial t)$. For the model in Figure 28 for a beam of length L, the beam end conditions can be specified as boundary conditions, namely:

$$
\begin{array}{ll}
u(0, t)=u^{\prime}(0, t)=0 & \text { Fixed at base }(x=0) \text { no translation or rotation } \\
E^{\prime} u^{\prime \prime}(L, t)=0 & \text { Moment at beam tip is } 0(x=\mathrm{L}) \\
\operatorname{EI}^{\prime \prime \prime}(L, t)=F_{d} & \text { Shear is at beam tip }(x=\mathrm{L})
\end{array}
$$

where the ' symbol indicates spatial differentiation $(\partial / \partial x)$. 
The equation of motion for a segment of the beam at location $\mathrm{x}$ can be derived as:

$$
m_{B} \frac{\partial^{2} u}{\partial t^{2}}+E I \frac{\partial^{4} u}{\partial x^{4}}=f_{d}(x, t)+f_{g}(x, t)
$$

where $m_{B}$ is the beam mass per unit length, $\mathrm{E}$ is the modulus of elasticity of the beam material, I is the moment of inertia for the beam bending in the plane of interest, $f_{g}$ is the force from the surrounding gas (per unit length), and $f_{d}$ is an externally applied distributed load (per unit length). In the case of the beam considered here operating in vacuum, $f_{g}=f_{d}=0$ and the motion reduces to one of an undamped beam in a state of driven vibration (by tip force $F_{d}$ ).

In order to characterize beam vibration in the presence of a gas, an understanding of the gas loads during vibration is required; to accomplish this, a model to predict the fluid flow around the beam must first be undertaken. In the most general sense, this would require a $3 \mathrm{D}$ analysis since there will be fluid flow in all directions, especially at the beam tip and base. However, the problem can be greatly simplified by making the assumption that a 2D fluid flow model would suffice. This implies that:

1) The gas motion along the beam is largely in the plane of vibration.

2) The out-of -plane gas motion at the tip and gas motion related at the base is small and can be neglected.

3) The beam forces due to out-of -plane gas motion are small and can be neglected.

Whether this is a good set of assumptions can be addressed using a 3D computational fluid dynamics (CFD) simulation. This is beyond the scope of this dissertation. However, 
it should be noted that other researchers $[22,42]$ have simulated beam response using models based on similar assumptions (e.g. string of vibrating spheres model).

In the $2 \mathrm{D}$ modeling scenario, each segment $d x$ of the beam at location $x$ can be treated as experiencing a distributed gas forces $f_{g}$ that is equivalent to an infinitely long beam undergoing the same motion as the segment. This permits the gas load to be assessed by $2 \mathrm{D}$ analytical and computational fluid dynamics models.

One important non-dimensional parameter for fluid flow problems is the Reynolds number, which is defined as:

$$
\operatorname{Re}=\frac{V D}{v}=\frac{\rho V D}{\mu}
$$

where $V$ is the velocity of the flow, $D$ is a size indicating the dimension of the feature of interest, and $v$ is the kinematic viscosity, which is equal to $\mu / \rho$ where $\mu$ is the absolute (or dynamic) viscosity and $\rho$ is the fluid density. The microcantilever beams studied here represent low Reynolds number problems as can be demonstrated by a quick example. Suppose the current system is operated in air at pressure $p$ with a beam tip motion of \pm 4 microns (consistent with the resonating shape shown in Figure 3) resonating at $80 \mathrm{kHz}$. Hence the tip displacement $u_{T i p}$ (in meters) and tip velocity $\dot{u}_{T i p}$ (in meters per second) is thus given by:

$$
\begin{gathered}
u_{T i p}(t)=4 \times 10^{-6} \sin \left[2 \pi\left(80 \times 10^{3}\right) t\right] \\
\dot{u}_{l i p}(t)=\frac{\partial u_{T i p}}{\partial t}=\left[\left(4 \times 10^{-6}\right)\left(160 \pi \times 10^{3}\right)\right] \sin \left[2 \pi\left(80 \times 10^{3}\right) t\right]
\end{gathered}
$$


For air, use the standard atmosphere absolute viscosity $\mu=17.89 \times 10^{-6} \mathrm{~N}-\mathrm{s} / \mathrm{m}^{2}$ and density $\rho=1.225 \mathrm{~kg} / \mathrm{m}^{3}[43]$ at temperature $\mathrm{T}=15^{\circ} \mathrm{C}$ at and pressure $p_{0}=101.3 \mathrm{kPa}$. Assume that the value of $\mu$ applies for all pressures and that the density $\rho$ changes linearly with pressure. Further assume a dimensional size $D$ equal to the beam width (1.1 microns) and set the velocity $V$ equal to the peak velocity $(0.64 \pi \mathrm{m} / \mathrm{s})$. With this, the Reynolds number becomes:

$$
\left.\begin{array}{l}
V D=2.212 \times 10^{-6} \mathrm{~m}^{2} / \mathrm{s} \\
\rho=1.225\left(\mathrm{p} / \mathrm{p}_{0}\right) \mathrm{kg} / \mathrm{m}^{3} \\
\mu=17.89 \times 10^{-6} \mathrm{~kg} / \mathrm{m}-\mathrm{s}
\end{array}\right\} \rightarrow \operatorname{Re}=0.1515\left(\mathrm{p} / \mathrm{p}_{0}\right)
$$

The Reynolds number is thus approximately 0.15 at atmospheric pressure and reduces from there at lower pressures. The investigations in this dissertation will focus on atmospheric pressure and below for beams with dimensions and operation similar to that above. Hence, the fluid flow regime is for low Reynolds number with Re below 0.15 . The Navier-Stokes[44] equations are the basic differential equations describing fluid flow problems. For an incompressible Newtonian fluid, the Navier-Stokes equation can be written as:

$$
\rho\left(\frac{\partial V}{\partial t}+V \cdot \nabla V\right)=-\nabla p+\rho g+\mu \nabla^{2} V(16)
$$

Due to the nonlinearity arising from the convective acceleration terms $(\mathrm{V} \cdot \nabla \mathrm{V})$, there are no general analytical methods for solving the general Navier-Stokes equation.

There are, however, a few practical fluid flow problems can be solved using an exact analytical approach. First, the flow is typically assumed to be incompressible and a 
Newtonian fluid. For general problems involving slow fluid or creeping flow, which has very low Reynolds number, the approaching flow is so slow that accelerations of the fluid as it passes around the beam can be ignored. In this case, the nonlinear convective acceleration terms become small and can be neglected as:

$$
V \cdot \nabla V=0
$$

The modified Novier-Stokes equation becomes:

$$
\rho \frac{\partial V}{\partial t}=-\nabla p+\rho g+\mu \nabla^{2} V
$$

This equation can be solved analytically because it is now linear in velocity.[44] Under these low Reynolds number conditions in current study, several analytical models express the gas force on the beam in terms of the beam velocity and acceleration as:

$$
f_{g}(t)=-c_{g} \frac{\partial u}{\partial t}-m_{g} \frac{\partial^{2} u}{\partial^{2} t}
$$

where $c_{g}$ and $m_{g}$ are coefficients provided by the associated model. These coefficients are independent of velocity and acceleration; therefore, once indentified they can be used to assess drag force under a variety of motions. These coefficients could be similarly derived through comparing Stokes oscillating cylinder model with results from a computational fluid dynamics (CFD) simulation; this will be one focus of this chapter, in which geometries that are not identical to those of the analytical model considered by Stokes. This form for the gas forces has particular benefit for vibrations modeling as it allows the equations to be rearranged as: 


$$
\left(m_{B}+m_{g}\right) \frac{\partial^{2} u}{\partial t^{2}}+c_{g} \frac{\partial u}{\partial t}+E I \frac{\partial^{4} u}{\partial x^{4}}=f_{d}(x, t)
$$

This equation describe the cantilever beam in a gas environment as having an effective mass per unit length $\left(m_{B}+m_{g}\right)$ with a damping term $\left(c_{g}\right)$. It is from this equation that developments of beam resonance response will be developed in Chapter 5.

\subsection{CFX-Overview and Validation Using Taneda Results}

ANSYS CFX is a commercial computational fluid dynamics (CFD) program, used to simulate fluid flow in a variety of applications. The author and her advisor have a great deal of past experience with ANSYS products and this lead to the choice for CFX as the modeling tool for the oscillating beam problem. The oscillating beam problem requires a complicated, time-varying solution. Before starting this more ambitious study, this dissertation begins with the simulation of a simpler, steady-state CFD problem that can be compared to experimentally established behavior. Specifically, the paper "Visualization of separating Stokes flows" by Sadatoshi Taneda [45] is used as comparison; this work considers low Reynolds number flow $(\operatorname{Re}=0.010)$ around several geometries and configurations (spheres, cylinders, walls, etc.). The section below will demonstrate very similar findings between the experimental results and the ANSYS CFX results, providing confidence that the software and modeling conditions are being used appropriately.

\subsubsection{Overview of Taneda Experiments}

Two scenarios from the set of experiments presented in the Taneda paper were selected for modeling here. The simple schematic diagram of the first experiments is shown in Figure 29. Taneda used two circular cylinders with equal diameters placed 
streamwise in a uniform flow. The cylinders move horizontally at the velocity of 0.2 $\mathrm{cm} / \mathrm{s}$. The gap between the two cylinders was varied to observe the distance required to achieve flow separation. The second experiment is shown in Figure 30(a). A single cylinder is maintained at a fixed distance above a plate. The entire plate-cylinder unit moves at a constant velocity. The cylinder is sufficiently far from the plate edge that a fully-developed flow is established as shown in Figure 30(b). As in the first experiment, the gap between the cylinder and the wall was varied to observe the distance required to achieve flow separation.

In both experiments, glycerin was used as the working fluid and the tank dimensions were $100 \mathrm{~cm}$ in length, $20 \mathrm{~cm}$ in width and $20 \mathrm{~cm}$ in depth. The kinematic viscosity was about $20 \mathrm{~cm}^{2} / \mathrm{s}$, which corresponded to a Reynolds number of the order of $10^{-2}$. Reflecting particles were placed in the fluid and illuminated using lasers such that streamlines in the illuminated plane became visible. Results from these experiments will be compared to CFX modeling results below.
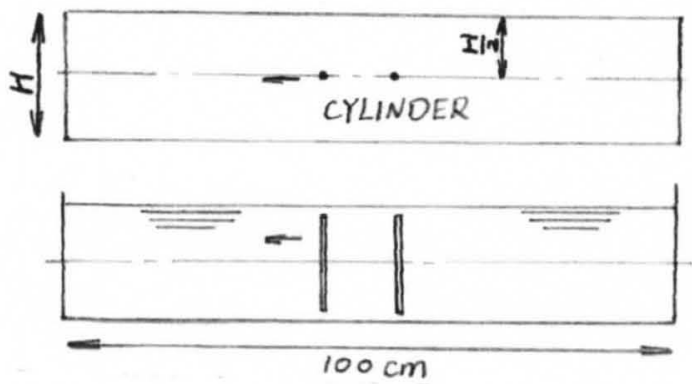

Figure 29: Schematic diagram of the experiment in which two cylinders move to the left at constant velocity: (top) view looking down the cylinder axes; (bottom) side view with the illuminated layer indicated[45] 


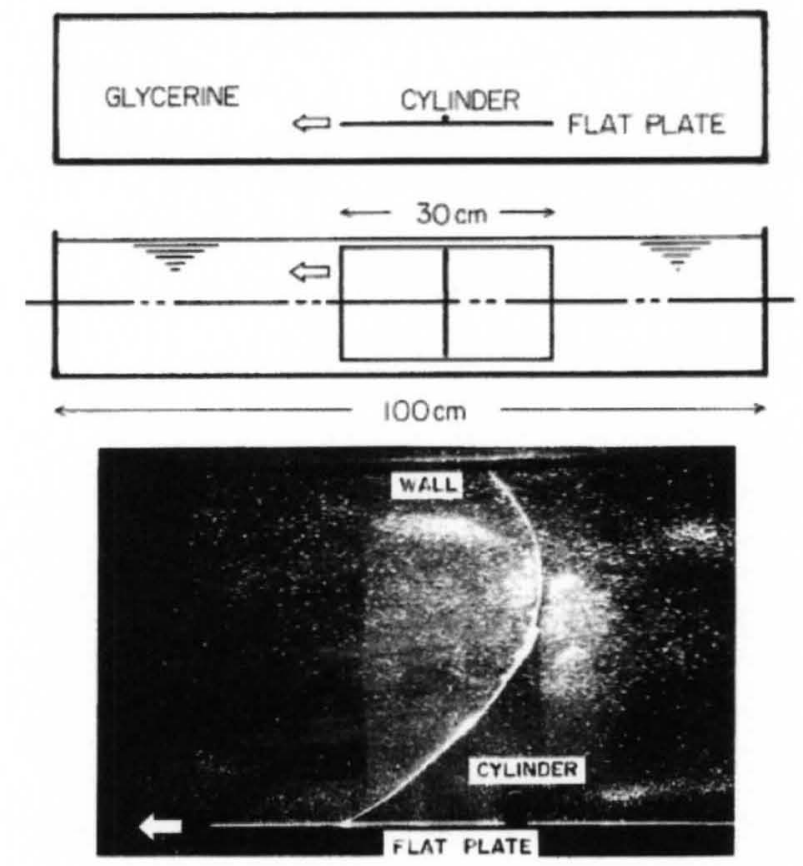

Figure 30. Schematic diagram of the experiment in which a cylinder-wall pair move to the left at a constant velocity: (left top) view looking down the cylinder axis; (left bottom) side view with the illuminated layer indicated; particles that were initially in a straight vertical line as the cylinder-plate reaches them to indicate flow regime[45]

\subsubsection{Setup in CFX - Two Cylinder Experiment}

The geometry for the two cylinder experiment consists of a rectangular glycerin tank in which the middle of the tank is interrupted by two cylinders, and is shown as Figure 31. The front and back faces of the tank (normal to the cylinder axes) are defined as symmetry boundaries; this is equivalent to stating that there are no fluid flows in the cylinder axes direction and makes the 3D problem into a 2D problem for faster solution. This method can be used when geometry, mesh and fluid flows are invariant in the 
direction normal to the symmetry surface, as can be reasonably argued is the case for these experiments. It should be noted that CFX does not contain options to directly simulate 2D problems; a 3D problem with suitable boundary conditions must be used.

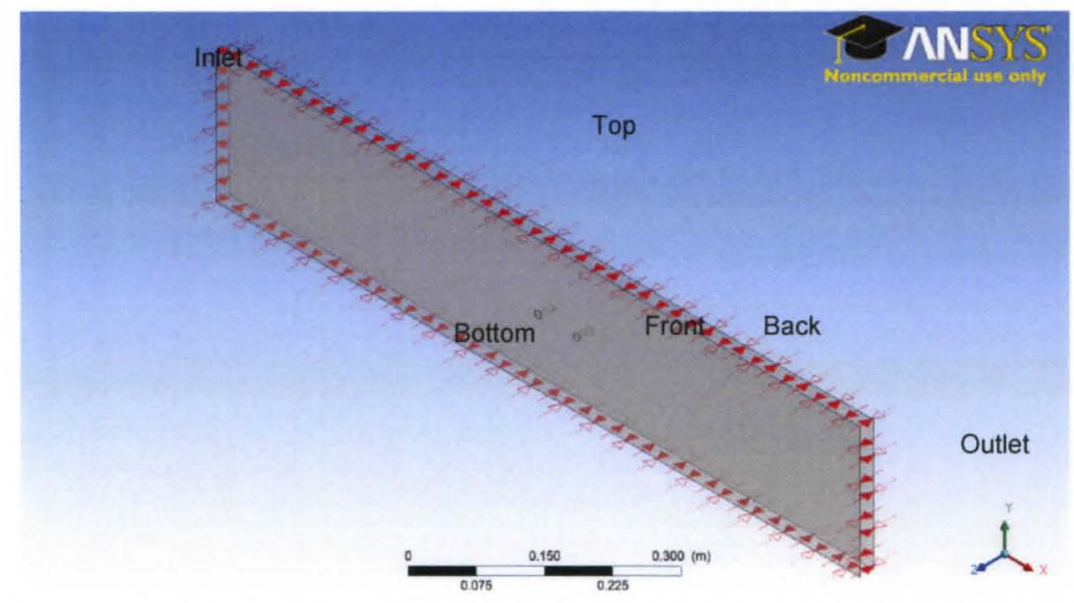

Figure 31 . The geometry consists of a $2 \mathrm{D}$ tank and two cylinders.

The height $\mathrm{H}$ of channel equals the width of the tank in Figure 29. The length of channel is $100 \mathrm{~cm}$, and the diameter D of cylinders is $1 \mathrm{~cm}$. So the channel length is $100 \mathrm{D}$ with the cylinders placed in the center; this simulates the inlet and outlet being infinitely far from the cylindrical disturbance. The two cylinders in the fluid are fixed and defined as smooth with no slip walls. In the actual experiment, the fluid is quiescent and the cylinders move at a steady speed of $0.2 \mathrm{~cm} / \mathrm{s}$. According to Panton, an identical solution to the problem is obtained if solved in terms of a coordinate system attached to the cylinder ("Section 10.7 Invariance of Incompressible Flow Equations Under Unsteady Motion").[46] In this approach, the cylinder has at rest in a fluid with a constant far field velocity of $0.2 \mathrm{~cm} / \mathrm{s}$. Hence, the fluid profile in both inlet and outlet of channel are constant at this speed, the bottom and top of the channel are defined as free 
slip walls which simulates constant velocity infinitely far above and below the cylindrical disturbance. The parameters for the fluid are shown in Table 3; the Reynolds number based on the cylinder diameter is $0.01 \mathrm{~m}$.

\begin{tabular}{|l|l|}
\hline Molar mass & $92.0938 \mathrm{~kg} / \mathrm{kmol}$ \\
\hline Density & $1261.3 \mathrm{~kg} / \mathrm{m}^{3}$ \\
\hline Specific heat capacity & $2430 \mathrm{~J} / \mathrm{kg}^{\circ} \mathrm{K}$ \\
\hline Dynamic viscosity & $2.522 \mathrm{~Pa} \cdot \mathrm{s}$ \\
\hline
\end{tabular}

Table 3. Parameters for fluid used in experiment.3.2.3 Setup in CFX - Cylinder with wall experiment

In order to simulate the experiment with a cylinder above a fixed wall, fully developed flow is chosen for defining the velocity profile in CFX. As shown in Figure 32 , directly after a pipe entrance, velocity profiles are initially almost uniform. However, viscous effects cause slowing in the velocities, beginning near the walls and then extending deeper into the flow. Finally, a fully developed laminar flow is established and will remain so thereafter; this represents a flow regime with a velocity profile that does not vary as it moves down the pipe. Using this in CFX matches the velocity profile shown in the time line image of Figure 30(b).

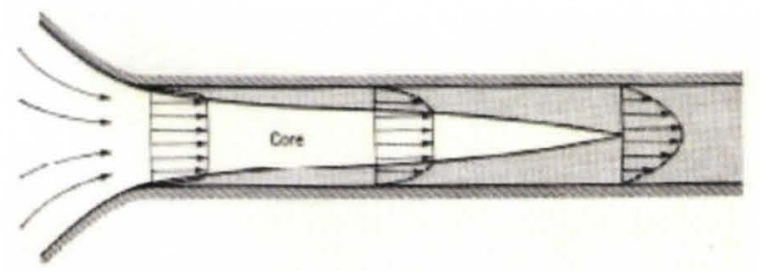

Figure 32. Boundary-layer growth at pipe entrance.[44] 
In the model, a circular cylinder is placed near the bottom of channel. The cylinder, bottom surface and top surface, shown in Figure 33, are all defined as smooth wall with no slip. Without the cylinder present, the simulation would correspond to $2 \mathrm{D}$ flow in a slot of height $\mathrm{H}$ that is infinitely deep in the out-of-plane direction. The fully developed velocity profiles in inlet and outlet that correspond to this condition are defined with equation:

$$
V=V_{\max }\left[1-\left(\frac{2 y}{H}\right)^{2}\right]
$$

where the top and bottom of the channel are at y values of $1 / 2 \mathrm{H}$ and $-1 / 2 \mathrm{H}$, respectively.

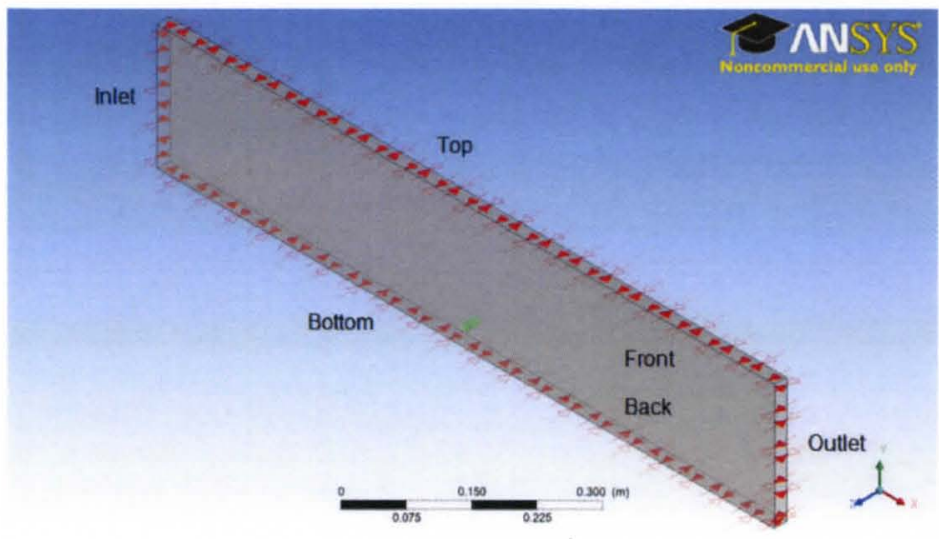

Figure 33 . The geometry consists of a 2D tank and one cylinder near bottom.

For a $2 \mathrm{D}$ planar geometry, a thickness of approximately $1 / 100^{\text {th }}$ the length of the largest dimension in the model generally provides a nice mesh with good quality cells. In our case, the thickness of tank should choose $1 \mathrm{~cm}$. Since the model is a 2D 
representation, a single cell thickness is used in the $3^{\text {rd }}$ dimension. Only the $2 \mathrm{D}$ face is meshed. The mesh result for two cylinder experiment is shown in Figure 34.

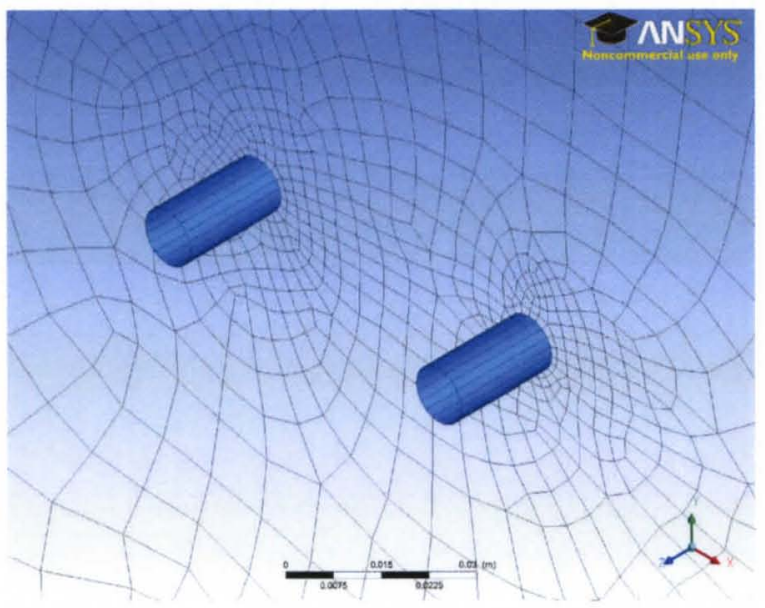

Figure 34. Mesh result of the model.

\section{$\underline{\text { 3.2.3 Results - Two Cylinder Experiment }}$}

Modeling results for fluid flows through the two fixed cylinders are listed below with gap $\varepsilon$ ranges from $1.5 \mathrm{D}, 1 \mathrm{D}, 0.3 \mathrm{D}$ and $0 \mathrm{D}$ where $\mathrm{D}$ is the diameter of the cylinder $(1$ $\mathrm{cm}$ ). Compared with the pictures captured by Taneda (shown in black and white on the left panel)[45], the current modeling arrives at excellent agreement. It is observed that, as in the experiment, the flow separation vortex clearly occurs when $\varepsilon / D$ is less than 0.5 . 
(a)
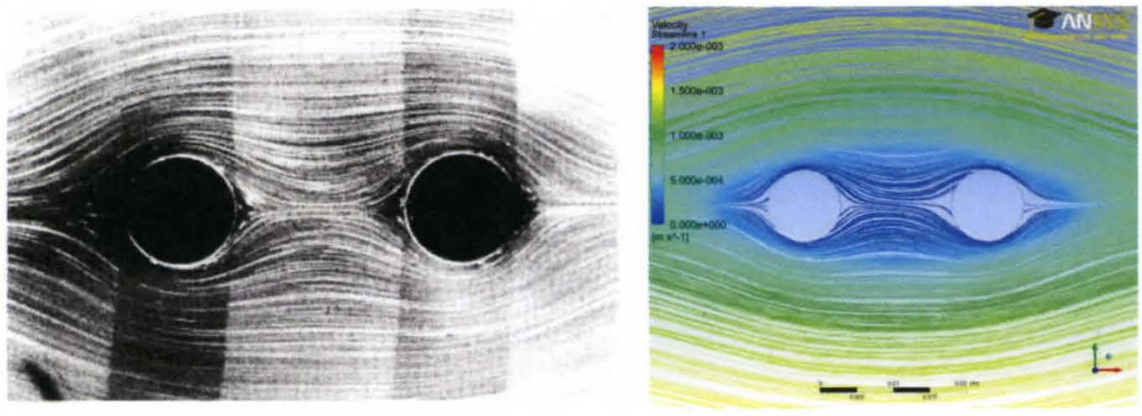

(b)
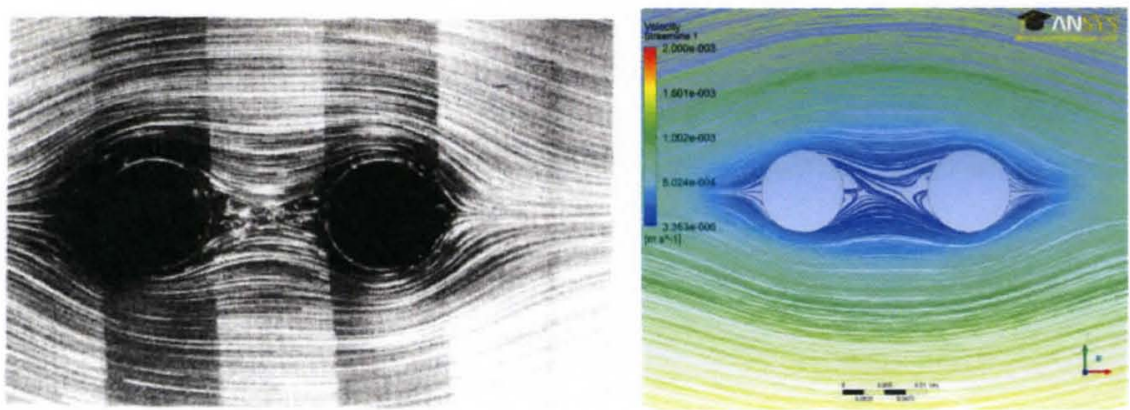

(c)
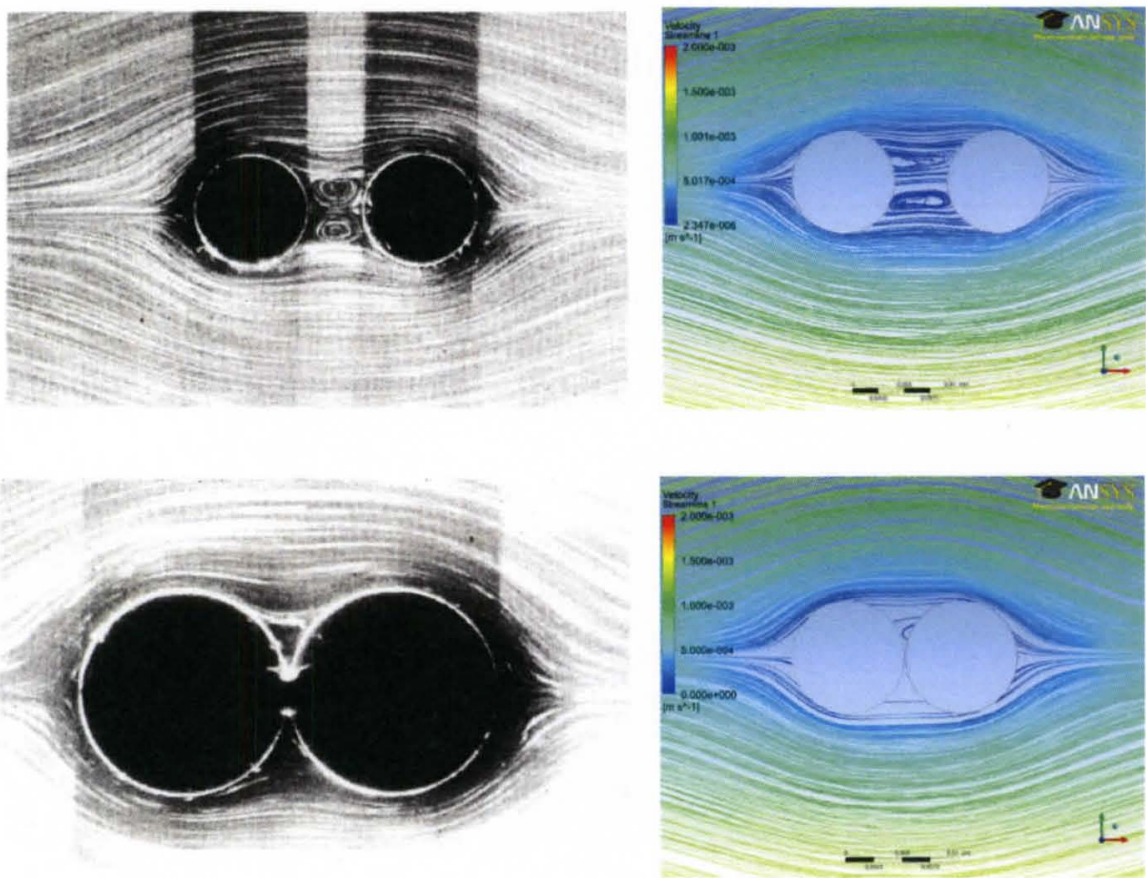

Figure 35. Streamline patterns around two equal circular cylinders:

(a) $\varepsilon / \mathrm{D}=1.5 ;$ (b) $\varepsilon / \mathrm{D}=1.0$; (c) $\varepsilon / \mathrm{D}=0.5$; (d) $\varepsilon / \mathrm{D}=0.0$ 
Another point of validation of the CFX model can be obtained from analytical models based on low Reynolds number flow around a cylinder. Stokes developed a model to determine the force acting on a sphere but was unable to extend this approach to a cylinder. In that case, Stokes noted [47] (as quoted by Lamb[48]):

The pressure of the cylinder on the fluid continually tends to increase the quantity of fluid which it carries with it, while the friction of the fluid at a distance from the cylinder continually tends to diminish it. In the case of a sphere, these two causes eventually counteract each other, and the motion becomes uniform. But in the case of a cylinder, the increase in the quantity of fluid carried continually gains on the decrease due to the friction of the surrounding fluid, and the quantity carried increases indefinitely as the cylinder moves on.

Oseen proposed changes to the Stokes method for the cylinder that successfully resolve this problem; the drag force per unit length of the cylinder can then be calculated using following equation:

$$
F=\frac{4 \pi \mu U}{1 / 2-\gamma-\ln (1 / 2 k a)}
$$

where $\mu$ is dynamic viscosity, $U$ is velocity of flow, $\gamma$ is Euler's constant $(0.577216 \ldots)$, $\mathrm{k}=\mathrm{U} / 2 \mathrm{v}, \mathrm{v}$ is the kinematic viscosity. and $\mathrm{a}$ is the radius of the cylinder.[48] For the current studied problem, the drag force using this equation becomes $0.025 \mathrm{~N} / \mathrm{m}$. The force on each cylinder in the CFX model can be calculated as well; the results as a function of the gap between each cylinder is shown in Figure 36. The figure clearly shows that the drag forces are similar for both the front and rear cylinders, and that they 
approach a constant value as the gap between two cylinders increases (approximately $0.025 \mathrm{~N} / \mathrm{m}$ ). This is reasonable as two cylinders far apart should individually approach the behavior of individual cylinders (i.e. the force value will not change with further separation).

It was anticipated that this force would be in good agreement with the Oseen model; however, the result obtained but the CFX model leads to force values that are roughly 4 times less. One possible explanation for this difference is in the finite element model boundary conditions. The model matches that of the experiment, such that the total width of the tank is $20 \mathrm{D}$. However, the velocity profile shown in Figure 37 indicates that that the boundary is not acting as though it were infinitely far from the cylinders; in that case, the velocity vectors would remain in a straight line near the boundary and they clearly do not. Another possibility is that further mesh refinement is needed. These questions will be investigated using a larger domain with various mesh sizes/densities during the dissertation studies. 


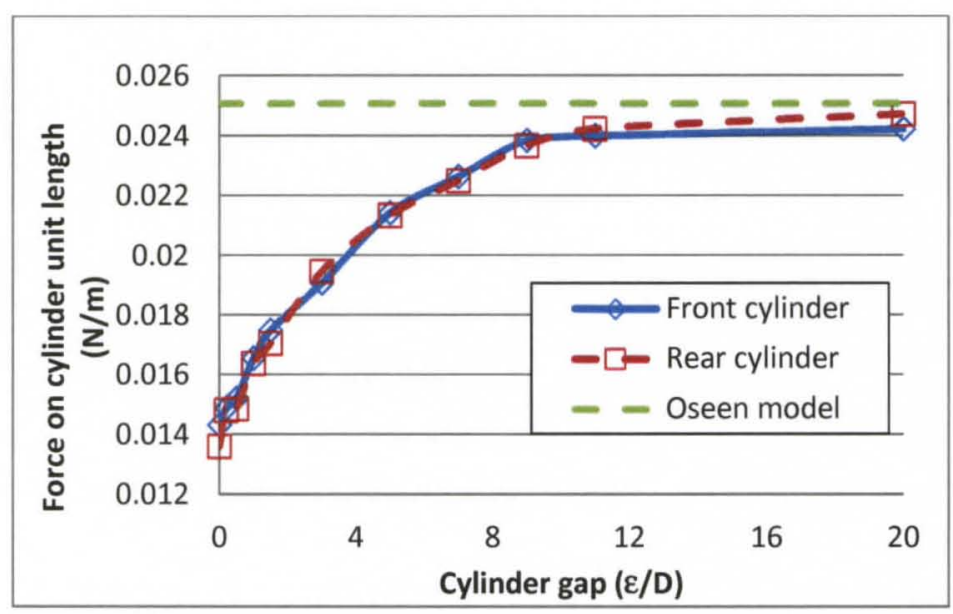

Figure 36. Drag force on each cylinders from CFX simulation compared with the Oseen equation

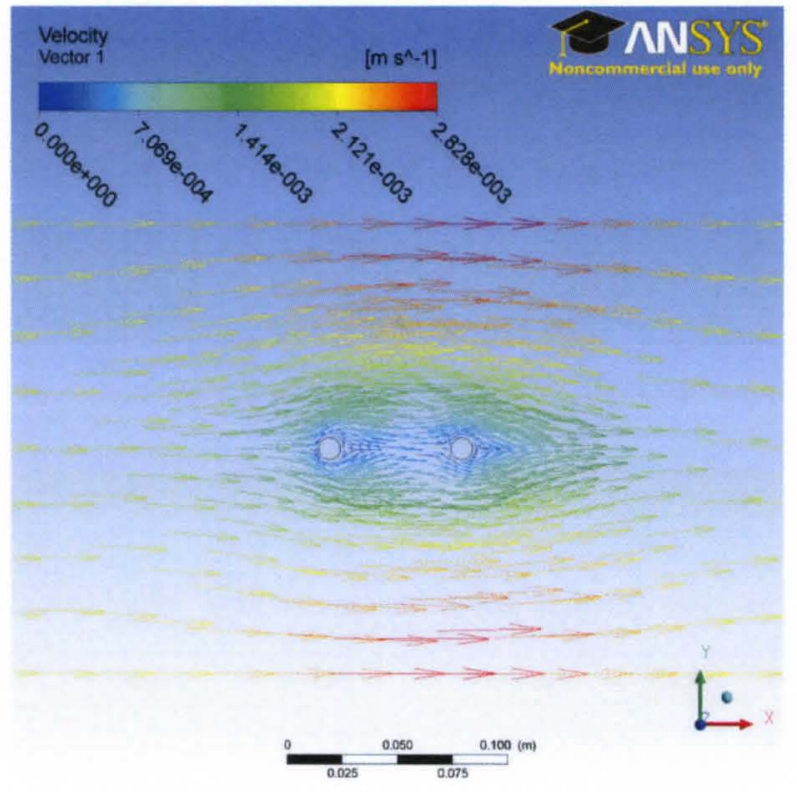

Figure 37 . Velocity vectors for two cylinder model $(\varepsilon / \mathrm{D}=5)$ 


\subsubsection{Results - Cylinder Near Wall Experiment}

Modeling results for fluid flows past a fixed cylinder near the bottom of the fixed

plate are listed below with gap $\varepsilon$ ranges from $0.6 \mathrm{D}, 0.25 \mathrm{D}, 0.1 \mathrm{D}$ and $0 \mathrm{D}$, where $\mathrm{D}$ is the cylinder diameter $(1 \mathrm{~cm})$. The results look very similar to those obtained by the experimental study. 
(a)
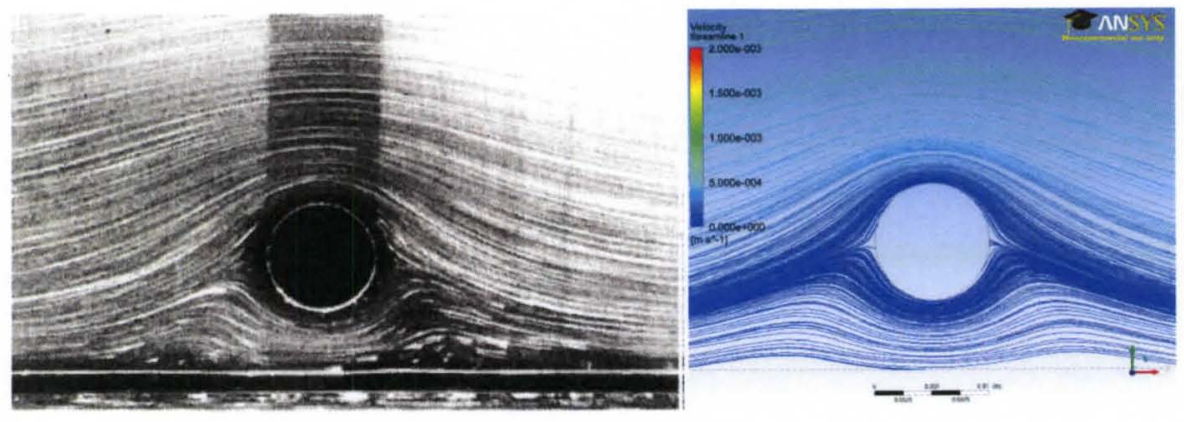

(b)
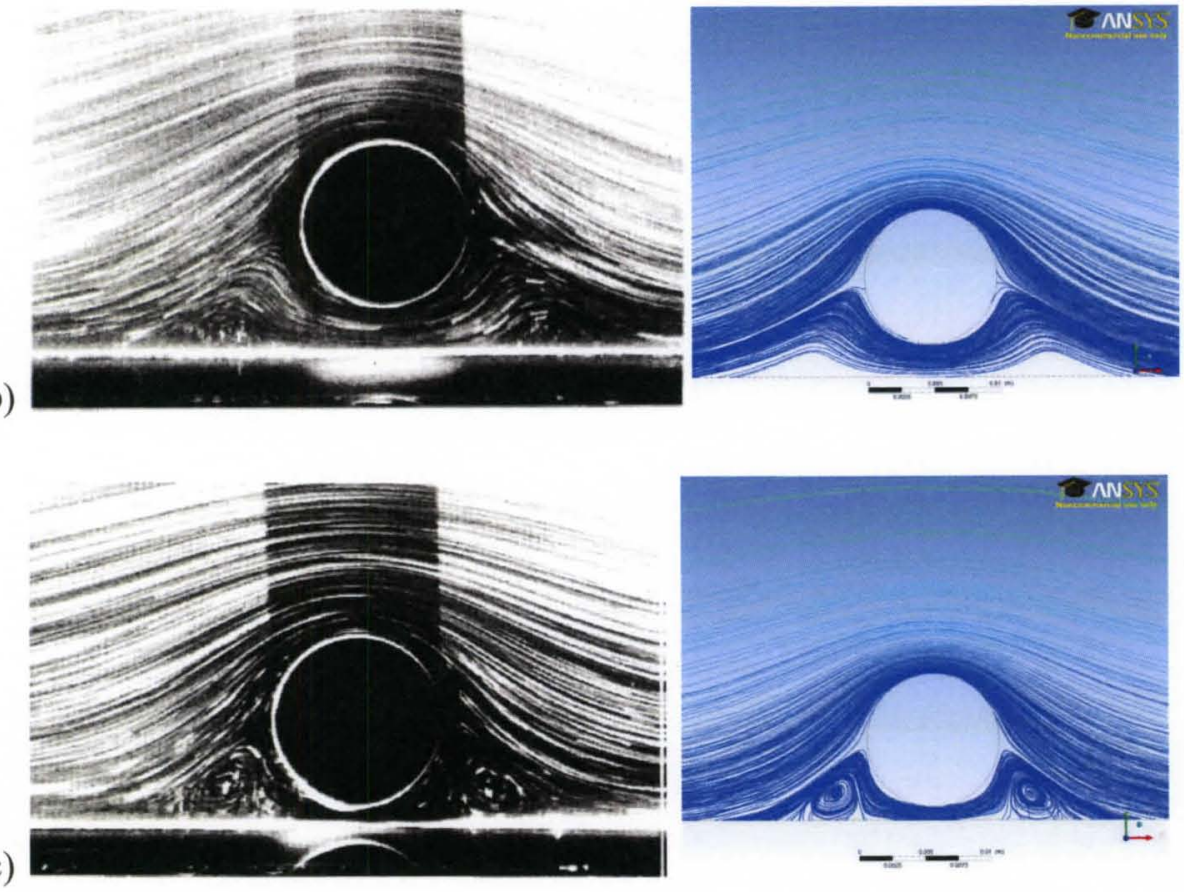

(d)
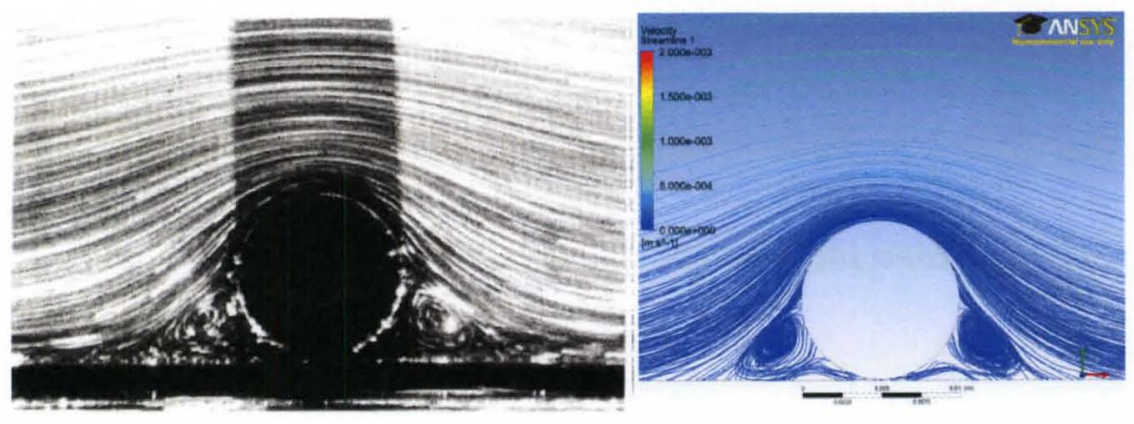

Figure 38. Stream patterns around a circular cylinder placed near a flat plate:

(a) $\varepsilon / \mathrm{D}=1.5 ;$ (b) $\varepsilon / \mathrm{D}=1.0 ;$ (c) $\varepsilon / \mathrm{D}=0.5 ;$ (d) $\varepsilon / \mathrm{D}=0.0$ 


\subsubsection{Summary - Validation of CFX Using Taneda Experiments}

Based on the findings above for two different experiments, it is evident that the approach used with CFX to simulate them leads to comparable results. Differences in terms of the force on the cylinder and the force predicted by the analytical model of

Oseen need to be investigated further. However, it is reasonable to could that CFX can be used as a tool to simulate the phenomena of interest in this proposal, namely the force acting on an oscillating cross-section representing a cantilever beam resonating in a fluid.

\subsection{Studies of 2D Beam Resonating in the Fluid}

The goal of introducing CFD modeling using ANSYS CFX into this project is to evaluate the effect that cross-section shape and the presence of the "flow" handle layer (i.e.) have upon the forces acting on the beam due to a surrounding gas. Past work by $\mathrm{Xu}$ [42] has assumed that the beam can be approximated by an oscillating string of spheres; this approach is identical to that used by Blom et al $[22,42]$ based on the oscillating sphere model in an infinite fluid developed by stokes [49]. In terms of shape, the rectangular cross-section looks closer to a cylinder than a string of spheres. There is also an analytical solution to developed by stokes [49]; it is much more difficult to use compared to the sphere version.

For many cases, the sphere model also can provide a good approximate to the cylinder model as will be demonstrated later. In reality, the cross-section is rectangular in this device and the flow is bounded on the bottom by a flat plane (silicon handle layer) extending in all directions. The use of CFD provides an opportunity to assess the effect of cross-section and the handle layer boundary in terms of fluid forces acting on the beam. 
CFD modeling combines the multiple software which includes SolidWorks for input geometry, ICEM for input mesh file, and ANSYS CFX to define and solve. The case of a cylinder without floor (no presence of the handle layer) is studied first and compared with Stokes' model of an infinitely long cylinder resonating in a fluid. Then three more cases are considered: cylinder with floor; and rectangle without floor; and rectangle with floor. Each of these is compared to the Stokes' model and to each other to evaluate the effect of cross-section shape and the presence of the handle layer.

\subsubsection{Creating Input Geometry}

This creating of the input geometry is done in SolidWorks 2011, and is then used for building the input geometry file for the mesh tool. As an initial study, a model similar to the one studied by Stokes was considered. Since the real beam in this study has a rectanglular cross section with dimensions of $1.1 \mu \mathrm{m} \times 2.0 \mu \mathrm{m}$, the cylinder cases adopted a dimension of diameter of $1.673 \mu \mathrm{m}$, which has an equivalent cross section area with actual beam cross-section. The geometry for the case of a resonating cylinder without floor is shown in Figure 39; in this case a square tank filled with air is interrupted by a cylinder in its center. The dimension of square tank is tested and adjusted to ensure that it is big enough so that no flow effect occurs due to side-walls. This test will be discussed in later section. Based on the geometry of cylinder without floor, the geometry of other three cases could be achieved by changing the shape to rectangular and/or the gap between cylinder/rectangle and the bottom of the tank. The geometry is then saved as ".par" file to use for the next step. 


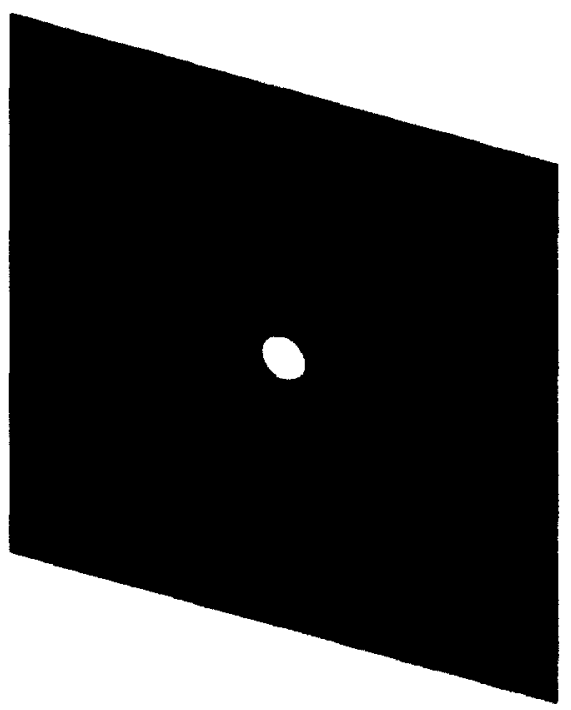

Figure 39. Geometry of cylinder resonating in a fluid

\subsubsection{Creating Input Mesh}

In CFD simulation, mesh size/shape plays a very important role; the quality of the mesh can affect results of the simulation dramatically. ANSYS ICEM CFD is a popular proprietary software package used for CAD and mesh generation. It can create structured, unstructured, multi-block, and hybrid grids with different cell geometries. ICEM CFD is utilized as mesh tool for CFD modeling in this dissertation.

The ".par" geometry file created in SolidWorks 2011 is imported into ICEM CFD by the Workbench Readers menu tool. ICEM creates a structured grid by first saving the geometry as a "tin" file. For the current case, the geometry is very simple so the geometry could also be created in ICEM; however, it is preferable to use Solid works because it is more convenient to change dimensions of geometry in input file. Different parts of the grid are saved under a "part name". Interface (representing beam's cross section), top of tank, bottom of tank and inlet and outlet of tank are defined. This step is 
necessary and important because it related the definition of boundary in conditions and loading CFX.

Once the geometry is created, the next step is to create a block by using the "blocking" tab. A 2D plane block, which is shown below in Figure 40, is then created around the entire geometry and then split up into sections. In Figure 40, the block is combined with 4 squares with dimensions of $1 \mathrm{D}, 2 \mathrm{D}, 10 \mathrm{D}, 40 \mathrm{D}$, and $\mathrm{xD}$ ( $\mathrm{x}$ is the dimension of the tank we choose, D is diameter of cylinder). In each section, the mesh is created by specifying the distribution of points along the edges of the blocks. Therefore, more blocks means more flexibility changing the distribution of element along the edges. The edges and vertices of the blocks must be associated with the geometry curves and points. The section which is more close to the circle gets the more fine mesh for the interface area is what is of greatest interested. The square with the dimension of $1 \mathrm{D}$ will related with the circle which help to make the meshed curve.

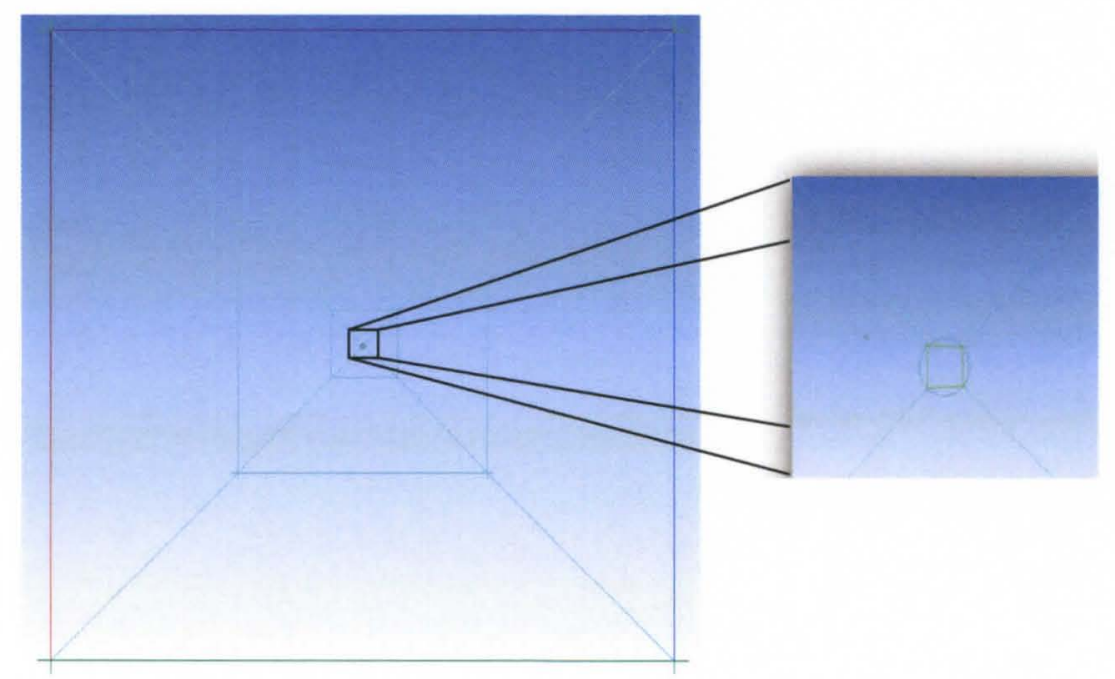

Figure 40. 2D plane block for mesh. 
Once the blocks have been created and all the required points and curves associated, the number of points and the distribution can be set along each edge. In the case studied here, mesh density in the area close to interface should be high to best approximate the gas load, whereas to save time, the sections further should have lower mesh density. There are various types of distribution that can be used. The default set "BigGeometric" is chosen for distribution type. The element size for the different area is listed in the Table 4 for the mesh shown in the Figure 41. We still need to take the test to see if the mesh is fine enough for the calculation which will discuss in the later section. The premesh tool can then be used to view the meshing, and there is also a quality check tool, where we can check if there are any negative, which would suggest that the grid crosses into solid surfaces and will be ejected during CFX solving.

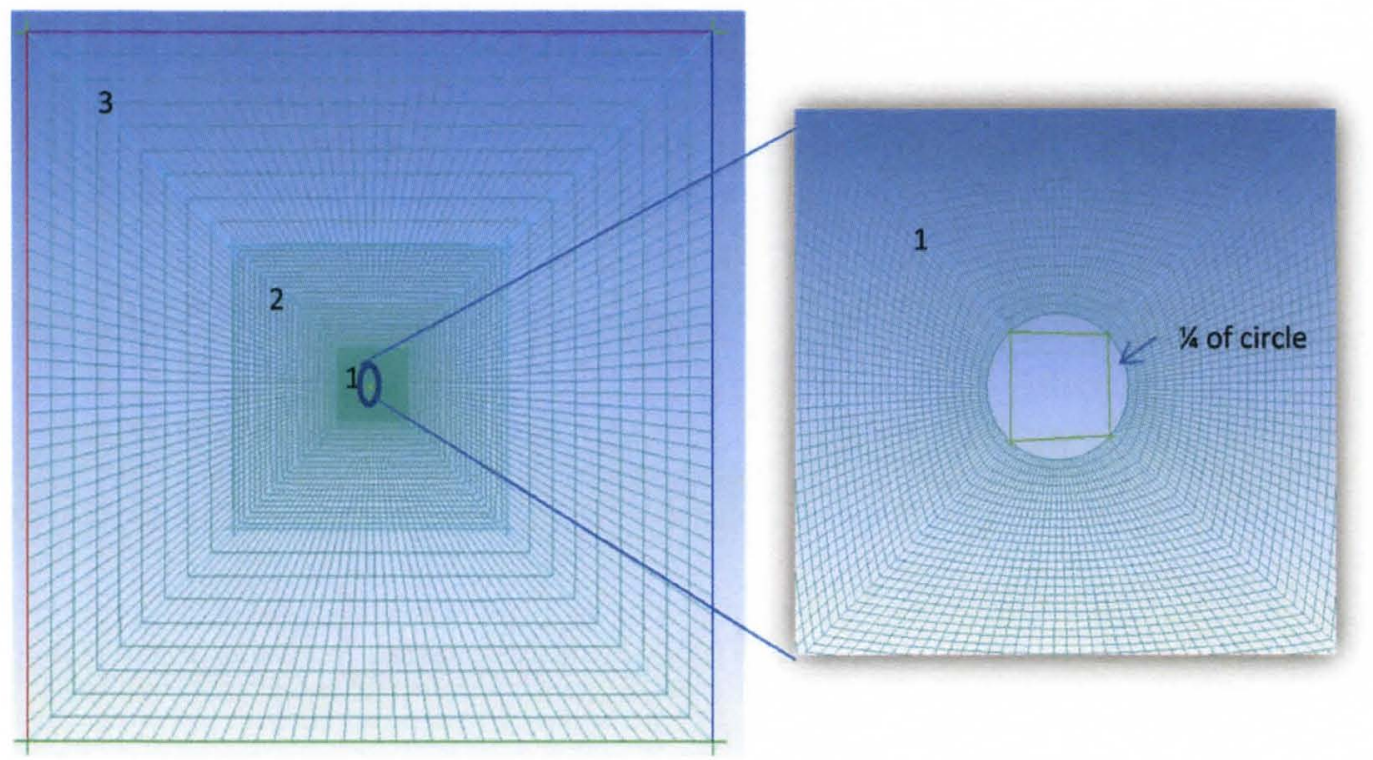

Figure 41. 2D plane block with mesh. 


\begin{tabular}{|c|c|c|c|c|}
\hline Parts & $1 / 4$ of circle & line 1 & line 2 & line 3 \\
\hline Element size $(\mathrm{nm})$ & 66.8 & 145 & 2170 & 9440 \\
\hline Node number & 20 & 100 & 20 & 10 \\
\hline
\end{tabular}

Table 4. The mesh parameters for blocks.

The mesh then was transferred to unstructured mesh and loaded from block, and the 2D mesh file is saved as a 3D "fluent 6 " file by extruding the model a certain distance in the $\mathrm{z}$ direction. By this approach, one element is defined in the $\mathrm{z}$ direction automatically. The "fluent 6 " file could then be imported into CFX and treated similarly as other mesh files. The reason the file is saved as "fluent 6" instead of "CFX 5" is that the "fluent 6 " type will keep all the labels as defined previously use for as the boundary definition in CFX. The "CFX 5" format will extrude the 2D mesh in the $\mathrm{z}$ direction manually; after it is imported into CFX, the block is treated as one part and could not be used to define the boundaries.

\subsubsection{Set Up in CFX-Pre}

The model simulated a cylinder resonating in the air within a container (rectangle box) with a frequency of $40 \mathrm{KHz}$; This value is chosen as it is the reported frequency range for some of the devices tested by Yang [42]. CFX is used to define and specify the simulation settings and physical parameters required to describe the flow problem. The mesh file (*.mesh) created in ICEM CFD with "fluent 6" format is imported into CFX. This input file could not be opened directly. A new CFX-Pre file needs to be created first and imported using the "File->import->mesh". 
Setting up a simulation typically requires defining: materials, domain, simulation type, boundary conditions, and solver control. Air at $25^{\circ} \mathrm{C}$ is chosen from the "material group" list. The material properties are listed in Figure 42. The most important properties to include are density (under Equation of State) and dynamic viscosity (under Transport Properties). In the Outline workspace, "Simulation Type" should be transient simulation for the cylinder resonating in air with the force acting on the cylinder changes with time. For transient simulations, Time Duration and the Timesteps of the simulation have to be specified. Time Duration is a user-specified limit on the length of real time the simulation is to run. Timesteps sets the frequency at which the solver solves the governing equations, (e.g. every $1 \mu \mathrm{s})$.

CFX-Pre generates a default domain automatically. The volume named Fluid in ICEM is chosen in Location and "Fluid Domain" is selected for Domain Type. Select air at $25^{\circ} \mathrm{C}$, non buoyancy, stationary domain motion, and regions of motion specified for mesh deformation from list. For heat transfer, select isothermal type with $25^{\circ} \mathrm{C}$. Because the Reynolds number is very small in this case (about 0.15 the behavior of the flow is treated as laminar. Select "None (Laminar)" option for turbulence function. The initialization tab defines initial conditions for the simulation, with "Automatic with values" setting the initial velocity and static pressure equal to 0 . 


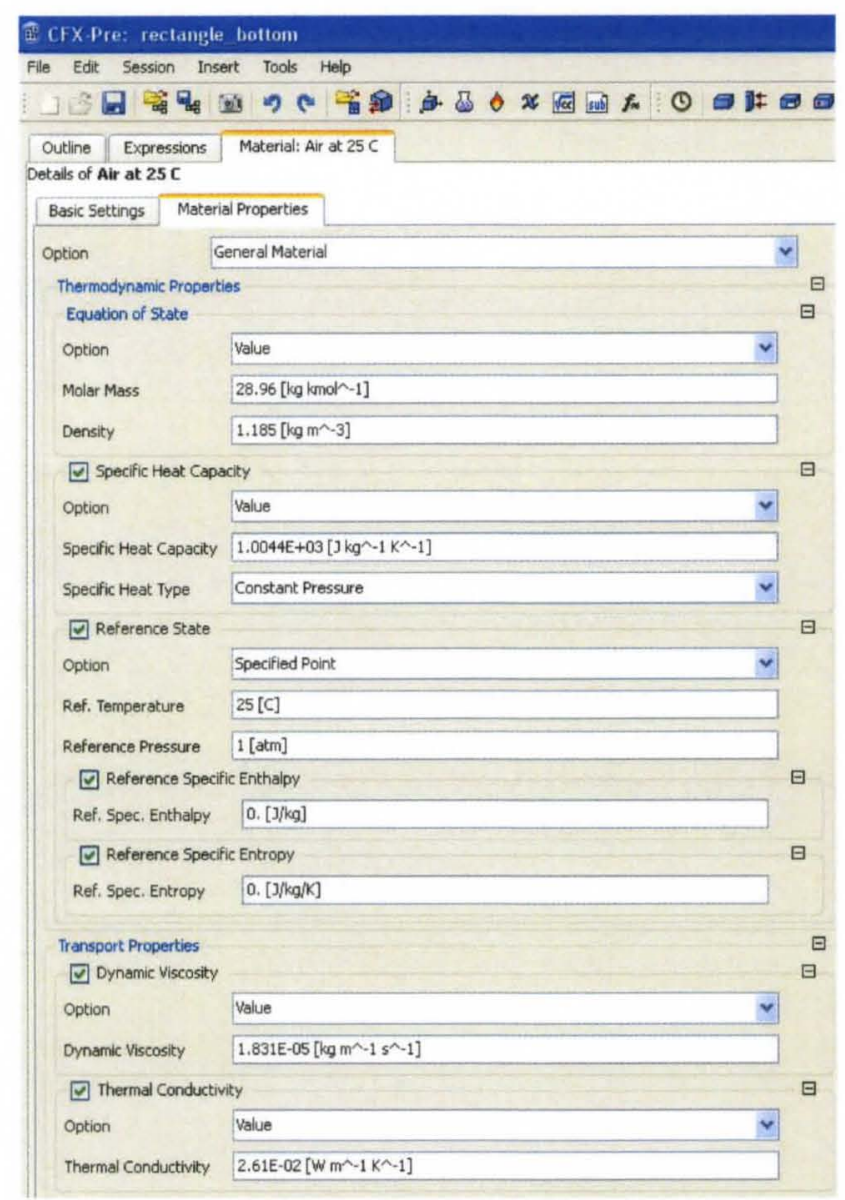

Figure 42. Property parameters set-up for fluid

To create a boundary condition, use the "Create a boundary condition" command and enter a name for the boundary. Boundary conditions are created as interface (beam), inlet, outlet, top wall, bottom wall and symmetry planes.

According to the CFX Help, when the wall velocity is made relative to the boundary frame, only the specified wall velocity is assumed by the flow. When the wall velocity is made relative to the mesh motion, the velocity due to mesh motion is superimposed on the specified wall velocity. In our case, a zero velocity, no slip condition would be applied to all walls. The wall velocity would typically be made relative to the 
mesh motion for the moving cylinder boundary, and relative to the boundary frame for the tank side-walls. This would ensure that the fluid is properly affected by the motion of the cylinder, and that it is not dragged by the motion of the mesh on the tank side-walls.

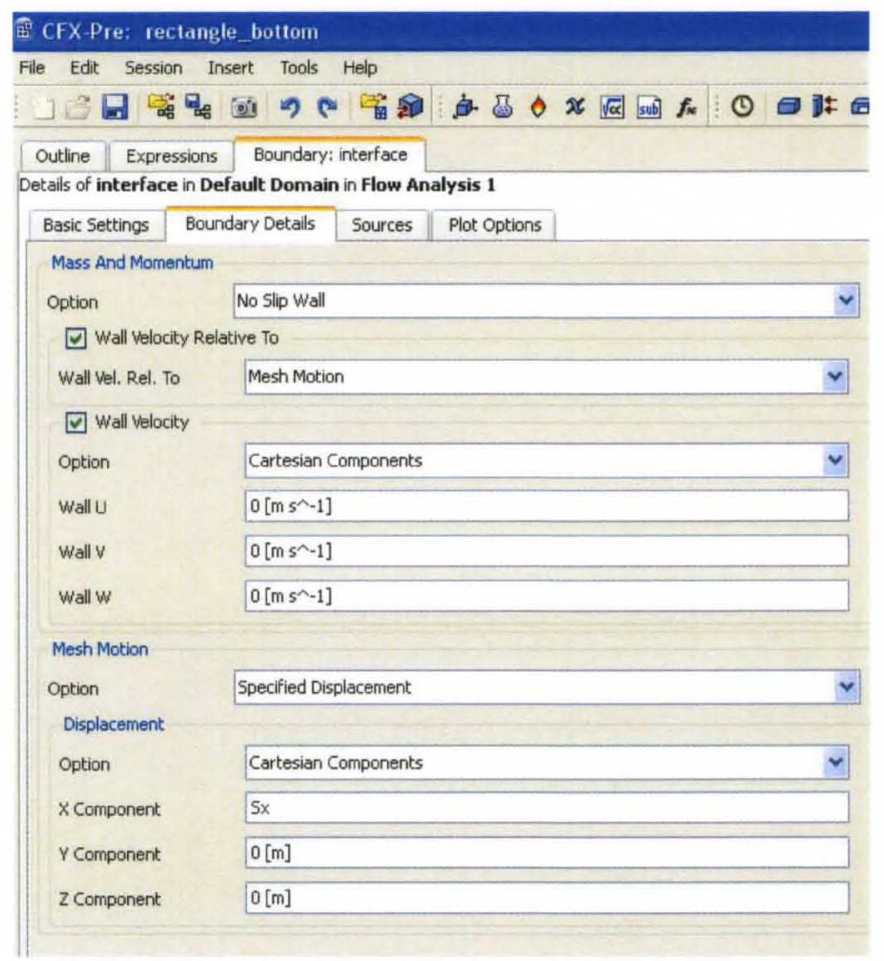

Figure 43. Set-up for interface definition

The set-up for interface is shown in Figure 43. Interface is defined as No Slip Wall with velocity related with mesh motion. The wall velocity for different direction all sets as 0 . Mesh motion is selected specified displacement with $\mathrm{X}$ component equals $\mathrm{Sx}, \mathrm{Y}$ and $\mathrm{Z}$ components set as $0 . \mathrm{S}_{\mathrm{x}}$ is defined in CCL as $\mathrm{S}_{\mathrm{x}}=\mathrm{S}_{0}(\cos (\omega \mathrm{t})-1)$. The reason define the displacement like that is to make the initial velocity equal to 0 .

Figure 44 shows the set-up for tank side-walls. The no slip wall is defined for all walls with velocity relative to boundary frame. The mesh motion for inlet and outlet is 
defined as "stationary", otherwise it is defined as "unspecified" for bottom and top. This will allow for the nodes on the bottom and top surface to move if needed.

Front and back of tank is defined as symmetry plane which makes the 3D problem behave as a $2 \mathrm{D}$ problem to make the computation much faster.
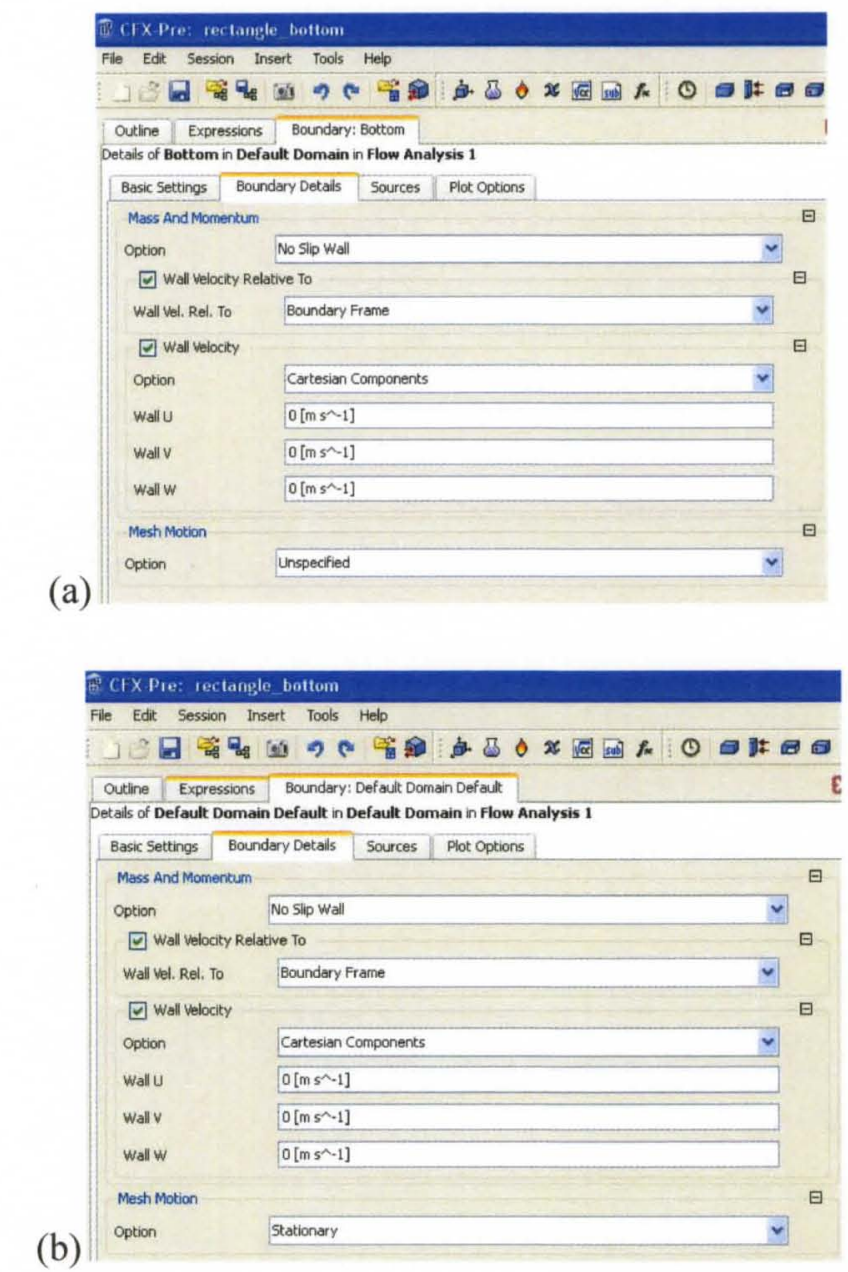

Figure 44 Set-up for tank side-walls definition. (a) Top and bottom; (b) Inlet and outlet.

In solver control, because this case is not very complicated to solve, the default values suffice. In convergence criteria, the most important measure of convergence is the residual. The residual is a measure of the local imbalance of each equation being solved, 
so ideally the residual should decrease as the solution proceeds approaching the final solution. CFX-Solver will terminate each solution step run when the equation residuals calculated are below the residual target value. The Root Mean Square (RMS) type of residual is used, with the default RMS target of 0.0001 used. For transient simulations, the Solver solves the governing equations at regular time intervals. To achieve convergence at each time step, a number of loop iterations have to be performed. Once convergence has been achieved at one time step, or the maximum number of loops is reached, the solver proceeds to the next time step, and repeats the process.

Output control panel is used to manage the way files are written by the solver. The transient results are written at specified time intervals in addition to the full results file. When the simulation set-up is complete, a definition file needs to be written which defines all the simulation using the "Write Solver Input File" icon from the toolbar.

\subsubsection{Patch Test}

The patch test in the finite element method is a simple indicator of the quality of a finite element model. The patch test uses a partial differential equation on a domain consisting from several elements set up so that the exact solution is known. Typically in mechanics, this prescribes the exact solution as displacements that vary as linear function in space (called a constant strain solution). The elements pass the patch test if the finite element solution is the same as the exact solution. It was long conjectured that passing that patch test is sufficient for the convergence of the finite element; that is, to ensure that the solutions from the finite element method converge to the exact solution of the partial differential equation as the finite element mesh is refined. However, this is not the case, and the patch test is neither sufficient nor necessary for convergence.[50] 
The same idea as the patch test is used here to determine the proper set-up in modeling. This section will test and discuss several possible factors (dimension of the tank boundaries, element size along the cylinder, refine level, the transient timesteps and the maximum mesh movement $S_{0}$ ) that might play a role in the simulation. The force acting in the cylinder surface in $\mathrm{x}$ direction is used as a validity check to see whether the set-up is enough for convergence. This force is calculated by the CFX function equation "force_x@interface". To get the force per unit length, it needs to be divided by the model depth in the $z$ direction.

\subsubsection{Dimension of the boundaries}

The outer box surface is defined as no slip wall. The dimension should be in a proper scale because (a) if it is too small, the simulation results will be dramatically affected by the wall, (b) but if it is too big, it will lead to longer simulation time with little benefit or improvement in solution. The first goal is to identify a proper dimension. For this goal, different length on the edges of the rectangle box was tried as 50D, 100D, 150D and $200 \mathrm{D}$ where $\mathrm{D}$ is the diameter of the cylinder.

In ICEM CFD, a block is used to divide the model into 3 regions. The first one is the critical area closest to the cylinder (inside the dark green square of Figure 2) and is defined as a square with edge of $10 \mathrm{D}$. The second one is next to the first one and defined as a square with edge of 40D. The rest outside of the second region is the third one and it is the size that is varied. The mesh for the first region and the second region stays exactly the same no matter what the outer box size is. The third region is meshed by the same element length in all cases. Two sample blocks are shown in Figure 45 with dimensions 
of $100 \mathrm{D}$ and $150 \mathrm{D}$. The nodes chosen along the dialog (half) of the square and element size chosen for different region are listed in Table 5.

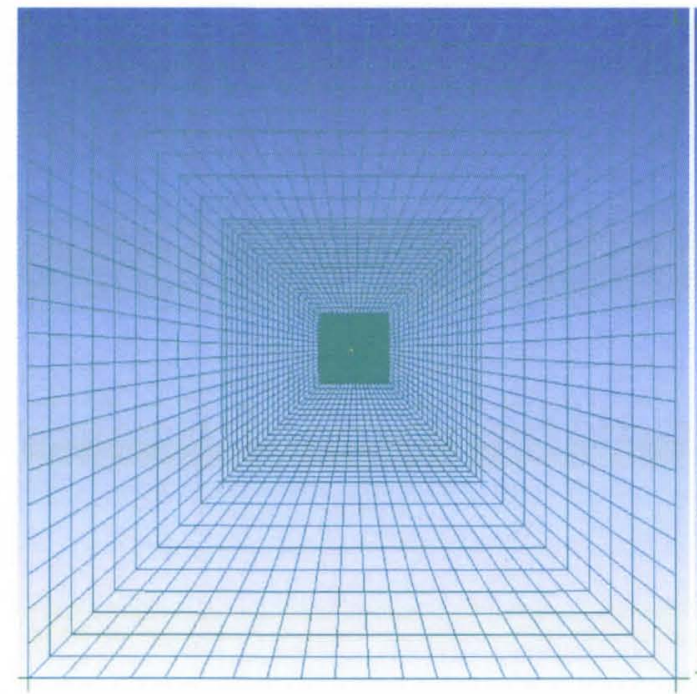

(a)

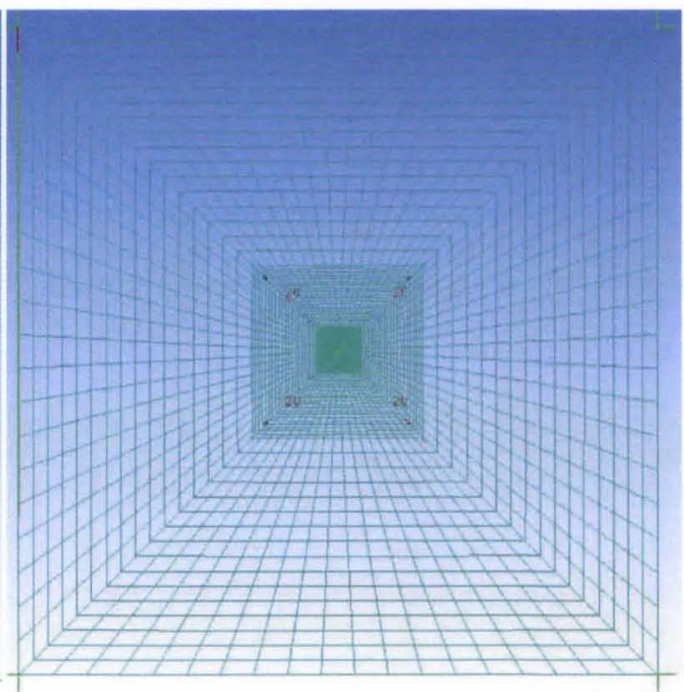

(b)

Figure 45. Block of mesh: (a) 100D; (b) 150D

\begin{tabular}{|l|c|c|}
\hline & Node count per edge & Element Length(nm) \\
\hline Part 1 & 100 & 145 \\
\hline Part 2 & 20 & 2170 \\
\hline Part 3 & varies & 9440 \\
\hline
\end{tabular}

Table 5. Nodes number and element length along the dialog of square

The maximum mesh movement $\mathrm{S}_{0}$ of $0.2 \mu \mathrm{m}$, "timesteps" of $1 \mu \mathrm{s}$, and "total time" of 10 cycles are defined for resonating in CFX-Pre. The results of modeling set-up discussed above are displayed in Figure 46. This shows force acting at the interface in the $\mathrm{x}$ direction convergent to the value about $4.4 \mu \mathrm{N} / \mathrm{m}$ when the dimension of tank boundary is $100 \mathrm{D}$ or greater. It is clear that $100 \mathrm{D}$ is big enough to avoid the wall effect. A small 
size of 50D was also tested, but time step of $1 \mu$ s is too big for 50D and results are not reliable and are listed in Figure 46.

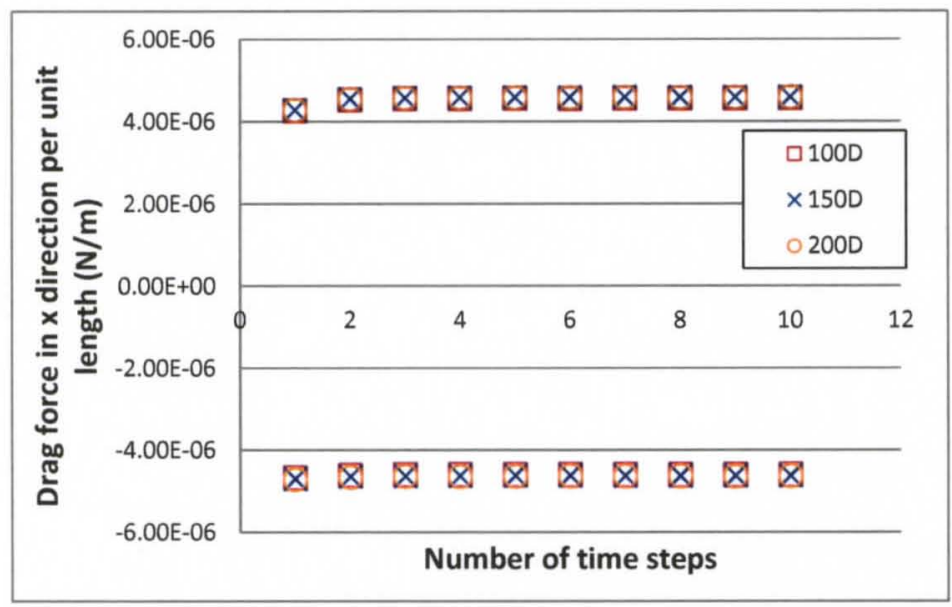

Figure 46. Maximum drag force of each cycle in cylinder surface according to different boundary dimension

\subsubsection{Element number along the cylinder}

The edge of the cylinder is divided into 4 parts by the block and each one parallels to the related square edges of block (as shown in Figure 41). Each edge could be meshed using different element number. Figure 47 shows 40 elements and 60 elements on each edge. Element counts of 20, 40, 60, 80, and 100 on each quarter of the interface were tested to check if this could be a factor that affects the simulation results. The dimension of tank boundaries is set as $100 \mathrm{D}$, which was already determined in the previous section. The other were repeated same for this test. Calculation results show that when the edge is divided in more than 60 elements, the "timesteps" of $1 \mu$ s is not small enough to get the expected results. According to this phenomenon, "timesteps" was changed to a smaller 
one and after several different values were tried, "timesteps" of $0.1 \mu$ s is chosen to do the comparison.

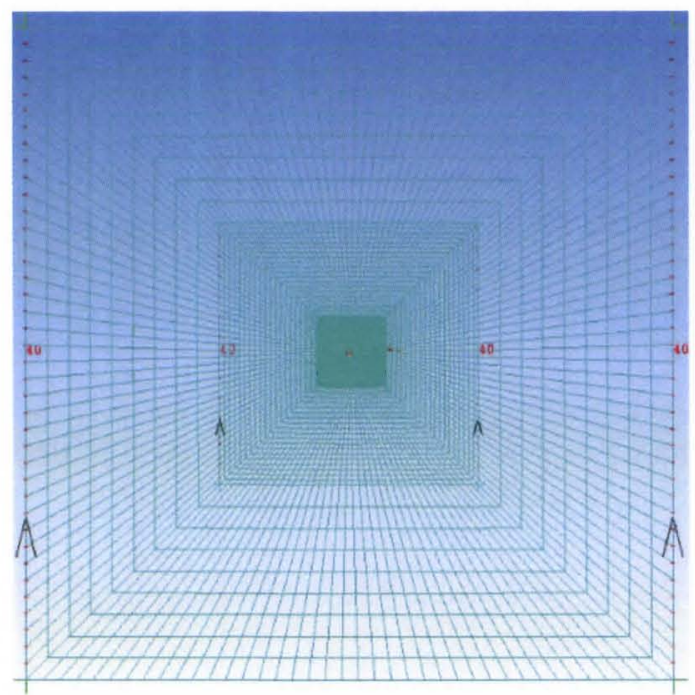

(a)

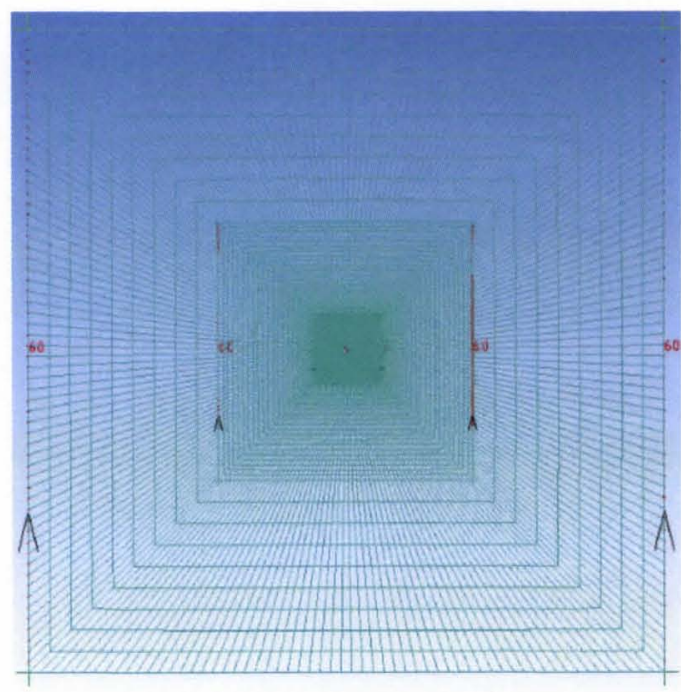

(b)

Figure 47. Mesh block of different element number along edge. (a) 40; (b) 60 .

As shown in Figure 48, the drag force in the cylinder becomes smaller when increasing the number of elements. However this change is relatively small. Table 6 listed mesh element number and related calculation time for the different set-up. It is obvious that the more elements divide the edge, the more elements get produced in the model. Thus time for calculation increase significantly when more elements get involved in the model. What need to be addressed is to find the balance of quality and efficiency. For timestep equals $1 \mathrm{e}-7 \mathrm{~s}$, the 20 elements or 40 along the rectangle edge are a good setup to achieve some fine results. 


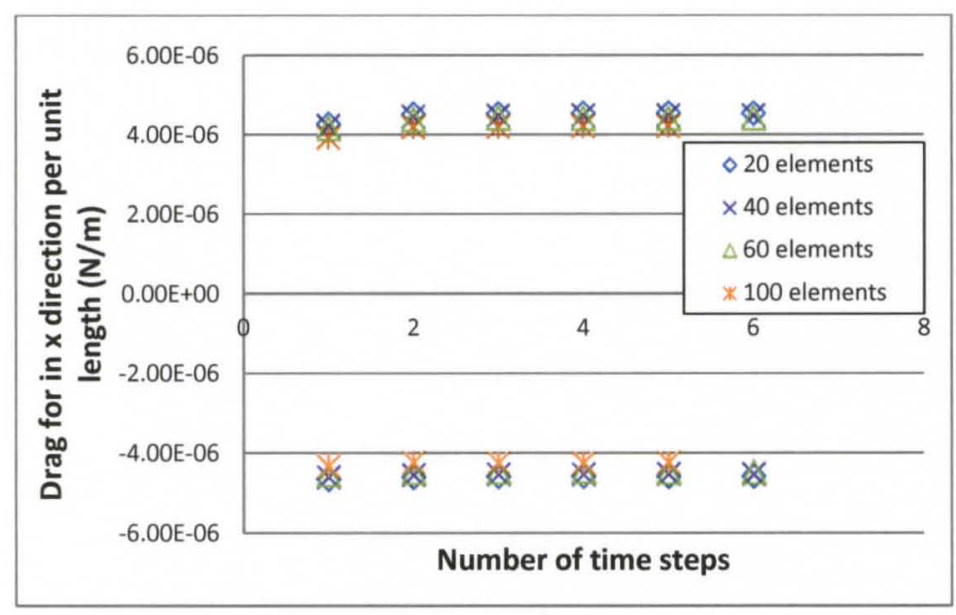

Figure 48. Maximum drag force for each cycle in the cylinder surface according to the different element number along the boundary edge.

\begin{tabular}{|c|c|c|c|c|c|c|}
\hline & $\begin{array}{c}\text { Timestep } \\
(\mu \mathrm{s})\end{array}$ & $\begin{array}{c}\text { Number of } \\
\text { cycles }\end{array}$ & Number of division & Refine level & $\begin{array}{l}\text { Number of } \\
\text { elements }\end{array}$ & Calculation time (s) \\
\hline \multirow{5}{*}{ 100D } & \multirow{3}{*}{0.10} & \multirow{3}{*}{6} & 20 elements & & 19304 & $5.13 E+03$ \\
\hline & & & 40 elements & & 39624 & $8.99 \mathrm{E}+03$ \\
\hline & & & 60 elements & & 59944 & $1.06 \mathrm{E}+04$ \\
\hline & \multirow{2}{*}{1.00} & 6 & 20 elements & level 2 & 77216 & $5.69 \mathrm{E}+03$ \\
\hline & & 10 & 20 elements & level 1 & 19304 & $3.32 \mathrm{E}+03$ \\
\hline 150D & 1.00 & 10 & 20 elements & & 20368 & $3.48 \mathrm{E}+03$ \\
\hline 200D & 1.00 & 10 & 20 elements & & 21584 & $2.46 \mathrm{E}+03$ \\
\hline
\end{tabular}

Table 6. Summary of mesh element number and calculation time

\subsubsection{Refine level}

In ICEM CFD, there are refine functions available to refine the mesh. The refine level 1 and 2 are tried for comparison which is shown in Figure 49. Table 6 demonstrated the mesh elements and calculation time both get increased (almost doubled), while the 
simulation results in Figure 50 demonstrated that the result is convergent using level 1; therefore it is assumed that refine level 1 is good enough to be used for calculation.

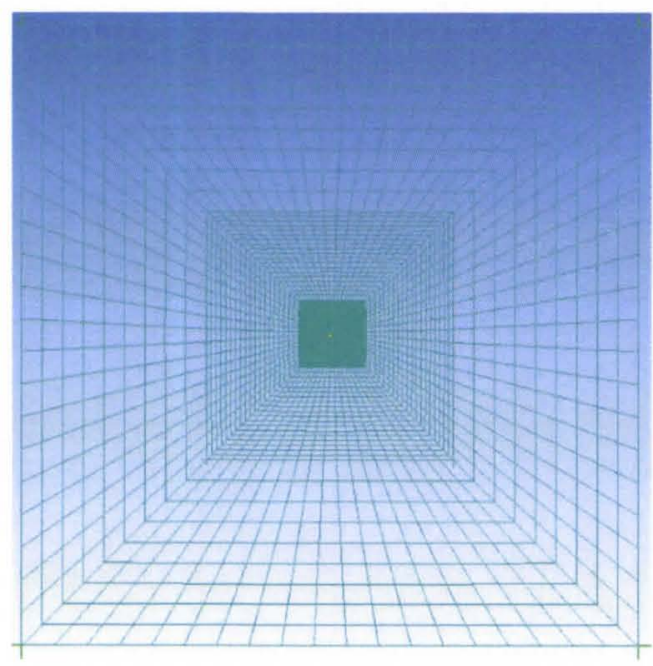

(a)

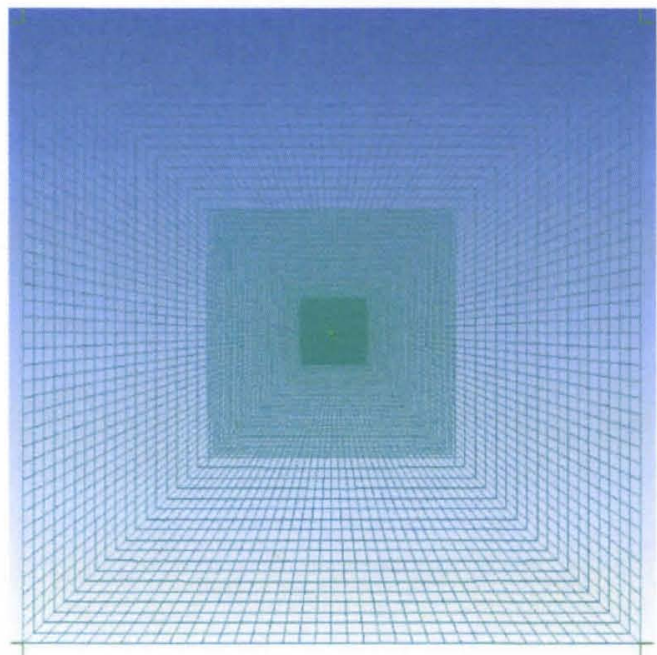

(b)

Figure 49. Mesh block of different refine level. (a) level 1; (b) level 2

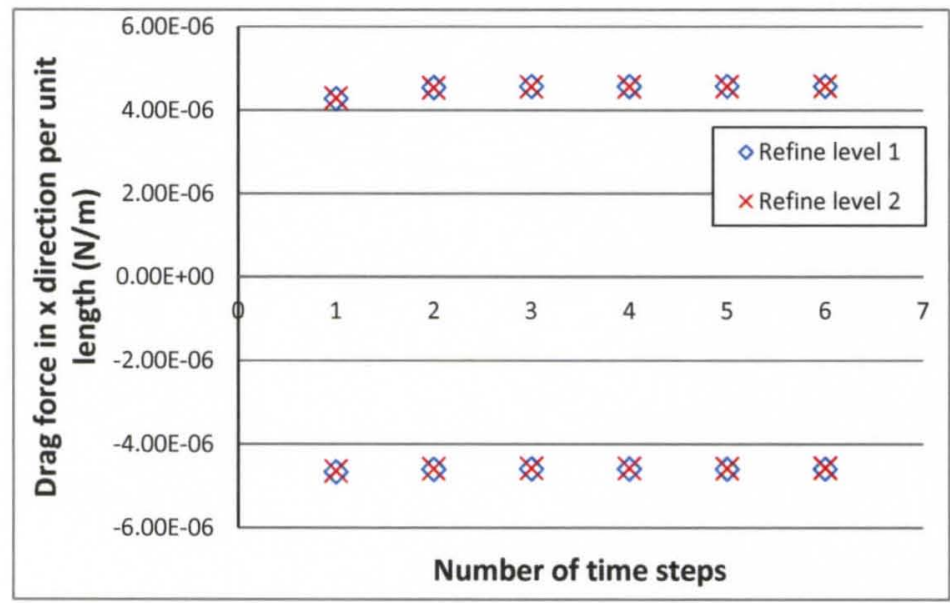

Figure 50. Maximum drag force for each cycle in cylinder surface according to different refine levels 


\subsubsection{Time step}

Time dependent behavior for transient simulations in ANSYS CFX is specified through "Time Duration" and "Timesteps". The "Timesteps" option provides a way for ANSYS CFX to track the progress of real time during the simulation. During simulation, not all "Timesteps" values were proper for calculation. For example, "timestep=0.1 $\mu \mathrm{s}$ ", and "timestep $=0.25 \mu \mathrm{s}$ " set-ups for boundary dimension of $50 \mathrm{D}$ with 20 elements will lead to very different results of drag force as shown in Figure 51.
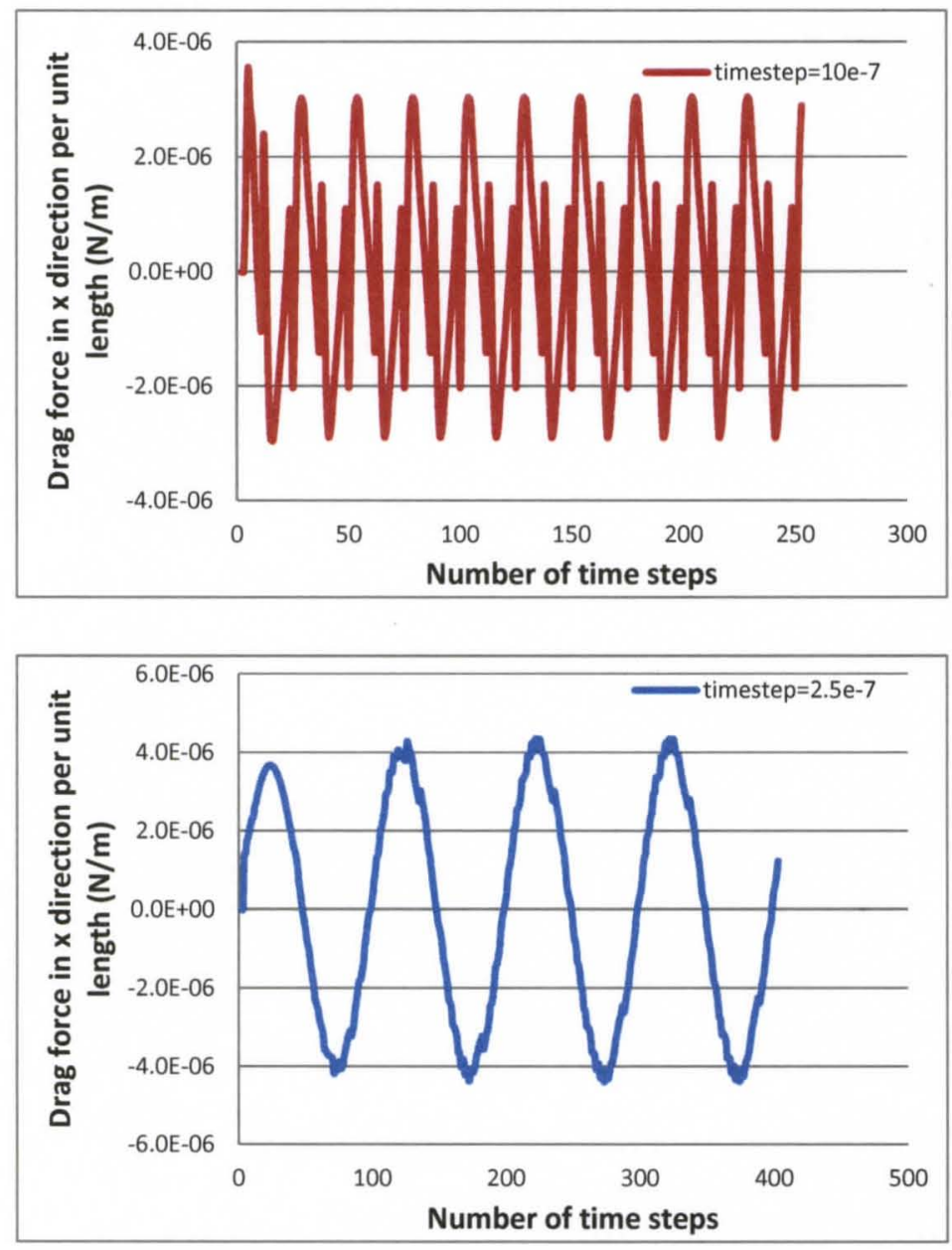

Figure 51. Drag force in the cylinder surface according to the different timestep set-up. 
According the choice in boundary dimension, 100D of 20 elements are tried for different "Timesteps" set-up. The simulation results are shown in Figure 52 which shows that the "Timesteps" of $0.25 \mu$ s is only slightly different from others. "Timesteps" of 0.1 $\mu \mathrm{s}$ and $0.1 \mu \mathrm{s}$ is pretty close. Considering significant increase of calculation time, "Timesteps" of $1 \mu \mathrm{s}$ is chosen as the best choice to provide both an accurate solution in the least time.

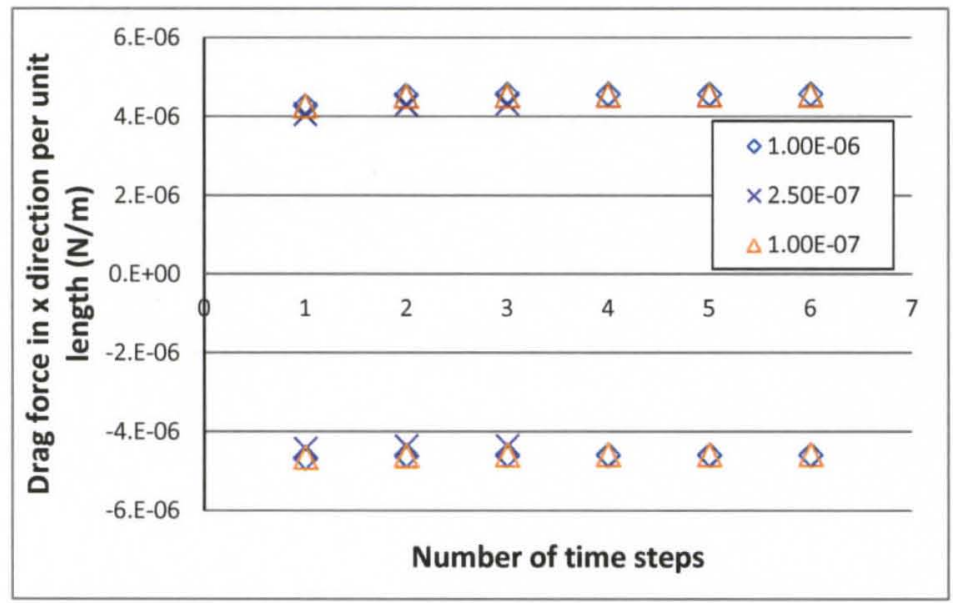

Figure 52. Maximum Drag force for each cycle in the cylinder surface according to the different timesteps in calculation.

The drag force acting at the cylinder surface in $\mathrm{x}$ direction for set up of "Timesteps" ="10 e-7" is shown in Figure 53 (a) which changes with time. The shape of drag force is not exactly sinusoidal because the time step is too big to capture the small change in detail. After time step switched to 1e-7, the result changed dramatically and the drag force changed sinusoidal with time as shown in Figure 53 (b). If the accurate change detail of drag force is required, the "timestep" should be 1e-7 (or less); this certainly will take more time to calculate than a large time step size. 

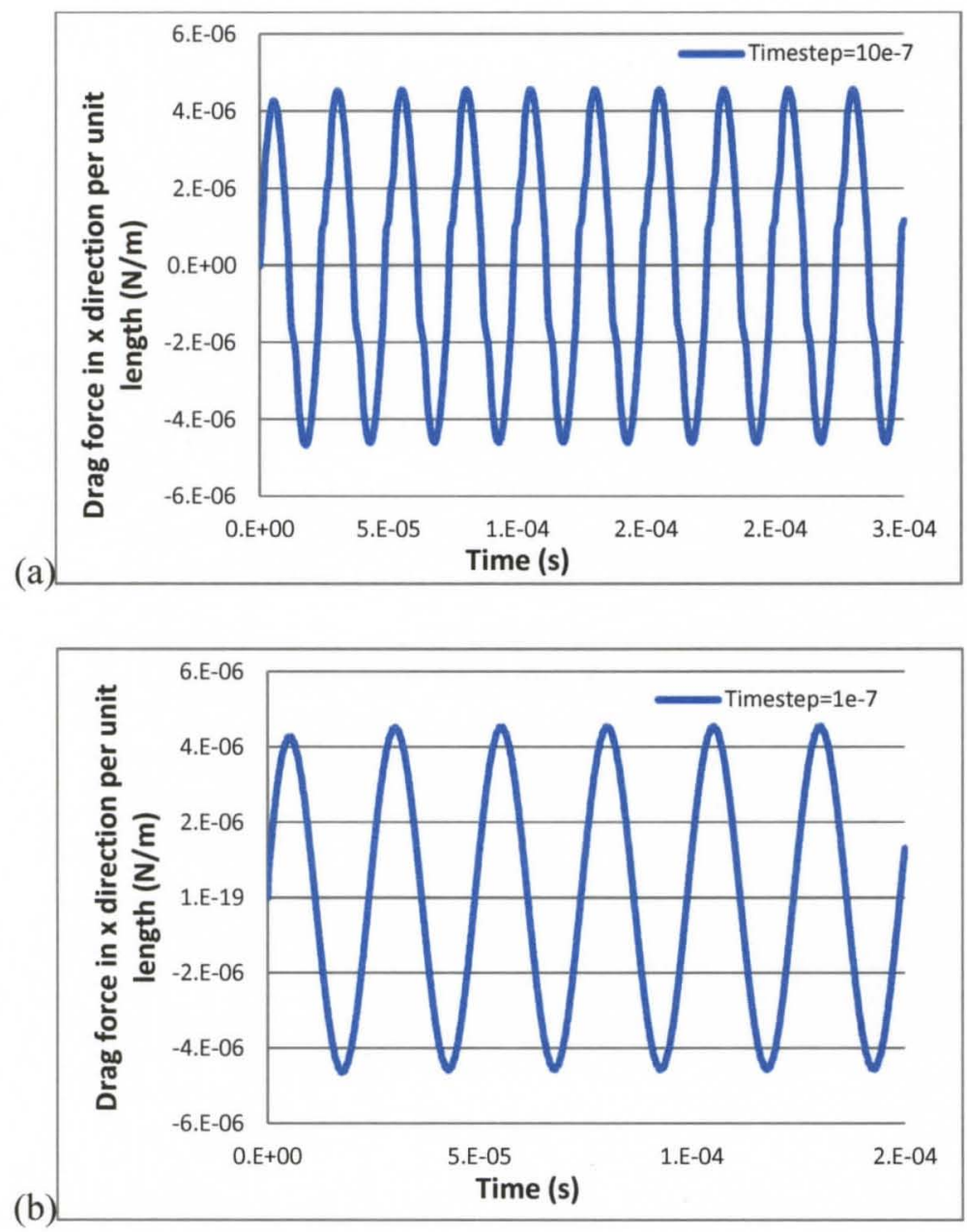

Figure 53. Drag force in $\mathrm{x}$ direction per unit length versus with time for $\mathrm{S}_{0}=0.2 \mu \mathrm{m}$.

(a) time step=10E-7; (b) time step=1E-7.

\subsubsection{Maximum mesh cylinder movement}

Maximum mesh cylinder movement, $\mathrm{S}_{0}$, was also checked to see how it affects the simulation. According to Stokes's model, the force should scale linearly with velocity and accelerate (Equation (6)). $\mathrm{S}_{0}$ of $2 \mu \mathrm{m}, 0.2 \mu \mathrm{m}$ and $0.02 \mu \mathrm{m}$ were chosen for trial. The results show that the peak drag force changes linearly with $\mathrm{S}_{0}$; this shows that $\mathrm{S}_{0}$ equal to $0.2 \mu \mathrm{m}$ is a reasonable value for solution. 


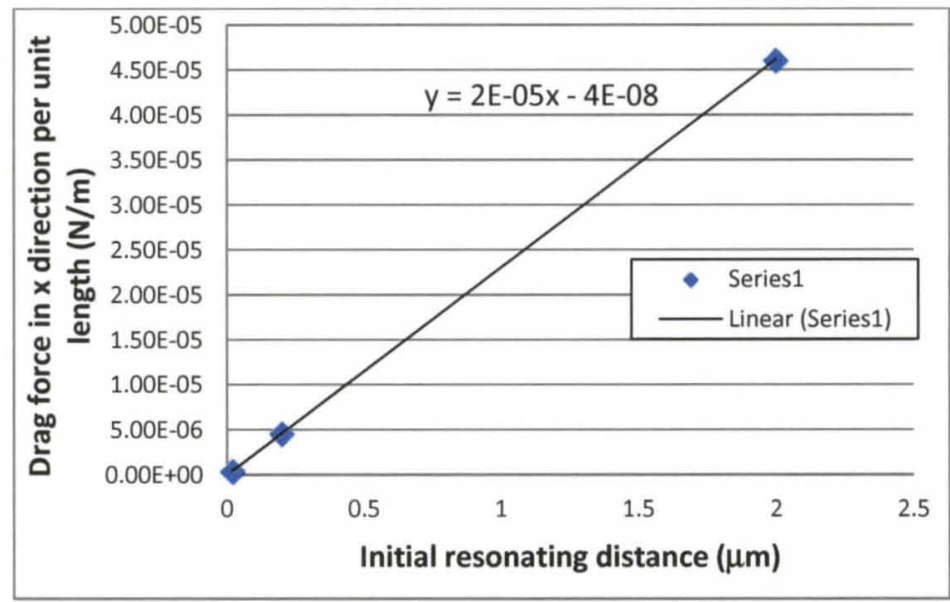

Figure 54. Drag force in the cylinder surface according to the different initial resonating distance.

To summarize the findings in this simulation study, the mesh and calculation setup with a boundary dimension of 100D, 20 elements along the edge of the boundary, refine level 1, and "Timesteps" of $1 \mu \mathrm{s}$, and $\mathrm{S}_{0}$ of $0.2 \mu \mathrm{m}$ are proper for simulating the cylinder resonating in the air when surrounded by a square container. The maximum force acting at interface in $\mathrm{x}$ direction per unit length converges to the value of 4.58 $\mu \mathrm{N} / \mathrm{m}$. The force acting at interface in $\mathrm{x}$ direction per unit length changes with time, and shows in Figure 53 (a). For boundary dimension of 150D, 20 elements along the edge of the boundary, refine level 1, "Timestep" of $10 \mathrm{e}-7$ and $\mathrm{S}_{0}$ of 0.2 , the drag maximum drag force per unit length is $4.61 \mu \mathrm{N} / \mathrm{m}$. We will compare these values to the Stokes theory.

\subsection{Comparison with Stokes Model}

In previous research, Yang cited the model by Blom et al for the damping analysis of the microcantilever beams. As noted earlier, the assumption was made that the beam could be considered as a string of spheres. If the spheres are assumed to vibrate 
independently of each other, each with infinite separation from its neighbors, the resulting drag force per unit length is the drag force on a single sphere divided by the distance between each sphere.

This approach is useful as there is an existing analytical model derived by Stokes and presented in the form below by Lamb. In this case, the sphere is assumed to be a ball that is oscillating on a pendulum in an infinite mass of fluid. The pendulum motion is assumed to be small, which causes the motion be oscillatory in a single direction; any effects of the pendulum cable are neglected, the fluid is assumed to be incompressible, and the flow is such that the Reynolds number is low. The force on the sphere is periodic and has the form:

$$
\begin{gathered}
F_{\text {sphere }}=-m_{s} k \frac{d U}{d t}-m_{s} k^{\prime} \omega U \\
k=\frac{1}{2}+\frac{9}{4 \beta R} \quad ; \quad k^{\prime}=\frac{9}{4 \beta R}\left(1+\frac{1}{\beta R}\right) ; m_{s}=\frac{4}{3} \pi \rho R^{3} \quad ; \quad \beta=\sqrt{\frac{\omega}{2 v}}
\end{gathered}
$$

where $U$ and $d U / d t$ are the velocity and acceleration of the sphere, respectively, $\rho$ and $v$ are the density and dynamic viscosity of the fluid, respectively, $\mathrm{R}$ is the radius of the sphere, $m_{s}$ is the mass of the fluid displaced by the sphere, $\omega$ is the frequency of oscillation. The first term is used to provide an additive mass for the actual sphere, representing the effective mass of fluid that is accelerated with the sphere during oscillation. The second term provides a damping force due to fluid viscous forces acting on the sphere. Blom et al ultimately neglected the inertia term, based on the argument that the effective mass of the gas is much smaller than the mass of the vibrating beam. This model was shown to match experimental beam resonance data by both Blom et al 
and $\mathrm{Xu}$ when a suitable value of $\mathrm{R}$ is chosen (a fitting parameter). Yang also reported that a single value of $\mathrm{R}$ once shosen predicted experimental findings for a number of gases and pressure.[42]

Stokes also pursued a similar model for an infinitely long cylinder on a pendulum; this becomes a 2D model which is solved by an infinite series approach. Lamb notes that this approach is analogous to using complex polar coordinates with Bessel functions having argument $(1-$ i) $\beta R .[48]$ The solution for the force per unit length acting on the cylinder takes a similar form as above:

$$
F_{c y l i n d e r}=-m_{c} k \frac{d U}{d t}-m_{c} k^{\prime} \omega U \quad ; \quad m_{c}=\pi \rho R^{2}
$$

where $R$ is the cylinder radius, $\rho$ is the fluid density, and $m_{c}$ is the mass of the gas displaced by the cylinder (per unit length). Unlike the sphere case, however, the terms of $\mathrm{k}$ and $\mathrm{k}$ ' cannot be expressed by a compact algebraic form in terms of $\beta \mathrm{R}$. Instead, these take the form of 8 complex infinite series. These are combined in a particular ratio, with some in the numerator and some in the denominator; the result is then evaluated,with $\mathrm{k}$ and k' becoming the real and imaginary parts, respectively. These are tabulated by Stokes; the result is shown in Table 7 in terms of the parameter $m=1 / 2 R(\omega / v)^{1 / 2}$. 


\begin{tabular}{|c|c|c|c|c|c|c|c|c|}
\hline $\mathbf{m}$ & $k^{\prime}$ & $\mathbf{m}^{2} k$ & $\mathbf{m}^{2} k^{\prime}$ & $\mathbf{m}$ & $k$ & $k^{\prime}$ & $\mathbf{m}^{2} k$ & $m^{2} k^{\prime}$ \\
\hline 0 & $x$ & 0 & 0 & 2.1 & 1.677 & .7822 & 7.395 & 3.450 \\
\hline$-1 \mid 19.70$ & 48.63 & .1970 & .4863 & 2.2 & $1.6+6$ & .7421 & 7.966 & 3.592 \\
\hline .29 .166 & 16.73 & .3666 & .6691 & 2.3 & 1.618 & .7059 & 8.557 & 3.734 \\
\hline .36 .166 & 9.258 & .5549 & .8832 & 2.4 & 1.592 & .6730 & 9.168 & 3.877 \\
\hline+4.771 & 6.185 & .7633 & .9896 & 2.5 & 1.568 & .6430 & 9.799 & 4.019 \\
\hline .53 .968 & 4.567 & .9920 & 1.142 & 2.6 & 1.546 & .6154 & 10.45 & 4.160 \\
\hline 63.445 & 3.589 & 1.240 & 1.292 & 2.7 & 1.526 & .5902 & 11.12 & 4.303 \\
\hline-73.082 & 2.936 & 1.510 & 1.439 & 2.8 & 1.507 & .5669 & 11.81 & 4.444 \\
\hline 82.812 & 2.477 & 1.800 & 1.585 & 2.9 & 1.489 & .5453 & 12.52 & 4.586 \\
\hline .92 .604 & 2.137 & 2.110 & 1.731 & 3.0 & 1.473 & .5253 & 13.25 & 4.728 \\
\hline 1.02 .439 & 1.876 & 2.439 & 1.876 & 3.1 & 1.457 & .5068 & 14.01 & 4.870 \\
\hline $1.1 \mid 2.306$ & 1.678 & 2.790 & 2.021 & 3.2 & 1.443 & .4895 & 14.78 & 5.012 \\
\hline 1.22 .194 & 1.503 & 3.160 & 2.164 & 3.3 & 1.430 & .4732 & 15.57 & 5.154 \\
\hline 1.32 .102 & 1.365 & 3.552 & 2.307 & 3.4 & 1.417 & .4581 & 16.38 & 5.296 \\
\hline $1 .+2.021$ & 1.250 & 3.961 & 2.450 & 3.5 & 1.405 & .4439 & 17.21 & 5.437 \\
\hline $1.5 \mid 1.951$ & 1.163 & 4.389 & 2.595 & 3.6 & 1.394 & .4305 & 18.06 & 5.580 \\
\hline$1 . 6 \longdiv { 1 . 8 9 1 }$ & 1.069 & $4.8+1$ & 2.739 & 3.7 & 1.383 & .4179 & 18.93 & 5.721 \\
\hline $1.7 \mid 1.838$ & .9965 & & 2.880 & 3.8 & 3 & .4060 & 19.82 & 5.863 \\
\hline 1.81 .791 & .9332 & 5.804 & 3.024 & 3.9 & 1.363 & .3948 & 20.73 & 6.005 \\
\hline $1.9 \sqrt[1.749]{ }$ & .8767 & 6.314 & 3.165 & 4.0 & 1.354 & .3841 & 21.67 & 6.145 \\
\hline 2.01 .711 & .8268 & 6.845 & 3.307 & 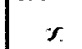 & 1 & 0 & r. & $x$ \\
\hline
\end{tabular}

Table 7. Numerical calculation of $k$ and $k$ ' for different value of $m$.

Stokes notes that for values of $\mathbf{m}>0.3$ that the values of $k$ and $k^{\prime}$ approximate an algebraic form in terms of $\mathbf{m}$ that is quite similar to that for the sphere. As such, the spherical case can likely approximate the cylinder case with appropriate choices of effective radius, a finding consistent with those of Blom et al and $\mathrm{Xu}[22,42]$. The use of the cylinder approach is also complicated, as pointed out by Lamb in declining to derive the relationships[48]:

In view of the length of the necessary investigations, and of the fact that the problems in question are inferior in interest to those which relate to a spherical boundary, we content ourselves with a reference to the original papers by Stokes. 
As shown small values of $m$ below, the values of $k$ and $k$ ' deviate quite dramatically from the spherical relationships and it becomes important to use the cylinder relationships to obtain correct force values.

This part points out one additional difference between the work by Blom et al and that by $\mathrm{Xu}$, Fletcher and the current research. The size scale for Blom et al lead to values of $\mathrm{m}$ that are in the range in which the spherical approximation is a good one. However, the small scale of the current beam operating at $50-100 \mathrm{kHz}$ leads to values of $\mathbf{m}$ that is well below 0.10 . For example, the case considered in modeling above corresponds to a value of $\mathrm{m}$ equal to 0.0533 for air at 1 atmosphere; it will decrease from this value if the pressure is reduced since kinematic viscosity decreases as pressure decreases.

All parameters used in stokes' equation, which are same as the set up in CFX modeling, are listed in Table 8. The values of k and k' are achieved using MathCad program which created by Dr. Bradshaw (see Appendix C). The drag force versus time according stokes equation is shown in Figure 55. 


\begin{tabular}{|c|c|c|}
\hline Height $(\mu \mathrm{m})$ & $\mathrm{H}$ & 2.00 \\
\hline Width $(\mu \mathrm{m})$ & W & 1.10 \\
\hline Effective diameter $(\mu \mathrm{m})$ & D & 1.67 \\
\hline Effective Radius $(\mu \mathrm{m})$ & $\mathrm{R}$ & 0.835 \\
\hline Density of air $\left(\mathrm{kg} / \mathrm{m}^{3}\right)$ & $\rho$ & 1.19 \\
\hline Viscosity $(\mathrm{kg} / \mathrm{s} \cdot \mathrm{m})$ & $\mu$ & $1.83 \mathrm{E}-05$ \\
\hline Frequency $(\mathrm{kHz})$ & $f$ & 40 \\
\hline Angular speed $(\mathrm{rad} / \mathrm{s})$ & $\omega$ & $2.51 \mathrm{E}+05$ \\
\hline Kinetic viscosity $\left(\mathrm{m}^{2} / \mathrm{s}\right)$ & $v$ & $1.55 \mathrm{E}-05$ \\
\hline Maximum displacement $(\mu \mathrm{m})$ & $\mathrm{S}_{0}$ & 0.2 \\
\hline \multirow[t]{4}{*}{$\begin{array}{l}\text { Mass of fluid replaced by } \\
\text { cylinder per unit length } \\
(\mathrm{kg} / \mu \mathrm{m})\end{array}$} & $\mathrm{m}_{\mathrm{c}}=\pi \cdot \rho \cdot \mathrm{R}^{2}$ & $2.59425 \mathrm{E}-12$ \\
\hline & $\mathbf{m}=1 / 2 \cdot R \cdot \sqrt{ }(\omega / v)$ & 0.0533 \\
\hline & $\mathrm{k}$ & 42.918 \\
\hline & $\mathrm{k}^{\prime}$ & 135.00 \\
\hline Velocity & \multicolumn{2}{|c|}{$U=-\omega S_{0} \sin (\omega t)$} \\
\hline Acceleration & \multicolumn{2}{|c|}{$U^{\prime}=-\omega^{2} S_{0} \cos (\omega t)$} \\
\hline $\mathrm{F}=-\mathrm{m}_{\mathrm{c}} \mathrm{k} U^{\prime}-\mathrm{m}_{\mathrm{c}} \mathrm{k}^{\prime} \omega \mathrm{U}$ & & \\
\hline
\end{tabular}

Table 8. Parameters used in calculation of force acting at the cylinder. 


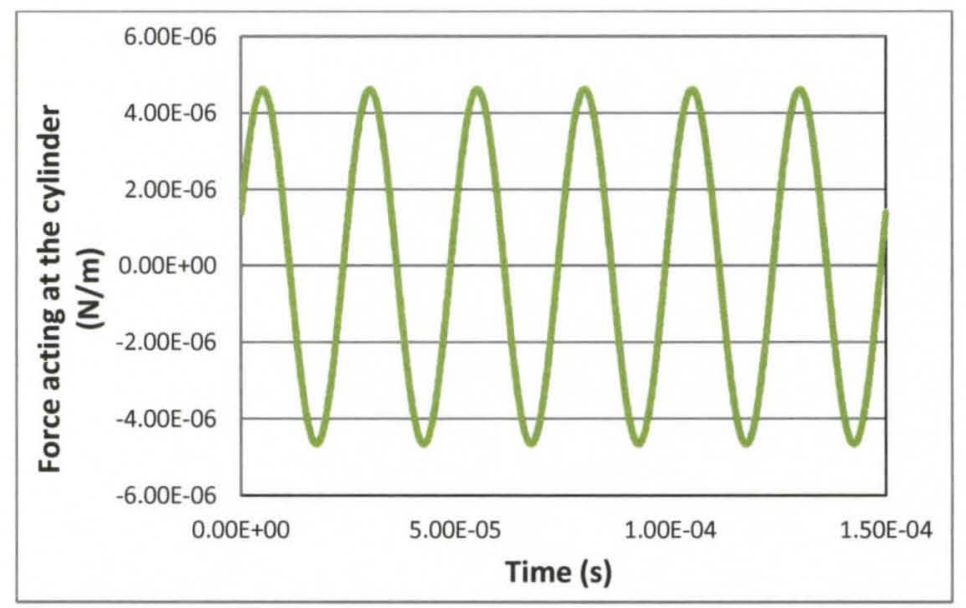

Figure 55. Force vs. Time for resonating cylinder from Stoke equation.

Drag force in $\mathrm{x}$ direction per unit length are calculated using CFX and the Stokes cylinder equation. Table 9 and Figure 56 demonstrate that these two results are in the same phase and the magnitude of maximum drag force is very close. The drag force from stoke equation is $4.63 \mu \mathrm{N} / \mathrm{m}$, and the value from the CFX calculation is $4.58 \mu \mathrm{N} / \mathrm{m}$. The variation is about $1 \%$; this indicates that the CFX model matches closely to the theory case. As such, it is reasonable to conclude that CFX can be used to determine the loads acting on a cylinder (or other cross-section shape) resonating in similar conditions.

\begin{tabular}{|c|c|c|c|c|}
\hline & \multicolumn{2}{|c|}{ Stokes model } & \multicolumn{2}{c|}{ CFX } \\
\hline & Minimum & Maximum & Minimum & Maximum \\
\hline 1 & $-4.6336 \mathrm{E}-06$ & $4.6336 \mathrm{E}-06$ & $-4.6360 \mathrm{E}-06$ & $4.2828 \mathrm{E}-06$ \\
\hline 2 & $-4.6336 \mathrm{E}-06$ & $4.6336 \mathrm{E}-06$ & $-4.5855 \mathrm{E}-06$ & $4.5322 \mathrm{E}-06$ \\
\hline 3 & $-4.6336 \mathrm{E}-06$ & $4.6336 \mathrm{E}-06$ & $-4.5681 \mathrm{E}-06$ & $4.5417 \mathrm{E}-06$ \\
\hline 4 & $-4.6336 \mathrm{E}-06$ & $4.6336 \mathrm{E}-06$ & $-4.5627 \mathrm{E}-06$ & $4.5515 \mathrm{E}-06$ \\
\hline 5 & $-4.6336 \mathrm{E}-06$ & $4.6336 \mathrm{E}-06$ & $-4.5614 \mathrm{E}-06$ & $4.5626 \mathrm{E}-06$ \\
\hline 6 & $-4.6336 \mathrm{E}-06$ & $4.6336 \mathrm{E}-06$ & $-4.5566 \mathrm{E}-06$ & $4.5567 \mathrm{E}-06$ \\
\hline
\end{tabular}

Table 9. Comparison of peak results from stoke equation and from CFX 


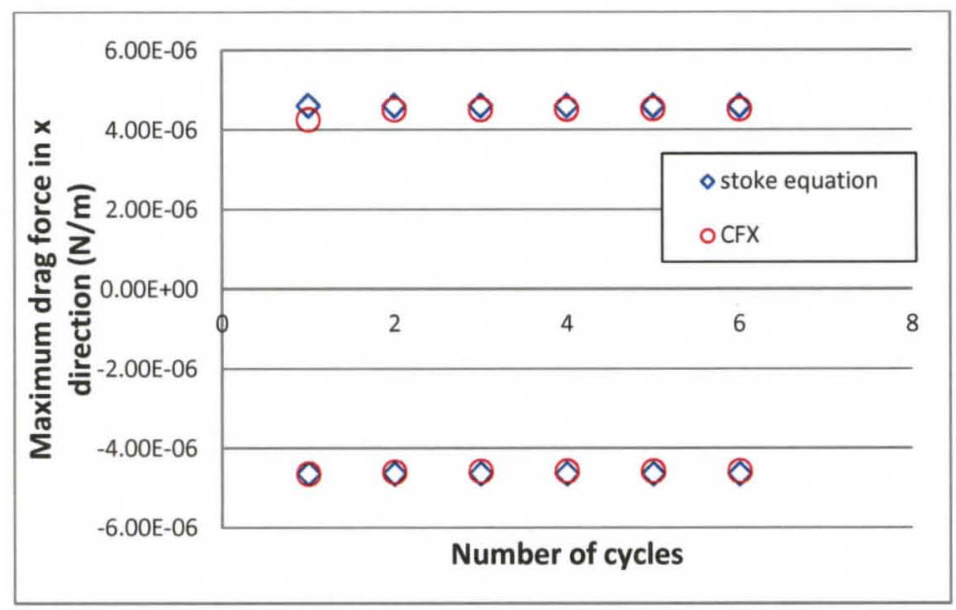

Figure 56. Comparison of results from stoke equation and from CFX.

\subsection{Curve Fit of Stokes' Oscillating Cylinder Model}

While the MathCad document referenced above and reprinted in the Appendix can perform the calculations required for $\mathrm{k}$ and k', it is tedious to embed in a standalone code (such as Matlab) to perform general vibration simulations. It is also quite involved so there are many opportunities for mistakes during coding that must be eliminated and the results carefully checked. The data generated from the MathCad document has already been fully verified to match the results provided in the original Stokes' paper so there is strong confidence in its correctness.

Instead of recreating the mathematics of the MathCad document, it was used to generate a data set that could be studied to identify a suitable curve fit that could be easily coded in any program for general vibration simulation. This approach begins with two approximations that were noted by Stokes in the original paper. For $\mathrm{m} \rightarrow 0$, the solution for k and k' can be obtained using:

$$
L(m)=\ln (m)+\gamma \quad ; \quad \gamma=0.5772157 \ldots(\text { Euler's constant })
$$




$$
k=1+\frac{\pi}{4 m^{2}} \frac{1}{L(m)^{2}+\left(\frac{\pi}{4}\right)^{2}} \quad ; \quad k^{\prime}=-\frac{1}{m^{2}} \frac{L(m)}{L(m)^{2}+\left(\frac{\pi}{4}\right)^{2}}
$$

The MathCad document was used to verify the limits at which this fit was sufficiently accurate. For the curve fitting purposes below, it was judged that this approximation would be used for $\mathrm{k}$ for $\mathrm{m} \leq 0.016$ and for $\mathrm{k}^{\prime}$ for $\mathrm{m} \leq 0.060$. The percent error between the approximation above and the full Stokes' model calculation was $1.135 \%$ for $\mathrm{k}(\mathrm{m}=0.016)$ and $-0.345 \%$ for $\mathrm{k}^{\prime}(\mathrm{m}=0.060)$; the percent error reduces from this value as $m$ decreases. Smaller limits of $m$ could be chosen that lead to less error at the associated maximum value of $m$; however, this increases the error in the curve fit that is developed below for larger values of $\mathrm{m}$. The limits chosen here were determined in a trial-and-error fashion to minimize the overall error in $\mathrm{k}$ and k' (as will be presented below).

As was mentioned in the previous section, Stokes noted that the $\mathrm{k}$ and $\mathrm{k}$ ' values developed for the cylinder model can be well approximated by functions of a form analogous to those for the oscillating sphere model when $\mathrm{m}$ was sufficiently large (on the order of $0.3-0.4$ per the original paper). The equations in this case take the form:

$$
k=1+\frac{\sqrt{2}}{m} ; \quad k^{\prime}=\frac{\sqrt{2}}{m}+\frac{1}{2 m^{2}}
$$

The MathCad document was used to verify the limits at which this fit was sufficiently accurate. The original limits from Stokes' paper corresponded to roughly $10 \%$ error, which was deemed too large for use here. Instead, the limit $m \geq 2.00$ is chosen 
for both $\mathrm{k}$ and $\mathrm{k}$ ' for the curve fit below; this corresponds to a percent error between the approximation above and the full Stokes' model calculation of $-0.236 \%$ and $0.634 \%$ for $\mathrm{k}$ and $\mathrm{k}$, respectively, at $\mathrm{m}=2.00$; the percent error decreases from these values as $\mathrm{m}$ increases.

These two approximation cases leave a region of $\mathrm{m}$ between 0.016 and 2.00 (henceforth referred to as the central region) to be modeled with a curve fit. Stokes developed the approximation for large $m$ by noting that the value $m^{2}(k-1)$ and $m^{2} k^{\prime}$ could each be approximated by a line (i.e. $A+B$, where $A$ and $B$ are suitable constants) for $m>0.30$. This inspired a similar choice for the fit in the central region; namely, the values for $k$ and $k^{\prime}$ were multiplied by $m^{p}$ where $p$ is a positive non-integer constant. The resulting data sets were plotted versus $\mathrm{m}$ for the region of interest $(0.016-$ 2.00 for $\mathrm{k} ; 0.060-2.00$ for $\left.\mathrm{k}^{\prime}\right)$. The ideal value of $\mathrm{p}$ was chosen for each data set as the one which best caused the resulting data set to appear as though it could be well-fit by a parabola. An example is shown for $\mathrm{k}^{\mathrm{p}}$ in Figure 57 below. Small values of $\mathrm{p}$ is lead to a noticeable upturn at small $\mathrm{m}$ while large values of $\mathrm{p}$ lead to a downturn at small $\mathrm{m}$ and more curvature. The smallest value of $p$ that eliminated the upturn was chosen; this was 1.55 for $\mathrm{k}$ and 2.55 for $\mathrm{k}^{\prime}$ and caused the data to appear linear or parabolic. Hence, such data could be well-fit with a polynomial.* 


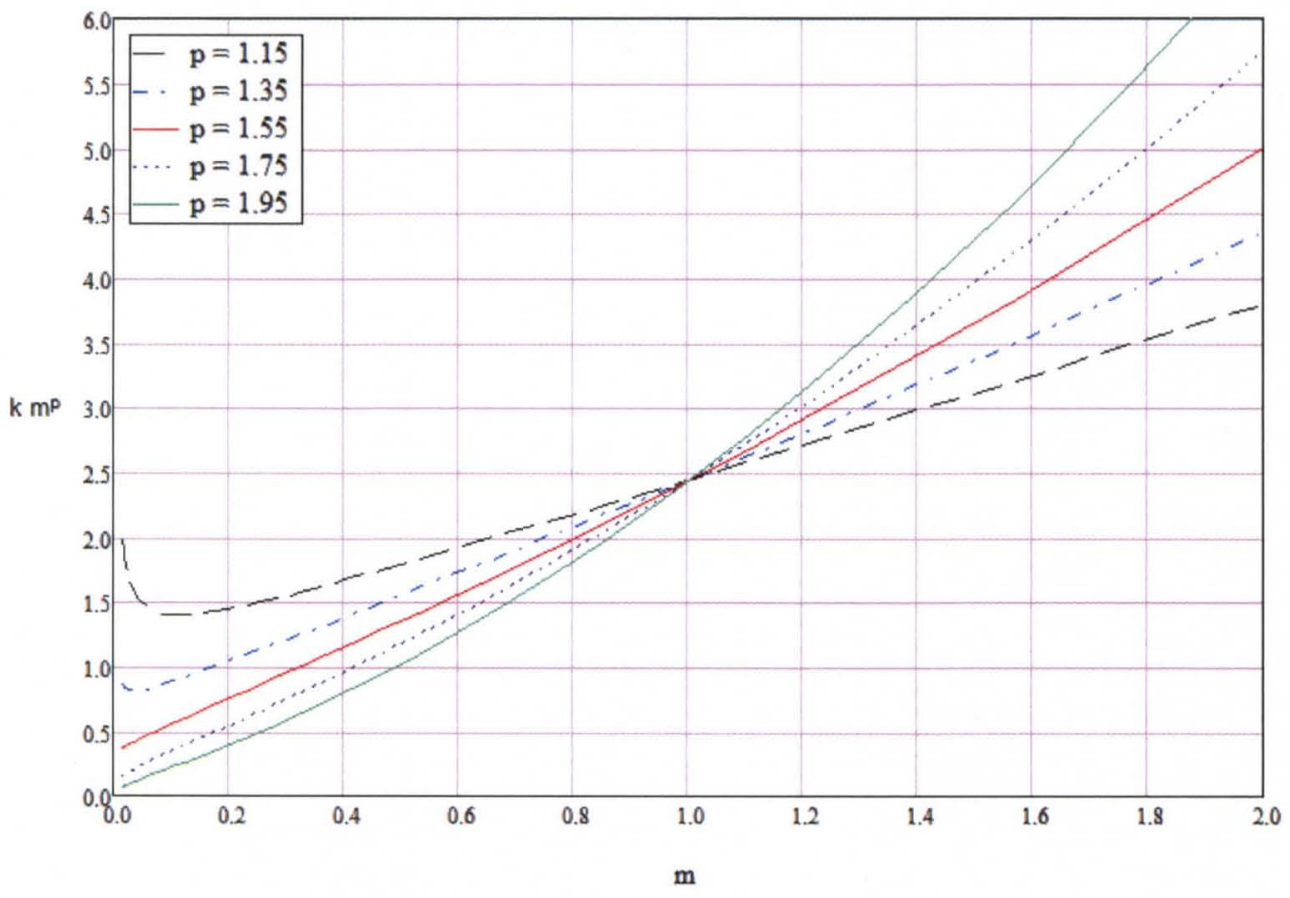

Figure 57. Values of $\mathrm{k} \mathrm{m}^{\mathrm{p}}$ for various $\mathrm{p}$ for $\mathrm{m} \in[0.016,2.00]$

Data sets were generated of $\mathrm{k} \mathrm{m}^{1.55}$ and $\mathrm{k}^{\prime} \mathrm{m}^{2.55}$ were developed for the central region between $\mathrm{m} \in\left[\mathrm{m}_{0}, 2.00\right]$ for $\mathrm{k}$ and $\mathrm{m} \in\left[\mathrm{m}^{\prime}, 2.00\right]$ for $\mathrm{k}$ ', where $\mathrm{m}_{0}$ and $\mathrm{m}_{0}$ are the central region lower limits ( 0.016 and 0.060 for $\mathrm{k}$ and k', respectively). These data sets were then fit with a polynomial of the form:

$$
\begin{aligned}
& f(m)=c_{0}+c_{1}\left(m-m_{0}\right)+c_{2}\left(m-m_{0}\right)^{2}+c_{3}\left(m-m_{0}\right)^{3} \\
& f^{\prime}(m)=c_{0}^{\prime}+c_{1}^{\prime}\left(m-m_{0}^{\prime}\right)+c_{2}^{\prime}\left(m-m_{0}^{\prime}\right)^{2}+c_{3}^{\prime}\left(m-m_{0}^{\prime}\right)^{3}
\end{aligned}
$$

where $f(m)$ and $f^{\prime}(m)$ are the optimal fitting functions for the data sets developed for $\mathrm{k}$ and $\mathrm{k}^{\prime}$, respectively; the coefficients $c_{1}, c_{2}, c_{3}, c^{\prime}{ }_{1}, c^{\prime}{ }_{2}$ and $c^{\prime}{ }_{3}$ were developed using the generalized fitting function in MathCad with the values of $c_{0}$ and $c^{\prime}{ }_{0}$ set such that the approximation in the central region will be continuous with that for small values of $\mathrm{m}$ at 
the corresponding lower limit $\left(\mathrm{m}_{0}\right.$ or $\left.\mathrm{m}^{\prime}{ }_{0}\right)$. The values are given in Table 10 below. The parameters $\mathrm{k}(\mathrm{m})$ and $\mathrm{k}^{\prime}(\mathrm{m})$ can then be approximated as:

$$
k(m)=\left\{\begin{array}{cc}
1+\frac{\pi}{4 m^{2}} \frac{1}{L(m)^{2}+\left(\frac{\pi}{4}\right)^{2}} & m \leq m_{0} \\
m^{-p}\left[\sum_{j=0}^{3} c_{j}\left(m-m_{0}\right)^{j}\right] & m_{0}<m<2.00 \\
1+\frac{\sqrt{2}}{m} & m \geq 2.00
\end{array} k^{\prime}(m)=\left\{\begin{array}{cc}
\frac{1}{m^{2}} \frac{L(m)}{L(m)^{2}+\left(\frac{\pi}{4}\right)^{2}} & m \leq m_{0} \\
m^{-p^{\prime}}\left[\sum_{j=0}^{3} c_{j}^{\prime}\left(m-m_{0}^{\prime}\right)^{j}\right. & m_{0}<m<2.00 \\
\frac{\sqrt{2}}{m}+\frac{1}{2 m^{2}} & m \geq 2.00
\end{array}\right.\right.
$$

where $\mathrm{L}(\mathrm{m})$ is the function given previously. 


\begin{tabular}{|c|c|c|c|}
\hline \multicolumn{2}{|c|}{ Fit For k(m) } & \multicolumn{2}{c|}{ Fit For k'(m) } \\
\hline Label & Value & Label & Value \\
\hline$m_{0}$ & 0.016 & $m^{\prime}{ }_{0}$ & 0.060 \\
\hline$c_{0}$ & 0.38199 & $c^{\prime}{ }{ }^{\prime}$ & 0.08471 \\
\hline$c_{1}$ & 1.97554 & $c^{\prime}{ }_{1}$ & 1.26919 \\
\hline$c_{2}$ & 0.06255 & $c^{\prime}{ }_{2}$ & 0.73687 \\
\hline$c_{3}$ & 0.06052 & $c^{\prime}{ }_{3}$ & -0.06579 \\
\hline$p$ & 1.55 & $p^{\prime}$ & 2.55 \\
\hline
\end{tabular}

Table 10. Fitting model coefficients for Stokes' cylinder model

The functions for $\mathrm{k}$ and $\mathrm{k}$ ' as functions are $\mathrm{m}$ are plotted below in Figure 58 and Figure 61 , respectively, using the approximate functions above; the results are virtually indistinguishable from the original Stokes' model equation values (not shown). One way to compare the approximation above to the original Stokes' model equations is in terms of the percentage error between the approximation and the original equations; this is shown in Figure 59 and Figure 61, respectively, for k and k'. Also shown in Figure 58 and Figure 61 are the spherical model approximations (i.e. using the function above corresponding to $m \geq 2.00$ for all values of $m$ ); it is clear that significant error occurs when the sphere approximation is used for values of $\mathrm{m}<0.3-1.0$. 


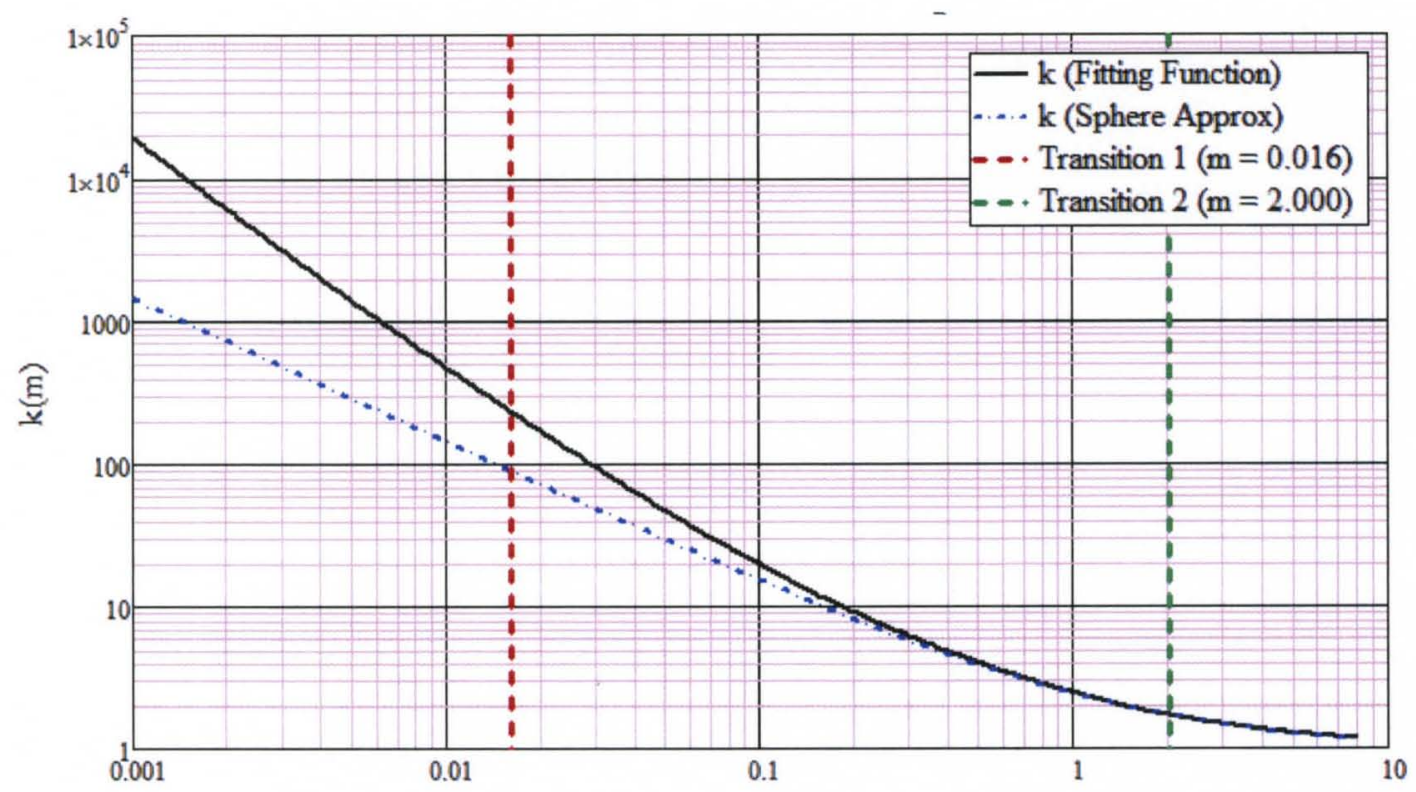

Figure 58. Value of $\mathrm{k}$ using fitting function and using spherical approximation

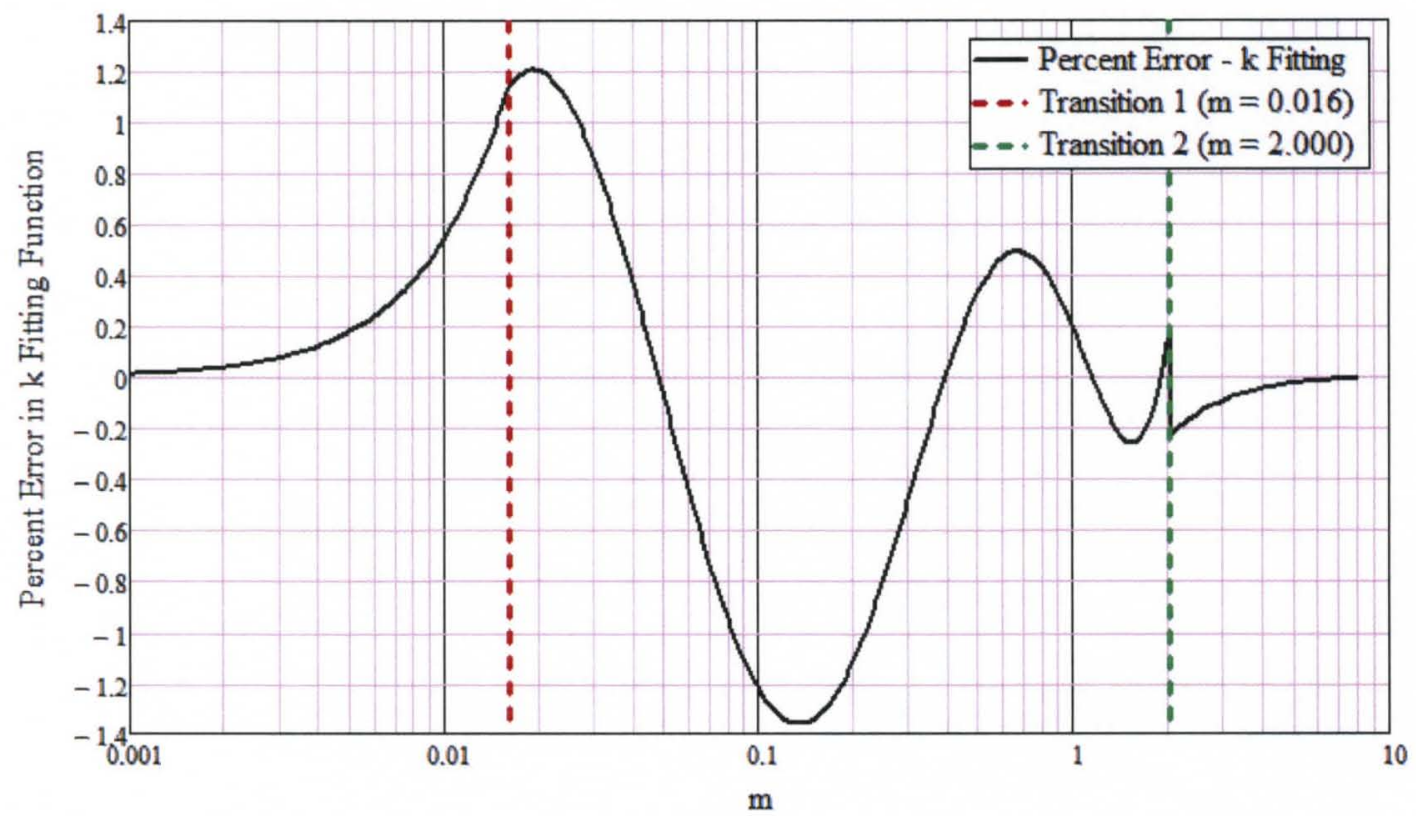

Figure 59. Percent error between fitting function for $\mathrm{k}$ and $\mathrm{k}$ from Stokes' model 


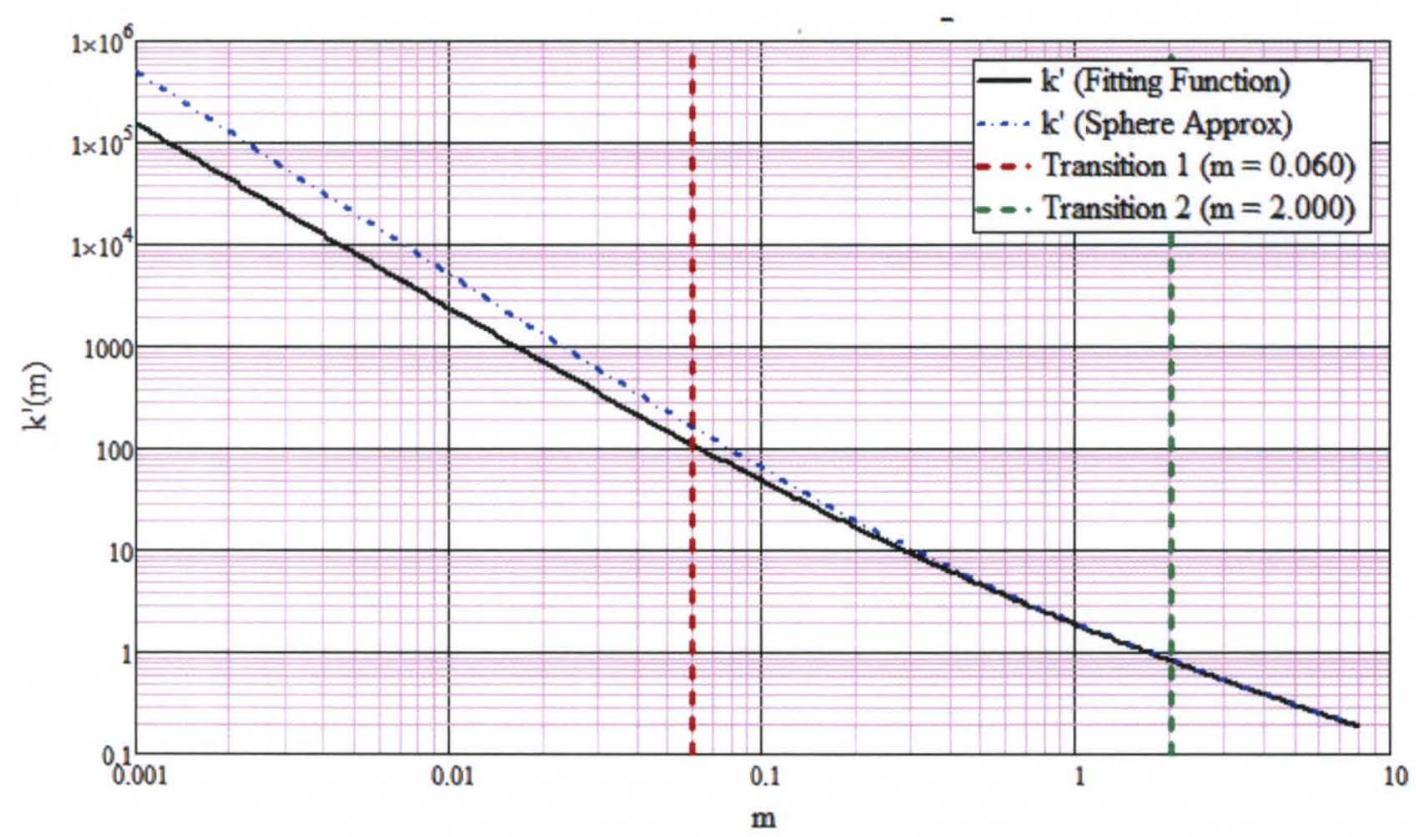

Figure 60. Value of k' using fitting function and using spherical approximation

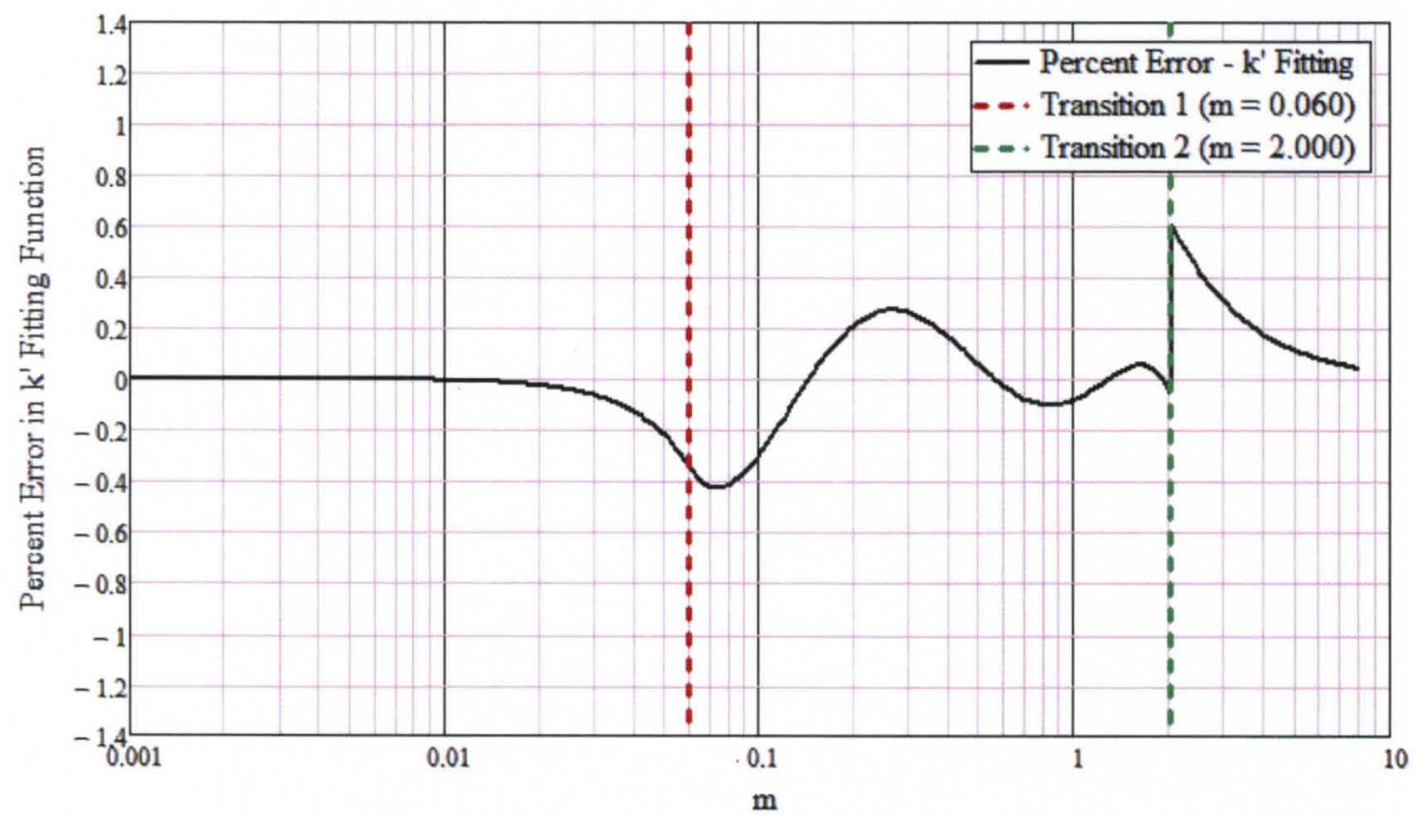

Figure 61. Percent error between fitting function for k' and k' from Stokes' model

The approximations above can be used to predict $\mathrm{k}(\mathrm{m})$ and $\mathrm{k}^{\prime}(\mathrm{m})$ for general vibration simulations. These studies also indicate the difficulties that occur with the 
spherical model approximation when applied to cylinders if $m$ is sufficiently small. Many of the device configurations considered in this dissertation will violate this condition, as $\mathrm{m}$ is well less than 0.1 for most cases.

Note that the analysis and text in this section was largely done by the Dr. Roger Bradshaw, the Ph.D. advisor of the author.

\subsection{Microcantilever Beam Fluid Simulation}

The results in the Section 3.3 represent a first step in modeling the beam loads that occur during oscillatory motion via CFD; the resulting loads are verified by Stokes theory. The next step focuses on beams with size and properties similar to those of the microcantilever sensor. The goal of these studies is to consider four fundamental cases:

1) Cylinder oscillating without a floor

2) Cylinder oscillating with a floor

3) Rectangle oscillating without a floor

4) Rectangle oscillating with a floor

In each of these cases, the analysis is performed with several velocities and frequencies. This permits the assessment of a model of beam forces expressed in terms of the beam velocity and acceleration (similar to existing analytical models). Comparisons between analytical models will also be pursued; these are the Stokes' solutions for an oscillating sphere and an oscillating cylinder produced in the last section. The results will then be used to assess whether the analytical models can be used to approximate the observed behaviors with suitable changes. For example, perhaps the Stokes cylinder model using an effective diameter will represent the data for a rectangular cross-section operating without a floor. 


\subsubsection{Cylinder Oscillating Without a Floor}

All models discussed in Sections 3.3 considered cylinder oscillating without a floor. The results verified that beam forces could be expressed in terms of the beam velocity and acceleration. To improve accuracy, the $150 \mathrm{D}$ dimension will be used in this section; this has force about $4.61 \mu \mathrm{N} / \mathrm{m}$, which is closer to the theory value than $100 \mathrm{D}$ dimension set-up. Although this increases solution time somewhat, the gain in accuracy will benefit in the expression of drag force for different cases. Figure 63 shows drag force acting on the cylinder in $\mathrm{x}$ direction changes sinusoidally with time. . Figure 62 shows the mesh for case of cylinder resonating in middle. In following sections, the effect of the change the shape of the beam cross-section and the effect of wall will be considered by comparing the drag force between different cases

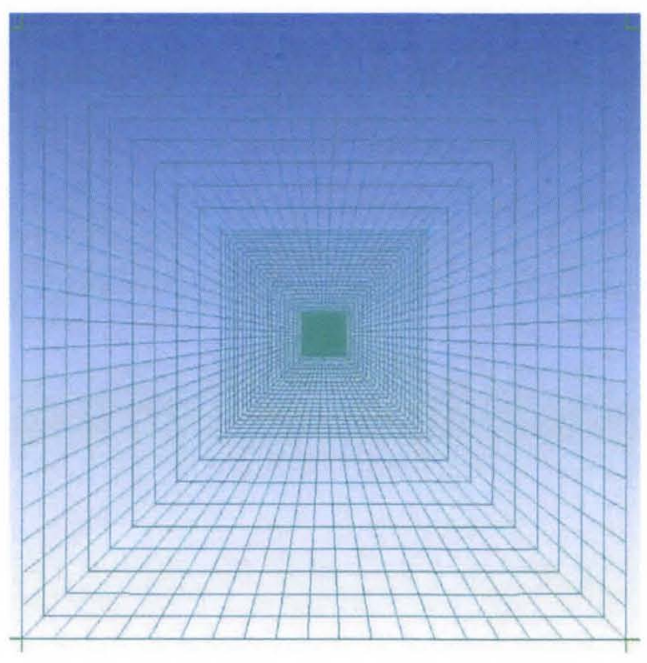

Figure 62. Mesh for case of cylinder resonating in middle 


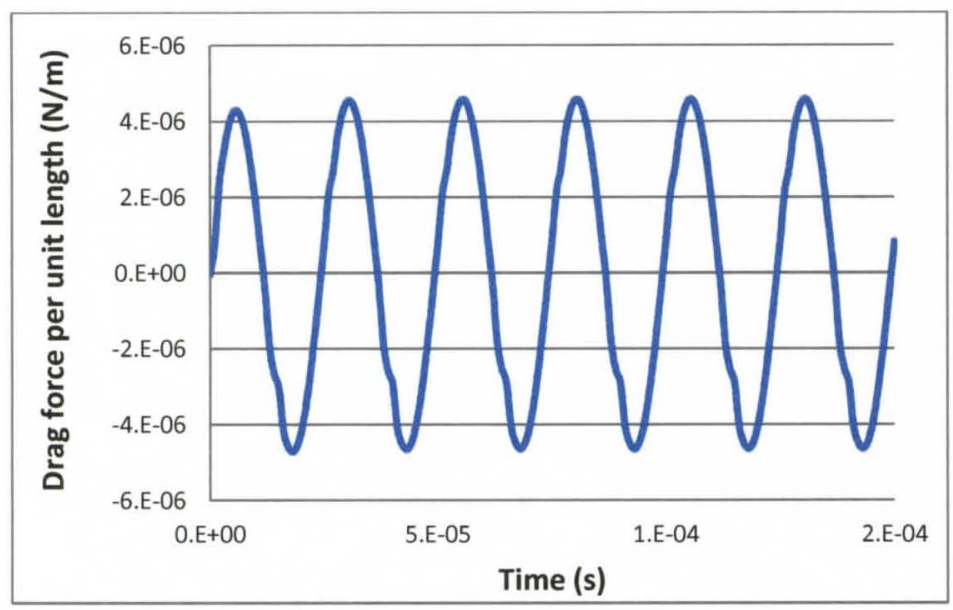

Figure 63. Drag force per unit length vs. time for case of cylinder resonating in middle

\subsubsection{Cylinder Oscillating With a Floor}

In this case the cylinder is moved to the bottom of the tank, $2 \mu \mathrm{m}$ above the bottom. Alternately the distance between cylinder and bottom could be reduced to $2 \mu \mathrm{m}$ to increase the computational economy; this was not considered to avoid needing to repeat the effect of overall model size on CFD results done in Section 3.3. The mesh density and element size is kept the same as previous case. The mesh file for case of cylinder resonating with floor is shown in Figure 64. 


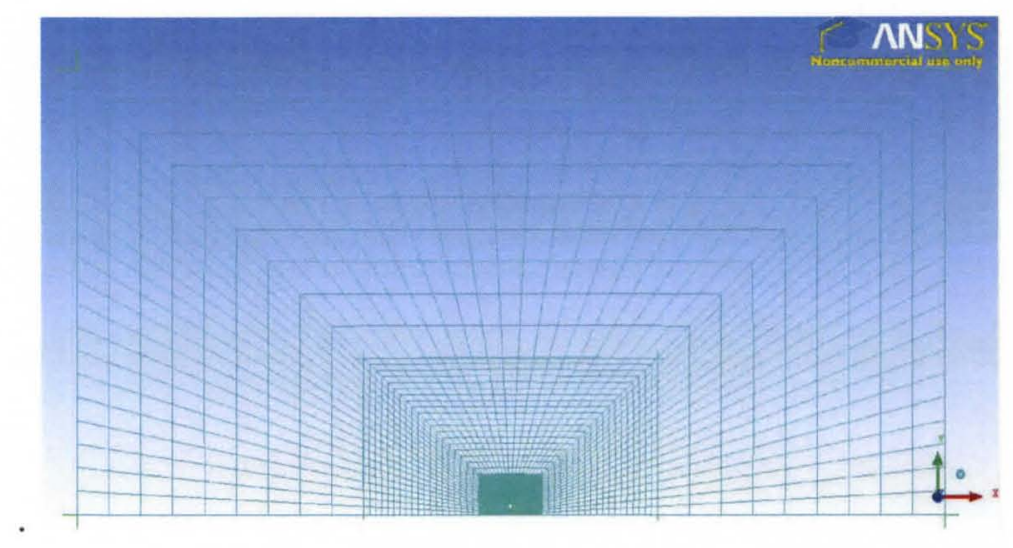

Figure 64. Mesh for cylinder resonating with floor

Drag force per unit length is calculated through CFX and plotted in Figure 65. The curve of drag force verse time for cylinder with floor is not entirely sinusoidal, perhaps due to the timestep of $10 \mathrm{e}-7$ not being sufficient for this case. The maximum drag force in each cycle compared between case of cylinder with floor and case of cylinder without floor. In Table 11 and Figure 66, the value of drag force for case of cylinder with floor is $6.45 \mu \mathrm{N} / \mathrm{m}$ which is significantly larger than the value of drag force for case of cylinder without floor $(4.58 \mu \mathrm{N} / \mathrm{m})$. The effect of viscous effects due to the floor makes the cylinder harder to translate due to force acting on it. 


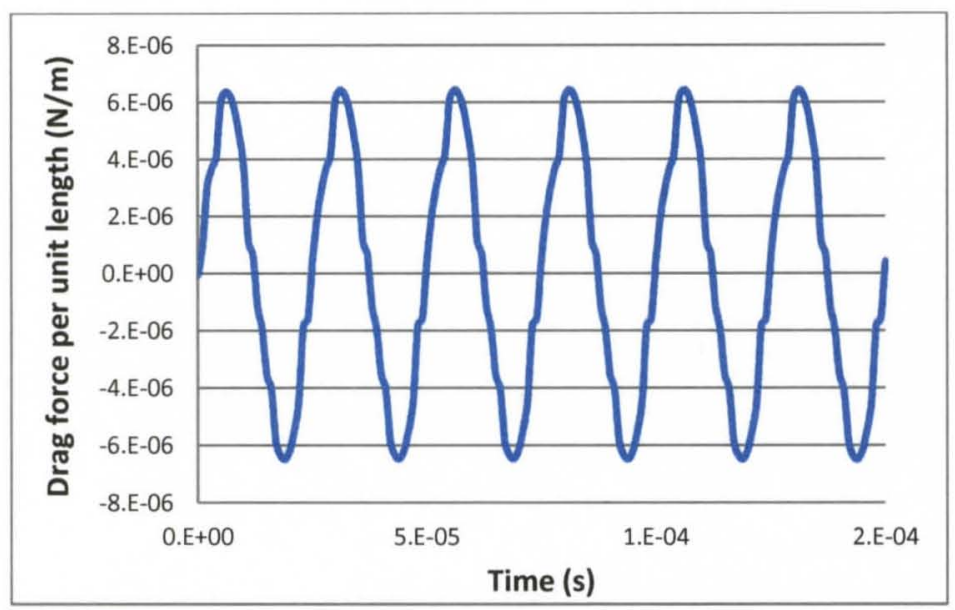

Figure 65. Drag force per unit length vs. time for case of cylinder resonating with floor

\begin{tabular}{|r|r|r|l|r|}
\hline & \multicolumn{3}{|l|}{ Cylinder with floor } & \multicolumn{2}{l|}{ Cylinder without floor } \\
\hline & \multicolumn{1}{|l|}{ Minimum } & Maximum & Minimum & \multicolumn{1}{l|}{ Maximum } \\
\hline 1 & $-4.57894 \mathrm{E}-06$ & $4.2327 \mathrm{E}-06$ & $-4.57894 \mathrm{E}-06$ & $4.2327 \mathrm{E}-06$ \\
\hline 2 & $-4.52599 \mathrm{E}-06$ & $4.46797 \mathrm{E}-06$ & $-4.52599 \mathrm{E}-06$ & $4.46797 \mathrm{E}-06$ \\
\hline 3 & $-4.51018 \mathrm{E}-06$ & $4.49784 \mathrm{E}-06$ & $-4.51018 \mathrm{E}-06$ & $4.49784 \mathrm{E}-06$ \\
\hline 4 & $-4.50923 \mathrm{E}-06$ & $4.50439 \mathrm{E}-06$ & $-4.50923 \mathrm{E}-06$ & $4.50439 \mathrm{E}-06$ \\
\hline 5 & $-4.49903 \mathrm{E}-06$ & $4.50349 \mathrm{E}-06$ & $-4.49903 \mathrm{E}-06$ & $4.50349 \mathrm{E}-06$ \\
\hline 6 & $-4.50753 \mathrm{E}-06$ & $4.50453 \mathrm{E}-06$ & $-4.50753 \mathrm{E}-06$ & $4.50453 \mathrm{E}-06$ \\
\hline
\end{tabular}

Table 11. Peak of drag force compared between cylinder without floor and with floor

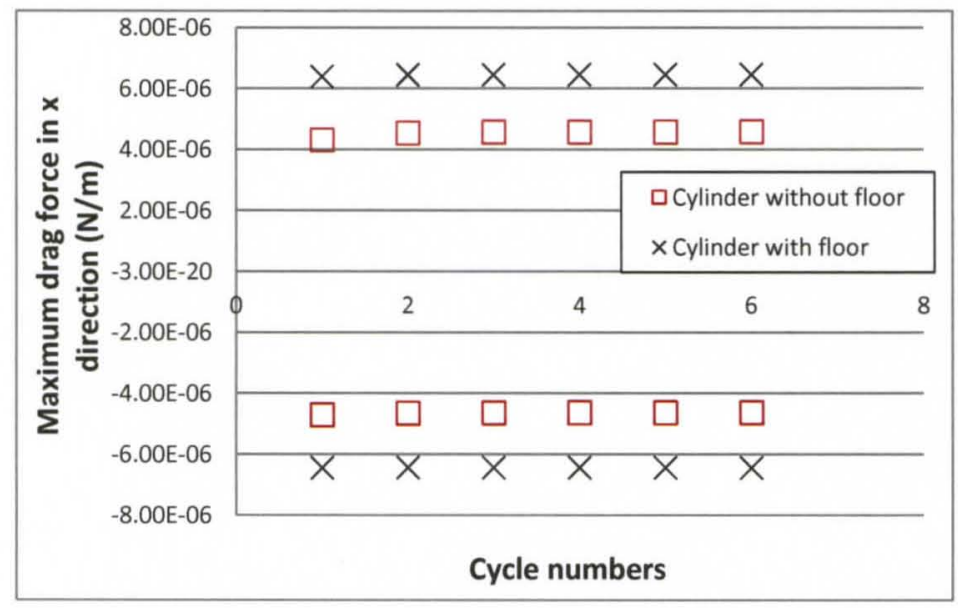

Figure 66. Peak of drag force compared between cylinder without floor and with floor 


\section{$\underline{\text { 3.6.3 Rectangle Oscillating Without a Floor }}$}

In this section, the relationship between shape of cross section and the calculation result is presented. The shape of cross section changes from cylinder to rectangle (width $1.1 \mu \mathrm{m} x$ length $2 \mu \mathrm{m}$ ) which is shown in Figure 67. The cross-section areas of the cylinder and rectangle are indentical. The drag force acting on the rectangle also changes sinusoidally versus time as shown in Figure 68; the maximum value of drag force for each cycle is $5.06 \mu \mathrm{N} / \mathrm{m}$ which a little bigger than cylinder case without a floor.

The comparison of maximum value of drag force for each cycle is listed in Table 12 and Figure 69 to show the relationship between the shape of cross section and results.

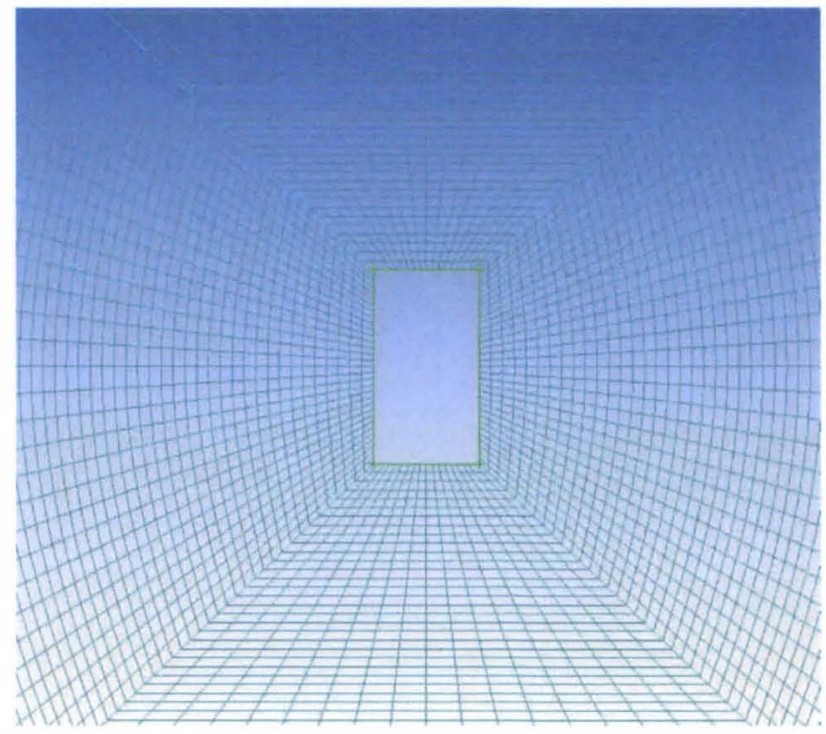

Figure 67. Mesh for case of rectangle without floor 


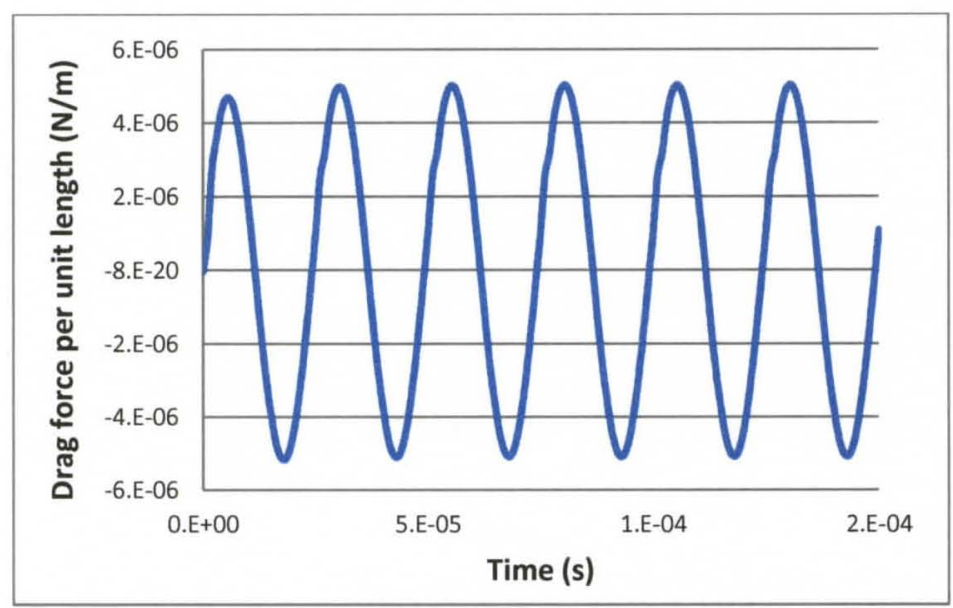

Figure 68. Drag force per unit length vs. time for case of rectangle resonating without floor

\begin{tabular}{|r|r|r|l|r|}
\hline & \multicolumn{2}{|l|}{ Rectangluar without floor } & \multicolumn{2}{l|}{ Cylinder without floor } \\
\hline & \multicolumn{1}{|l|}{ Minimum } & Maximum & Minimum & Maximum \\
\hline 1 & $-5.1096 \mathrm{E}-06$ & $4.70422 \mathrm{E}-06$ & $-4.57894 \mathrm{E}-06$ & $4.2327 \mathrm{E}-06$ \\
\hline 2 & $-5.0426 \mathrm{E}-06$ & $4.98702 \mathrm{E}-06$ & $-4.52599 \mathrm{E}-06$ & $4.46797 \mathrm{E}-06$ \\
\hline 3 & $-5.0372 \mathrm{E}-06$ & $5.01375 \mathrm{E}-06$ & $-4.51018 \mathrm{E}-06$ & $4.49784 \mathrm{E}-06$ \\
\hline 4 & $-5.03255 \mathrm{E}-06$ & $5.02735 \mathrm{E}-06$ & $-4.50923 \mathrm{E}-06$ & $4.50439 \mathrm{E}-06$ \\
\hline 5 & $-5.0317 \mathrm{E}-06$ & $5.02775 \mathrm{E}-06$ & $-4.49903 \mathrm{E}-06$ & $4.50349 \mathrm{E}-06$ \\
\hline 6 & $-5.0308 \mathrm{E}-06$ & $5.03665 \mathrm{E}-06$ & $-4.50753 \mathrm{E}-06$ & $4.50453 \mathrm{E}-06$ \\
\hline
\end{tabular}

Table 12. Peak of drag force compared between rectangle without floor and cylinder without floor 


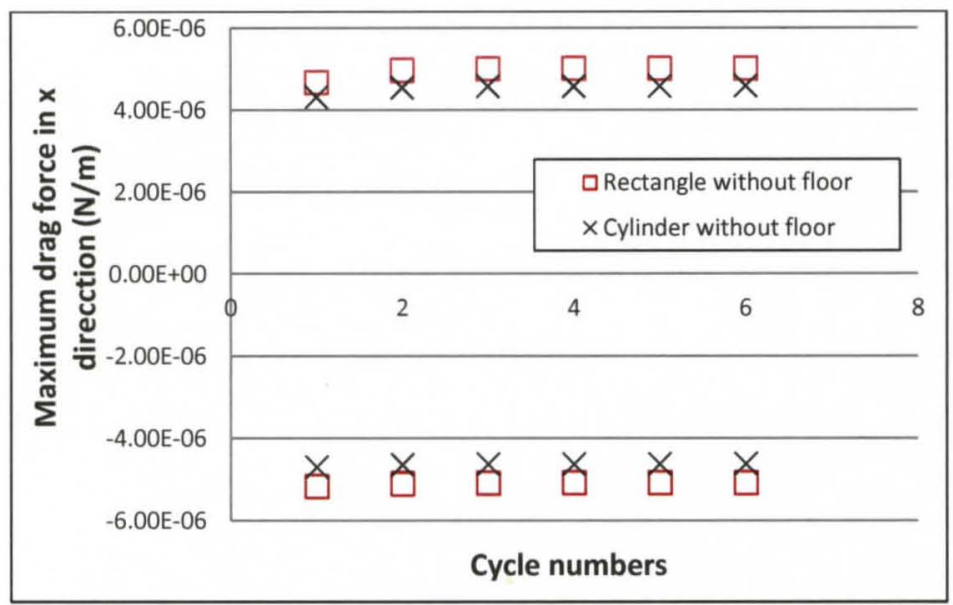

Figure 69. Peak of drag force compared between rectangle without floor and cylinder without floor

\subsubsection{Rectangle Oscillating With a Floor}

In this case, the shape of beam cross section is rectangular which is resonating 2 $\mu \mathrm{m}$ above a floor. This case simulates the real working environment for the pizeoresistive microcantilever beam. This case could be obtained ideally change case via a cylinder without floor to cylinder with floor (adding effect of wall effects in this step) then to a rectangular with floor (adding change of cross section). The mesh file for case of rectangle resonating with floor is shown in Figure 70. As in the cylinder with floor case, the half of the tank is used to speed the calculation. 


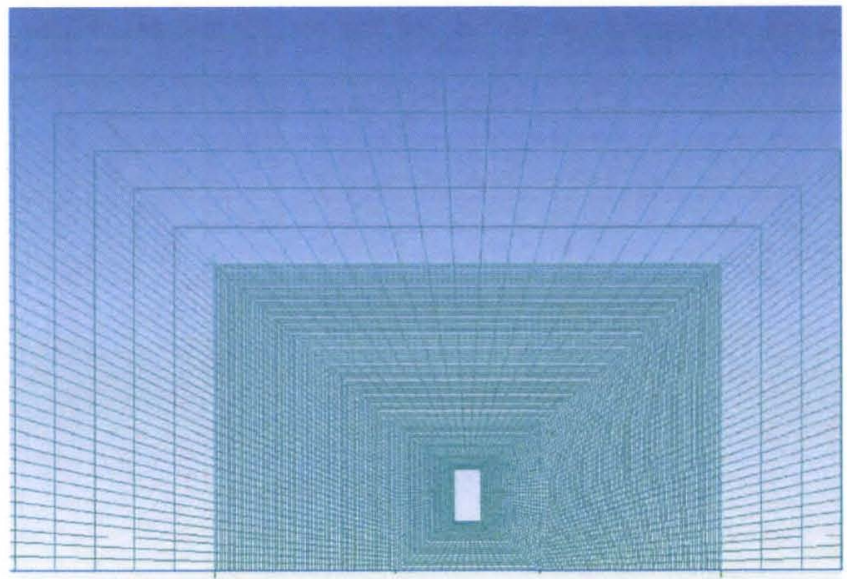

Figure 70 Mesh for case of rectangle resonating with floor

The drag force per unit length is plotted in Figure 71. The drag force versus time is not perfectly sinusoidal, which may be due to the non-sufficient time step set-up. As with the cylinder, the rectangle with floor has a bigger drag force value then the rectangle without floor due to the effect of wall viscosity. The comparison of drag force per unit length for each cycle is shown in Table 13 and Figure 72.

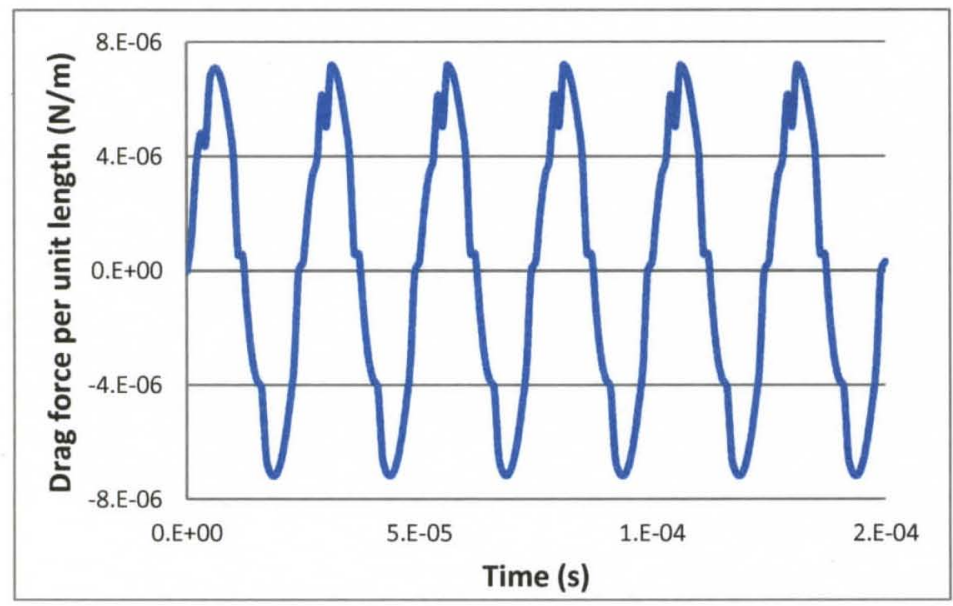

Figure 71. Drag force per unit length vs. time for case of rectangle with floor 


\begin{tabular}{|c|c|c|c|c|}
\hline & \multicolumn{2}{|c|}{ Rectangle without floor } & \multicolumn{2}{c|}{ Rectangle with floor } \\
\hline & Minimum & Maximum & Minimum & Maximum \\
\hline 1 & $-5.1096 \mathrm{E}-06$ & $4.70422 \mathrm{E}-06$ & $-7.4099 \mathrm{E}-06$ & $7.29785 \mathrm{E}-06$ \\
\hline 2 & $-5.0426 \mathrm{E}-06$ & $4.98702 \mathrm{E}-06$ & $-7.3241 \mathrm{E}-06$ & $7.3218 \mathrm{E}-06$ \\
\hline 3 & $-5.0372 \mathrm{E}-06$ & $5.01375 \mathrm{E}-06$ & $-7.32275 \mathrm{E}-06$ & $7.38195 \mathrm{E}-06$ \\
\hline 4 & $-5.03255 \mathrm{E}-06$ & $5.02735 \mathrm{E}-06$ & $-7.3595 \mathrm{E}-06$ & $7.35115 \mathrm{E}-06$ \\
\hline 5 & $-5.0317 \mathrm{E}-06$ & $5.02775 \mathrm{E}-06$ & $-7.3871 \mathrm{E}-06$ & $7.35255 \mathrm{E}-06$ \\
\hline 6 & $-5.0308 \mathrm{E}-06$ & $5.03665 \mathrm{E}-06$ & $-7.3658 \mathrm{E}-06$ & $7.3083 \mathrm{E}-06$ \\
\hline
\end{tabular}

Table 13. Drag force compared between case rectangle with or without floor

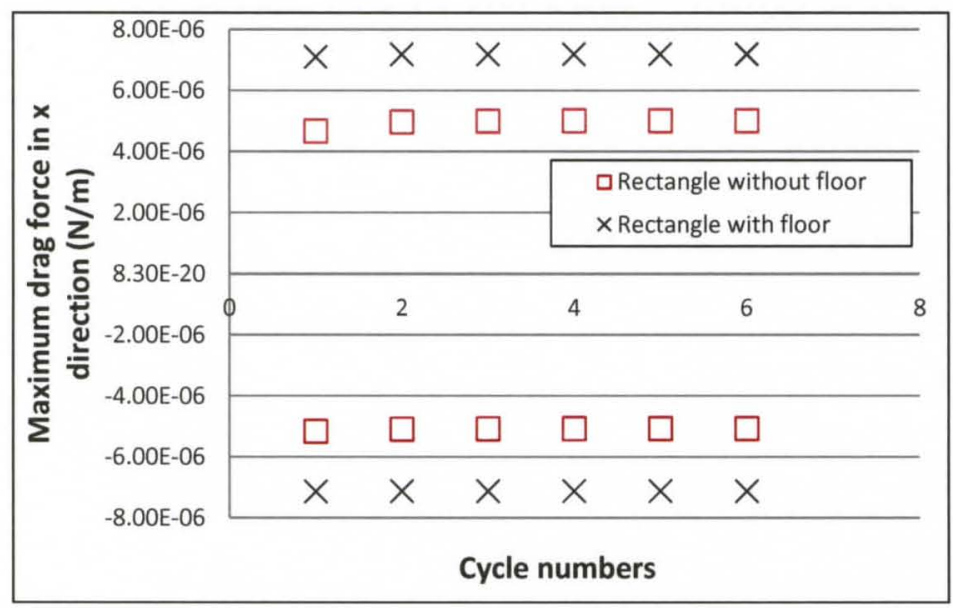

Figure 72. Drag force compared between case rectangle with or without floor

\section{$\underline{3.6 .5 \text { Comparison }}$}

Drag forces for four cases discussed above are listed in Table 14. The middle column considers of cylinder ratio of (cylinder with floor to cylinder without floor), and cases of rectangle ratio of (rectangle with floor and rectangle without floor). This considering common shape of cross section with only the effect of wall viscosity. A single factor could be achieved by considering common floor cases, as ratios cylinder/rectangle with floor and cylinder/rectangle without floor shown in the right column of Table 14. For cylinder group, the factor is 1.4083 ; for rectangle group, the 
factor is 1.4274 . The factor of the cylinder group and the rectangle group are very close. This means that the drag force increased significantly with floor presented. The minor difference between them may caused by difference shape of cross section or the chose time step.

\begin{tabular}{|c|c|c|c|}
\hline & $\begin{array}{c}\text { Peak drag force per } \\
\text { unit length }(\mu \mathrm{N} / \mathrm{m})\end{array}$ & $\begin{array}{c}\text { Effect of floor (with } \\
\text { floor/without floor) }\end{array}$ & $\begin{array}{c}\text { Effect of shape } \\
\text { (Rectangle/cylinder) }\end{array}$ \\
\hline Cylinder without floor & 4.58 & 1.4083 & 1.0983 \\
(A) & (C/A) & 1.1132 \\
\hline Cylinder with floor & 6.45 & & (D/B) \\
\hline Rectangle without floor & 5.03 & 1.4274 & \\
(C) & 7.18 & $(\mathrm{D} / \mathrm{C})$ & \\
\hline (D) & & & \\
\hline
\end{tabular}

Table 14.Summary of drag force for four cases

By grouping the without floor cases (cylinder without floor and rectangle without floor) and the with floor cases (cylinder with floor and cylinder without floor), the effect of wall viscosity, and effect from shape of cross-section is left over. Another factor could also be achieved by dividing the rectangle with/without floor by the cylinder with/without floor. For the without floor group, the factor due to cross-section shape is 1.0983 , while the with floor group is 1.1132 . Both of these are very close to 1 , which means the change of shape of cross section has a real but minor effect on the result. Note that these realist 
are set up the resonating frequency of $40 \mathrm{kHz}$. The next section, investigates the relationship between drag force and different velocities (via resonating frequencies).

\subsubsection{Effect of Different Frequency (Velocity)}

The four cases discussed above are performed with several frequencies, such as $30 \mathrm{kHz}, 40 \mathrm{kHz}, 60 \mathrm{kHz}, 80 \mathrm{kHz}$, and $90 \mathrm{kHz}$. The purpose of doing so is achieving a force expression in terms of the beam velocity and acceleration. Considering the set up of time-steps in CFX is an important factor which will cause variation in results; therefore the time-steps for different frequencies is chosen according to the number of the time steps. Time steps of 25 is chosen for each frequency, and the time during $(T=1 / f)$ will divide by the number of time steps. The answer of this division will be determined as set up of time-steps for each frequency. The set up of time-steps for each frequency is showed in Table 15.

\begin{tabular}{|c|l|l|l|c|}
\hline Frequency $(\mathrm{Hz})$ & Time Duration $(\mathrm{s})$ & Steps & Time steps & Time of 6 cycles(s) \\
\hline 30000 & $3.33 \mathrm{E}-05$ & 25 & $1.33 \mathrm{E}-06$ & $2.00 \mathrm{E}-04$ \\
\hline 40000 & $2.50 \mathrm{E}-05$ & 25 & $1.00 \mathrm{E}-06$ & $1.50 \mathrm{E}-04$ \\
\hline 50000 & $2.00 \mathrm{E}-05$ & 25 & $8.00 \mathrm{E}-07$ & $1.20 \mathrm{E}-04$ \\
\hline 60000 & $1.67 \mathrm{E}-05$ & 25 & $6.67 \mathrm{E}-07$ & $1.00 \mathrm{E}-04$ \\
\hline 70000 & $1.43 \mathrm{E}-05$ & 25 & $5.71 \mathrm{E}-07$ & $8.57 \mathrm{E}-05$ \\
\hline 80000 & $1.25 \mathrm{E}-05$ & 25 & $5.00 \mathrm{E}-07$ & $7.50 \mathrm{E}-05$ \\
\hline 90000 & $1.11 \mathrm{E}-05$ & 25 & $4.44 \mathrm{E}-07$ & $6.67 \mathrm{E}-05$ \\
\hline
\end{tabular}

Table 15. Setup of time-steps for different frequencies 
The model chosen in calculation has following set-ups: 150D (box dimension is 150 times bigger than cylinder's diameter), 20 elements (elements number used to divide quarter of circle, or one edge of rectangle), level 1 (mesh refine level), time-steps of le-6s (calculation steps in CFX keeps 25 for each case), and $\mathrm{S}_{0}$ of $0.2 \mu \mathrm{m}$ ( $\mathrm{S}_{0}$ is the maximum displacement of beam). Four cases (cylinder without floor, rectangle without floor, cylinder with floor, and rectangle with floor) were all tested in five different frequencies $(30 \mathrm{kHz}, 40 \mathrm{kHz}, 60 \mathrm{kHz}, 80 \mathrm{kHz}$, and $90 \mathrm{kHz})$. The peak force acting on the beam in the $\mathrm{x}$ direction is used as the key test result to compare. The force acting on the beam in the $\mathrm{x}$ direction is achieved from CFX-Post; this is then divided by the thickness of the model (z) to obtain force per unit length. The thickness of the model is selected automatically by CFX during extrusion; its value does not affect the force results when $\mathrm{S}_{0}$ normalized. The value of $z$ is $3 e-6 \mu \mathrm{m}$ for cylinder without floor and rectangle without floor; and 1.53e-6 $\mu \mathrm{m}$ for cylinder with floor and rectangle with floor. The value changed due to the difference size of the box. Which changed from square of $150 \mathrm{D} \times 150 \mathrm{D}$ to rectangle of $150 \mathrm{D} \times(150+\Delta)$. The change is reasonable because the $(1 / 2)^{*} 150 \mathrm{D}$ from top in the cans without floor is big enough to ignore any viscous effect from the top wall. Therefore the floor model keeps this distance on top and only changes the distance between the beam and the bottom well. The force acting on the beam is listed in Table 16 for different cases and different frequencies. The value of peak force listed in the table is the maximum force of a resonating cycle and has units of Newton per meter. 


\begin{tabular}{|c|c|c|c|c|}
\hline $\begin{array}{l}\text { Frequency } \\
(\mathrm{kHz})\end{array}$ & $\begin{array}{l}\text { Cylinder without } \\
\text { floor }\end{array}$ & $\begin{array}{l}\text { Rectangle } \\
\text { without floor }\end{array}$ & $\begin{array}{l}\text { Cylinder with } \\
\text { floor }\end{array}$ & $\begin{array}{l}\text { Rectangle with } \\
\text { floor }\end{array}$ \\
\hline 30 & $3.28 \mathrm{E}-06$ & $3.57 \mathrm{E}-06$ & $4.64 \mathrm{E}-06$ & $5.20 \mathrm{E}-06$ \\
\hline 40 & $4.61 \mathrm{E}-06$ & $5.05 \mathrm{E}-06$ & $6.45 \mathrm{E}-06$ & $7.16 \mathrm{E}-06$ \\
\hline 60 & $7.51 \mathrm{E}-06$ & $8.28 \mathrm{E}-06$ & $9.98 \mathrm{E}-06$ & $1.12 \mathrm{E}-05$ \\
\hline 80 & $1.06 \mathrm{E}-05$ & $1.18 \mathrm{E}-05$ & $1.37 \mathrm{E}-05$ & $1.54 \mathrm{E}-05$ \\
\hline 90 & $1.22 \mathrm{E}-05$ & $1.37 \mathrm{E}-05$ & $1.56 \mathrm{E}-05$ & $1.76 \mathrm{E}-05$ \\
\hline
\end{tabular}

Table 16. Peak drag force per unit length $(\mathrm{N} / \mathrm{m})$ for four cases and different frequencies The drag forces per unit length of each case for different frequencies also are plotted in Figure 73. It is clear that the drag force varies linearly with frequencies regardless of the cross section or the presence of the wall.

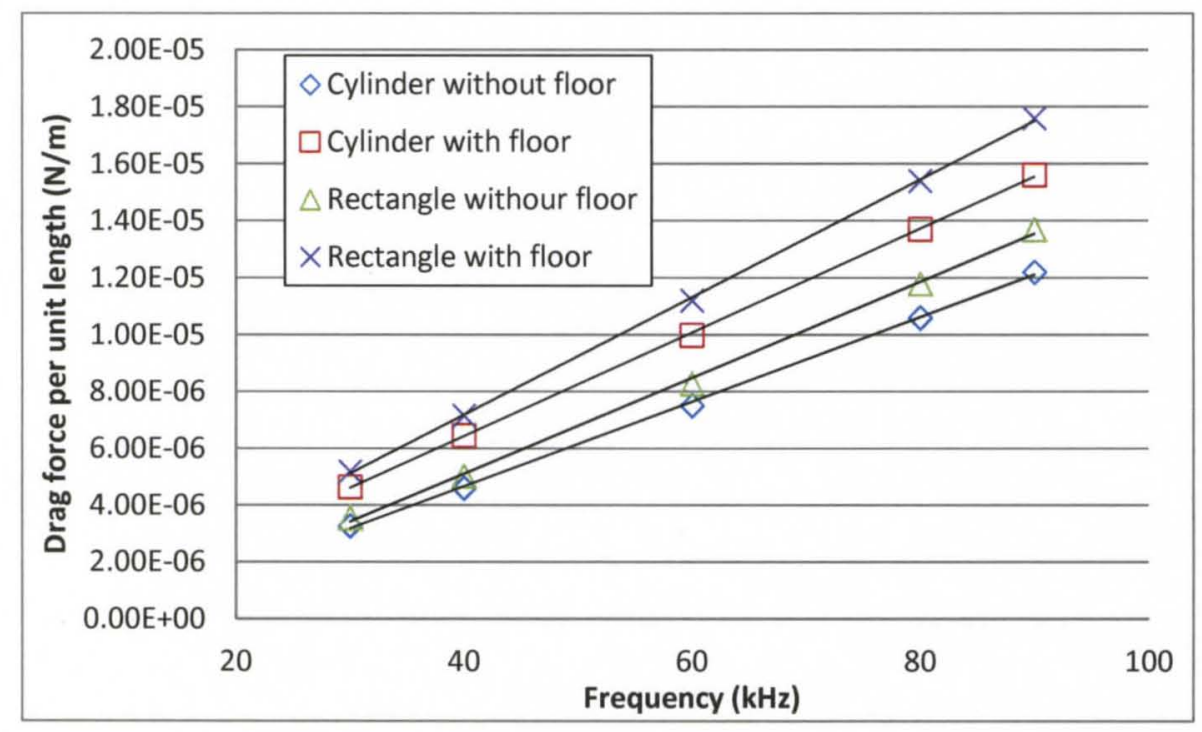

Figure 73. Drag force vs. frequencies for four cases. 
Stokes' equation for calculation of force per unit length acting on the cylinder is listed in Equation 2; In this case, it becomes:

$$
\begin{aligned}
& \frac{d U}{d t}=-S_{0} \omega^{2} \cos (\omega t) ; U=-S_{0} \omega \sin (\omega t) \\
& F_{c y l i n d e r}=-m_{c} k \frac{d U}{d t}-m_{c} k^{\prime} \omega U=m_{c} k S_{0} \omega^{2} \cos (\omega t)+m_{c} k^{\prime} S_{0} \omega^{2} \sin (\omega t)
\end{aligned}
$$

where $\mathrm{u}$ is the velocity and $\mathrm{k}$ and $\mathrm{k}$ ' are the Stokes' model parameters (which also vary with $\omega)$.

\subsubsection{Effect of Shape of Beam Cross Section}

In this section, the effect from the change of beam cross section is considered. In the case of cylinder without floor, the diameter of cylinder is determined by the effective diameter, which makes the area of cylinder the same as that of the rectangle. Therefore, the area of beam cross section is the same in both cases and only the shape changed from cylinder to rectangle. Table 17 lists the ratio of drag forces of cylinder and rectangle. There are two cases: one is without floor; another is with floor. For the first case, the drag forces of the rectangle without floor are divided by those of the cylinder without floor; these are in the range of 1.09-1.12 when frequencies changed from $30 \mathrm{kHz}$ to $90 \mathrm{kHz}$. The same ratio for the cases with the floor vary from 1.11-1.13. It appears that the effect of cross-sectioon is similar for different frequencies, and that the ratio does not vary much due to floor presence. The average of the rate for different cases and different frequencies is 1.11 . This Stokes cylinder model using an effective diameter can represent the force data for a rectangular cross-section for the parameters used here where scaled by 1.11 . 


\begin{tabular}{|l|l|l|l|l|l|}
\hline & \multicolumn{5}{|c|}{ Effect of shape (Rectangle/Cylinder) } \\
\cline { 2 - 6 } & $30 \mathrm{kHz}$ & $40 \mathrm{kHz}$ & $60 \mathrm{kHz}$ & $80 \mathrm{kHz}$ & $90 \mathrm{kHz}$ \\
\hline Cylinder without floor & 1.09 & 1.10 & 1.10 & 1.11 & 1.12 \\
\cline { 1 - 4 } Rectangle without floor & & & & & \\
\hline Cylinder with floor & 1.12 & 1.11 & 1.12 & 1.12 & 1.13 \\
\hline Rectangle with floor & & & & & \\
\hline
\end{tabular}

Table 17. Ratio of drag forces of rectangle and cylinder.

\section{$\underline{3.6 .8 \text { Viscous Effect of Bottom Wall }}$}

Stokes theory considered a cylinder resonating in an infinite fluid. However, the microcantilever beam in this case resonates $2 \mu \mathrm{m}$ above the floor (handle layer). The effect of the floor is studied in this section.

The four cases are divided into two groups: one is cylinder with or without floor; another is rectangle with or without floor. In each group, the shape of beam cross section is same but one involved floor viscosity/boundary effects and the other does not. The rate of drag forces of without and with floor is used to show the effect of the floor. In the cylinder group, the ratio of maximum drag force per unit length decreased from 1.41 to 1.28 as frequency increased from $30 \mathrm{kHz}$ to $90 \mathrm{kHz}$. The rectangle group should a similar ration decrease from 1.46 to 1.28. The ratios are listed in Table 18; the data also are plotted in Figure 74; data is approximately fit by a line but there is some scatter present. However, this data clearly shows the trend that the increase in force due to the floor decreases. 


\begin{tabular}{|l|l|l|l|l|l|}
\hline & \multicolumn{5}{|l|}{ Effect of wall (with floor/without floor) } \\
\cline { 2 - 6 } & $30 \mathrm{kHz}$ & $40 \mathrm{kHz}$ & $60 \mathrm{kHz}$ & $80 \mathrm{kHz}$ & $90 \mathrm{kHz}$ \\
\cline { 2 - 6 } & 1.41 & 1.40 & 1.33 & 1.29 & 1.28 \\
\cline { 1 - 5 } Cylinder without floor & & & & & \\
\hline Rectangle without floor & \multirow{2}{*}{1.46} & 1.42 & 1.35 & 1.31 & 1.28 \\
\hline Rectangle with floor & & & & & \\
\hline
\end{tabular}

Table 18. Ratio of drag forces with floor to those without floor versus frequency

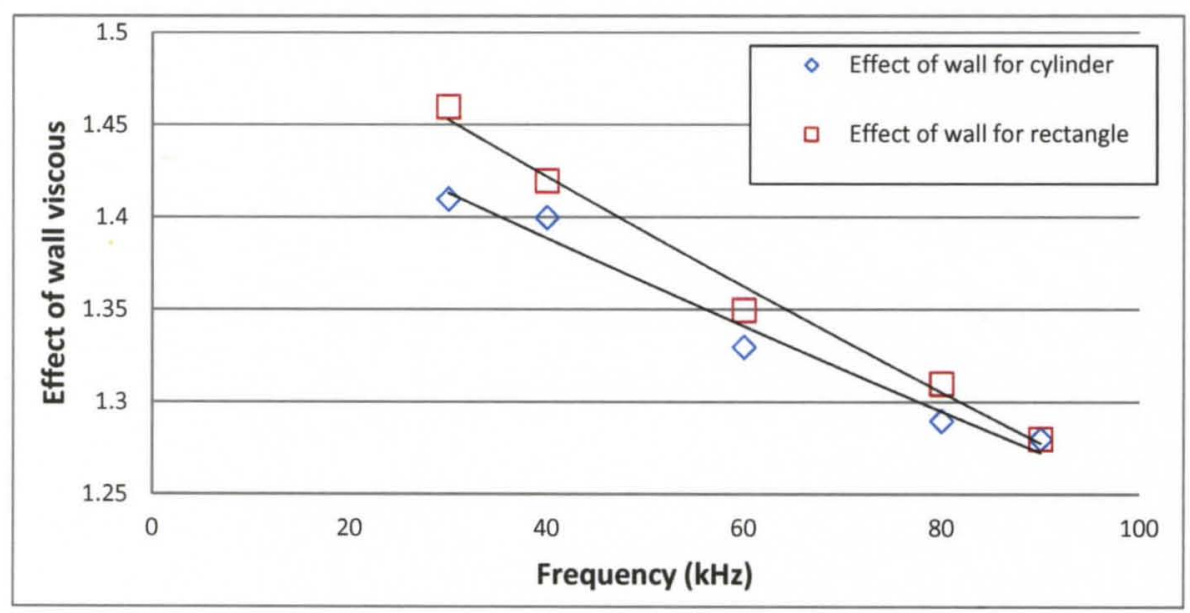

Figure 74. Ratio of drag forces with floor to those without floor.

\subsubsection{Comparison to Stokes' Equation}

From above, the forces of a resonating cylinder without floor were obtained. The values for maximum drag force per unit length in a cycle for different frequencies is listed Table 19. It is clear that the two sets of values are very close. The maximum drag force per unit length in a cycle is also compared with Stokes' equation value for a cylinder without floor which is listed in Table 20 and Table 21. These data are also plotted in Figure 75. In this plot, the data about the ratio of drag force of rectangle with 
floor and cylinder without floor is plotted by trend line. And the trend line, with is expressed by the equation

$$
\mathrm{Y}=20\left(\frac{f}{1000}\right)^{2}-5.4 \frac{f}{1000}+1.7257
$$

where $\omega$ represents the frequency and $\mathrm{Y}$ stands for the ratio of drag forces of rectangle with floor and cylinder without floor.

\begin{tabular}{|l|l|l|l|l|l|}
\hline \multirow{2}{*}{} & \multicolumn{5}{|c|}{ Drag force per unit length $(\mu \mathrm{N} / \mathrm{m})$} \\
\cline { 2 - 6 } & $30 \mathrm{kHz}$ & $40 \mathrm{kHz}$ & $60 \mathrm{kHz}$ & $80 \mathrm{kHz}$ & $90 \mathrm{kHz}$ \\
\hline Stoke equation & 3.31 & 4.62 & 7.52 & 1.07 & 1.22 \\
\hline Cylinder without floor & 3.28 & 4.61 & 7.51 & 1.06 & 1.22 \\
\hline
\end{tabular}

Table 19. Compare Stokes equation and cylinder without floor

\begin{tabular}{|l|l|l|l|l|l|}
\hline & \multicolumn{5}{|c|}{ Compare with theory equation } \\
\cline { 2 - 6 } & $30 \mathrm{kHz}$ & $40 \mathrm{kHz}$ & $60 \mathrm{kHz}$ & $80 \mathrm{kHz}$ & $90 \mathrm{kHz}$ \\
\hline Stoke equation & 1.57 & 1.55 & 1.49 & 1.44 & 1.44 \\
\hline Rectangle with floor & & & & & \\
\hline
\end{tabular}

Table 20. Compare the Stokes equation with rectangle with floor 


\begin{tabular}{|l|l|l|l|l|l|}
\hline & \multicolumn{5}{|l|}{ rectangle with floor vs.cylinder without floor } \\
\cline { 2 - 6 } & $30 \mathrm{kHz}$ & $40 \mathrm{kHz}$ & $60 \mathrm{kHz}$ & $80 \mathrm{kHz}$ & $90 \mathrm{kHz}$ \\
\hline Cylinder without floor & 1.59 & 1.55 & 1.49 & 1.45 & 1.44 \\
\hline Rectangle with floor & & & & & \\
\hline
\end{tabular}

Table 21. Compare the cylinder without floor and rectangle with floor

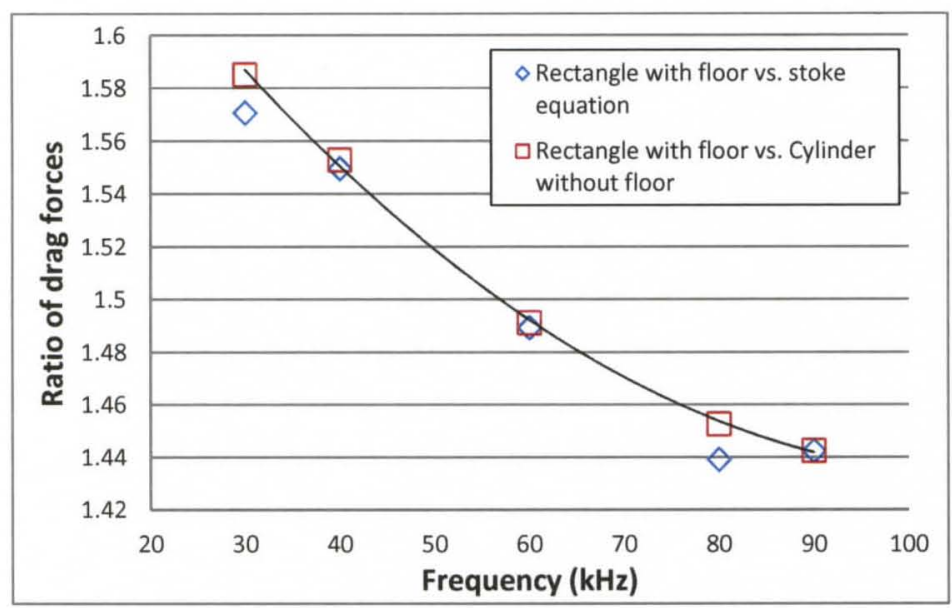

Figure 75. Rectangle with floor compared with Stokes' equation and cylinder without floor.

From this equation, we could tell that there are some relationship between case of rectangle with floor and case of cylinder without floor. The case of cylinder without floor changed to case of rectangle with floor by changing beam cross section and adding the floor viscosity/boundary effect. These two factors lead the results of rectangle with floor to differe from the case discussed in Stokes' theory. Using this model, the drag force for gas sensor can be predicted. First, the Stokes' equation calculats the drag force for cylinder without floor. Second, the equation for $\mathrm{Y}$ is used to calculate the ratio of force in rectangle with floor to cylinder without floor at certain frequency. Third, the ratio is used 
to scale the theory result to get the drag force for rectangle with floor. While the final results will not be an exact match, it should be in the acceptable range.

This force, the drag force of rectangle with floor could be expressed as:

$$
\begin{aligned}
& \frac{d U}{d t}=-S_{0} \omega^{2} \cos (\omega t) ; U=-S_{0} \omega \sin (\omega t) \\
& F_{r e c \tan g / e}=Y\left(-m_{c} k \frac{d U}{d t}-m_{c} k^{\prime} \omega U\right)=Y\left[m_{c} k S_{0} \omega^{2} \cos (\omega t)+m_{c} k^{\prime} S_{0} \omega^{2} \sin (\omega t)\right] \\
& \mathrm{Y}=20\left(\frac{\omega}{2 \pi \cdot 1000}\right)^{2}-5.4 \frac{\omega}{2 \pi \cdot 1000}+1.7257
\end{aligned}
$$

\subsubsection{Effect of Distance to Floor}

When devices are manufactured, the distance to the floor is set by the oxide layer thickness. Since future devices may use something other than $2 \mu \mathrm{m}$, it is important to also understand how this distance $(\delta)$ affects the drag force results. Models were solved using the same set up as the case of cylinder with floor except changing the parameter $\delta$. Figure 76 shows the geometry with mesh for $\delta=0.5,1,4,8$, and 16 . The resonating maximum drag force per unit length is listed in Table 22 and plotted in Figure 77. 
(a)

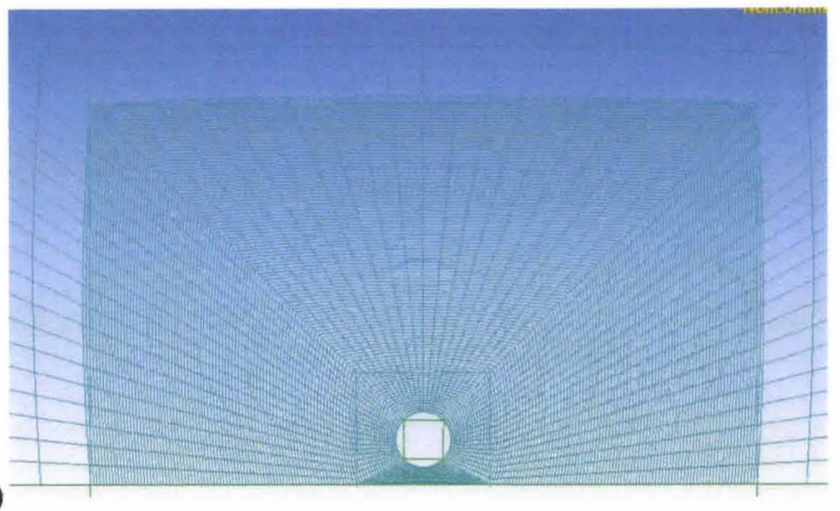

(b)
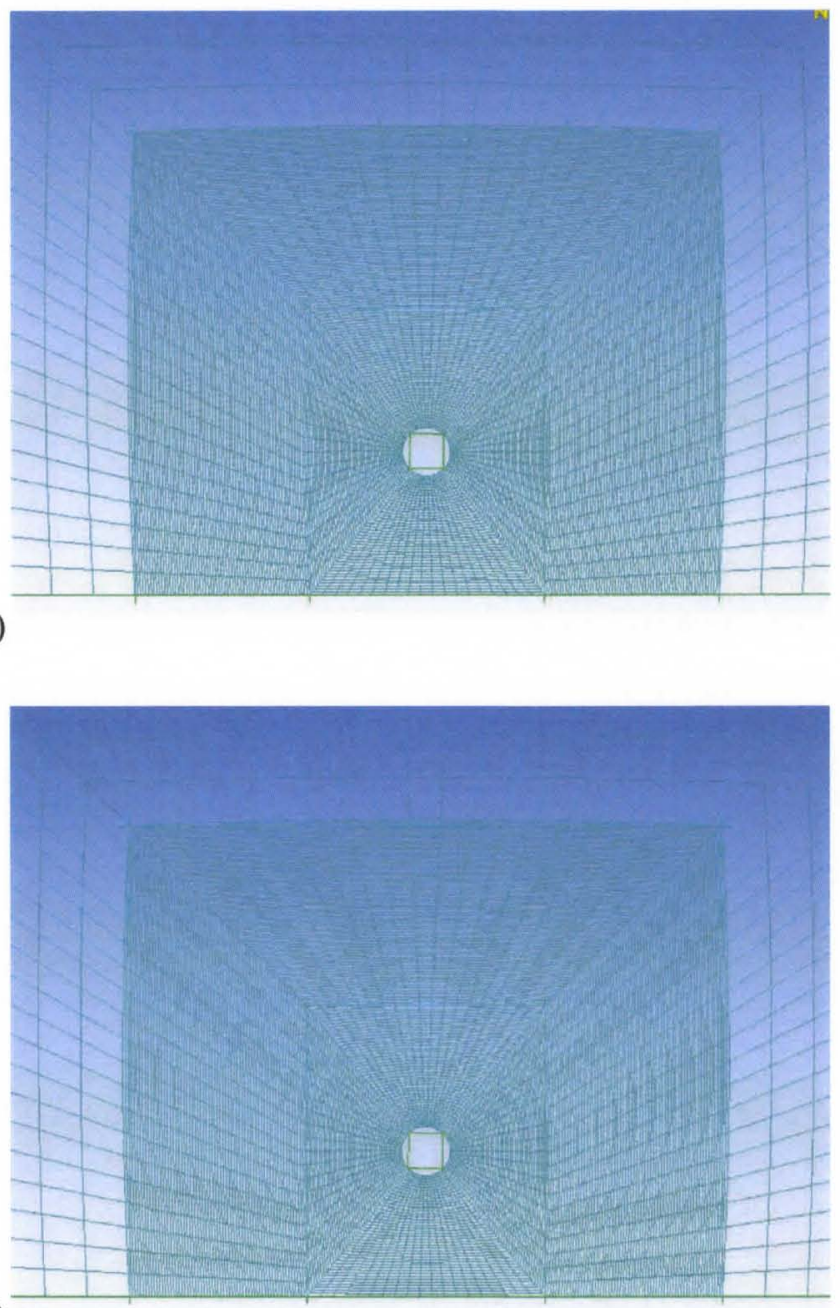

(c) 


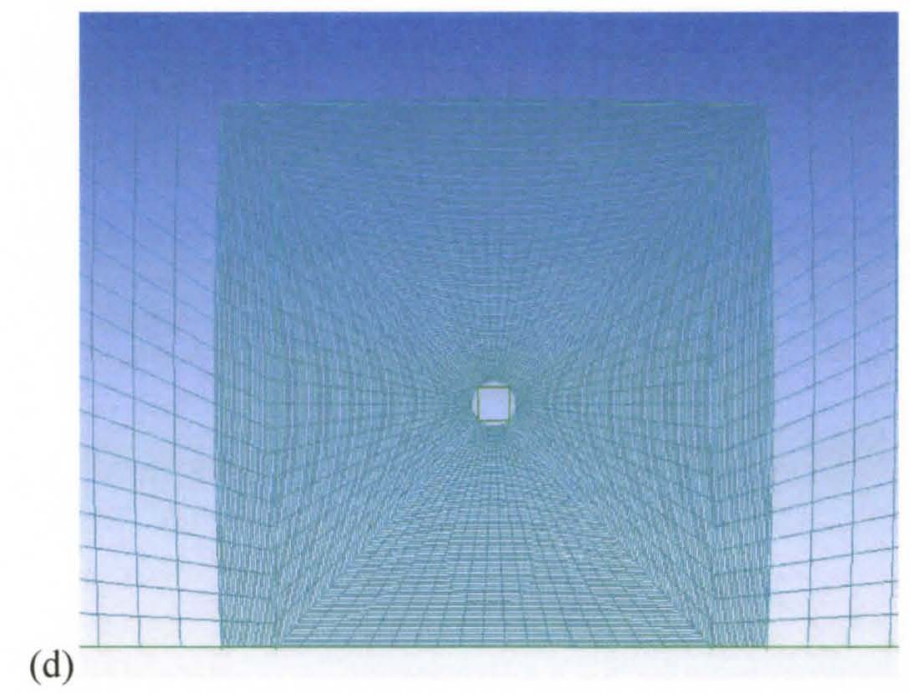

(e)

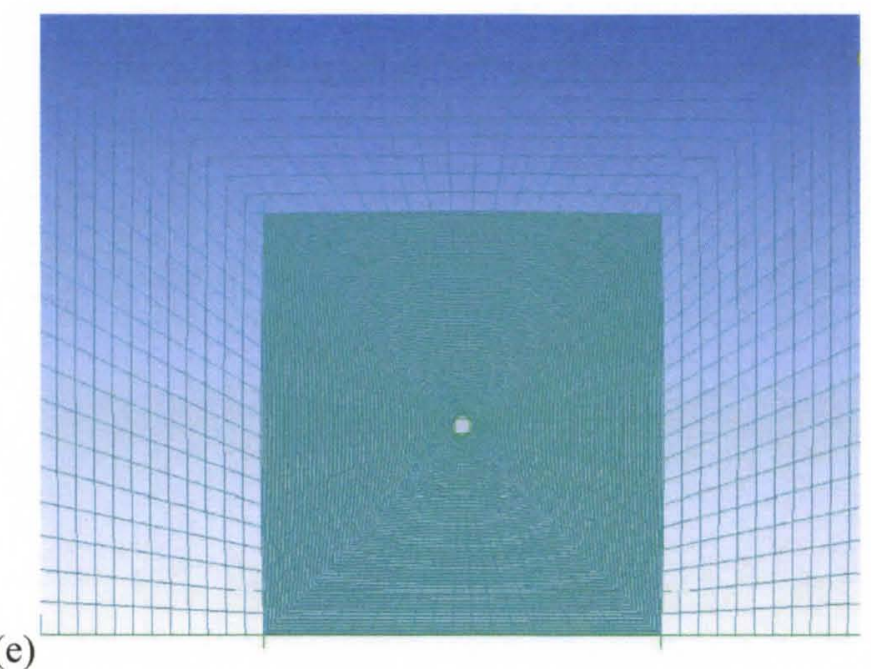

Figure 76. Geometry of cylinder resonating near floor with various values of $\delta$. (a) $\delta=0.5$ $\mu \mathrm{m}$, (b) $1 \mu \mathrm{m}$, (c) $4 \mu \mathrm{m}$, (d) $8 \mu \mathrm{m}$, (e) $16 \mu \mathrm{m}$, where $\delta$ is the gap between the bottom of cylinder and the floor. 


\begin{tabular}{|c|c|}
\hline Elevation from bottom $(\mu \mathrm{m})$ & force per unit length $(\mu \mathrm{N} / \mathrm{m})$ \\
\hline 0.5 & 11.1 \\
\hline 1 & 8.32 \\
\hline 2 & 6.48 \\
\hline 4 & 5.40 \\
\hline 8 & 4.99 \\
\hline 16 & 4.89 \\
\hline
\end{tabular}

Table 22. Drag force of cylinder with various $\delta$ at $40 \mathrm{kHz}$.

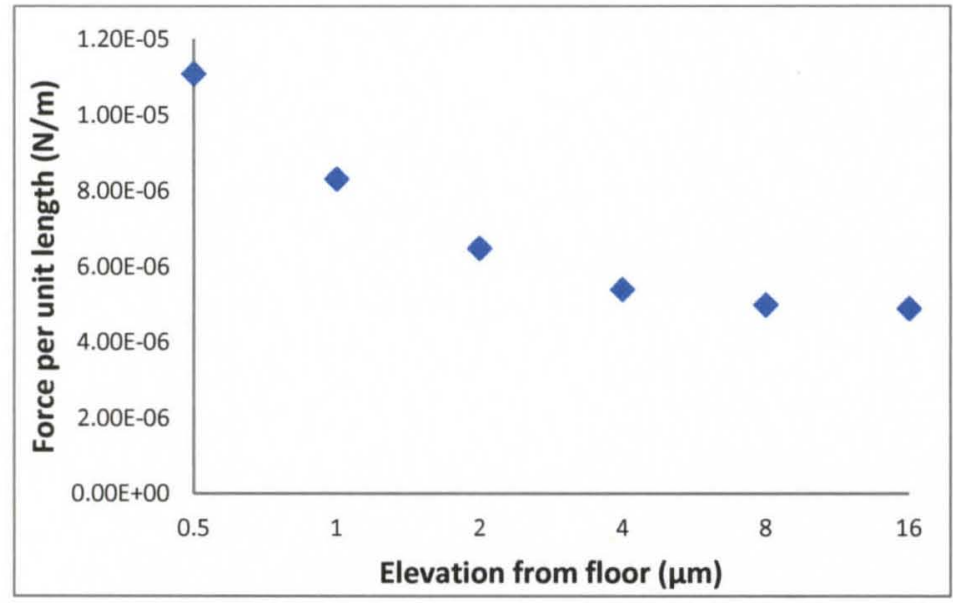

Figure 77. The maximum drag force versus $\delta$ at $40 \mathrm{kHz}$.

Figure 77 shows that when the cylinder is closer to the floor, the floor effects become larger. However as $\delta$ becomes bigger $(>8 \mu \mathrm{m})$, the value of drag force approaches that of the cylinder without a floor $4.61 \mu \mathrm{N} / \mathrm{m}$ as shown in Table 19. In the case of choosing wafer for fabrication, a layer thickness of $\mathrm{SiO}_{2}$ greater than $8 \mu \mathrm{m}$ should largely avoid viscosity/boundary effects due to the floor. 


\subsubsection{Summary of Viscosity Loads on Resonating Beam}

A microcantilever beam resonating in a fluid has been modeled by using SolidWorks, ICEM, and ANSYS CFX. The drag force of cylinder resonating without floor is determined and shown to agree with Stokes' equation. Two effects that differ from the stokes' model but are present in the sensor was evaluated: the shape of the cross-section being rectangular; and the presence of a floor. The cases used different frequencies and found relationship between drag force and frequency. This equation could express the drag force with terms of velocity and acceleration when combined with the Stokes' model. Another approach for model prediction is to simply use CFX directlythe results presented here show that this should lead to accurate values since the analytical model is matched very well. 


\section{CHAPTER 4. ELECTROSTATIC FORCE}

This section will focus on modeling the electrostatic force to better understand the external load acting on the resonating beam system. The goal of this work is to ultimately incorporate these forces into a driven vibration model.

\subsection{Electrostatic Force}

Due to the small scale of micro mechanical systems, the electrostatic force can become relatively large compared with the elastic force of the mechanical structure and the damping force of the surrounding air. It is nonlinear with the distance of separation between the charged surfaces, which may cause severe nonlinearity or instability problems in a vibrating system. [51]

Consider the cantilever beam system resonating about the driving electrode end (as shown in Figure 78 (right)). The model of two parallel plates which are separated by a gap $\mathrm{x}$ was adopted to approximate this system. One plate of the capacitor is fixed and another is movable which is shown in Figure 78 (left). 

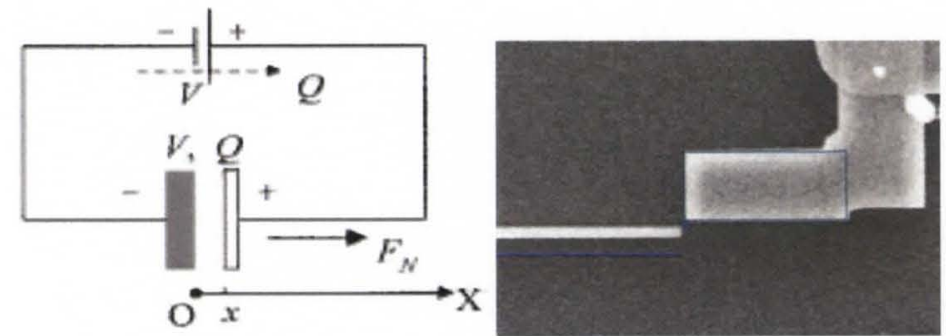

Figure 78. Comparison of parallel-plate actuator (left) to current device at driving electrode (right).[51]

The force applied on the movable plate of the capacitor is

$$
F_{N}=-\frac{\partial E(x)}{\partial x}=-\frac{A \varepsilon \varepsilon_{0}}{2 x^{2}} V^{2}
$$

where $\varepsilon$ the relative permittivity of the medium between two electrodes, which is approximately equal to unity for air and $\varepsilon_{0}$ the permittivity of a vacuum. [52] The model supposes that system is connected with a battery imparting a voltage $\mathrm{V}$. It is clear that electrostatic force related directly with $\mathrm{V}^{2}$ which is equal to the square of the voltage difference between the two plates. Based on Equation (35), the force is attractive since the negative in the equation indicates that $\mathrm{F}_{\mathrm{N}}$ is in the opposite direction as shown.

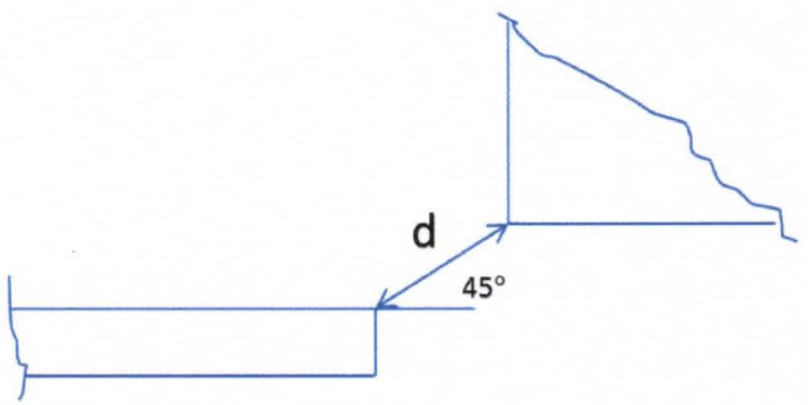

Figure 79. Schematic of beam tip 
This section seeks to approximate the beam tip-driving electrode pair as a parallel plate capacitor. For the device in this study, assume $\mathrm{d}$ is $1.4 \mu \mathrm{m}, \Delta \mathrm{V}$ is $\mathrm{V}_{\text {electrode }}-\mathrm{V}_{\text {beam, }}$, and that the line between corners is $45^{\circ}$ as shown in Figure 79. It is now assumed that the beam-electrode pair acts like a parallel plate separated by gap d of $1.4 \mu \mathrm{m}$ with area $\mathrm{A}=\mathrm{h} \cdot \mathrm{w}$ where $\mathrm{h}$ is the depth normal to the figure and $\mathrm{w}$ is to be determined.
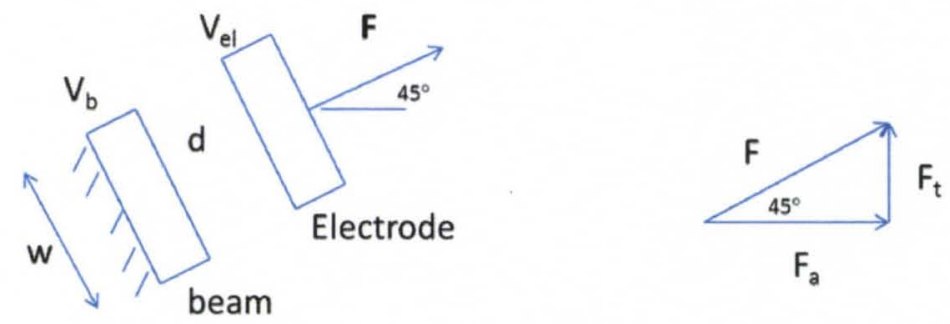

Figure 80 . Schematic of beam-electrode pair and vectorial representation of resulting attractive force.

To maintain $\mathrm{d}$, a force $\mathrm{F}$ must be applied as:

$$
F=\frac{A \varepsilon \varepsilon_{0}}{2 d^{2}}\left(V_{\text {electrode }}-V_{\text {beam }}\right)^{2}
$$

For a gas $\varepsilon_{0} \approx 1$, so this becomes:

$$
F=\frac{w h \varepsilon_{0}}{2}\left(\frac{V_{\text {electrode }}-V_{\text {beam }}}{d}\right)^{2}
$$

The vertical component of $F$ as shown in Figure 80 (Right) is the tangential force $F_{t}$ that is perpendicular to the beam. This is clearly $F_{t}=F / \sqrt{2}$. The other force $F_{a}$ is a load along the beam axis; it does not affect lateral deflection except in large displacement so it could be ignored. 
For cantilever beam, the tip displacement of beam is as:

$$
\delta=\frac{P L^{3}}{3 E I}=\frac{F_{t} L^{3}}{3 E\left(\frac{b^{3} h}{12}\right)}=\left(\frac{4 L^{3}}{E b^{3} h}\right) F_{t}
$$

Now substitute to find

$$
\begin{aligned}
& \delta=\left(\frac{4 L^{3}}{E b^{3} h}\right) \frac{1}{\sqrt{2}}\left(\frac{w h \varepsilon_{0}}{2} \bar{E}^{2}\right) \\
& \bar{E}=\frac{V_{\text {clectrode }}-V_{\text {beam }}}{d}=\text { electric field }
\end{aligned}
$$

After simplifying Equation (39), h is cleared out which means that answer does not depend on beam thickness normal to the vibration plane (thickness of device layer).

$$
\delta=\left(\frac{\sqrt{2} L^{3} w \varepsilon_{0}}{E b^{3}}\right)(\bar{E})^{2}=\left(\frac{\sqrt{2} w \varepsilon_{0}}{E b^{3}}\right)\left(\frac{L}{b}\right)^{3}(\bar{E})^{2}
$$

For $\Delta \mathrm{V}=10 \mathrm{~V}, \bar{E}=\frac{10 \mathrm{~V}}{1.4 \mu \mathrm{m}}$ and $\mathrm{E}, \mathrm{b}, \varepsilon_{0}, \mathrm{~L}$ and $\delta$ are all known; therefore $w$ could be obtained as:

$$
w=\left(\frac{E b^{3}}{\sqrt{2} L^{3} \varepsilon_{0}}\right) \frac{\delta}{(\bar{E})^{2}}
$$

From the ANSYS results (discussed in the next section), $\delta=0.011979 \mu \mathrm{m}$ for $\Delta \mathrm{V}=10 \mathrm{~V}$. Substitution all parameters into Equation (28) leads to w of $2.0112 \mu \mathrm{m}$. This means the beam deflects like a parallel plate capacitor that is $2.0112 \mu \mathrm{m}$ wide with an electrical field is $7.14 \mathrm{~V} / \mu \mathrm{m}$. This value will be compared to the ANSYS results in next section. 


\subsection{Modeling of Electrostatic Beam Tip Forces}

When a voltage difference exists between the beam and the driving electrode, a charge distribution is induced on each surface. These charges lead to electrostatic forces that can cause the beam to deflect. It is conceivable that this deflection itself changes the charge distribution which would then alter the force. Presumably, an equilibrium state would be obtained when the forces due to the beam defection and the surface charges balance each other.

The relationship between beam deflection and the electrostatic forces can be obtained by the solution of coupled field finite element analysis incorporating both mechanical and electrostatic components. A preliminary study has been performed by Dr. Bradshaw using dimensions similar to the beams currently under study. An example of the model and results are shown in Figure 81; the entire beam is modeled (not shown) but only the region near the beam tip and the driving electrode are used in the electrostatic simulation to speed solution time. The deflection observed in the beam tip can be used to estimate an effective load at the beam tip as either a transverse force or a combination of transverse force and tip moment. 

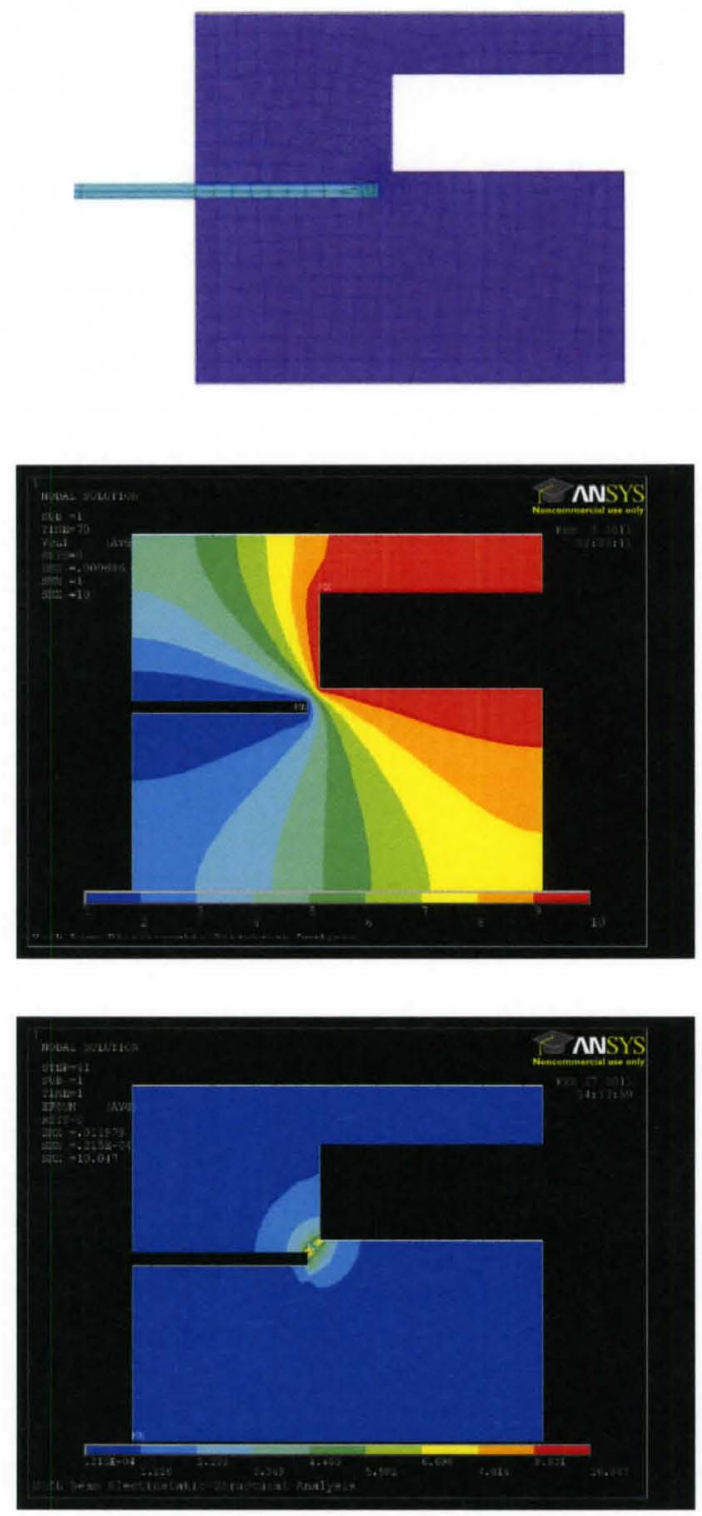

Figure 81. Coupled mechanical-electrostatic model of beam and driving electrode: (top) model of the beam and driving electrode; (middle) voltage contours at $10 \mathrm{~V}$ at driving electrode and beam tip at $0 \mathrm{~V}$; (bottom) associated electrical field. 


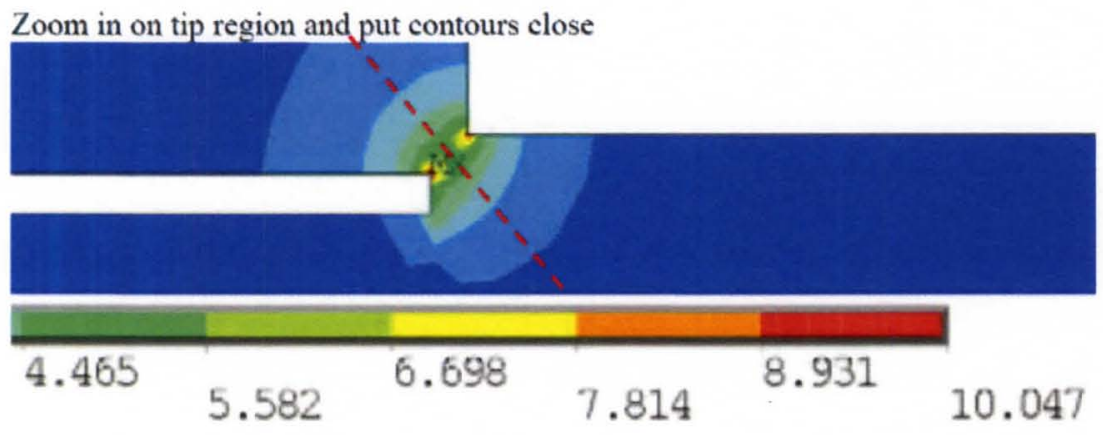

Figure 82. Zoom-in on tip region of associated electrical field.

Figure 82 shows that the electric field in the region of the tip is on the order of 4-7 $\mathrm{v} / \mu \mathrm{m}$. The width of this zone of high electric field is roughly $2-4 \mu \mathrm{m}$ in width. So the displacement results from ANSYS are entirely consistent with those caused for a cantilever beam subjected to a force equal to that from the parallel plate model using an effective width $w=2.011 \mu \mathrm{m}$ divided by $1 / \sqrt{ } 2$.

A simulation was performed in which the driving electrode voltages varied between $+/-10 \mathrm{~V}$ and the response of beam tip was calculated for several beam voltages. As shown in Figure 83, when $\mathrm{V}_{\text {beam }}$ is $0 \mathrm{~V}$, the displacement response is at frequency $2 \mathrm{f}$ (where $\mathrm{f}$ is the driving electrode frequency). For any other voltage, the displacement response is a combination of functions at $\mathrm{f}$ and $2 \mathrm{f}$. 

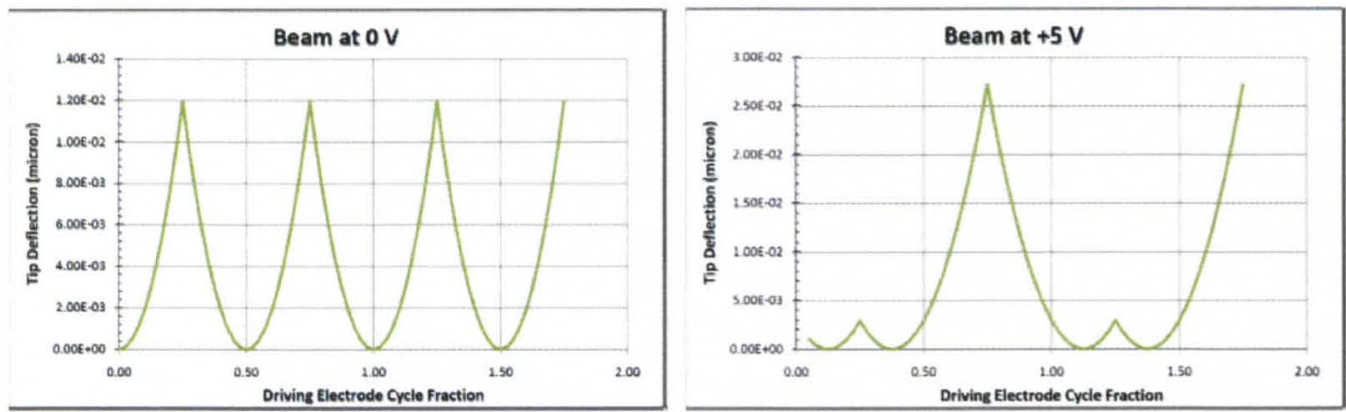

Figure 83. Tip deflection vs. driving electrode cycle fraction. (Left) beam at 0V; (Right) beam at $5 \mathrm{~V}$.
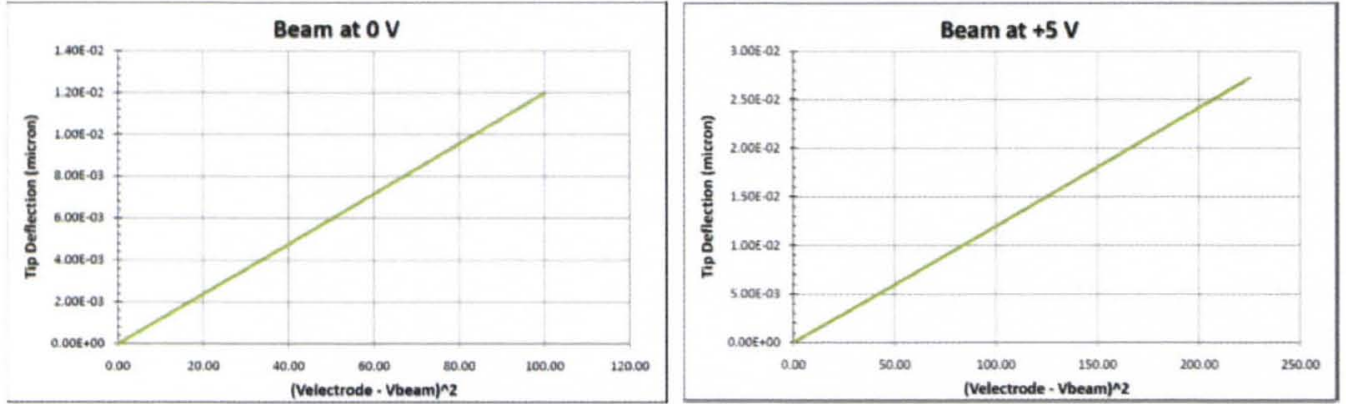

Figure 84. Tip deflection vs. $\Delta \mathrm{V}^{2}$. (Left) beam at $0 \mathrm{~V}$; (Right) beam at $5 \mathrm{~V}$.

When the data from Figure 83 is plotted parametrically as tip displacement versus $(\Delta \mathrm{V})^{2}$ where $\Delta \mathrm{V}$ is the voltage difference $\left(\mathrm{V}_{\text {electrode }}-\mathrm{V}_{\text {beam }}\right)$, a linear relationship is observed. This is shown in Figure 84. Hence, the force approximately follows the same relationship as in parallel plate model (Equation (22)).

The findings thus far indicate that the tip deflection is quite small $(0.0120 \mathrm{~m}$, or $0.0094 \%$ of beam length), indicating that the force available to drive resonance is similarly small $(0.321 \mathrm{nN}$ per beam theory for $\Delta \mathrm{V}=10 \mathrm{~V})$. The parabolic curve shown in Figure 85 shows the relationship between the beam tip displacement and the voltage applied at the driving electrode. 


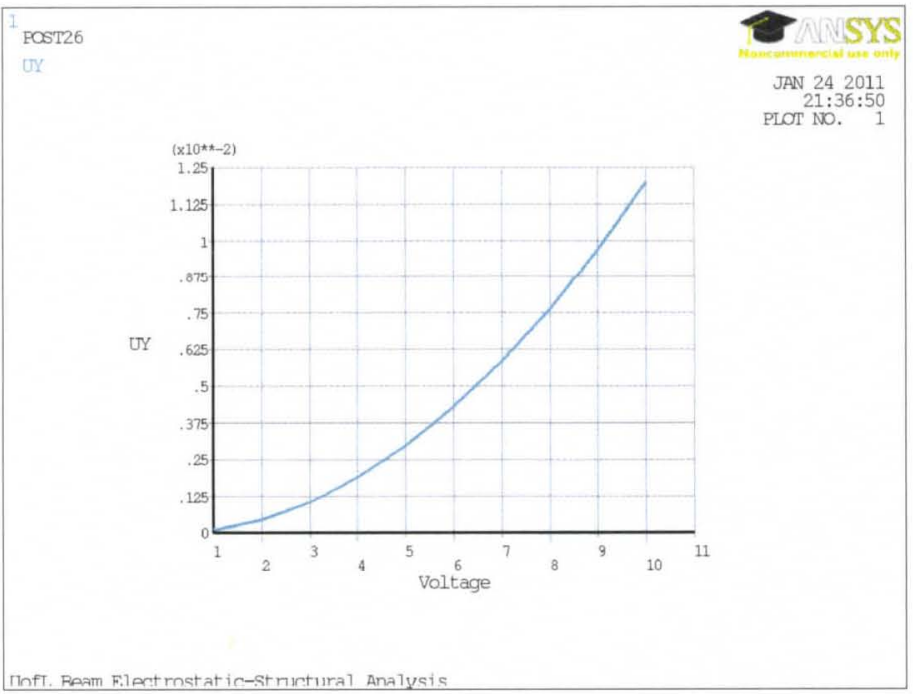

Figure 85 . Beam tip displacement in microns versus voltage applied at the driving electrode when beam is at $0 \mathrm{~V}$.

One interesting feature of Figure 85 is that the same result is obtained if the voltage at the driving electrode is varied from $0 \mathrm{~V}$ to $-10 \mathrm{~V}$ (instead of $+10 \mathrm{~V}$ ). This is because the electrostatic forces are related to the square of the voltage difference; hence the forces are attractive whether the $\Delta \mathrm{V}$ between the driving electrode and beam tip is positive or negative. This relates the odd feature that has existed in the microcantilevers beams studied at the University of Louisville to date, in which they appear to resonate at a driving electrode frequency that is $1 / 2$ of the expected value. For example, the first natural frequency of a beam of a typical size studied in this study can be obtained by the calculation (performed as per unit depth for the beam): 


$$
\begin{aligned}
& f_{1}=\frac{\omega}{2 \pi}=\frac{1}{2 \pi}\left(\frac{1.875}{L}\right)^{2} \sqrt{\frac{E I}{\rho A}} \\
& f_{1}=\frac{1}{2 \pi}\left(\frac{1.875}{128 \cdot 10^{-6} \mathrm{~m}}\right)^{2} \sqrt{\frac{\left(169 \cdot 10^{9} \mathrm{~kg} / \mathrm{ms}^{2}\right)\left(1.1092 \cdot 10^{-25} \mathrm{~m}^{4}\right)}{\left(2.329 \cdot 10^{3} \mathrm{~kg} / \mathrm{m}^{3}\right)\left(1.10 \cdot 10^{-12} \mathrm{~m}^{2}\right)}} \rightarrow f_{1}=92.378 \mathrm{kHz}
\end{aligned}
$$

The microcantilevers beams tend to resonate when the driving electrode signal is in the $40-50 \mathrm{kHz}$ range based on previous studies[42]. Since the electrostatic forces are identical for both a positive and a negative $5 \mathrm{~V}$ voltage difference, the beam tip will experience forces that are at a frequency $2 \mathrm{f}$ if the driving electrode is supplied with an AC signal at frequency $\mathrm{f}$ (as shown in Figure 86).
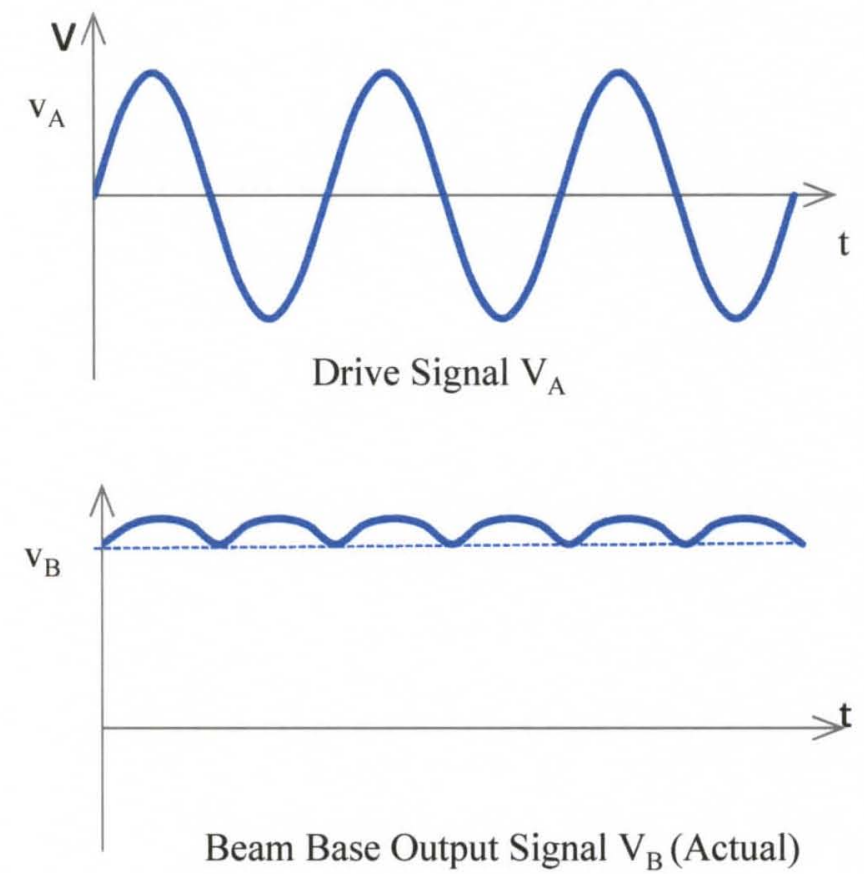

Figure 86. Driving electrode voltage and beam tip load versus time. 
Another complicating factor in device performance is that the loads induced by the electrostatic forces between the driving electrode and the beam tip are likely to be affected by beam tip displacement. For example, as the beam resonates, the beam tip is in a dramatically altered position from the rest state. This new configuration likely has different electrostatic forces than those evident in Figure 86 using beam theory. This dissertation only focuses on beam tip loads in the rest state (i.e. beam not deflected). Incorporation of beam displacement into the tip force causes the model to become nonlinear; analysis of such effects is beyond the scope of this dissertation.

\subsection{Driving Electrode Force Model}

From ANSYS the relationship between the tip displacement $\delta_{0}$ for a given voltage difference $\Delta \mathrm{V}_{0}$ could be as:

$$
\delta=K \cdot \Delta V^{2}, o r, K=\frac{\delta_{0}}{(\Delta V)^{2}}
$$

This could becomes

$$
\delta=\delta_{0}\left(\frac{\Delta V}{\Delta V}\right)^{2}
$$

Since it is a cantilever beam, we can determine the force applied to the beam to be

$$
\left.\begin{array}{l}
F=\frac{3 E I}{L^{3}} \delta=\left(\frac{E h}{4}\right)\left(\frac{b}{L}\right)^{3} \delta \\
F_{0}=\frac{3 E I}{L^{3}} \delta=\left(\frac{E h}{4}\right)\left(\frac{b}{L}\right)^{3} \delta_{0}
\end{array}\right\} \Rightarrow \frac{F}{F_{0}}=\frac{\delta}{\delta_{0}}
$$


Because the force could be express as $F=\left(\frac{F}{F_{0}}\right) F_{0}$, according Equation above, $\mathrm{F}$ then becomes

$$
F=\left(\frac{\Delta V}{\Delta V}\right)^{2}\left(\frac{E h}{4}\right)\left(\frac{b}{L}\right)^{3} \delta_{0}=K\left(\frac{E h}{4}\right)\left(\frac{b}{L}\right)^{3} \Delta V^{2}
$$

If defined $\mathrm{k}=K\left(\frac{E h}{4}\right)\left(\frac{b}{L}\right)^{3}$ which is constant, and $\mathrm{F}=\mathrm{k} \cdot \Delta \mathrm{V}^{2}$. In experiment, the voltage varies at the driving electrode in a sinusoidal manner while the beam voltage is maintained at a constant value. Hence $\Delta V=\left(V_{E} \cdot \sin (\omega t)+V_{0}\right)-V_{B}$, where $V_{E}$ is the peak driving electrode voltage and $V_{B}$ is the beam voltage. $V_{0}$ is DC voltage offset. If square this term and make an expansion,

$$
\Delta V^{2}=\left(V_{0}-V_{B}\right)^{2}+2 V_{E}\left(V_{0}-V_{B}\right) \sin (\omega t)+\left(V_{E}\right)^{2} \sin (\omega t)^{2}
$$

Rearrange above equation,

$$
\begin{aligned}
& \Delta V^{2}=h_{0}+h_{1} \sin (\omega t)-h_{2} \cos (2 \omega t) \\
& h_{0}=\left(V_{0}-V_{B}\right)^{2}+\frac{1}{2}\left(V_{E}\right)^{2} \\
& h_{2}=\left(\frac{1}{2}\right)\left(V_{E}\right)^{2}
\end{aligned}
$$



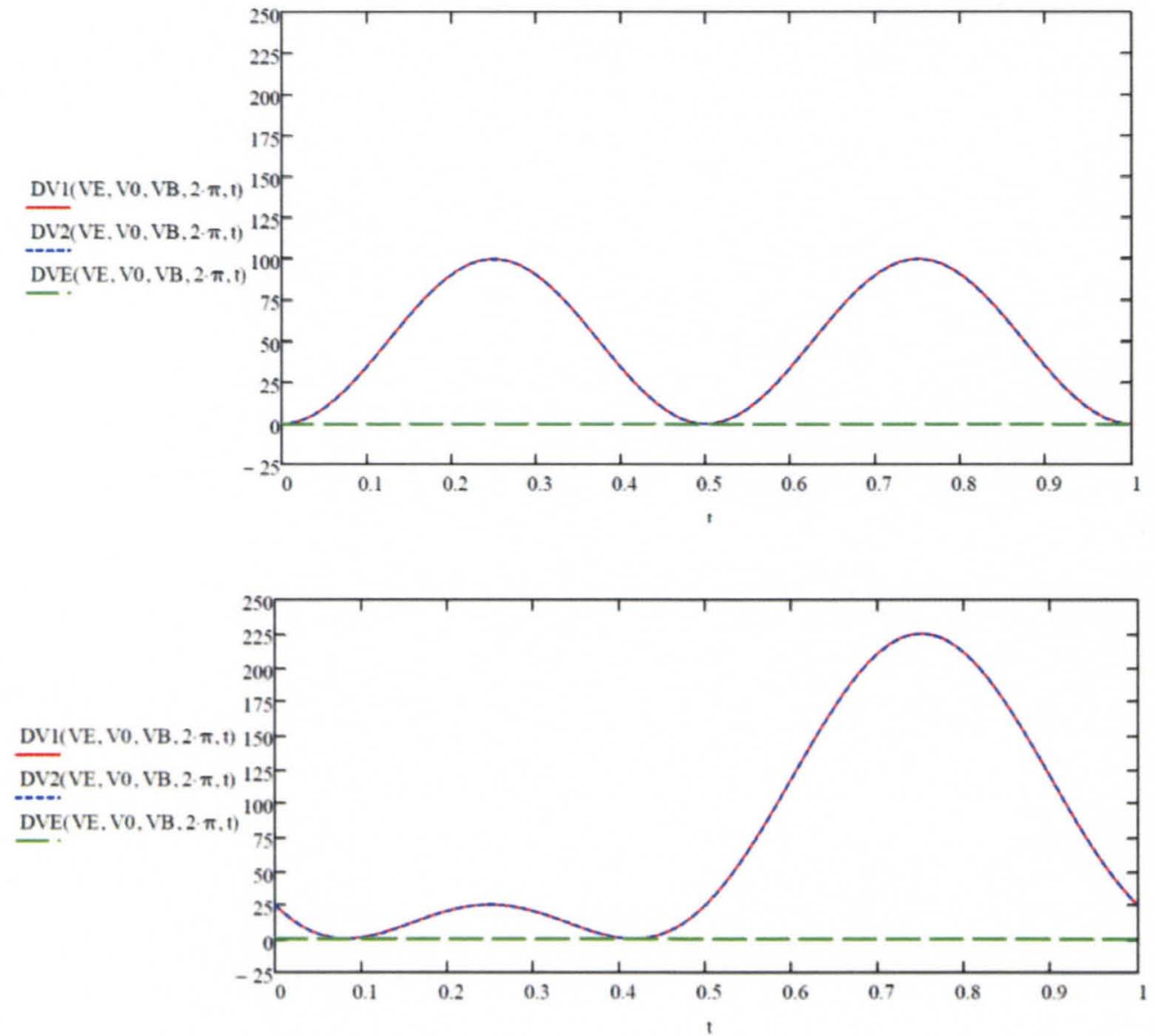

Figure 87. $\Delta \mathrm{V}^{2}$ versus time. $(\mathrm{Up}) \mathrm{V}_{\mathrm{E}}=10 \mathrm{~V}, \mathrm{~V}_{\mathrm{B}}=0 \mathrm{~V}, \mathrm{~V}_{0}=0 \mathrm{~V}$; (Bottom) $\mathrm{V}_{\mathrm{E}}=10 \mathrm{~V} \mathrm{~V}$ $=5 \mathrm{~V}, \mathrm{~V}_{0}=0 \mathrm{~V}$

Figure 87 which generated by Dr. Bradshaw shows that it is a single cycle for different voltage differences. This matches what we found in ANSYS simulation.

According the relationship between force and voltage difference $\mathrm{F}=\mathrm{k} \cdot \Delta \mathrm{V}^{2}$, the electrostatic force could be as:

$$
F=k h_{0}+k h_{1} \sin (\omega t)-k h_{2} \cos (2 \omega t)
$$




\subsection{Conclusion}

The microcantilever beam has been assessed using a finite element model that combines both electrostatics and structural analysis. The driving electrode at $10 \mathrm{~V}$ leads to relatively small tip displacements and therefore corresponds to relatively small tip loads. It is not surprising that resonance in gas may be difficult to achieve as the energy input per cycle is small; if damping effects remove more energy than is gained per cycle, resonance cannot be achieved. It may be that alternate forms to induce resonance could lead to greater energy input per cycle and achieve resonance more easily in the presence of gas. The electrostatic modeling also provides a way to explain the unusual result found that some beams resonate at roughly half of the frequency at which resonance would be expected based on beam vibration theory. The nature of the electrostatic loading between the beam tip and the driving electrode leads to forces which cause beam resonance at frequencies equal to roughly half the expected resonance frequency. This explanation is based on an assumption that the beam and the driving electrode are connected by a battery. New silver glue provides a way to connect the handle layer to the ground. In this case, the beam and the driving electrode are two parts separately. And the electrostatic force acting on the beam tip has the same frequency as the driving signal. 


\section{CHAPTER 5. VIBRATION MODELING}

The final step of this research focuses on modeling to better understand the overall behavior of the resonating beam system. This begins with the derivation of the undamped and damped free vibration of a cantilever beam into mode shapes and associated sinusoidal natural frequencies. This model is then extended to describe vibration and the system behavior due to electrostatic forces are investigated. The goal of this section will be to relate the predicted magnitude of frequency shift and/or resonance curve shape change to the properties of the gas in which it is operating utilizing the assumptions described in earlier chapters.

\subsection{Model and Governing Equation of Microcantilever Beam Resonation}

Resonance of the microcantilever beam is caused by a sinusoidal voltage signal at the drive electrode; this leads to repetitive electrostatic forces (have been discussed in Chapter 4). For known interaction forces, the dynamics of the microcantilever beam can be accurately described by a fourth order partial differential equation:

$$
E I \frac{\partial^{4} u}{\partial x^{4}}+m_{B} \frac{\partial^{2} u}{\partial t^{2}}=f_{g}(x, t)+f_{d}(x, t)
$$

where $u$ is the beam lateral displacement, $x$ is the position along the beam length, $t$ is time, $\mathrm{E}$ is the Young's modulus of the beam, $\mathrm{I}$ is the moment of inertia of the beam, $\mathrm{m}_{\mathrm{B}}$ 
is the beam mass per unit length, $f_{d}(x, t)$ is the distributed load per unit length and $f_{g}(x, t)$ is the fluid viscous per unit length, which accounts for the effect of gas damping.

The gas dynamic load $f_{g}(x, t)$ can be determined by

$$
f_{g}(x, t)=-c_{g} \frac{\partial u}{\partial t}-m_{g} \frac{\partial^{2} u}{\partial^{2} t}
$$

where $c_{g}$ and $m_{g}$ are the effective damping and additive mass from the gas per unit length, respectively, as derived in Chapter 3 for a rectangle cross-section beam and resonating above a floor. As the equation above shows, the fluid load has two components: one is inphase with the velocity, $c_{g} \partial u / \partial t$, which is denominated by viscous damping; and the other is in-phase with the acceleration, $m_{g} \partial^{2} u / \partial^{2} t$, which is denominated by added mass that effectively "clings" to the beam as it resonates. The viscous coefficient $c_{g}$ affects the quality factor $\mathrm{Q}$ and shifts the peak resonant frequency of the beam, while $m_{g}$ only produces a small shift in the resonance frequency of the beam. [53] Substituting Equation (51)into Equation (50), and the beam governing equation is rearranged as:

$$
\left(m_{B}+m_{g}\right) \frac{\partial^{2} u}{\partial t^{2}}+c_{g} \frac{\partial u}{\partial t}+E I \frac{\partial^{4} u}{\partial x^{4}}=f_{d}(x, t)
$$

The boundary value problem presented above can then be solved numerically. Based on methods using by several textbooks, the above equation is solved in the next section.[5455] 


\subsubsection{Free Vibration of The Beam}

Begin first with the case of free vibration, in which $f_{d}(x, t)=0$, the differential equation of motion reduces to

$$
\frac{\partial^{2} u}{\partial t^{2}}+a^{2} \frac{\partial u}{\partial t}+d^{2} \frac{\partial^{4} u}{\partial x^{4}}=0
$$

where

$$
a^{2}=\frac{c_{g}}{\left(m_{B}+m_{g}\right)}, d^{2}=\frac{E I}{\left(m_{B}+m_{g}\right)}
$$

Using the method of separation of variables, we assume a solution of the form

$$
u(x, t)=\phi(x) q(t)
$$

where $\phi(x)$ is a function which depends only on space and $q(t)$ depends only on time. Substituting Equation (53)into Equation (55), we obtain

$$
\phi \frac{d^{2} q}{d t^{2}}+a^{2} \phi \frac{d q}{d t}+d^{2} q \frac{d^{4} \phi}{d x^{4}}=0
$$

Divide through by $\phi q$ and pull all terms with time dependence to the right hand side leads to:

$$
d^{2} \frac{1}{\phi} \frac{d^{4} \phi}{d x^{4}}=\frac{-1}{q} \frac{d^{2} q}{d t^{2}}-a^{2} \frac{1}{q} \frac{d q}{d t}
$$


Since the left hand side is a function of $x$, and the right hand side is a function of time, the equation can only be true if both sides are equal to a constant to be determined. Calling this value $\omega_{n}{ }^{2}$, Equation (57) can be rewritten as:

$$
\frac{d^{4} \phi}{d x^{4}}-\beta^{4} \phi=0
$$

and

$$
\frac{d^{2} q}{d t^{2}}+a^{2} \frac{d q}{d t}+\omega_{n}^{2} q=0
$$

where

$$
\beta^{4}=\frac{\omega_{n}^{2}}{d^{2}}=\frac{\omega_{n}{ }^{2}\left(m_{B}+m_{g}\right)}{E I}
$$

The general solutions of Equation (59) and (60) are given as

$$
\phi(x)=c_{1} \sin (\beta x)+c_{2} \cos (\beta x)+c_{3} \sinh (\beta x)+c_{4} \cosh (\beta x)
$$

and

$$
q_{h}=e^{-a^{2} t / 2}\left(c_{5} \cos \omega_{d} t+c_{6} \sin \omega_{d} t\right)
$$

\subsubsection{Constraint At Beam Base}

The base arrangement of the actual microcantilever beam system is quite complicated, consisting of either 2 support legs in a tee configuration (see Figure 2 ) or a staggered leg arrangement (see Figure 2 ). Due to this arrangement, there is clearly a 
finite stiffness present at the beam base since without deformation of the support legs no signal would be measured. However, this work assumes that the beam base is fixed, allowing neither rotation or translation. One important question is whether this assumption is valid; if so, how much difference would it make to use an alternate arrangement that incorporates base stiffness into the model?

The beam base resistance finite element model presented earlier (see Figure 19 and Figure 20) contains the fully stiffness behavior of both the beam and the support base. During that solution, the model also calculates the $1^{\text {st }}$ and $2^{\text {nd }}$ natural frequency of the beam. Variations in the $1^{\text {st }}$ natural frequency provide a measure of the effect that beam base stiffness is likely to have in the resonant frequencies of the beam as fabricated. According to the model, the natural frequencies do change somewhat with different base configurations; this is demonstrated in Figure 88 below. These results indicate that variation of $1^{\text {st }}$ natural frequency on the order of $1-3 \%$ occurs due to beam base configuration. In this work, the effect of beam base stiffness will be neglected and the beam is modeled as fixed at the base. The effect of beam base stiffness could be included in future vibration models. 


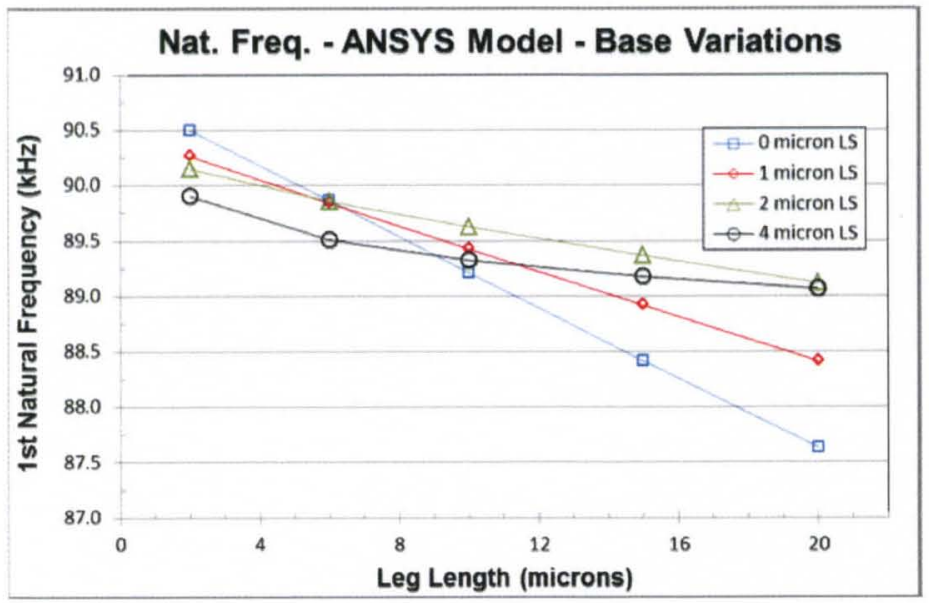

Figure 88 . Variation in $1^{\text {st }}$ natural frequency for asymmetric beam base arrangement with various leg separation and leg length values.

\subsubsection{Mode Shapes of Cantilever Beam}

For a cantilever beam with length $\mathrm{L}$, fixed at $\mathrm{x}=0$ and free at end $\mathrm{x}=\mathrm{L}$, the boundary conditions are:

1. The displacement and the slope of the beam at the fixed end are zero, and

2. The moment and the shear force on the beam at the free end are zero.

These conditions can be written in mathematical form as:

$$
u(0, t)=\frac{\partial u}{\partial x}(0, t)=\frac{\partial^{2} u}{\partial x^{2}}(L, t)=\frac{\partial^{3} u}{\partial x^{3}}(L, t)=0
$$

Equation (63) implies that:

$$
\phi(0)=\frac{\partial \phi}{\partial x}(0)=\frac{\partial^{2} \phi}{\partial x^{2}}(L)=\frac{\partial^{3} \phi}{\partial x^{3}}(L)=0
$$

Differentiating Equation (61) three times with respect to x leads to: 


$$
\begin{gathered}
\frac{d \phi}{d x}=\beta\left(c_{1} \cos (\beta x)-c_{2} \sin (\beta x)+c_{3} \cosh (\beta x)+c_{4} \sinh (\beta x)\right) \\
\frac{d^{2} \phi}{d x^{2}}=\beta^{2}\left(-c_{1} \sin (\beta x)-c_{2} \cos (\beta x)+c_{3} \sinh (\beta x)+c_{4} \cosh (\beta x)\right) \\
\frac{d^{3} \phi}{d x^{3}}=\beta^{3}\left(-c_{1} \cos (\beta x)+c_{2} \sin (\beta x)+c_{3} \cosh (\beta x)+c_{4} \sinh (\beta x)\right)
\end{gathered}
$$

Substituting Equation (64) into Equations. (61), (65),(66) and (67) leads to:

$$
\begin{gathered}
c_{2}+c_{4}=0 \\
c_{1}+c_{3}=0 \\
-c_{1} \sin (\beta L)-c_{2} \cos (\beta L)-c_{1} \sinh (\beta L)-c_{2} \cosh (\beta L)=0 \\
-c_{1} \cos (\beta L)+c_{2} \sin (\beta L)-c_{1} \cosh (\beta L)-c_{2} \sinh (\beta L)=0
\end{gathered}
$$

Equation (68) and (69) require $c_{2}=-c_{1}$ and $c_{4}=-c_{2}$, leaving only two unknowns $\left(c_{1}\right.$, $\mathrm{c}_{2}$ ) to be determined. Equation (70) and (71) can be written in matrix form as

$$
\left[\begin{array}{ll}
a_{11} & a_{12} \\
a_{21} & a_{22}
\end{array}\right]\left[\begin{array}{l}
c_{1} \\
c_{2}
\end{array}\right]=\left[\begin{array}{l}
0 \\
0
\end{array}\right]
$$

where $a_{11}, a_{12}, a_{21}, a_{22}$ are given as

$$
a_{11}=\sin (\beta L)+\sinh (\beta L)
$$




$$
\begin{aligned}
& a_{12}=\cos (\beta L)+\cosh (\beta L) \\
& a_{21}=\cos (\beta L)+\cosh (\beta L) \\
& a_{22}=-\sin (\beta L)+\sinh (\beta L)
\end{aligned}
$$

For nontrivial solution, the determinant of the matrix in Equation (72) must be 0 . This leads to

$$
a_{11} a_{22}-a_{12} a_{21}=0
$$

which is only satisfied by:

$$
\cos (\beta L) \cosh (\beta L)+1=0
$$

This is a nonlinear equation which has an infinite number of roots $\beta \mathrm{L}$ with which satisfy the first 4 being:

$$
\beta L=1.8751,4.6941,7.8548,10.9955
$$

For the rectangular beam considered here, $I=h b^{3} / 12$ and $m_{B}=\rho b h$, where $\mathrm{b}$ is the beam width in plane of vibration, $h$ is the beam height normal to the plane of vibration thickness of device layer, and $\rho$ is the density of the beam material. Using Equation (60)and the expressions for $\mathrm{I}$ and $\mathrm{m}$ leads to:

$$
\omega_{n}^{2}=\beta^{4} \frac{E I}{m_{B}+m_{g}}=\beta^{4} \frac{E h b^{3}}{12\left(m_{B}+m_{g}\right)}
$$




$$
\omega_{n}=\beta^{2} \sqrt{\frac{E I}{m_{B}+m_{g}}}=\frac{(\beta L)^{2}}{\sqrt{12}} \frac{b}{L^{2}} \sqrt{\frac{E b h}{m_{B}+m_{g}}}
$$

This can be compared with the frequency of free vibration of a cantilever beam without gas and damping:

$$
\omega_{0}=\beta^{2} \sqrt{\frac{E I}{m_{B}}}=\frac{(\beta L)^{2}}{\sqrt{12}} \frac{b}{L^{2}} \sqrt{\frac{E b h}{\rho b h}}=\frac{(\beta L)^{2}}{\sqrt{12}} \frac{b}{L^{2}} \sqrt{\frac{E}{\rho}}
$$

Hence, it is clear that $\omega_{\mathrm{n}}$ in Equation (81) is identical to the natural frequency of an undamped beam in which the beam mass per unit length has been augmented by an additional mass $m_{g}$ due to the gas (i.e. with $m_{B}$ replaced by $m_{B}+m_{g}$ ). If the gas causes no damping of the beam (i.e. $c_{g}=0$ ), the value $\omega_{n}$ will represent a natural frequency of the resonating cantilever beam. For systems in which detection is accomplished by mass adsorption onto the surface of the beam, this added mass is source of the frequency shift. For the system considered in this dissertation, the gas is damping the beam (i.e. $c_{g} \neq 0$ ) and its presence leads to an inertial load component that can be considered as an additive mass $m_{g}$ traveling with beam (but not adsorbed to the beam surface). In both cases, the value $\omega_{n}$ then represents the undamped natural frequency $\left(\omega_{0}\right)$. The damped natural frequency $\omega_{\mathrm{d}}$ is calculated in terms of this value using Equation (79); if the damping parameter $\mathrm{a}^{2}$ is non-zero, the value $\omega_{\mathrm{d}}$ will be less than $\omega_{\mathrm{n}}$ from Equation (81). Using Equation (70), the ratio of $c_{2}$ to $c_{1}$ can be written as 


$$
\frac{c_{2}}{c_{1}}=-\frac{\sin (\beta L)+\sinh (\beta L)}{\cos (\beta L)+\cosh (\beta L)}
$$

Substituting $c_{1}=-c_{3}$ and $c_{4}=-c_{2}$ into Eq. (71) leads to:

$$
\phi(x)=c_{1}\left\{\left(\sin (\beta x)-\sinh (\beta x)+\frac{c_{2}}{c_{1}}[\cos (\beta x)-\cosh (\beta x)]\right\}\right.
$$

Each different value of $\beta$ leads to a different form of $\phi(x)$; there are known as the mode shape of the resonating microcantilever beam.

\subsubsection{Properties of The Mode Shapes}

Now represent the different mode shapes as $\phi_{j}(x), \mathrm{j}=1, \ldots, \infty$. Where $\mathrm{j}=1$

corresponds to $\beta \mathrm{L}=1.8751, \mathrm{j}=2$ corresponds to $\beta \mathrm{L}=4.694$, etc. Note that these mode shapes satisfy Equation (55), or:

$$
\frac{d^{4} \phi_{j}(x)}{d x^{4}}=\beta_{j}^{4} \phi_{j}(x)
$$

Multiplying both sides by $\phi_{k}(x)$ and integrating from 0 to $\mathrm{L}$ leads to:

$$
\begin{aligned}
& \int_{0}^{L} \beta_{j}^{4} \phi_{j}(x) \phi_{k}(x) d x=\int_{0}^{L} \frac{d^{4} \phi_{j}(x)}{d x^{4}} \phi_{k}(x) d x \\
& \int_{0}^{L} \frac{d^{2} \phi_{j}(x)}{d x^{2}} \frac{d^{2} \phi_{k}(x)}{d x^{2}} d x+\left.\frac{d^{3} \phi_{j}(x)}{d x^{3}} \phi_{k}(x)\right|_{0} ^{L}-\left.\frac{d^{2} \phi_{j}(x)}{d x^{2}} \frac{d \phi_{k}(x)}{d x}\right|_{0} ^{L}
\end{aligned}
$$

Using the boundary conditions of Equation (63) and (64), Equation (86) reduces to: 


$$
\int_{0}^{L} \beta_{j}^{4} \phi_{j}(x) \phi_{k}(x) d x=\int_{0}^{L} \frac{d^{2} \phi_{j}(x)}{d x^{2}} \frac{d^{2} \phi_{k}(x)}{d x^{2}} d x
$$

Switching the role of $\phi_{j}(x)$ and $\phi_{k}(x)$ leads to a related equation:

$$
\int_{0}^{L} \beta_{k}^{4} \phi_{k}(x) \phi_{l}(x) d x=\int_{0}^{L} \frac{d^{2} \phi_{k}(x)}{d x^{2}} \frac{d^{2} \phi_{j}(x)}{d x^{2}} d x
$$

Subtraction of Equation (88) from Equation (87) leads to

$$
\left(\beta_{j}^{4}-\beta_{k}^{4}\right) \int_{0}^{L} \phi_{j}(x) \phi_{k}(x) d x=0
$$

From this, it is clear that if $\mathrm{j} \neq \mathrm{k}$ that

$$
\int_{0}^{L} \phi_{j}(x) \phi_{k}(x) d x=0
$$

and from Equation (88) that:

$$
\int_{0}^{L} \frac{d^{2} \phi_{k}(x)}{d x^{2}} \frac{d^{2} \phi_{j}(x)}{d x^{2}} d x=0
$$

For $\mathrm{j}=\mathrm{k}, \int_{0}^{L} \phi_{j} \phi_{k} d x=\int_{0}^{L}\left(\phi_{j}(x)\right)^{2} d x$ is an arbitrary constant. If $\mathrm{c}_{1}$ in Equation (84) is selected such that

$$
\int_{0}^{L}\left(\phi_{j}(x)\right)^{2} d x=1
$$

the first four roots of $c_{1}$ are then obtained as: 


$$
c_{1}=64.8857,90.0204,88.3191,88.3918
$$

These values of $c_{1}$ satisfies Equation (84) and the associated mode shapes $\phi_{\mathrm{j}}(\mathrm{x}), \mathrm{j}=1, \ldots$, $\infty$, are referred to as the normalized mode shapes.

\subsubsection{Forced Resonance: $F=F_{0} \cdot \sin (\omega \cdot t)$}

Of particular interest to this dissertation is the care of resonance induced by timevarying end load. For the case of general distributed loading along the beam by $f(x, t)$, the governing differential equation becomes:

$$
\frac{\partial^{2} u}{\partial t^{2}}+a^{2} \frac{\partial u}{\partial t}+d^{2} \frac{\partial^{4} u}{\partial x^{4}}=\frac{1}{m_{B}+m_{g}} f(x, t)
$$

Using the method of separation of variables, the displacement y can be written as

$$
u(x, t)=\sum_{j=1}^{\infty} \phi_{j}(x) q_{j}(t)
$$

where $\phi_{j}(x)$ are the normalized mode shapes determined above. Substituting this into Equation (94), we obtain

$$
\sum_{j=1}^{\infty} \phi_{j}(x) \frac{d^{2} q_{j}}{d t^{2}}+a^{2} \sum_{j=1}^{\infty} \phi_{j}(x) \frac{d q_{j}}{d t}+d^{2} \sum_{j=1}^{\infty} \frac{d^{4} \phi_{j}(x)}{d x^{4}} q_{j}(t)=\frac{1}{m_{B}+m_{g}} f(x, t)
$$

Multiplying Equation (96) both sides by $\phi_{\mathrm{k}}(\mathrm{x})$ and integrating from 0 to $\mathrm{L}$, we obtain 


$$
\begin{aligned}
& \sum_{j=1}^{\infty} \frac{d^{2} q_{j}}{d t^{2}} \int_{0}^{L} \phi_{j} \phi_{k}+a^{2} \sum_{j=1}^{\infty} \frac{d q_{j}}{d t} \int_{0}^{L} \phi_{j} \phi_{k}+d^{2} \sum_{j=1}^{\infty} q_{j}(t) \int_{0}^{L} \phi_{k} \frac{d^{4} \phi_{j}(x)}{d x^{4}} \\
& =\frac{1}{m_{B}+m_{g}} \int_{0}^{L} \phi_{k} f(x, t)
\end{aligned}
$$

All items in Equation (97) with $\mathrm{j} \neq \mathrm{k}$ on left hand side are zero and $\int_{0}^{L} \phi_{j} \phi_{k}=1$ with $\mathrm{j}=\mathrm{k}$.

The equation can then be rearranged as:

$$
\frac{d^{2} q_{j}}{d t^{2}}+a^{2} \frac{d q_{j}}{d t}+\omega_{n j}{ }^{2} q_{j}=\frac{1}{m_{B}+m_{g}} \int_{0}^{L} \phi_{j}(x) f(x, t)
$$

where $\mathrm{k}$ has been replaced with $\mathrm{j}$ after simplification. Note that for a given value of $\mathrm{j}$, Equation (98) represents force vibration of a single degree-of -freedom spring-massdamping system. Since $\mathrm{j}$ varies from 1 to $\infty$, a cantilever beam can be thought of as a system of an infinite number of single degree-of-freedom spring-mass-damping systems. Consequently, the response of a vibrating cantilever beam may have an infinite number of individual vibration terms.

To get a better understanding of the system and the response of the system, assume that the beam is initially in rest position and subjected to a point force $F(t)$ acting at a point $x_{0}$. In this case, the initial conditions and the force $f(x, t)$ can be written as

$$
\begin{gathered}
u(x, 0)=\frac{\partial u(x, 0)}{\partial t}=0 \\
f(x, t)=\delta\left(x-x_{0}\right) F(t)
\end{gathered}
$$


where $\delta\left(x-x_{0}\right)$ is the Dirac delta function, which Dirac delta function can be characterized by two properties:

1. $\delta\left(x-x_{0}\right)=\left\{\begin{array}{ll}\infty, & x=x_{0} \\ 0, & x \neq x_{0}\end{array}\right.$ and 2. $\int_{0}^{L} \delta\left(x-x_{0}\right) d(x)=d\left(x_{0}\right)$

Since, Equation (99) must be satisfied at all points of the beam, using Equation (55) leads to:

$$
q_{j}(0)=\frac{d q_{j}(0)}{d t}=0
$$

Substituting Equation (100) into Equation (98), the right hand side of equation yields:

$$
\begin{aligned}
& \frac{1}{m_{B}+m_{g}} \int_{0}^{l} \phi_{j}(x) f(x, t) d x=\frac{1}{m_{B}+m_{g}} \int_{0}^{L} \phi_{j}(x) \delta\left(x-x_{0}\right) F(t) d x \\
& =\frac{F(t)}{m_{B}+m_{g}} \int_{0}^{L} \phi_{j}(x) \delta\left(x-x_{0}\right) d x=\frac{F(t)}{m_{B}+m_{g}} \phi_{j}\left(x_{0}\right)
\end{aligned}
$$

This result is due to the sifting property of the Dirac Delta function since $\delta\left(x-x_{0}\right)$ has the effect of sifting the value $\phi_{j}\left(x_{0}\right)$ out of the integral of $\phi_{j}$ on [0, L]. [55] Equation (98) can now be expressed as:

$$
\frac{d^{2} q_{j}}{d t^{2}}+a^{2} \frac{d q_{j}}{d t}+\omega_{n j}^{2} q_{j}=\frac{1}{m_{B}+m_{g}} \phi_{j}\left(x_{0}\right) F(t)
$$

In this dissertation, the electrostatic force applies at the end $\left(\mathrm{x}_{0}=\mathrm{L}\right)$ and the part of harmonic the force, $F(t)=F_{0} \sin (\omega t)$ is considered first, and Equation (102) reduces to:

$$
\frac{d^{2} q_{j}}{d t^{2}}+a^{2} \frac{d q_{j}}{d t}+\omega_{n j}^{2} q_{j}(t)=\frac{1}{m_{B}+m_{g}} \phi_{j}(L) F_{0} \sin (\omega t)
$$


where $F_{0}$ is the peak amplitude of the force. In an underdamped system (the only type considered in this dissertation), the solution of this equation is then expressed by the particular solution:

$$
\begin{aligned}
& q_{p \cdot j}=K_{j} \cos (\omega t)+M_{j} \sin (\omega t) \\
& \frac{d q_{p \cdot j}}{d t}=\omega\left(-K_{j} \sin (\omega t)+M_{j} \cos (\omega t)\right. \\
& \frac{d^{2} q_{p \cdot j}}{d t^{2}}=\omega^{2}\left(-K_{j} \cos (\omega t)-M_{j} \sin (\omega t)\right.
\end{aligned}
$$

where $K_{j}$ and $M_{j}$ are to be determined. Substitute Equation (104) into Equation (102), and collect the cosine and sine terms. Since there is no cosine term in $F(t)$, the cosine terms must equal zero. Similarly, the sine terms must equal the forcing function on the right. This becomes:

$$
\begin{aligned}
& -\omega^{2} M_{j}-a^{2} \omega K_{j}+\omega_{n j}{ }^{2} M_{j}=\frac{F_{0}}{m_{B}+m_{g}} \phi_{j}(L) \\
& -\omega^{2} K_{j}+a^{2} \omega M_{j}+\omega_{n j}{ }^{2} K_{j}=0
\end{aligned}
$$

The solutions of $\mathrm{M}_{\mathrm{J}}$ and $\mathrm{K}_{\mathrm{j}}$ are

$$
\begin{aligned}
& M_{j}=\frac{F_{0}}{\left(m_{B}+m_{g}\right)} \frac{\left(\omega_{n j}^{2}-\omega^{2}\right)}{\left(\omega_{n j}^{2}-\omega^{2}\right)^{2}+\left(a^{2} \omega\right)^{2}} \phi_{j}(L) \\
& K_{j}=\frac{F_{0}}{\left(m_{B}+m_{g}\right)} \frac{-a^{2} \omega}{\left(\omega_{n j}^{2}-\omega^{2}\right)^{2}+\left(a^{2} \omega\right)^{2}} \phi_{j}(L)
\end{aligned}
$$

Equation (104) can also be written in the form of

$$
q_{p \cdot j}(t)=C_{j}^{*} \sin \left(\omega t-\eta_{j}\right)
$$


where the amplitude $C_{j}{ }_{j}^{*}$ and the angle $\eta_{\mathrm{j}}$ are given by

$$
\begin{aligned}
& C_{j}^{*}=\sqrt{M_{j}{ }^{2}+K_{j}{ }^{2}}=\frac{F_{0}}{\left(m_{B}+m_{g}\right)} \frac{1}{\sqrt{\left(\omega_{n \cdot j}{ }^{2}-\omega^{2}\right)^{2}+\left(a^{2} \omega\right)^{2}}} \phi_{j}(L) \\
& \tan \eta_{j}=\frac{M_{j}}{K_{j}}=\frac{\omega_{n \cdot j}{ }^{2}-\omega^{2}}{a^{2} \omega}
\end{aligned}
$$

It is now possible to write $q_{p,}$ in the form

$$
\begin{aligned}
& q_{p \cdot j}(t)=K_{j} \cos (\omega t)+M_{j} \sin (\omega t)= \\
& \frac{F_{0}}{\left(m_{B}+m_{g}\right)} \frac{a^{2} \omega}{\left(\omega_{n \cdot j}{ }^{2}-\omega^{2}\right)^{2}+\left(a^{2} \omega\right)^{2}} \phi_{j}\left(x_{0}\right) \cos (\omega t)+ \\
& \frac{F_{0}}{\left(m_{B}+m_{g}\right)} \frac{\left(\omega^{2}-\omega_{j}^{2}\right)}{\left(\omega_{n \cdot j}{ }^{2}-\omega^{2}\right)^{2}+\left(a^{2} \omega\right)^{2}} \phi_{j}\left(x_{0}\right) \sin (\omega t)
\end{aligned}
$$

Now consider the solution to the homogeneous version of Equation (103), namely that with the right hand side equal to 0

$$
\frac{d^{2} q_{j}}{d t^{2}}+a^{2} \frac{d q_{j}}{d t}+\omega_{n j}^{2} q_{j}(t)=0
$$

This has the homogeneous solution $q_{h \cdot j}$; for underdamped system this becomes:

$$
q_{h \cdot j}=e^{-\zeta \omega_{n j} t}\left(c_{5} \cos \omega_{d} t+c_{6} \sin \omega_{d} t\right)
$$

where $\omega_{d, j}=\frac{1}{2} \sqrt{4 \omega_{n \cdot j}{ }^{2}-a^{4}}=\omega_{n \cdot j} \sqrt{1-\left(\frac{a^{2}}{2 \omega_{n, j}}\right)^{2}}=\omega_{n \cdot j} \sqrt{1-\varsigma^{2}}$ and $\varsigma=\frac{a^{2}}{2 \omega_{n j}}$ is the damping ratio. The restriction of underdamped response means that the damping ratio $\varepsilon$ is 
less than the critical value of 1 . Since $q_{j}(t)=q_{h \cdot j}(t)+q_{p \cdot j}(t)$ is also a solution to Equation (94), the general solution is obtained as:

$q_{j}(t)=q_{h}(t)+q_{p}(t)=e^{-\zeta \omega_{n j} t}\left(c_{5} \cos \omega_{d} t+c_{6} \sin \omega_{d} t\right)+K_{j} \cos (\omega t)+M_{j} \sin (\omega t)$

The $c_{5}$ and $c_{6}$ are determined from the initial conditions. The second condition gives

$$
\begin{aligned}
& q_{j}(0)=c_{5}-C_{j}^{*}=0 \rightarrow c_{5}=-K_{j} \\
& \frac{d q_{j}(0)}{d t}=-\xi \omega_{n} c_{5}+c_{6} \omega_{d}+\omega M_{j}=0 \rightarrow c_{6}=\frac{-\xi \omega_{n} K_{j}+\omega M_{j}}{\omega_{d}}
\end{aligned}
$$

From Equation (112) the general response of the underdamped $\mathrm{j}^{\text {th }}$ mode becomes:

$$
q_{j}(t)=e^{-\zeta \omega_{n j} t}\left(-K_{j} \cos \omega_{d} t+\frac{-\xi \omega_{n} K_{j}+\omega M_{j}}{\omega_{d}} \sin \omega_{d} t\right)+K_{j} \cos (\omega t)+M_{j} \sin (\omega t)
$$

Substituting Equation (100) into Equation (82), the beam displacement is obtained as:

$$
\begin{aligned}
& u(x, t)=\sum_{j} \varphi_{j}(x) q_{j}(t)= \\
& \sum_{j=0}^{\infty} \phi_{j}(x)\left[e^{-\xi \omega_{n j} t}\left(-K_{j} \cos \omega_{d} t+\frac{-\xi \omega_{n} K_{j}+\omega M_{j}}{\omega_{d}} \sin \omega_{d} t\right)+K_{j} \cos (\omega t)+M_{j} \sin (\omega t)\right] \\
& \text { where } M_{j}=\frac{F_{0}}{\left(m_{B}+m_{g}\right)} \frac{\left(\omega_{n j}{ }^{2}-\omega^{2}\right)}{\left(\omega_{n j}{ }^{2}-\omega^{2}\right)^{2}+\left(a^{2} \omega\right)^{2}} \phi_{j}(L) \\
& K_{j}=\frac{F_{0}}{\left(m_{B}+m_{g}\right)} \frac{-a^{2} \omega}{\left(\omega_{n j}{ }^{2}-\omega^{2}\right)^{2}+\left(a^{2} \omega\right)^{2}} \phi_{j}(L)
\end{aligned}
$$

An example of $u(x, t)$ is plotted using Matlab in Figure 89 for a particular solution. The lateral vibration of cantilever beam is demonstrated in 3D which includes 
changes of displacement $\mathrm{u}(\mathrm{m})$, time $(\mathrm{s})$, and beam position $\mathrm{x}(\mathrm{m})$. The driving frequency in this case is set as $86 \mathrm{kHz}$ which is closed to the natural frequency $\left(\omega_{\mathrm{n}}\right)$. The vibration starts a beat signal in first several cycles and then goes harmonic vibration.
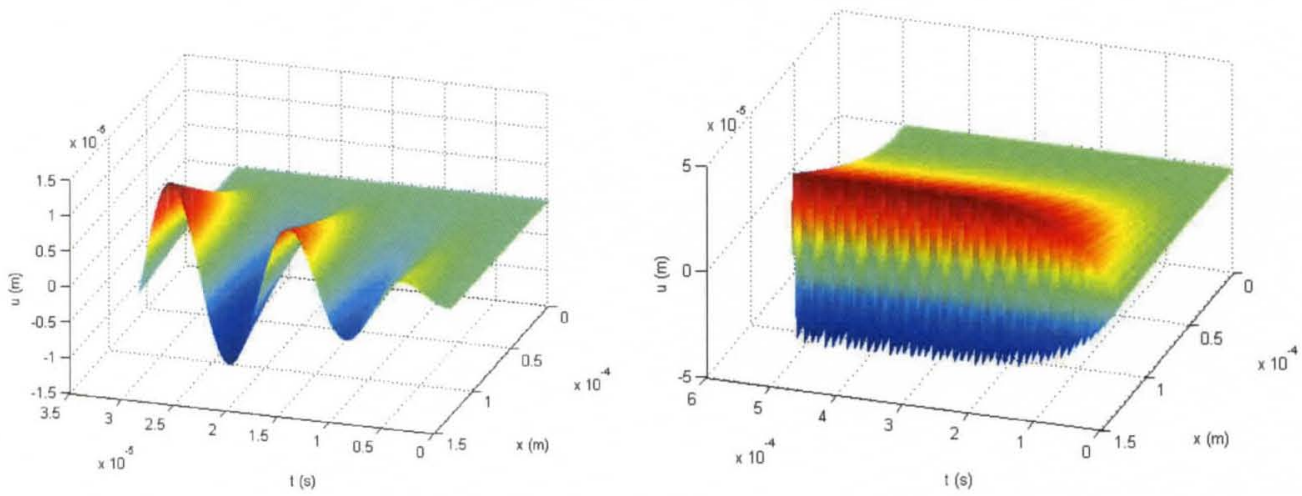

Figure 89. Vertical displacement of cantilever beam changes with time. (Left) 2 cycles;

(Right) 40 cycles

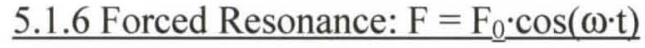

When force equals $F_{0} \cdot \cos (\omega \cdot t)$, the derivation of beam behavior is similar for sinusoidal force as discussed in Section 5.1.3. However, Equation (106) in this case becomes as:

$$
\begin{aligned}
& -\omega^{2} M_{j}-a^{2} \omega K_{j}+\omega_{n j}{ }^{2} M_{j}=0 \\
& -\omega^{2} K_{j}+a^{2} \omega M_{j}+\omega_{n j}{ }^{2} K_{j}=\frac{F_{0}}{m_{B}+m_{g}} \phi_{j}(L)
\end{aligned}
$$

The solutions of $\mathrm{M}_{\mathrm{j}}$ and $\mathrm{K}_{\mathrm{j}}$ are as: 


$$
\begin{aligned}
& M_{3 j}=\frac{F_{0}}{\left(m_{B}+m_{g 3}\right)} \frac{a_{3}{ }^{2} \cdot \omega}{\left(\omega_{n j 3}{ }^{2}-\omega^{2}\right)^{2}+\left(a_{3}{ }^{2} \cdot \omega\right)^{2}} \phi_{j}(L) \\
& K_{3 j}=\frac{F_{0}}{\left(m_{B}+m_{g 3}\right)} \frac{\left(\omega_{n j 3}{ }^{2}-\omega^{2}\right)}{\left(\omega_{n j 3}{ }^{2}-\omega^{2}\right)^{2}+\left(a_{3}{ }^{2} \cdot \omega\right)^{2}} \phi_{j}(L)
\end{aligned}
$$

The form of expression of $u(x, t)$ corresponding to $F=F_{0} \cdot \cos ((\omega \cdot t)$ is similar with $u(x, t)$ corresponding to $F=F_{0} \cdot \sin (\omega \cdot t)$ in Equation (115). Except the change value of $M_{j}$ and $K_{j}$ which are shown in Equation (117).

\subsubsection{Steady State Solution of Forced Resonance}

The general solution of the nonhomogeneous equation is $q=q_{h}+q_{p}$; this called the transient solution. The solution of homogeneous equation $\left(q_{h}\right)$ approaches zero as $\mathrm{t}$ goes to infinity if any damping is present $(\xi>0)$. Practically, it is zero after a sufficiently long time. Therefore, as $\mathrm{t} \rightarrow \infty, \mathrm{q} \rightarrow q_{p}$. Hence, after a sufficiently long time, the output will become steady state, corresponding to a purely sinusoidal input leading to a harmonic oscillation whose frequency is identical to that of the input. This is what happens in practice, because no physical system is completely undamped. [55]

Resonance of the microcantilever beam which can be observed visually and experimentally is due to long-term forced resonance. In this case, the homogeneous solution part in Equation (115) could be cleared out. The long-term solution for $F=$ $\mathrm{F}_{0} \cdot \sin (\omega \cdot \mathrm{t})$ is as:

$$
\left.u_{2}=\sum_{j} \varphi_{j}(x) q_{p}(t)=\sum_{j=0}^{\infty} \phi_{j}(x)\left[K_{2 j} \cos \omega t+M_{2 j} \sin \omega t\right)\right]
$$


The long-term solution of beam for $F=F_{0} \cdot \cos (\omega \cdot t)$ is as:

$$
\left.u_{3}=\sum_{j} \varphi_{j}(x) q_{p}(t)=\sum_{j=0}^{\infty} \phi_{j}(x)\left[K_{3 j} \cos \omega t+M_{3 j} \sin \omega t\right)\right]
$$

The peak magnitude of beam tip displacement for $u_{2}$ and $u_{3}$ are same which could be expressed as:

$$
U=\sqrt{\left(\sum_{j=0}^{\infty} \phi_{j}(L)^{*} K_{j}\right)^{2}+\left(\sum_{j=0}^{\infty} \phi_{j}(L)^{*} M_{j}\right)^{2}}
$$

The steady-state amplitude for cantilever beam is $U_{1}=\frac{F_{1} \cdot L^{3}}{3 E I}$. This will correspond to $\omega \rightarrow 0$. The system $U$ goes to $\infty$ when $\omega=\omega_{\mathrm{n}}$. Figure 90 shows the curve of $U$ vs. frequencies for $F=F_{0} \cdot \sin (\omega \cdot t)$. The cantilever beam resonates in air at room temperature in $1 \mathrm{~atm}$. The frequency is swept from $80 \mathrm{kHz}$ to $100 \mathrm{kHz}$. The frequency corresponding to the peak of the curve is the resonance frequency of cantilever beam. 


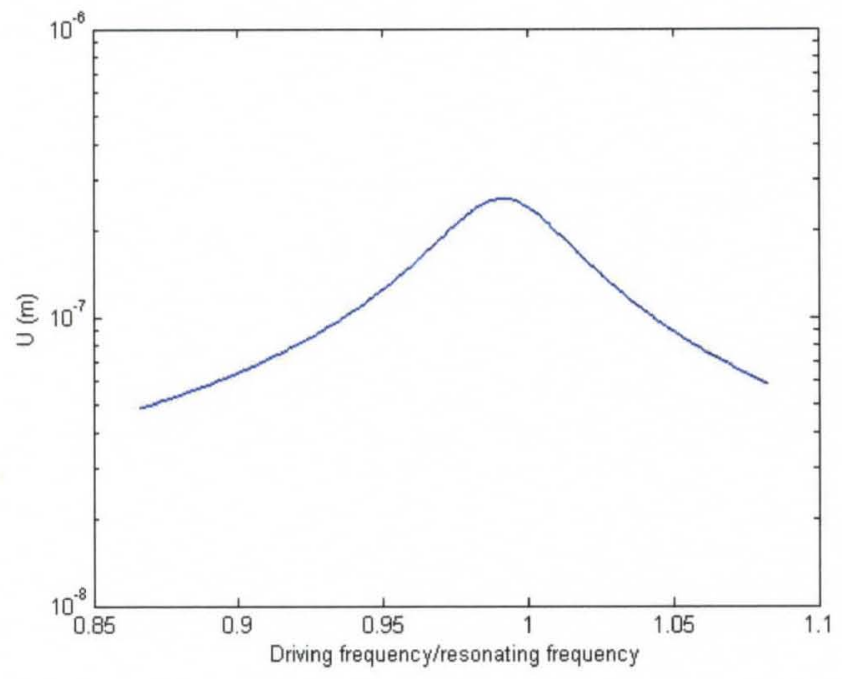

Figure 90. Response of a vibration cantilever beam versus frequencies: $F=F_{0} \cdot \sin (\omega \cdot t)$.

As discussed in Section 4.3, the electrostatic force including a sinusoidal force and also a cosine force. The cosine force has frequency of $2 \omega$. The $U$ versus frequency is plotted in Figure 91. Due to microcantilever beam being driven by $2 \omega$, it resonates at frequency of $\omega$ which $1 / 2$ of the driving frequency.

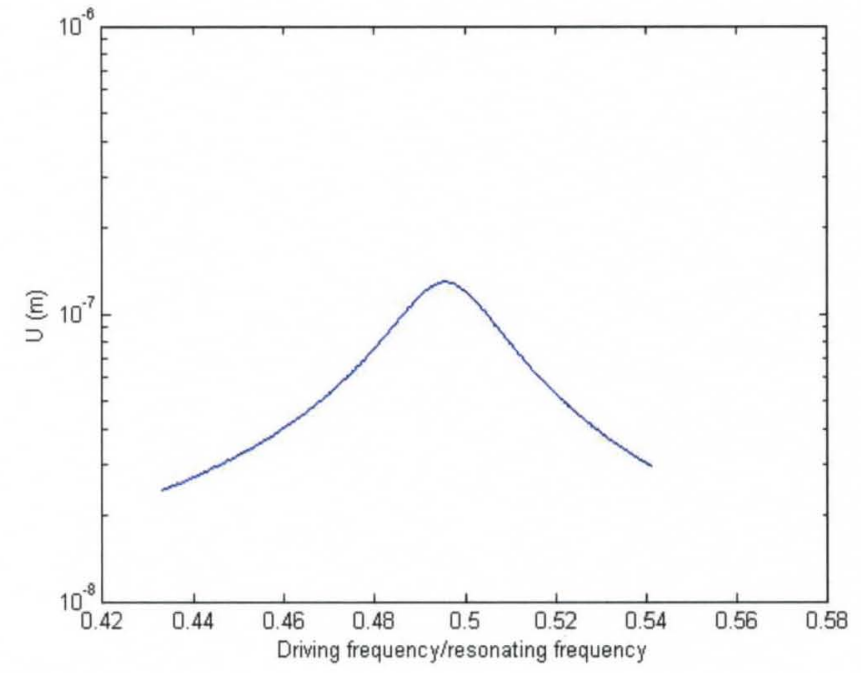

Figure 91. Response of a vibration cantilever beam versus frequencies: $F=F_{0} \cdot \cos (\omega \cdot t)$. 


\subsection{Device Behavior Under Driving Electrode Actuation}

In this section, the long-term displacement of microcantilever beam responding to driving electrostatic force is considered. The electrostatic force as shown is:

$$
F=k h_{0}+k h_{1} \sin (\omega t)-k h_{2} \cos (2 \omega t)
$$

Then the displacement of microcantilever beam responding to the electrostatic force shown above is expressed as:

$$
u=u_{1}+u_{2}+u_{3}
$$

Substituting $u 2$ and $u 3$ into Equation (118) and (119) to get long term solution, then the expression becomes:

$$
\begin{aligned}
& u=c_{00}+c_{10} \sin (\omega t)+c_{11} \cos (\omega t)+c 20 \sin (2 \omega t)+c 21 \cos (2 \omega t) \\
& \left.\left.\frac{F_{1} L^{3}}{3 E I}+\sum_{j=0}^{\infty} \phi_{j}(x)\left[K_{2 j} \cos \omega t+M_{2 j} \sin \omega t\right)\right]+\sum_{j=0}^{\infty} \phi_{j}(x)\left[K_{3 j} \cos \omega t+M_{3 j} \sin \omega t\right)\right]
\end{aligned}
$$

where

$$
\begin{aligned}
& c_{00}=\frac{F_{1} L^{3}}{3 E I}, \quad c_{10}=\sum_{j=0}^{\infty} \phi_{j}(x) M_{2 j}, \quad c_{11}=\sum_{j=0}^{\infty} \phi_{j}(x) K_{2 j} \\
& c_{20}=\sum_{j=0}^{\infty} \phi_{j}(x) M_{3 j}, \quad c_{21}=\sum_{j=0}^{\infty} \phi_{j}(x) K_{3 j}
\end{aligned}
$$

where 


$$
\begin{aligned}
& F_{1}=k h_{0}, F_{2}=k h_{1}, F_{3}=k h_{2} \\
& k=K\left(\frac{E h}{4}\right)\left(\frac{b}{L}\right)^{3}, K=\frac{\delta_{0}}{\left(\Delta V_{0}\right)^{2}} \\
& h_{0}=\left(V_{0}-V_{B}\right)^{2}+\frac{1}{2}\left(V_{E}\right)^{2}, h_{1}=2 V_{E}\left(V_{0}-V_{B}\right), h_{2}=\left(\frac{1}{2}\right)\left(V_{E}\right)^{2} \\
& V_{0}=0 \mathrm{~V}, V_{B}=5 \mathrm{~V}, V_{E}=10 \mathrm{~V} \\
& \delta_{0}=0.012 \mu \mathrm{m}, \Delta V_{0}=10 \mathrm{~V}
\end{aligned}
$$

Once the factors of Equation (124) are determined, the resonance behavior of microcantilever beam could be achieved. By using equations above, the displacement factors calculated for microcantilever beam driven by series different frequencies. Those factor values corresponding to different driven frequency individually listed in Table 23. The static row in the table corresponds to beam response with no inertial effects; in this case, the displacement factors are not available for this case. Driven frequencies of $1 \mathrm{kHz}$ (low frequency well below $\omega_{n}$ ), $\omega_{n} / 3, \omega_{n} / 2$, and $\omega_{n}$ are chosen for demonstration below

\begin{tabular}{|c|c|c|c|c|c|}
\hline & $c_{00}$ & $c_{10}$ & $c_{11}$ & $c_{20}$ & $c_{21}$ \\
\hline Static & - & - & - & - & - \\
\hline $1 \mathrm{kHz}$ & $9.0000 \mathrm{E}-09$ & $-1.1995 \mathrm{E}-08$ & $2.9458 \mathrm{E}-12$ & $-3.1953 \mathrm{E}-12$ & $-5.9997 \mathrm{E}-09$ \\
\hline$\omega_{n} / 3$ & $9.0000 \mathrm{E}-09$ & $-1.3502 \mathrm{E}-08$ & $1.8502 \mathrm{E}-10$ & $-5.4575 \mathrm{E}-10$ & $-1.0809 \mathrm{E}-08$ \\
\hline$\omega_{n}$ & $9.0000 \mathrm{E}-09$ & $8.0521 \mathrm{E}-08$ & $2.2763 \mathrm{E}-07$ & $-6.6638 \mathrm{E}-11$ & $1.7232 \mathrm{E}-09$ \\
\hline$\omega_{n} / 2$ & $9.0000 \mathrm{E}-09$ & $-1.5999 \mathrm{E}-08$ & $4.2032 \mathrm{E}-10$ & $-1.1382 \mathrm{E}-07$ & $4.0260 \mathrm{E}-08$ \\
\hline
\end{tabular}

Table 23. Displacement factors (in $\mathrm{m}$ ) versus different frequency.

For $F(t)=k \Delta V^{2}, \Delta V^{2}=\left(V_{e}-V_{B}\right)^{2}, V_{e}=V_{E} \sin (\omega t)$ is the driving sinusoidal signal . Figure 92, Figure 93 and Figure 94 shown the $\Delta \mathrm{V}^{2}$ versus time corresponding to $V_{B}=0, V_{B}=5$ and $V_{B}=10$ separately. The setting of $V_{B}=5$ leads most complex shape of $\Delta \mathrm{V}^{2}$ changing with time and is chosen to be the setting for following plots. 


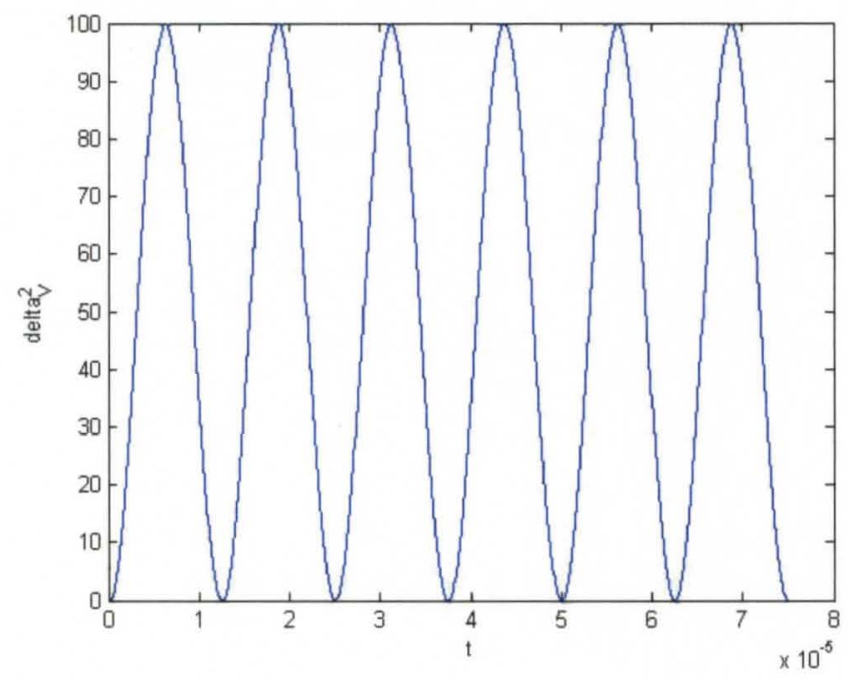

Figure 92. Square of $\Delta \mathrm{V}=\left(\mathrm{V}_{\mathrm{e}}-\mathrm{V}_{\mathrm{B}}\right)^{2}$ for $V_{E}=10, V_{B}=0$.

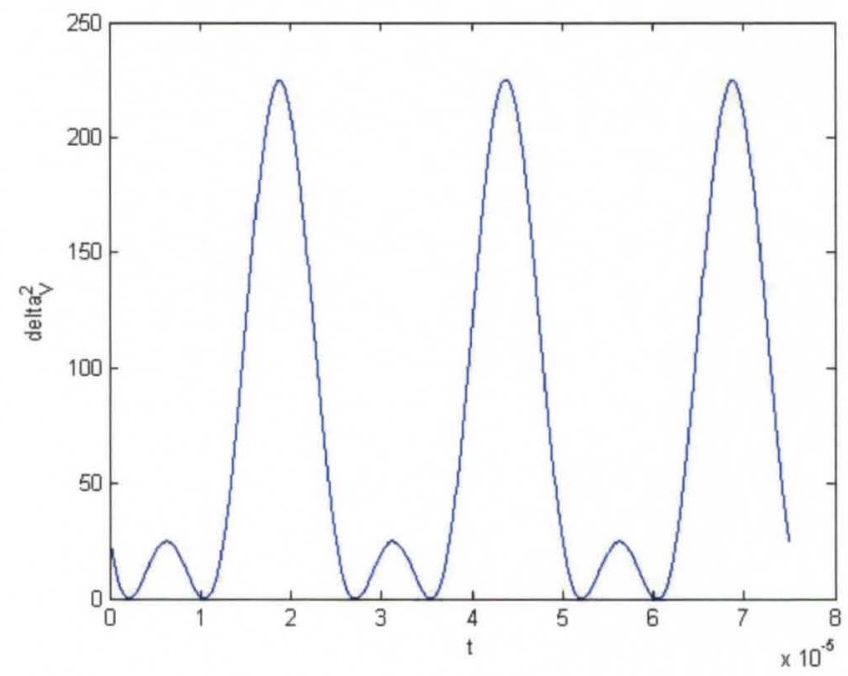

Figure 93. Square of $\Delta \mathrm{V}=\left(\mathrm{V}_{\mathrm{e}}-\mathrm{V}_{\mathrm{B}}\right)^{2}$ for $V_{E}=10, V_{B}=5$. 


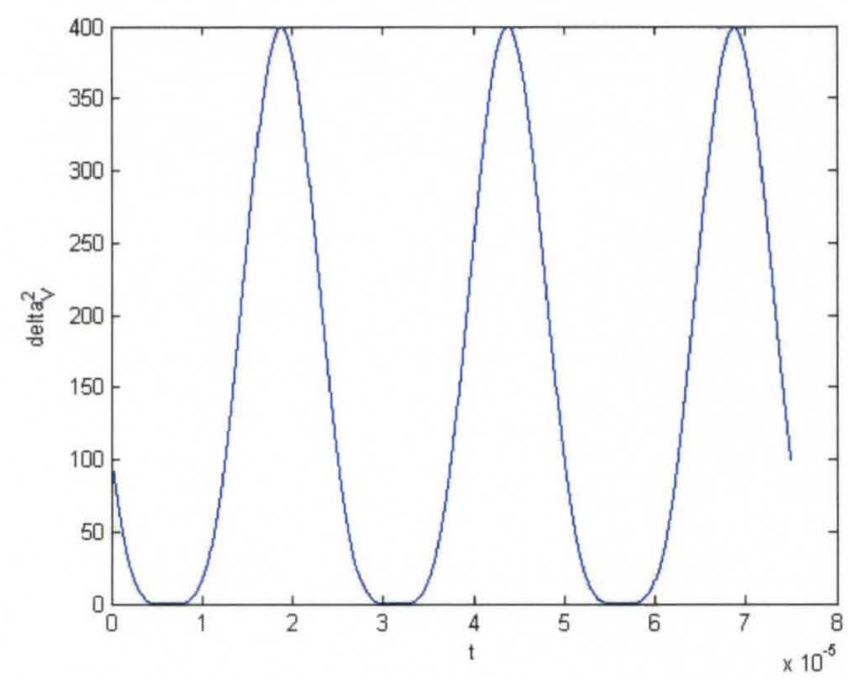

Figure 94. Square of $\Delta \mathrm{V}=\left(\mathrm{V}_{\mathrm{e}}-\mathrm{V}_{\mathrm{B}}\right)^{2}$ for $V_{E}=10, V_{B}=10$.

The Figure 95,Figure 96,Figure 97,Figure 98 and Figure 99 shows the plots of displacement of microcantilever beam versus time for the case of $V_{E}=10, V_{B}=5$ when the beam is in air at $1 \mathrm{~atm}$. When driven frequency is very low relative to the first natural frequency $\left(\omega_{n 1}=92.768 \mathrm{kHz}\right)$, the displacement of beam tip over time (Figure 95) is nearly identical to the static displacement under the identical $F(t)$, which is simply a scaled version of $\Delta \mathrm{V}^{2}$ (Figure 96). Cook [56] mentions that for a cyclic forcing function that if the frequency is less than one-third the first natural frequency of the structure, it will produce an undamped maximum response only about $10 \%$ greater than the static response to the amplitude of the load. Figure 97 shows that corresponding displacement of beam which driven by $\omega_{n} / 3$ has the same curve shape of response of static force which is shown in Figure 95. The approximate displacement's peak value from above plot shows that $\omega_{n} / 3$ leads about $20 \%$ greater maximum response than the static case. As expected, when the driving electrode frequency equals $\omega_{n}$, a much larger displacement 
occurs than in the static case (Figure 98). Of particular interest to the devices in this study, however, is the displacement for the case when the driven frequency is $\omega_{n} / 2$ (Figure 99); in this case, large displacements at a frequency equal to twice times of the driving frequency occur due to the portion of the driving force that is proportional to cos $2 \omega t$.

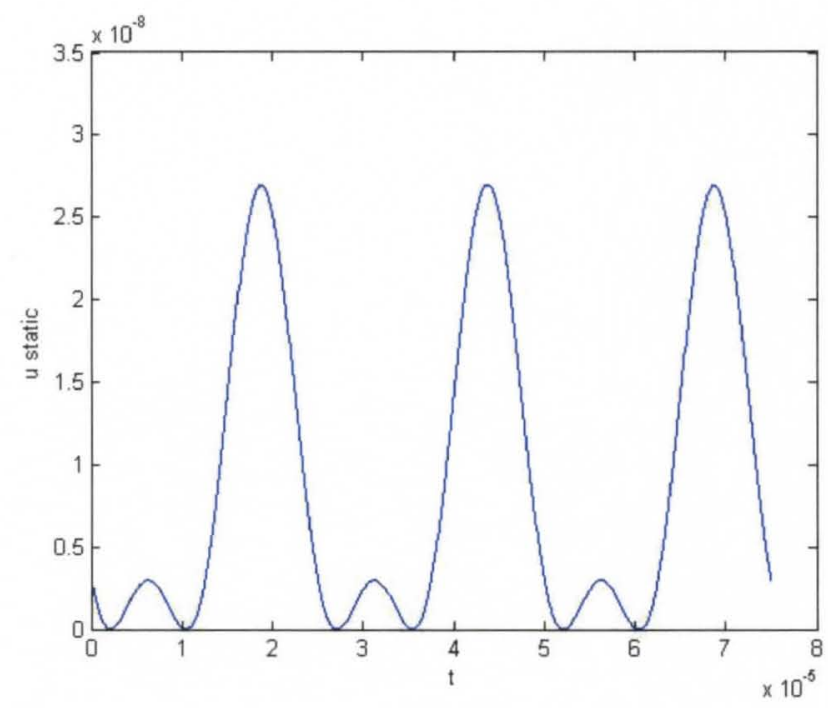

Figure 95. Beam displacement for $V_{E}=10, V_{B}=5$ with driving electrode voltage frequency of $1 \mathrm{kHz}$. 


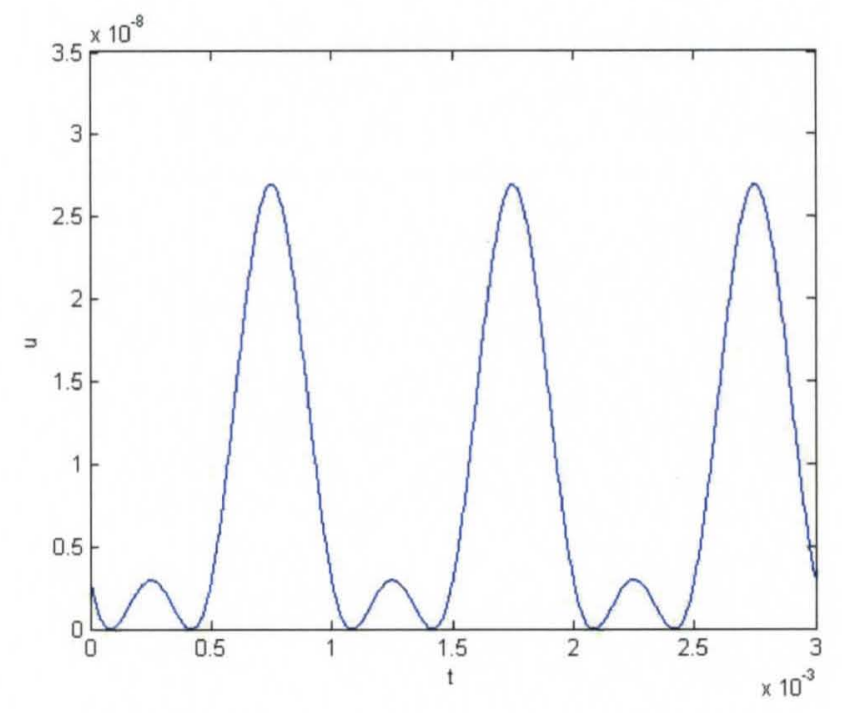

Figure 96. Beam displacement for static force for $V_{E}=10, V_{B}=5$.

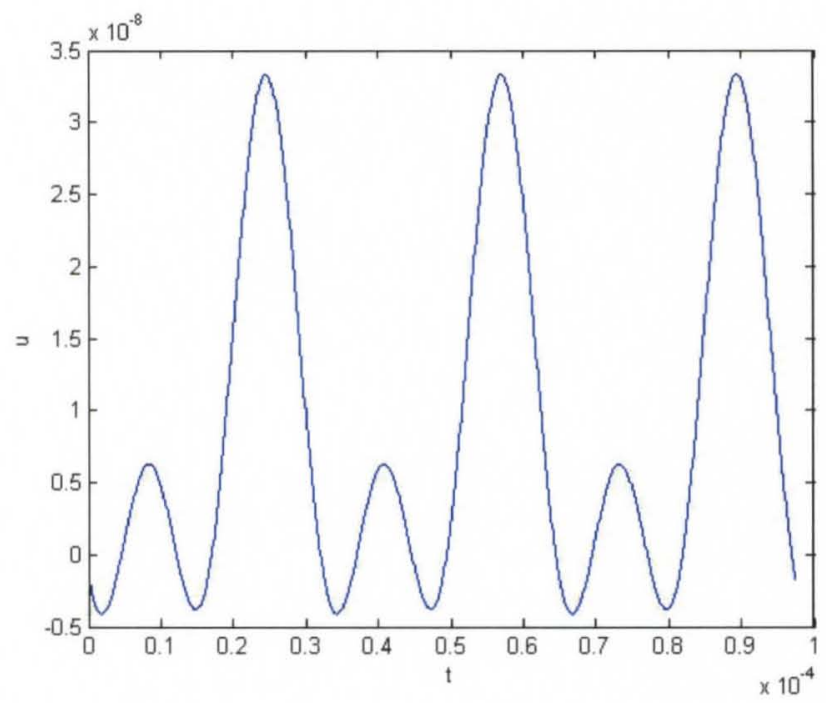

Figure 97. Beam displacement for $V_{E}=10, V_{B}=5$ with driving electrode voltage frequency of $\omega_{n} / 3$. 


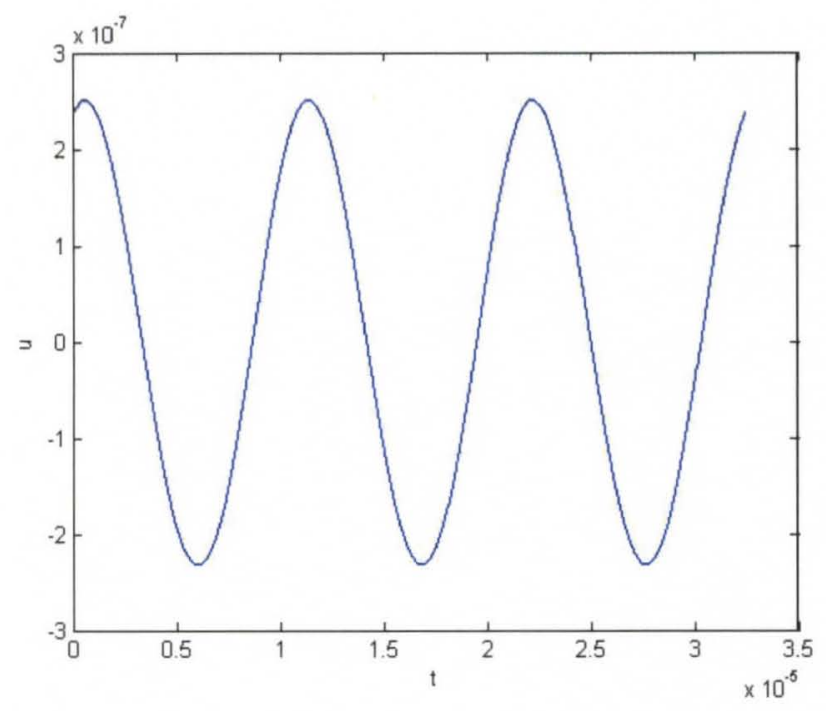

Figure 98. Beam displacement for $V_{E}=10, V_{B}=5$ with driving electrode voltage frequency of $\omega_{\mathrm{n}}$

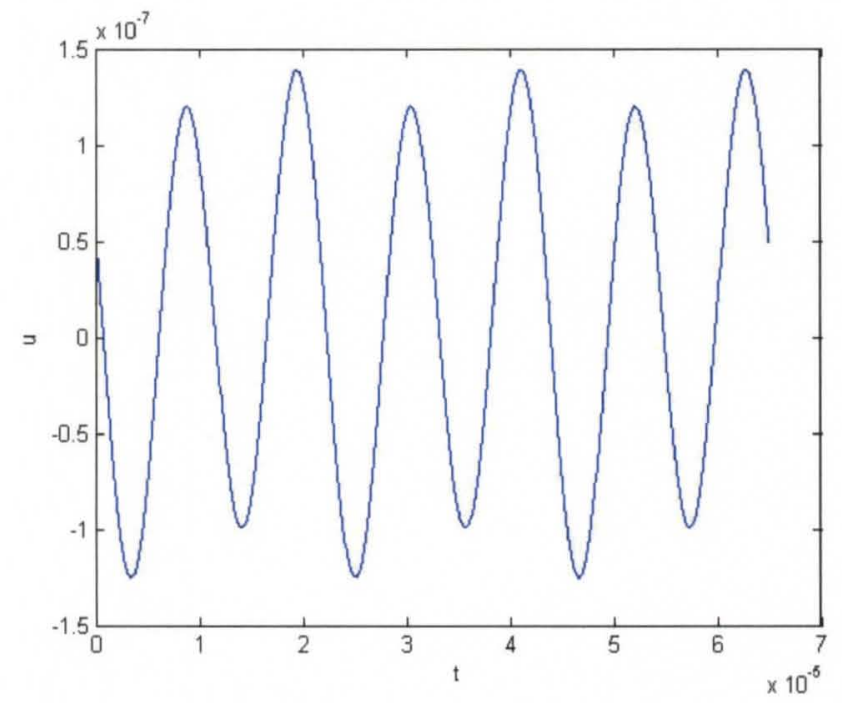

Figure 99. Beam displacement for $V_{E}=10, V_{B}=5$ with driving electrode voltage frequency of $\omega_{n} / 2$.

The peak displacement of tip beam, $\mathrm{u}$, versus swept frequency is shown in Figure 100 where the frequency is swept from 40 to $50 \mathrm{kHz}, 80$ to $100 \mathrm{kHz}$, and 40 to $100 \mathrm{kHz}$. 
This result shows the clear resonant peaks in the regions near a driving electrode frequency of both $\omega_{n}$ and $\omega_{n} / 2$. This is significant because it demonstrates a clear mechanism by which beams which have a theoretical first natural frequency $\omega_{n 1}$ in the 80 $-100 \mathrm{kHz}$ range can be made to resonate using a driving electrode voltage in the 40-50 $\mathrm{kHz}$ range (as occurs with beams studied in this dissertation and in previous related work at the University of Louisville). 

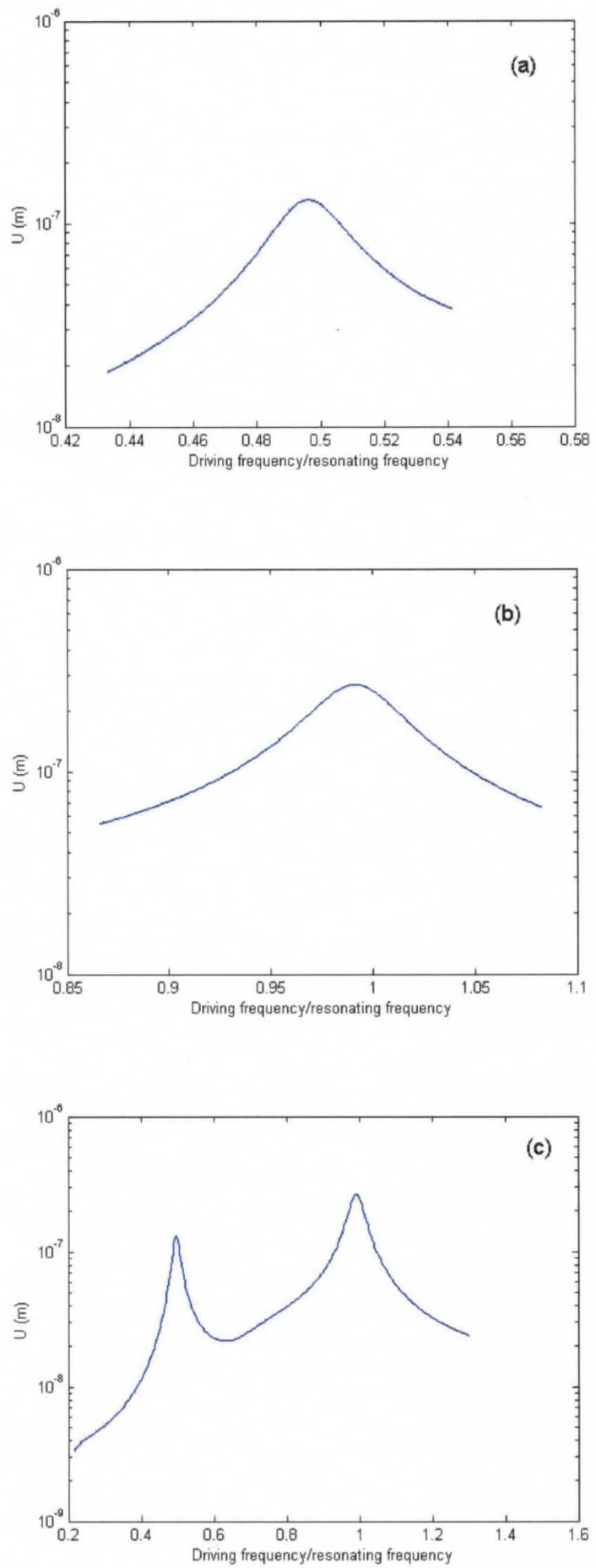

Figure 100. Peak of displacement versus frequency for $u$ : frequency swept (a) from 40-50 kHz; (b) from 80-100 kHz; (c) from 20-120 kHz. 


\subsection{Damping Effect Characterization}

The undamped system driven by a sinusoidal force will tend to $U / U_{0} \rightarrow \infty$ as $\omega \rightarrow \omega_{n}$. However, the presence of damping leads to a finite peak value $U / U_{0}$ at a frequency $\omega<\omega_{n}$ for frequencies in the region around $\omega_{n}$. For validation purposes, the displacement solution at any frequency can also be compared to the static solution which is identical to the case $\omega \rightarrow 0$; for the cantilever beam with an end shear load this becomes $U_{0}=\left(\frac{L^{3}}{3 E I}\right) F_{0}$

\section{$\underline{\text { 5.3.1 Viscosity - Ideal Gas }}$}

An ideal gas has a dynamic viscosity $\mu$ that is constant versus pressure; this was demonstrated by Maxwell using the kinetic theory of gas molecules [57]. In this case, the damping load acting on the beam due to the viscous effect of a surrounding gas from Equation (123) can be used to find the beam displacement via $\mathrm{M}_{j}$ and $\mathrm{K}_{\mathrm{j}}$, both of which are related with $a^{2}=\frac{c_{g}}{m_{B}+m_{g}}=\frac{Y \cdot m_{c^{\prime}} \cdot k^{\prime} \cdot \omega}{m_{B}+m_{g}}$. Since $m_{c}=\rho b h$ and $\mathrm{k}^{\prime}$ is a function of $\sqrt{\frac{\omega \mu}{\rho}}$, it is clear that $\mathrm{a}^{2}$ is dependent on $\rho$. It is clear that the beam under full vacuum should experience no damping effects (i.e. $\mathrm{a}^{2} \rightarrow 0$ ). This was considered using the analysis based on vibration theory and Stokes oscillating cylinder model with the dynamic viscosity kept constant and the density $\rho=N \rho_{0}$, where $N$ is the number of atmospheres of air surrounding the beam and $\rho_{0}$ is the density of air at 1 atmosphere; in this case, the value $m_{c}$ similarly scales as $m_{c}=N \rho_{0} b h$. Since $Y, m_{B}$ and $w$ are constant 
as the density changes and the value of $\mathrm{mg}$ is small relative to $\mathrm{m}_{\mathrm{B}}$, the undamped case will only occur if the product $\mathrm{m}_{\mathrm{c}} \mathrm{k}^{\prime}$ tends to 0 as $\mathrm{N} \rightarrow 0$. This is not the case, however, as demonstrated and listed in Table 24; these results also show that the damping load $\left(\mathrm{c}_{\mathrm{g}}\right)$ does not become 0 even at infinitesimally low pressures nor does the peak displacement tend towards a large value even though the system is driven at the first natural frequency. This indicates that there is a problem with simply using the damping loads developed using a fluid dynamics approach with gas viscosity that is constant even down to extremely low pressures.

\begin{tabular}{|c|c|c|c|}
\hline $\begin{array}{c}\text { Pressure } \\
(\mathrm{atm})\end{array}$ & $\begin{array}{c}\mathrm{k}^{\prime} \mathrm{m}_{\mathrm{c}} \\
(\mathrm{N} / \mathrm{m})\end{array}$ & $\begin{array}{c}\mathrm{c}_{\mathrm{g}} \\
(\mathrm{N} / \mathrm{s})\end{array}$ & $\begin{array}{c}\delta_{\max } \\
(\mathrm{m})\end{array}$ \\
\hline 1 & $1.77 \mathrm{E}-10$ & $1.34 \mathrm{E}-04$ & $2.37 \mathrm{E}-07$ \\
\hline 0.1 & $1.25 \mathrm{E}-10$ & $1.02 \mathrm{E}-04$ & $3.43 \mathrm{E}-07$ \\
\hline 0.01 & $9.05 \mathrm{E}-11$ & $7.36 \mathrm{E}-05$ & $4.75 \mathrm{E}-07$ \\
\hline 0.0001 & $5.98 \mathrm{E}-11$ & $4.86 \mathrm{E}-05$ & $7.20 \mathrm{E}-07$ \\
\hline $1.00 \mathrm{E}-06$ & $4.45 \mathrm{E}-11$ & $3.62 \mathrm{E}-05$ & $9.67 \mathrm{E}-07$ \\
\hline $1.00 \mathrm{E}-09$ & $3.21 \mathrm{E}-11$ & $2.61 \mathrm{E}-05$ & $1.34 \mathrm{E}-06$ \\
\hline $1.00 \mathrm{E}-12$ & $2.51 \mathrm{E}-11$ & $2.04 \mathrm{E}-05$ & $1.71 \mathrm{E}-06$ \\
\hline $1.00 \mathrm{E}-15$ & $2.06 \mathrm{E}-11$ & $1.68 \mathrm{E}-05$ & $2.07 \mathrm{E}-06$ \\
\hline
\end{tabular}

Table 24. Parameters versus pressure.

\subsubsection{Viscosity - Rarefied Gas and Knudsen Number}

Blom [22] indicated three regimes of beam damping behavior: 1) intrinsic; 2)

molecular (or Knudsen); and 3) viscous. The intrinsic domain corresponds to extremely low pressure in which damping effects are entirely negligible. The viscous region is at a higher pressure where traditional viscosity relationships apply such as those used in the previous section (i.e. dynamic viscosity is independent of pressure). Between these lies the molecular (or Knudsen) region. In this domain, the kinetic theory of gases is used to develop an alternative set of damping loads that differ from those of traditional viscosity. 
For the devices considered by Blom, with beam width of $0.5 \mathrm{~mm}$, the molecular region was defined as pressure $\mathrm{p}=1-100 \mathrm{~Pa}$, with the regions above and below corresponding to viscous and intrinsic regions, respectively.

The change of behavior from the viscous to the Knudsen regime is accompanied by a drop in the effective viscosity of the gas. Recently, Michalis et al [58] performed Monte Carlo simulations of nitrogen molecules traveling in a nanoscale channel at $300^{\circ} \mathrm{K}$; using the resulting wall loads, an effective viscosity was calculated as a function of the Knudsen number, which is defined as:

$$
K_{n}=\frac{\lambda}{L}
$$

where "the mean free path $\lambda$ is the distance a molecule travels, on the average, between collisions" and $\mathrm{L}$ is a characteristic body dimension. For air, $\lambda=2.27 \times 10^{-5} \mathrm{~T} / \mathrm{p}$, where $\mathrm{T}$ is the temperature in Kelvin and $\mathrm{p}$ is the pressure in $\mathrm{Pa}$. [43] After reviewing several theories of the behavior of low-pressure gas behavior, Michalis et al [58] recommend drag effects be calculated using an effective viscosity $\mu_{\mathrm{e}}$ of the form:

$$
\mu_{e}=\mu_{0} \frac{1}{1+a K_{n}}
$$

where $\mu_{0}$ is the traditional dynamic viscosity of the gas (independent of pressure) and $a$ is a parameter that may vary depending on the gas; the value of $a=2$ worked well for the nitrogen simulation and seems consistent with other theories that are compared in the article. This leads to a dramatic drop in viscosity as Knudsen number goes from small 
values (viscous region) to large values (molecular / Knudsen region) as shown in Figure 101.

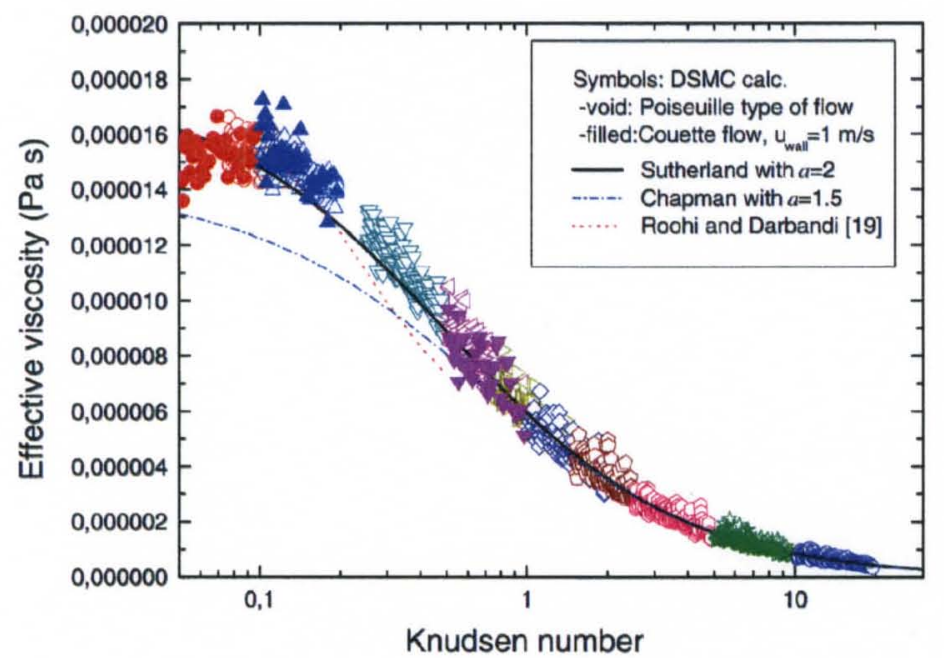

Figure 101. Effective viscosity me for nitrogen at $300^{\circ} \mathrm{K}$ from simulation and compared to several models; the solid line corresponds to Eq. (127) with $a=2$. [58]

The findings described by Blom are consistent with those of the effective viscosity model above. Assuming properties similar to air at $300^{\circ} \mathrm{K}$ using the recommended parameter of $\mathrm{a}=2$ leads to an effective viscosity of:

$$
\begin{aligned}
& p=1 \mathrm{~Pa} \quad \mathrm{~L}=0.500 \mathrm{~mm} \rightarrow K n=13.62 \quad \rightarrow \quad \mu_{e}=0.03541 \mu_{0} \\
& p=100 \mathrm{~Pa} \quad \mathrm{~L}=0.500 \mathrm{~mm} \rightarrow K n=0.1362 \rightarrow \mu_{e}=0.78592 \mu_{0}
\end{aligned}
$$

which entirely consistent with the reported range of $1-100$ Pa representing the molecular (Knudsen) region for the devices considered in Blom.

The devices studied at the University of Louisville are roughly 300 times smaller than those studied by Blom; therefore, the Knudsen regime will be encountered at a much higher pressure. Using a characteristic length $\mathrm{L}$ in the Knudsen number equal to the 
effective diameter of the beam cross-section $(1.674 \mu \mathrm{m})$, the effective viscosity can be estimated as:

$$
K_{n}=\frac{0.03923}{N} \rightarrow \mu_{e}=\mu_{0} \frac{1}{1+0.07846 / N}
$$

where $\mathrm{N}$ is the number of atmospheres of the air surrounding the beam. The effective viscosity drops to $90 \%$ of the pressure-independent value for $\mathrm{N}=0.706$ (537 Torr) and to $10 \%$ of that same value when $\mathrm{N}=0.00872$ (6.6 Torr). If this model is adopted, the values of $\mathrm{k}^{\prime} \cdot \mathrm{m}_{\mathrm{c}}$ and $\mathrm{c}_{\mathrm{g}}$ both decrease sharply as the pressure of the air surrounding the beam tends towards 0 as shown in Table 25.

\begin{tabular}{|c|c|c|}
\hline $\begin{array}{c}\text { Pressure } N \\
(\mathrm{~atm})\end{array}$ & $\begin{array}{c}\mathrm{k}^{\prime} \mathrm{m}_{\mathrm{c}} \\
(\mathrm{N} / \mathrm{m})\end{array}$ & $\mathrm{c}_{\mathrm{g}}$ \\
\hline $1.00 \mathrm{E}-05$ & $4.5963 \mathrm{E}-15$ & $7.4712 \mathrm{E}-09$ \\
\hline $1.00 \mathrm{E}-06$ & $4.5963 \mathrm{E}-16$ & $7.4716 \mathrm{E}-10$ \\
\hline $1.00 \mathrm{E}-15$ & $4.5963 \mathrm{E}-25$ & $7.4716 \mathrm{E}-19$ \\
\hline
\end{tabular}

Table 25. Damping parameters versus pressure after using effective viscosity formula based on Knudsen number for air at $300^{\circ} \mathrm{K}$.

\subsubsection{Pressure Dependence}

The peak of displacement of microcantilever beam tip is plotted in Figure 102 versus driving frequencies corresponding to different pressures. There are 7 curves corresponding for 7 different pressures individually. From flat curve to sharp (bottom to top), the pressure are $1,1 / 3,0.1,0.01,10^{-3}, 10^{-4}$ and $10^{-5}$ atm. The last three curves $\left(10^{-3}\right.$, $10^{-4}$ and $10^{-5} \mathrm{~atm}$ ) are very similar to each other and cannot be clearly distinguished in Figure 102. Clearly, however, the value of $\delta_{\max }$ is more realistic than the earlier version using constant viscosity for all pressure values (see Table 25 in terms of peak 
displacement $\left.\delta_{\max }\right)$. As expected, the peak resonant frequency shifts to lower frequency as the pressure increases and the sharpness of the peak increases as the pressure decreases. Another indication that these results are reasonable can be found in the experimental data from Yang [42], shown in Figure 103 for lock-in amplifier signal amplitude, which looks quite similar in shape and character to the displacement data. The code of Matlab which plot the figure below is listed in Appendix D.

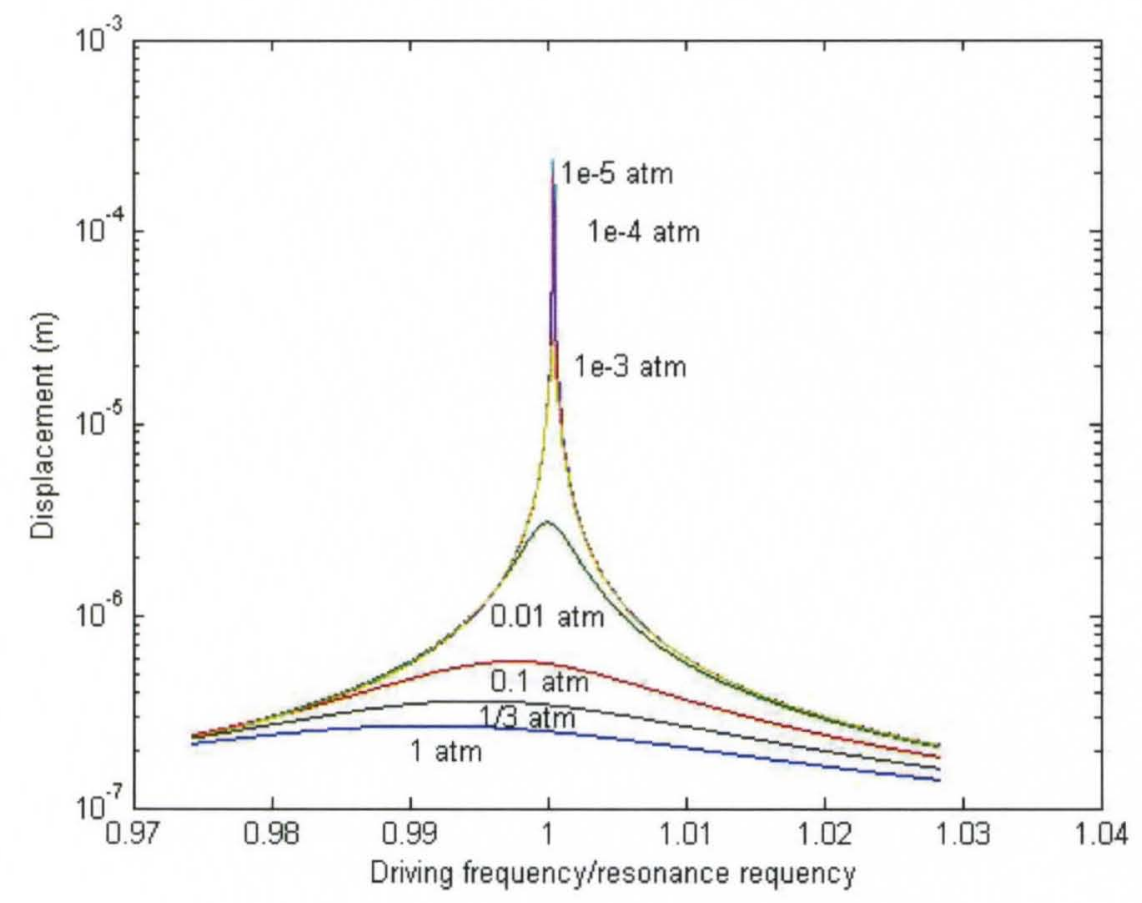

Figure 102. Peak amplitude of displacement vs. frequency with pressure as parameter. 


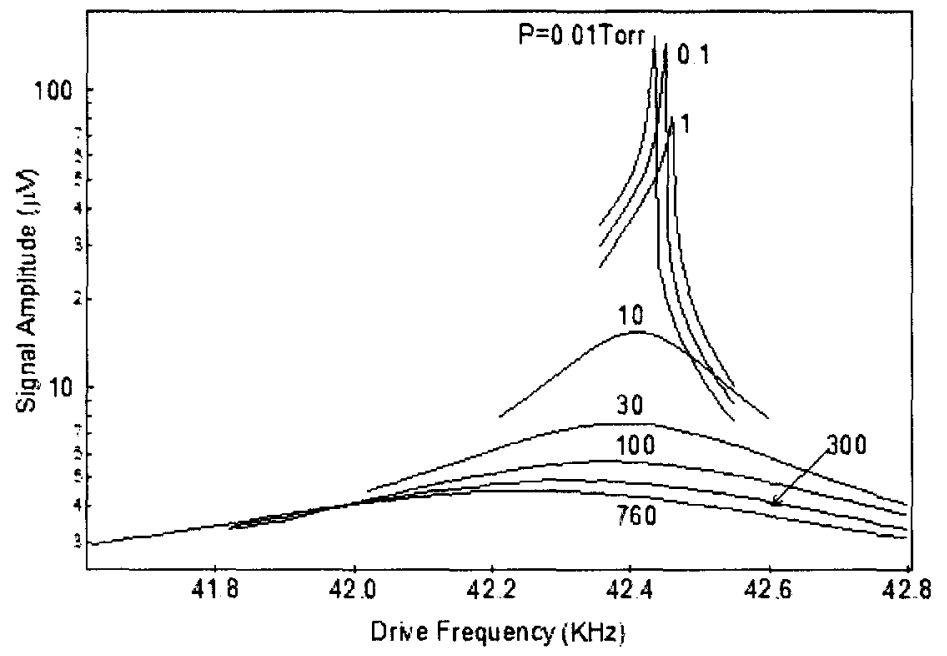

Figure 103. Response curve for different pressures from Yang's experiment data. [42] 


\section{CHAPTER 6. SIMULATION OF SIGNAL AT LOCK-IN AMPLIFIER}

From all of the work done in the previous chapters, the voltage signal can be derived provided to the lock-in amplifier during beam sensor operation can be simulated. In this section, the derivation of relationship between electrostatic force, beam displacement, change of resistance at the base, and the voltage signal that goes to the lock-in amplifier is discussed in detail.

\subsection{Summary of the Relationship Developed}

The relationship between electrostatic force and displacement of beam tip was discussed in Section 5.2, and the Equation (123) shows as:

$$
\begin{gathered}
u=c_{00}+c_{10} \sin (\omega t)+c_{11} \cos (\omega t)+c 20 \sin (2 \omega t)+c 21 \cos (2 \omega t) \\
\left.\left.\frac{F_{1} L^{3}}{3 E I}+\sum_{j=0}^{\infty} \phi_{j}(x)\left[K_{2 j} \cos \omega t+M_{2 j} \sin \omega t\right)\right]+\sum_{j=0}^{\infty} \phi_{j}(x)\left[K_{3 j} \cos \omega t+M_{3 j} \sin \omega t\right)\right] \\
F=k h_{0}+k h_{1} \sin (\omega t)-k h_{2} \cos (2 \omega t)
\end{gathered}
$$

The relationship between displacement of beam tip and change of resistance of the beam base was discussed in Section 2.6. The normalized changes of resistance for symmetric base configuration and asymmetric base configuration (with a leg separate gam of $2 \mu \mathrm{m}$ )are expressed by Equation (9) and Equation (10), respectively. 


$$
\begin{aligned}
& R_{S}(u)=\left(8.610 \times 10^{-9} u\right)+\left(-4.641 \times 10^{-8} u^{2}-8.233 \times 10^{-11}|u|\right) \\
& R_{A}(u)=\left(0.6427 \times 10^{-4} u\right)+\left(-1.901 \times 10^{-8} u^{2}-8.922 \times 10^{-12}|u|\right)
\end{aligned}
$$

where $u$ is the tip displacement (in $\mu \mathrm{m}$ ). The relationship between change of resistance of beam base and voltage signal was discussed in Section 2.5. The Equation (5) is expressed as:

$$
\begin{gathered}
\frac{\Delta V_{l}}{V_{D C}}=\frac{\mu}{(1+\mu)^{2}} \psi \quad \text { where } \quad \mu=\frac{R_{f}}{R_{b}} \text { and } \psi=\frac{\Delta R_{b}}{R_{b}} \\
R=R_{b}(1+\mu+\psi)
\end{gathered}
$$

where $\psi$ is appropriate normalized beam base resistance change (either $R_{S}$ or $R_{A}$, depending on the beam base configuration).

\subsection{Simulation of the Signal to the Lock-In Amplifier}

Using the relationships expressed above, the signal in lock-in amplifier can be determined. One case is considered below in detail, corresponding to a driving electrode voltage of $10 \sin \omega t$, a beam voltage of $5 \mathrm{~V}$ and the beam operating in air at $1 \mathrm{~atm}$. The tip displacement $u$ will be periodic with time duration $2 \pi / \omega$; this displacement can be provided to the obtain the change in beam base resistance over the same period $\left(\mathrm{R}_{\mathrm{S}}\right.$ or $\mathrm{R}_{\mathrm{A}}$ ), which in turn allows the change in the lock-in amplifier signal $\Delta \mathrm{V}_{\mathrm{L}}$ (i.e. the entire voltage minus the constant value corresponding to 0 beam tip displacement) to be determined. This signal can then be considered in term of optimal lock-in amplifier 
settings, especially whether the "1f" or " $2 \mathrm{f}$ " button (or some other arrangement) is most appropriate for the given experimental configuration.

The peak displacement of beam tip run as a function of driving electrode frequency is shown in by frequency sweep, and shown that the microcantilever beam resonating at both frequencies of $\omega$ and $2 \cdot \omega$. The reason for the peak at $\omega=\omega_{n 1} / 2$ was previously been demonstrated in Section 5.1 .5 as being due to the component of the driving force that contains the $\cos (2 \cdot \omega \cdot t)$ term.

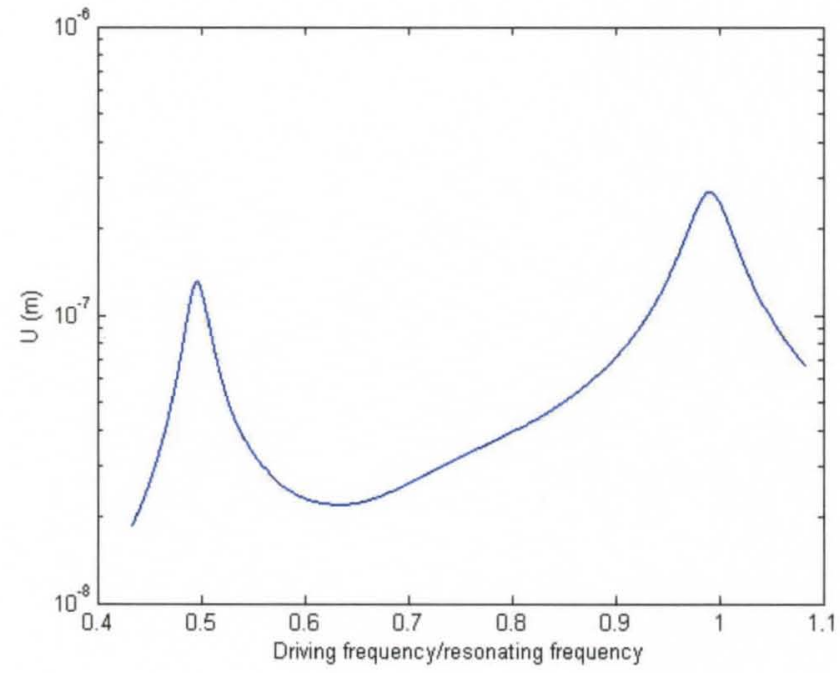

Figure 104. Peak displacement during a cycle as a function of driving electrode frequency (normalized by $\omega_{\mathrm{n} 1}=92.378 \mathrm{kHz}$ ) for $\mathrm{V}_{\mathrm{E}}=10$ and $\mathrm{V}_{\mathrm{B}}=5$.

\section{$\underline{6.2 .1 \text { Simulation at } 1 \mathrm{~atm}}$}

Both symmetric and asymmetric beam base are considered below for a beam operating in air at $1 \mathrm{~atm}$. Because the maximum change in voltage provided for the lockin amplifier $\left(\Delta V_{L}\right)$ occurs when $R_{b}=R_{f}$, it is assumed below that $R_{b}=R_{f}=14 k \Omega$. 
The system driven by frequency of $\omega$ is first considered. The electrostatic force $F(t)$, displacement of beam tip $u(t)$, the normalized change of beam base resistance $R_{S}(t)$ and $\mathrm{R}_{\mathrm{A}}(\mathrm{t})$, and the voltage provided to the lock-in amplifier for the symmetric $\left(\Delta \mathrm{V}_{\mathrm{LS}}\right)$ and asymmetric $\left(\Delta \mathrm{V}_{\mathrm{LA}}\right)$ cases are are plotted in Figure 105,Figure 106, Figure 107,Figure 108,Figure 109, and Figure 110, respectively, for a single driving electrode cycle period when operating at $\mathrm{f}=92.378 \mathrm{kHz}$. For this driving frequency data at $\mathrm{f}=92.378 \mathrm{kHz}$, the frequency of displacement the voltage at the lock-in amplifier is at the same frequency, although the symmetric base has some components that also appear to be at $2 \mathrm{f}$.

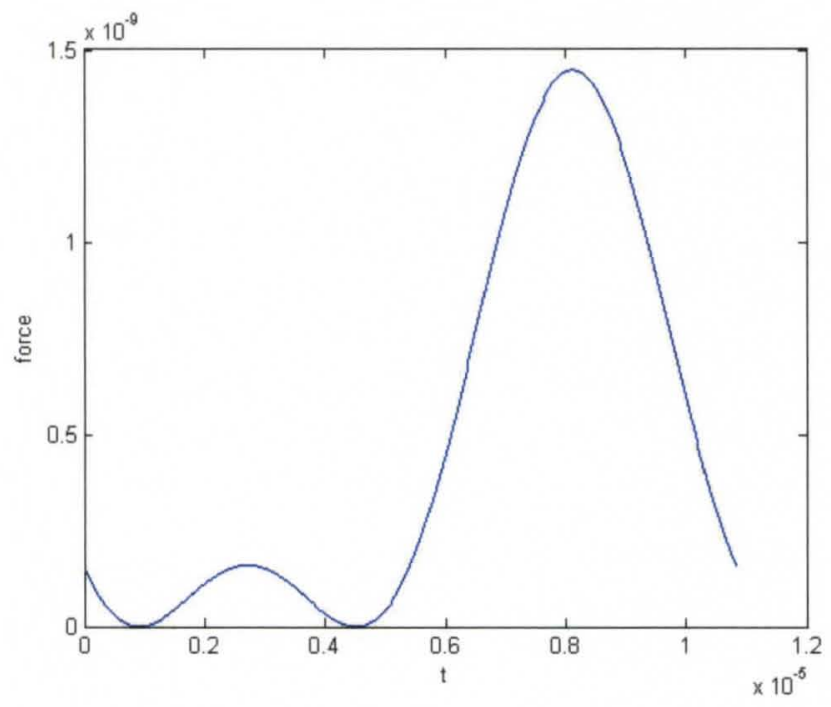

Figure 105. Electrostatic force (in $\mathrm{N}$ ) versus time for $\mathrm{f}=\mathrm{f}_{\mathrm{n} 1}=92.378 \mathrm{kHz}$ 


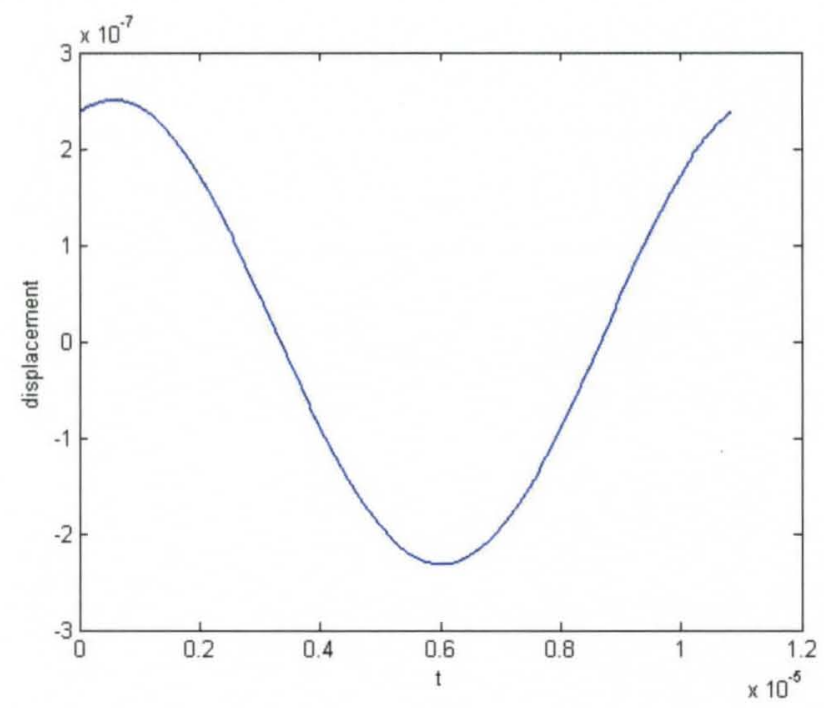

Figure 106. Displacement of beam tip (in $\mathrm{m}$ ) versus time for $\mathrm{f}=\mathrm{f}_{\mathrm{n} 1}=92.378 \mathrm{kHz}$.

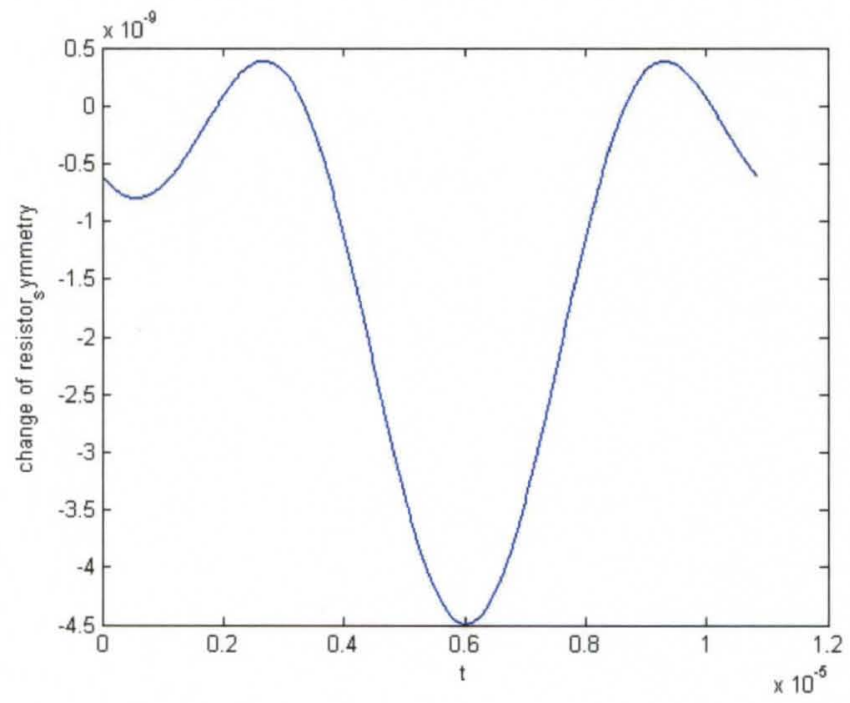

Figure 107. Change of normalized symmetric base resistance versus time for $f=f_{n 1}=$ $92.378 \mathrm{kHz}$. 


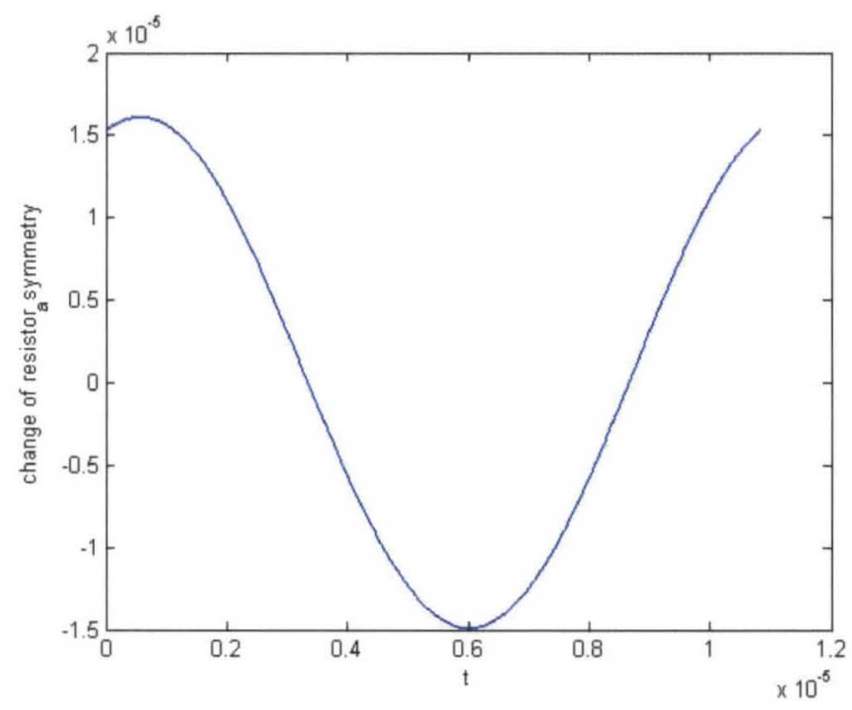

Figure 108. Change of normalized asymmetric beam base resistance versus time for $\mathrm{f}=$ $\mathrm{f}_{\mathrm{n} 1}=92.378 \mathrm{kHz}$.

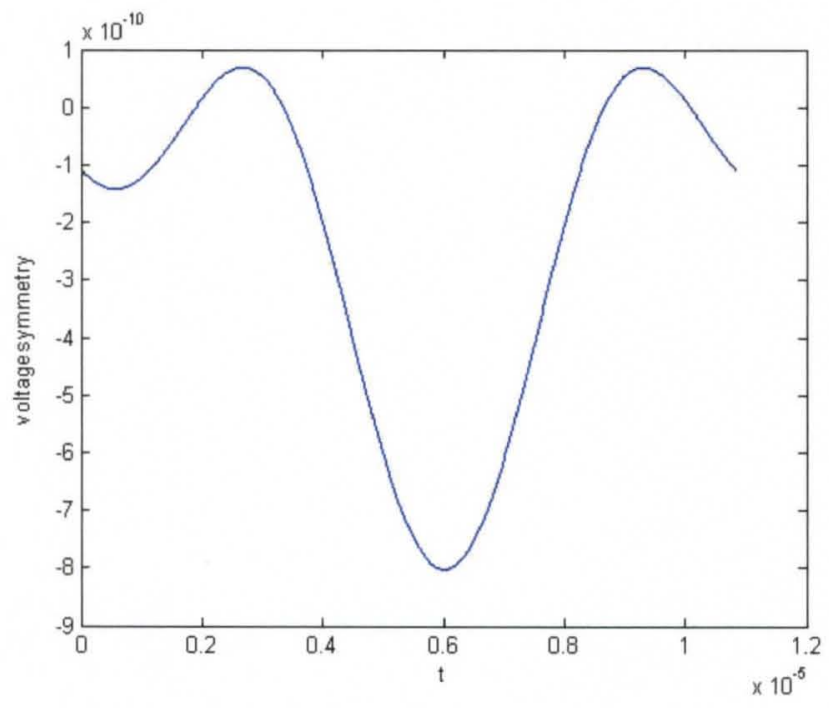

Figure 109. Voltage change measured by lock-in amplifier for symmetric beam base versus time for $\mathrm{f}=\mathrm{f}_{\mathrm{n} 1}=92.378 \mathrm{kHz}$. 


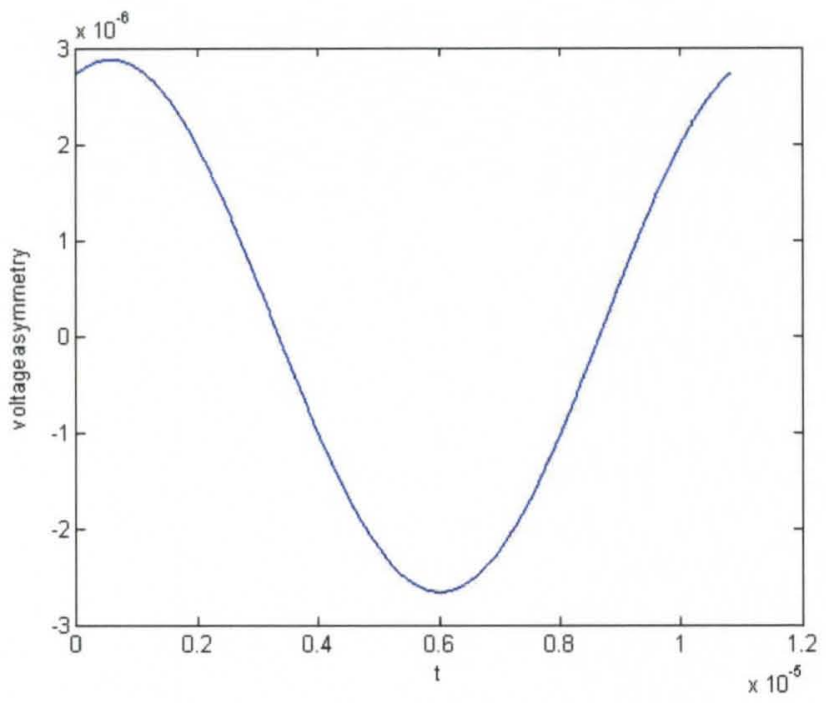

Figure 110. Voltage change measured by lock-in amplifier for asymmetric beam base versus time for $\mathrm{f}=\mathrm{f}_{\mathrm{n} 1}=92.378 \mathrm{kHz}$.

According the lock-in amplifier manual, the modal 5210 has limit frequency of internal oscillator which is from $5 \mathrm{~Hz}$ to $120 \mathrm{kHz}$. So if $2 \mathrm{f}$ is set in this test, the value of $2 \mathrm{f}$ is $184.756 \mathrm{kHz}$ which is bigger than the up limit of lock-in amplifier, no results will show up; therefore, only the " $1 \mathrm{f}$ " setting can be chosen for the lock-in amplifier in this case.

When system is driven by a driving electrode frequency $f=\left(\omega_{n 1} / 2\right) / 2 \pi$, the system can still can be resonated. The electrostatic force $F(t)$, displacement of beam tip $u(t)$, the normalized change of beam base resistance $R_{S}(t)$ and $R_{A}(t)$, and the voltage provided to the lock-in amplifier for the symmetric $\left(\Delta \mathrm{V}_{\mathrm{LS}}\right)$ and asymmetric $\left(\Delta \mathrm{V}_{\mathrm{LA}}\right)$ cases are plotted in Figure 105,Figure 111,Figure 112,Figure 113,Figure 114,Figure 115, and Figure 116 separately. Note that the duration of each plot is twice that of the previous set of plots since the driven frequency is half the earlier value; as such, the time window corresponds to 2 periods at frequency $f=\omega_{n 1} / 2 \pi$. In this case, the frequency of displacement of beam tip, change of resistors, and voltage of lock-in amplifier all at a 
frequency $f=\omega_{n 1} / 2 \pi$ or twice that of the driving frequency; the asymmetric base configuration also appears to include some components at twice this value (i.e. four times the driving frequency).

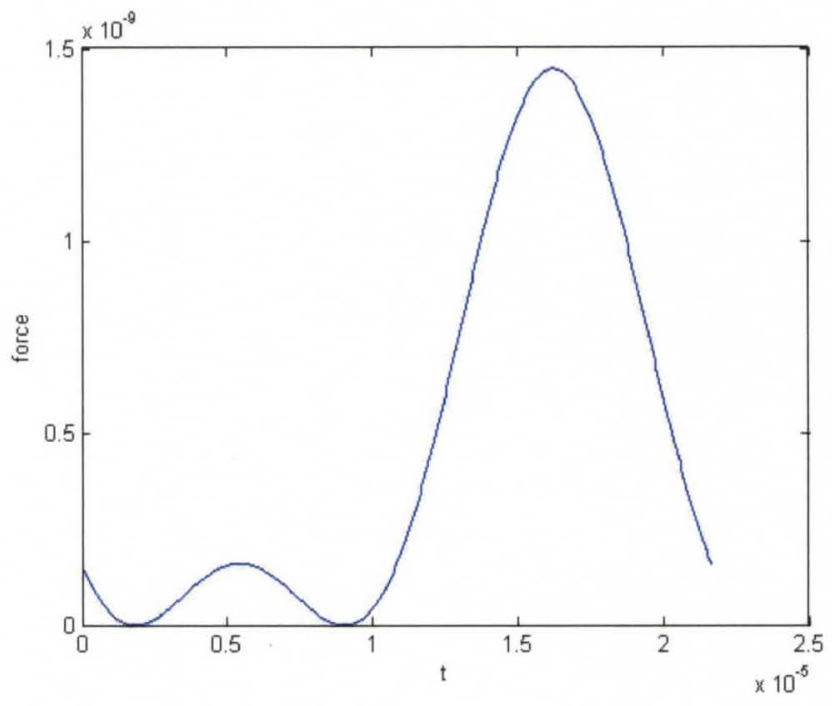

Figure 111. Electrostatic force versus time for $\mathrm{f}=1 / 2 \mathrm{f}_{\mathrm{n} 1}=36.189 \mathrm{kHz}$.

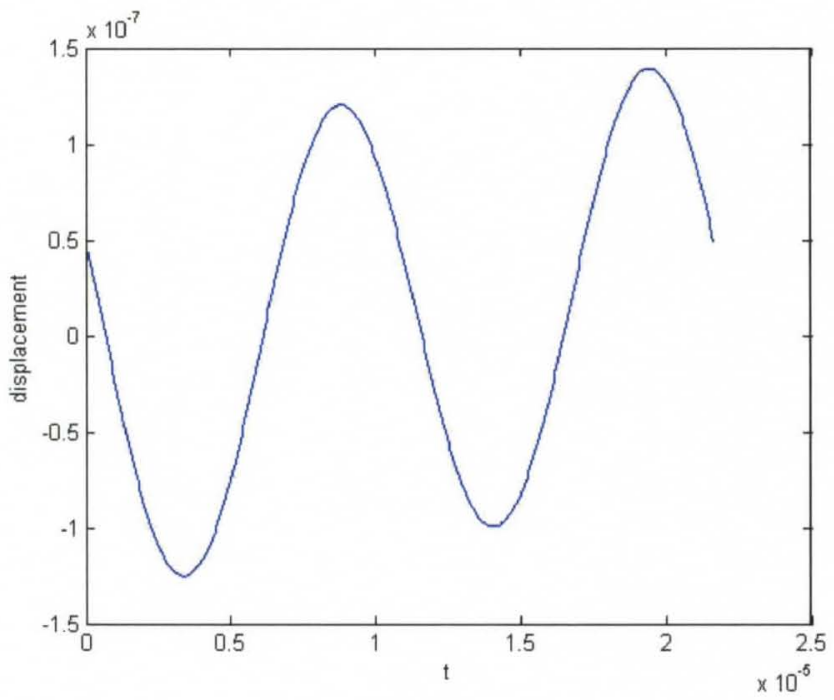

Figure 112. Displacement of beam tip versus time for $\mathrm{f}=1 / 2 \mathrm{f}_{\mathrm{n} 1}=36.189 \mathrm{kHz}$. 


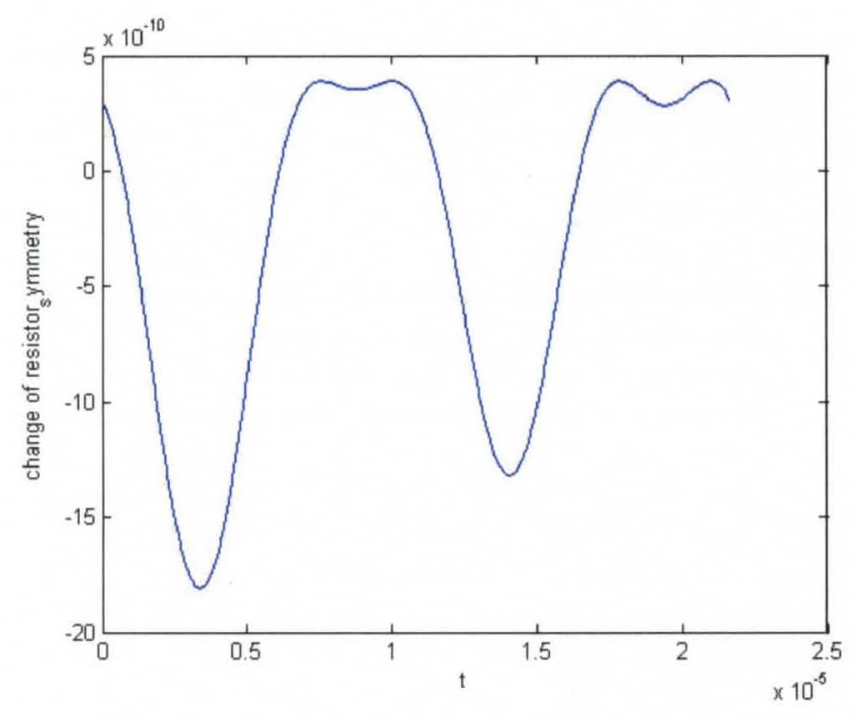

Figure 113. Change of normalized symmetric base resistance versus time for $f=1 / 2 f_{n 1}=$ $46.189 \mathrm{kHz}$.

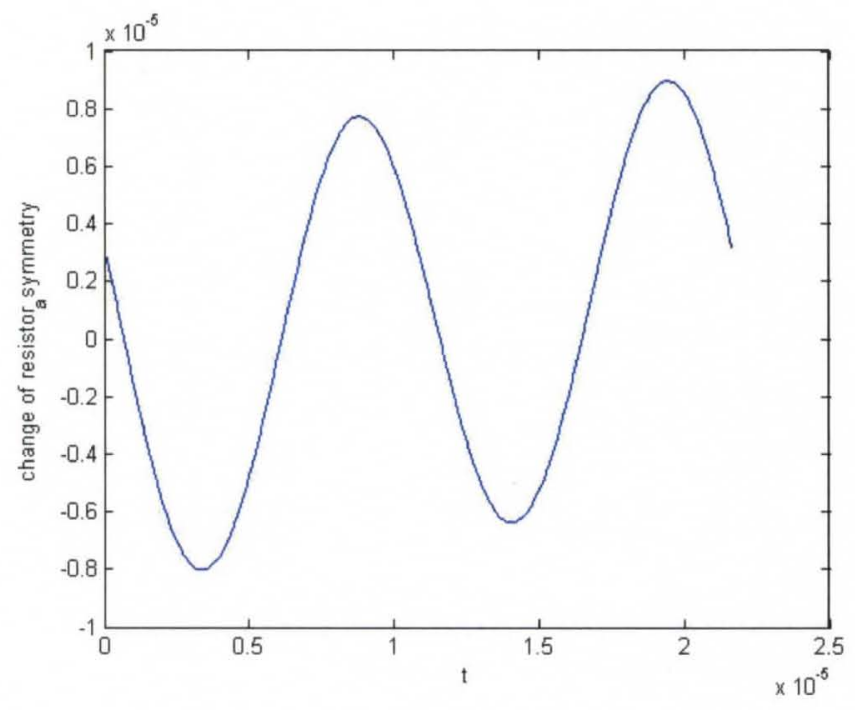

Figure 114. Change of normalized asymmetric base resistance versus time for $f=1 / 2 f_{n 1}=$ $46.189 \mathrm{kHz}$. 


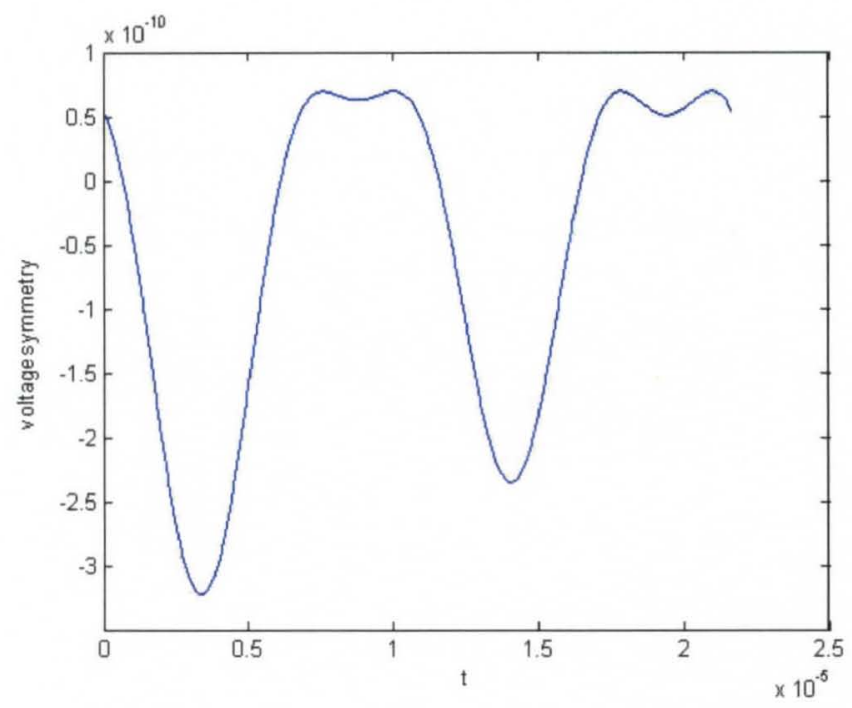

Figure 115 . Voltage change measured by lock-in amplifier for symmetric beam base versus time for $\mathrm{f}=1 / 2 \mathrm{f}_{\mathrm{n} 1}=46.189 \mathrm{kHz}$.

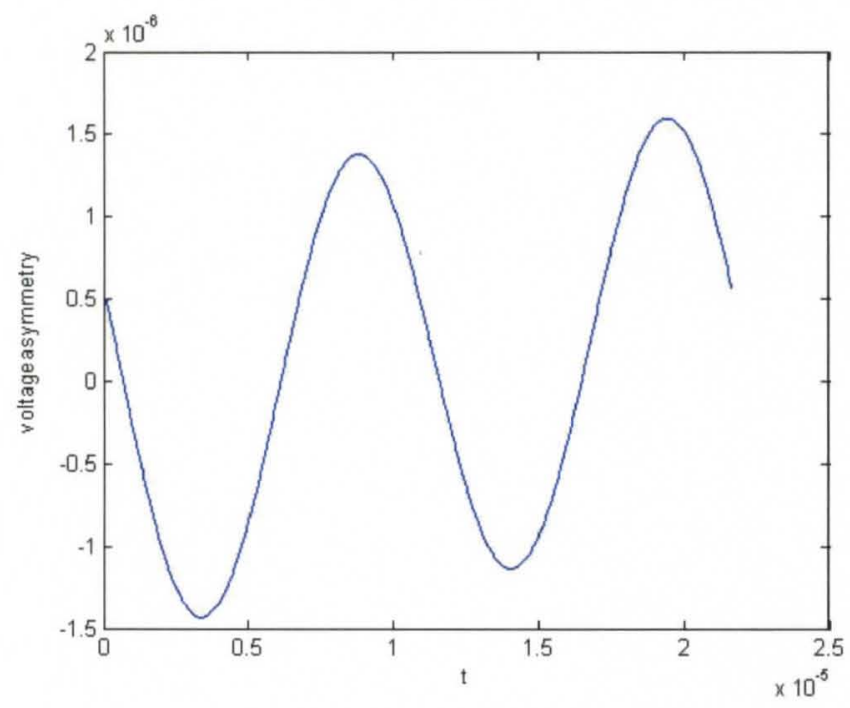

Figure 116 . Voltage change measured by lock-in amplifier for asymmetric beam base versus time for $\mathrm{f}=1 / 2 \mathrm{f}_{\mathrm{n} 1}=46.189 \mathrm{kHz}$.

Since the frequency of the signal received by the lock-in amplifier is double that of the driving (reference) frequency, the setting " $2 \mathrm{f}$ " must be set for lock-in amplifier for 
the signal to can be captured clearly. Alternately, if " $1 \mathrm{f}$ " is set, then no signal will be captured. This finding explains well why both the symmetric base and asymmetric base configurations must both use the " $2 \mathrm{f}$ " setting to capture the signal.

\section{$\underline{6.2 .2 ~ S i m u l a t i o n ~ a t ~} 1 \mathrm{E}-5 \mathrm{~atm}$}

The beam behavior is different due to the damping that exists in the earlier case. The calculations are now repeated to simulate the received lock-in amplifier voltage in the case of low damping forces due to the near-vacuum condition. The system response for both a driven frequency of $\mathrm{f}=\mathrm{f}_{\mathrm{n} 1}=92.368 \mathrm{kHz}$ (left side images) and a driven frequency of $\mathrm{f}=1 / 2 \mathrm{f}_{\mathrm{n} 1}=46.189 \mathrm{kHz}$ (right side figures). As in the earlier figures, the time duration is equal to one period of the driving electrode signal; therefore, the figures on the right are for twice the duration of those on the left.
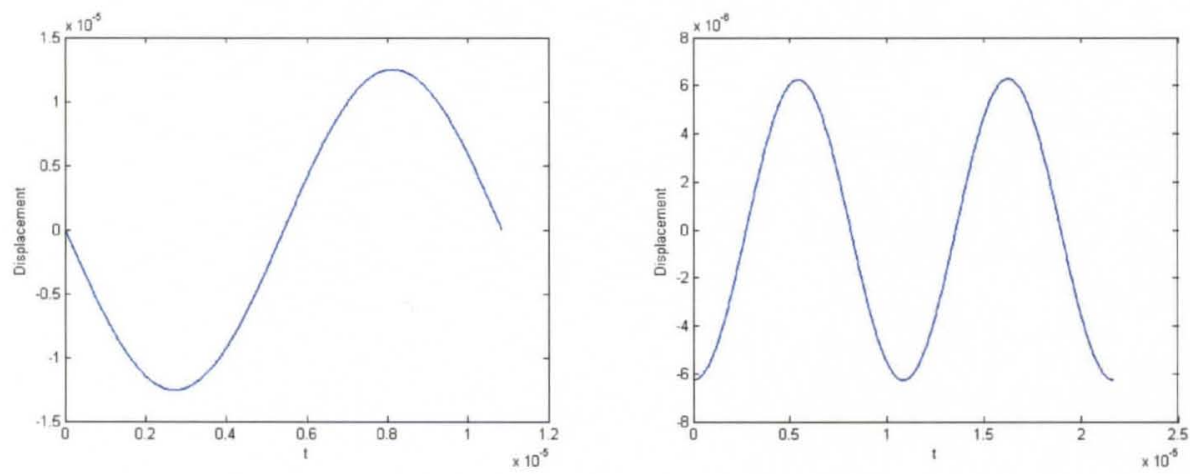

Figure 117. Displacement of beam tip versus time: $\left(\right.$ left) $f=f_{n 1}$; (right) $f=1 / 2 f_{n 1}$. 

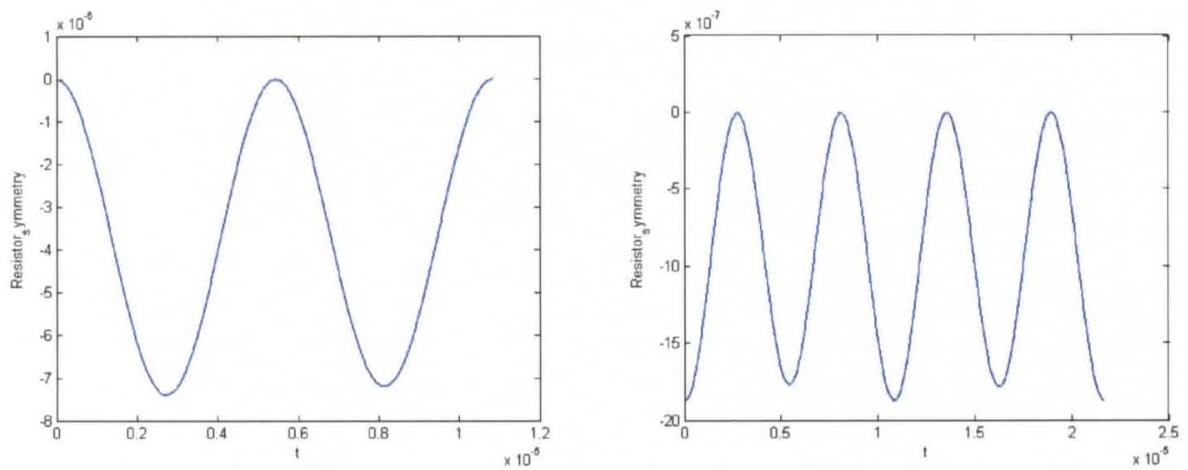

Figure 118. Change of normalized beam base resistance for symmetric base versus time:

$$
\text { (left) } f=f_{n 1} ; \text { (right) } f=1 / 2 f_{n 1} \text {. }
$$
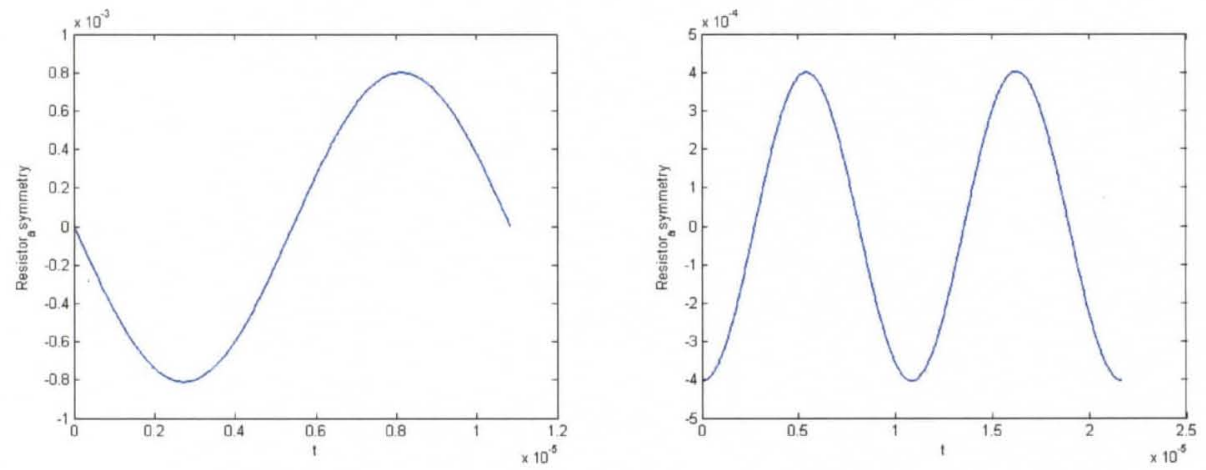

Figure 119. Change of normalized beam base resistance for asymmetric base versus time: (left) $f=f_{n 1}$; (right) $f=1 / 2 f_{n 1}$. 

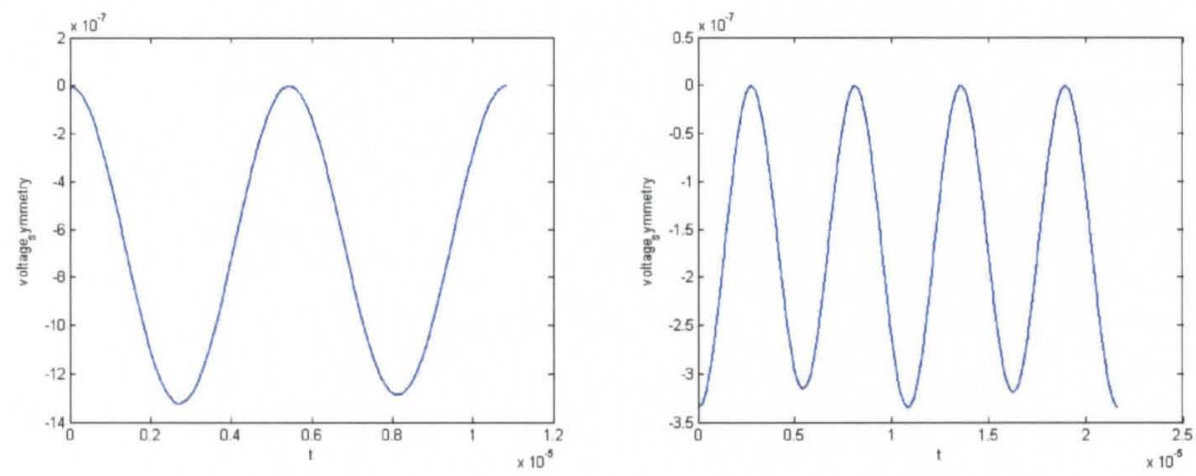

Figure 120. Voltage into lock-in amplifier for symmetric base versus time: (left) $f=f_{n 1}$; (right) $f=1 / 2 f_{n 1}$.
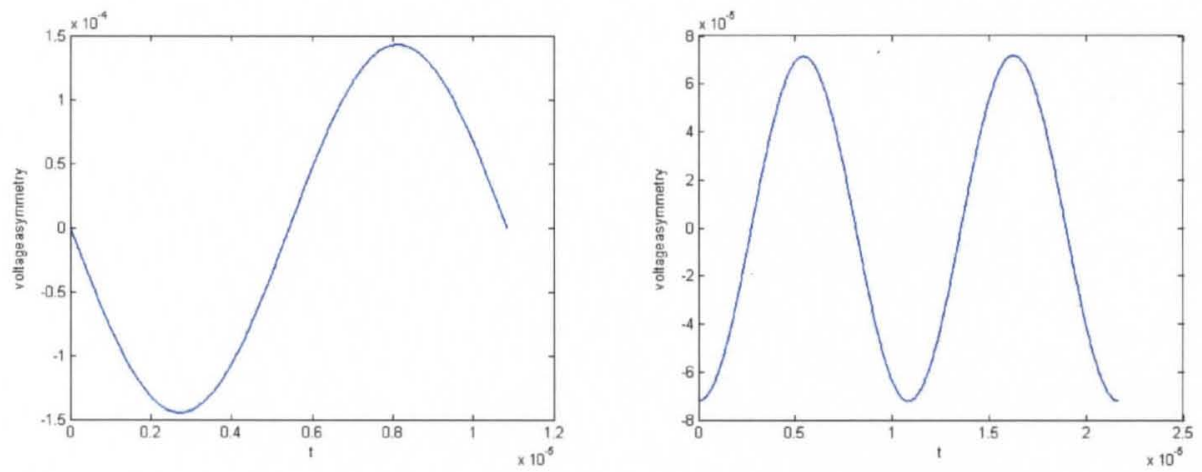

Figure 121. Voltage into lock-in amplifier for symmetric base versus time: (left) $f=f_{n 1}$; (right) $f=1 / 2 f_{n 1}$.

The results shows that in vacuum cases, the frequency voltage of asymmetric base is the same as driving frequency when input is $\omega$, and double when input is $\omega / 2$.

The frequency voltage of symmetric base is double of the driving frequency when driven by frequency of $\omega$. In this situation, the voltage signal could be captured by " $2 \mathrm{f}$ ". When driven frequency is changed to $\omega / 2$, the responding frequency is $2 \omega$ which equals 4 times driven frequency. 


\subsection{Conclusion}

This new understanding of the lock-in amplifier signal during resonance is an important finding. From the driving signal at the electrode, through electrostatic force, displacement of beam tip, change of resistor of base, then the signal transferred to the lock-in amplifier is estimated. This finding makes it clear why the signal can be found when the driving electrode is at the half of the resonance frequency of the beam only when the lock-in amplifier is set as ' $2 \mathrm{f}$ '. This provides a good foundation for further testing. 


\section{CHAPTER 7. CONCLUSION}

This dissertation describes several important research investigations into the use of piezoresistive microcantilevers beams as a gas sensor. This began with a new fabrication method that is beneficial in simplifying the approach and reducing the time required in the fabrication process. The new approach, while promising, also raised several new problems like width variation of the beams across the whole wafer and device leakage. The reason for those problems was investigated and thought to be due to the DRIE procedure, as the large exposed area of wafer may cause an overetch in DRIE. A new beam mask was designed as a possible way to fix the problem. Due to a shortage of both time and funds, the new mask has not been tested and this remains the subject of future work. The operation of the sensors in vacuum was shown to indicate that resonance is possible with devices made using the new approach.

Simulation using 2D computational fluid dynamics modeling has indicated that an accurate representation of fluid forces can be obtained under both steady state and oscillatory motion; this dissertation investigated into the effect of items of interest (beam cross-section, effect of handle layer below beam) to better understand the impact these have on the fluid forces. The forces for each case were calculated and compared to obtain a factor for the cross-section effect and the floor effect. These CFD efforts have been compared with the oscillating cylinder model developed by Stokes to predict the coefficients $c_{g}$ and $m_{g}$ to determine the drag force acting at the end of the resonating beam. The relationship between Stokes' theory value and CFD showed outstanding 
agreement. Therefore, the factors for the cross-section and floor effects can be applied to Stokes' model $\mathrm{m}_{\mathrm{g}}$ and $\mathrm{c}_{\mathrm{g}}$ values with confidence.

An understanding of the nature of the driving force for resonance (electrostatic attraction between the beam tip and the driving electrode) has also been modeled using finite element analysis. The response of the system to this load was then obtained. This provides a valuable insight into the likely reason why beams tend to vibrate when the driving electrode frequency is one half of the anticipated beam natural frequency. The behavior of a beam resonating in air at $92 \mathrm{kHz}$ is developed to show the beam motion with time. The damping effect characterization is studied and has the same character as data presented in previous research.

One important finding from the modeling effort relates to the voltage at the lockin amplifier. This work demonstrates clearly how the signal changes with time corresponding to the input voltage at the driving electrode and beam base. Those findings explained why the resonance signal is captured in the lock-in amplifier when the driving electrode is driven at one-half of the resonant frequency of the beam only using the " $2 \mathrm{f}$ " setting, regardless of the style of beam base configuration.

This research provides a good foundation to better understand the fabrication, modeling and operation of the piezoresistive microcantilever beams considered in this research study. It is anticipated that this work will foster the future development of gas sensors based on this technology. 


\section{REFERENCE}

1. Gfeller, K.Y., N. Nugaeva, and M. Hegner, Rapid Biosensor for Detection of Antibiotic-Selective Growth of Escherichia coli. Applied \& Environmental Microbiology, 2005. Vol. 71 ( Issue 5): p. p2626-2631.

2. Lavrik, N.V., M.J. Sepaniak, and P.G. Datsko, Cantilever transducers as a platform for chemical and biological sensors. Review of Scientific Instruments, 2004. 75(7): p. 2229-2253.

3. Lang, H.P., M. Hegner, and C. Gerber, Cantilever array sensors. Materials Today, 2005. 8(4): p. 30-36.

4. Chen, G.Y., et al., Resonance response of scanning force microscopy cantilevers. Review of Scientific Instruments, 1994. Vol. 65( Issue 8): p. 6p.

5. Gupta, A., D. Akin, and R. Bashir, Single virus particle mass detection using microresonators with nanoscale thickness. Applied Physics Letters, 2004. Vol. 84( Issue 11): p. p1976-1978.

6. Chen, G.Y., et al., Adsorption-induced surface stress and its effects on resonance frequency of microcantilevers. Journal of Applied Physics, 1995. 77(8): p. 3618.

7. Lang, H.P., et al., A chemical sensor based on a micromechanical cantilever array for the identification of gases and vapors. Applied Physics A: Materials Science \& Processing, 1998. 66(7): p. S61. 
8. Chen, G.Y., et al., Harmonic response of near-contact scanning force microscopy. Journal of Applied Physics, 1995. Vol. 78(Issue 3): p. 5p.

9. Shin 1, S., et al., Gas Sensor Application of Piezoelectric Cantilever Nanobalance; Electrical Signal Read-Out. Ferroelectrics, 2005. Vol. 328 (Issue 1): p. p59-65.

10. Bargatin, I., et al., Sensitive detection of nanomechanical motion using piezoresistive signal downmixing. Applied Physics Letters, 2005. 86(13): p. 133109.

11. Tortonese, M., R.C. Barrett, and C.F. Quate, Atomic resolution with an atomic force microscope using piezoresistive detection. Applied Physics Letters, 1993. 62(8): p. 834 .

12. Xu, Y., Fabrication, Development and Characterization of Micro/Nano Actuation Based Sensors and Applications for Gas Composition Analysis, in Department of Mechanical Engineering. 2006, University of Louisville: Louisville, KY.

13. Pinnaduwage, L.A., et al., Detection of 2,4-dinitrotoluene using microcantilever sensors. Sensors and Actuators B: Chemical, 2004. 99(2-3): p. 223-229.

14. Gotszalk, T., P. Grabiec, and I.W. Rangelow, Piezoresistive sensors for scanning probe microscopy. Ultramicroscopy, 2000. 82(1-4): p. 39-48.

15. Fletcher, P.C., Alternative piezoresistor designs for maximizing cantilever sensitivity, in Department of Mechanical Engineering. 2008, University of Louisville: Louisville, KY.

16. Fletcher, P.C., et al. Piezoresistive geometry for maximizing microcantilever array sensitivity. in IEEE Sensors. 2008. Lecce, Italy. 
17. Huang, X.M.H., et al., Nanomechanical hydrogen sensing. Applied Physics Letters, 2005. 86(14): p. 143104.

18. Kleimann, P., J. Linnros, and R. Juhasz, Formation of three-dimensional microstructures by electrochemical etching of silicon. Applied Physics Letters, 2001. 79(11).

19. Harley, J.A. and T.W. Kenny, High-sensitivity piezoresistive cantilevers under 1000 A thick. Applied physics letters, 1999. VOL.75.

20. Lin, R.M. and W.J. Wang, Structural dynamics of microsystems--current state of research and future directions. Mechanical Systems and Signal Processing, 2006. 20(5): p. 1015-1043.

21. Lin, R.M. and W.J. Wang, Structural dynamics of microsystems-current state of research and future directions. Mechanical Systems and Signal Processing, 2006. 20(5): p. 1015-1043.

22. Blom, F.R., et al., Dependence of the quality factor of micromachined silicon beam resonators on pressure and geometry. Journal of Vacuum Science \& Technology B: Microelectronics and Nanometer Structures, 1992. 10(1): p. 19-26.

23. Xu, Y., et al., Viscous damping of microresonators for gas composition analysis. Applied Physics Letters, 2006. 88(14): p. 143513.

24. Chon, J.W.M. and P. Mulvaney, Experimental validation of theoretical models for the frequency response of atomic force microscope cantilever beams immersed in fluids. Journal of Applied Physics, 2000. Vol. 87 (Issue 8): p. 11 p. 
25. Dufour, I.d. and L. Fadel, Resonant microcantilever type chemical sensors: analytical modeling in view of optimization. Sensors \& Actuators B: Chemical, 2003. Vol. 91( Issue 1-3): p. 9p.

26. Dufour, I.d.i.f., S.M.s.h.m.e. Heinrich, and F.f.j.m.e. Josse, Theoretical Analysis of Strong-Axis Bending Mode Vibrations for Resonant Microcantilever (Bio)Chemical Sensors in Gas or Liquid Phase. Journal of Microelectromechanical Systems, 2007. Vol. 16 (Issue 1): p. p44-49.

27. Gorman, D.G.d.g.s.a.u., et al., Analytical modelling and extraction of the modal behaviour of a cantilever beam in fluid interaction. Journal of Sound \& Vibration, 2007. Vol. 308(Issue 1/2): p. p231-245.

28. Green, C.P. and J.E. Sader, Torsional frequency response of cantilever beams immersed in viscous fluids with applications to the atomic force microscope. Journal of Applied Physics, 2002. 92(10): p. 6262-6274.

29. Jana, A., et al., Microcantilever mechanics in flowing viscous fluids. Applied Physics Letters, 2007. Vol. 90 (Issue 11): p. p114110-1.

30. Kaabi, L., et al., Modelling and analysis of MEMS sensor based on piezoresistive effects. Materials Science \& Engineering: C, 2007. 27(4): p. 691-694.

31. Ma, R.-H., et al., A microcantilever-based gas flow sensor for flow rate and direction detection. Microsystem Technologies, 2009. 15( 8): p. 1201-1205.

32. Weigert, S., M. Dreier, and M. Hegner, Frequency shifts of cantilevers vibrating in various media. Applied Physics Letters, 1996. Vol. 69 (Issue 19): p. p2834.

33. Van Eysden, C.A. and J.E. Sader, Frequency response of cantilever beams immersed in viscous fluids with applications to the atomic force microscope: 
Arbitrary mode order. Journal of Applied Physics, 2007. Vol. 101 (Issue 4,): p. p044908-N.PAG, 11p.

34. Yang, X., Fabrication, development and characterization of micro/nano actuation based sensors and applications for gas composition analysis. Dissertation, 2006.

35. Bradshaw, R.D., et al., Model Optimization of a Piezoresistive MicroactuationBased Sensor for Gas Composition Analysis. 2007.

36. Fletcher, P.C., Alternative Piezoresistor Designs for Mazimizing cantilever Sensitivity. University of Louisville, 2008.

37. Bradshaw, R.D., et al., Model Optimization of a Piezoresistive MicroactuationBased Sensor for Gas Composition Analysis, in 2007 Joint ASME/ASCE/SES Conference on Mechanics and Materials. 2007: Austin, TX.

38. Wikipedia. Microelectromechanical systems. Available from: http://en.wikipedia.org/wiki/Microelectromechanical_systems.

39. MEMS, A., Fabricating MEMS and Nanotechnology.

40. Liu, H.C., Y.H. Lin, and W. Hsu, Sidewall roughness control in advanced silicon etch process. Microsystem Technologies, 2003. 10(1): p. 29-34.

41. Fang, S. and J.P. McVittie, Oxide damage from plasma charging: breakdown mechanism and oxide quality. IEEE Transactions on Electron Devices, 1994. 41(6): p. 1034-1039.

42. Yang, X., Fabrication, development and characterization of micro/nano actuation based sensors and applications for gas composition analysis, in Dissertation. 2006 .

43. Holman, J.P., Heat Transfer. Book. 2010. 4. 
44. Young, M. and O. Huebsch, Fundamentals of Fluid Mechanics. Book. 2009. 308318.

45. Taneda, S., Visualization of separating Stokes flows. The Physical Society of Japan, 1979. 46(6): p. 7.

46. Panton, R.L., Imcompressible flow. BOOK. 1984. 251-254.

47. Stokes, On the effect of the internal friction of fluids on the motion of pendulums. Transactions of the Cambridge Philosophical Society, 1850. Vol. IX: p. 8.

48. Lamb, ed. Hydrodynamics. 1945. 642-645.

49. Stokes, G.G., On the effect of the internal friction of fluids on the motion of pendulums. Transactions of the Cambridge Philosophical Society, 1850. Vol. IX: p. 8 .

50. Cook, R.D., et al., Concepts and Applications Of Finite Element Analysis. 2002. $238-240,552$.

51. Bao, M., Analysis and Design Principles of MEMS Devices. BOOK. 2005. 3.

52. Schmidt, J.W. and M.r. Moldover, Dielectric Permittivity of International Journal of Thermophysics, 2003. Vol.24(No.2): p. 375-403 (29).

53. Berli, C.L.A. and A. Cardona, On the calculation of viscous damping of microbeam resonators in air. Journal of Sound and Vibration, 2009. 327(1-2): p. 249-253.

54. Rao, S.S., Mechanical Vibrations. Vol. 8. 2003. 588-655.

55. Kreyszig, E., Advanced Engineering Mathematics. BOOK. 1999. 115.

56. Cook, R.D., Finite Element Modeling for Stress Analysis, in Book. 1995.

57. Bird, R.B., W.E. Stewart, and E.N. Lightfoot, Transport Phenomena Book. 2006. 
58. Michalis, V.K., et al., Rarefaction effects on gas viscosity in the Knudsen transition regime. Micorfluidics and Nanofluidics, 2010. 9(4-5): p. 847-853. 


\begin{abstract}
APPENDIX A
This Appendix contains measurements made in the SEM for beams on the devices indicated. A narrative of the testing history is also provided below.
\end{abstract}

\begin{tabular}{|c|c|c|c|}
\hline width1\# & $1.1 \mu \mathrm{m}$ & $2 \#$ & $1.3 \mu \mathrm{m}$ \\
\hline beam 1 & 0.6767 & beam 1 & broken \\
\hline beam 2 & 0.7181 & beam 2 & 1.045 \\
\hline beam 3 & 0.7507 & beam 3 & 1.054 \\
\hline beam 4 & 0.6891 & beam 4 & 0.9805 \\
\hline beam 5 & 0.6891 & beam 5 & 0.9805 \\
\hline beam 6 & 0.635 & beam 6 & broken \\
\hline beam 7 & 0.7234 & beam 7 & 0.9534 \\
\hline beam 8 & 0.5603 & beam 8 & 1.036 \\
\hline beam 9 & 0.5293 & beam 9 & 1.095 \\
\hline beam 10 & base broken & beam 10 & 1.095 \\
\hline
\end{tabular}

\begin{tabular}{|c|c|c|c|}
\hline $3 \#$ & $1.3 \mu \mathrm{m}$ & $\# 4$ & $1.1 \mu \mathrm{m}$ \\
\hline beam 1 & 0.7592 & beam 1 & 0.9359 \\
\hline beam 2 & 0.806 & beam 2 & 0.9896 \\
\hline beam 3 & 0.567 & beam 3 & 0.9805 \\
\hline beam 4 & 0.7062 & beam 4 & base broken \\
\hline beam 5 & 0.7848 & beam 5 & base broken \\
\hline beam 6 & 0.7848 & beam 6 & 0.7937 \\
\hline beam 7 & 0.7952 & beam 7 & 0.9338 \\
\hline beam 8 & broken & beam 8 & 0.8863 \\
\hline beam 9 & broken & beam 9 & 0.9481 \\
\hline beam 10 & 0.551 & beam 10 & 1.045 \\
\hline
\end{tabular}




\begin{tabular}{|c|c|c|c|}
\hline$\# 5$ & $1.7 \mu \mathrm{m}$ & $\# 6$ & $1.1 \mu \mathrm{m}$ \\
\hline beam 1 & 0.9719 & beam 1 & 0.9178 \\
\hline beam 2 & 1.021 & beam 2 & 0.8146 \\
\hline beam 3 & 0.8484 & beam 3 & broken \\
\hline beam 4 & 1.028 & beam 4 & broken \\
\hline beam 5 & 0.893 & beam 5 & 0.7005 \\
\hline beam 6 & 0.9907 & beam 6 & 0.8923 \\
\hline beam 7 & 0.9641 & beam 7 & 0.8137 \\
\hline beam 8 & 1.07 & beam 8 & 0.9133 \\
\hline beam 9 & 0.9181 & beam 9 & 0.9016 \\
\hline beam 10 & 0.7056 & beam 10 & 0.9164 \\
\hline
\end{tabular}

\begin{tabular}{|c|c|c|c|}
\hline$\# 7$ & $1.7 \mu \mathrm{m}$ & $\# 8$ & $1.3 \mu \mathrm{m}$ \\
\hline beam 1 & 0.5464 & beam 1 & broken \\
\hline beam 2 & 0.5056 & beam 2 & 1.061 \\
\hline beam 3 & 0.4675 & beam 3 & 1.039 \\
\hline beam 4 & 0.5862 & beam 4 & 1.039 \\
\hline beam 5 & 0.6208 & beam 5 & 0.8853 \\
\hline beam 6 & 0.5549 & beam 6 & broken \\
\hline beam 7 & 0.5826 & beam 7 & 0.9558 \\
\hline beam 8 & 0.553 & beam 8 & 0.9094 \\
\hline beam 9 & 0.5761 & beam 9 & 1.008 \\
\hline beam 10 & 0.7381 & $\begin{array}{c}\text { beam } \\
10\end{array}$ & 1.008 \\
\hline
\end{tabular}

\begin{tabular}{|c|c|c|c|}
\hline$\# 9$ & $2.1 \mu \mathrm{m}$ & $\# 11$ & $1.1 \mu \mathrm{m}$ \\
\hline beam 1 & 0.6589 & beam 1 & $\begin{array}{c}\text { base } \\
\text { broken }\end{array}$ \\
\hline beam 2 & 0.6753 & beam 2 & 1.061 \\
\hline beam 3 & 0.8412 & beam 3 & 1.039 \\
\hline beam 4 & 0.7582 & beam 4 & 1.039 \\
\hline beam 5 & 0.8502 & beam 5 & 0.8853 \\
\hline beam 6 & 1.129 & beam 6 & broken \\
\hline beam 7 & 0.9157 & beam 7 & 0.9558 \\
\hline beam 8 & 1.044 & beam 8 & 0.9094 \\
\hline beam 9 & 1.099 & beam 9 & 1.008 \\
\hline $\begin{array}{c}\text { beam } \\
10\end{array}$ & 0.8952 & $\begin{array}{c}\text { beam } \\
10\end{array}$ & 1.008 \\
\hline
\end{tabular}




\begin{tabular}{|c|c|c|c|}
\hline$\# 12$ & $2.1 \mu \mathrm{m}$ & $\# 14$ & $1.3 \mu \mathrm{m}$ \\
\hline beam 1 & broken & beam 1 & 0.7834 \\
\hline beam 2 & $\begin{array}{c}1.129 \text { base over } \\
\text { ethch }\end{array}$ & beam 2 & 0.7834 \\
\hline beam 3 & 1.082 & beam 3 & 0.7719 \\
\hline beam 4 & 1.77 & beam 4 & 0.7477 \\
\hline beam 5 & 1.77 & beam 5 & broken \\
\hline beam 6 & 1.271 & beam 6 & 0.7203 \\
\hline beam 7 & 1.238 & beam 7 & 0.7398 \\
\hline beam 8 & 1.204 & beam 8 & 0.7398 \\
\hline beam 9 & 1.736 & beam 9 & 0.6834 \\
\hline $\begin{array}{c}\text { beam } \\
10\end{array}$ & 1.821 & beam & 0.7477 \\
\hline
\end{tabular}

\section{Device Test History}

Device was tested in probe station for different purposes: 1) For each beam's resonating frequency; in this case, the beam's frequency was recorded and listed in the following tables. 2) Other tests like for signal analysis using oscilloscope, pressure leakage in probe station, leakage test for SO2 layer, DC effect and $\mathrm{AC}$ effect in frequency shift, and pressure effect etc. In these tests, a bunch of beams were put into tests, but the frequencies were not recorded clearly.

\begin{tabular}{|c|c|c|c|}
\hline & \multicolumn{3}{|c|}{ Wafer A } \\
\hline Unit: $\mathrm{kHz}$ & $1.3 \mu \mathrm{m}$ & $1.1 \mu \mathrm{m}$ & $1.3 \mu \mathrm{m}$ \\
\hline beam 1 & 56.89 & $\mathrm{x}$ & 32.47 \\
\hline beam 2 & 56.34 & 45.74 & $\mathrm{x}$ \\
\hline beam 3 & $\mathrm{x}$ & 36.8 & $\mathrm{x}$ \\
\hline beam 4 & 56.17 & 46.35 & 31.83 \\
\hline beam 5 & broken & electrode broken & 35.46 \\
\hline beam 6 & 35.8 & $\mathrm{x}$ & 38.5 \\
\hline beam 7 & $\mathrm{x}$ & 40.25 & 32.53 \\
\hline beam 8 & $\mathrm{x}$ & 35.83 & 33.18 \\
\hline beam 9 & 47.57 & 37.4 & 32.2 \\
\hline beam 10 & 54.31 & 38.8 & $\mathrm{x}$ \\
\hline
\end{tabular}




\begin{tabular}{|c|c|c|c|c|}
\hline & \multicolumn{4}{|c|}{ Wafer A } \\
\hline Unit: $\mathrm{kHz}$ & $1.3 \mu \mathrm{m}$ & $1.3 \mu \mathrm{m}$ & $1.1 \mu \mathrm{m}$ & \\
\hline beam 1 & $\mathrm{x}$ & $\mathrm{x}$ & $\mathrm{x}$ & broken \\
\hline beam 2 & 38.85 & 31.13 & $\mathrm{x}$ & 39.98 \\
\hline beam 3 & 36.18 & 30.11 & 36.7 & 35.94 \\
\hline beam 4 & $\mathrm{x}$ & 24.42 & $\mathrm{x}$ & broken \\
\hline beam 5 & $\mathrm{x}$ & $\mathrm{x}$ & 37.24 & broken \\
\hline beam 6 & $\mathrm{x}$ & $\mathrm{x}$ & 37.4 & 33.67 \\
\hline beam 7 & 34.89 & 28.35 & 36.13 & 32.4 \\
\hline beam 8 & 32.2 & $\mathrm{x}$ & 35.5 & 33.12 \\
\hline beam 9 & 33.2 & 70 & 33.2 & 33.2 \\
\hline beam 10 & 33.5 & 20.35 & broken & dust \\
\hline
\end{tabular}

\begin{tabular}{|c|c|c|}
\hline & \multicolumn{2}{|c|}{ Wafer B } \\
\hline Unit: $\mathrm{kHz}$ & $1.1 \mu \mathrm{m}$ & $1.1 \mu \mathrm{m}$ \\
\hline beam 1 & $\mathrm{x}$ & $\mathrm{x}$ \\
\hline beam 2 & $\mathrm{x}$ & $\mathrm{x}$ \\
\hline beam 3 & broken & $\mathrm{x}$ \\
\hline beam 4 & $\mathrm{x}$ & $\mathrm{x}$ \\
\hline beam 5 & stick & $\mathrm{x}$ \\
\hline beam 6 & 48.74 & 31.82 \\
\hline beam 7 & $\mathrm{x}$ & $\mathrm{x}$ \\
\hline beam 8 & $\mathrm{x}$ & $\mathrm{x}$ \\
\hline beam 9 & $\mathrm{x}$ & $\mathrm{x}$ \\
\hline beam 10 & $\mathrm{x}$ & $\mathrm{x}$ \\
\hline
\end{tabular}

Among 9 devices which were testing for natural frequency, the device from wafer B is hard to be resonated. The reason should be investigated. The devices from wafer A are mainly about 1.1 and $1.3 \mu \mathrm{m}$ of beam width. Most driving electrode frequencies are in the range of $30-40 \mathrm{kHz}$; however, some devices had as high a value as $55 \mathrm{kHz}$ and others had as low as $20 \mathrm{kHz}$. It should be noted that the $20 \mathrm{kHz}$ case was obtained accidently; it was tried after the beam resonated at $40 \mathrm{kHz}$. It is possible that other devices have a lower resonating frequency similar to this one; this was not tested in 
the interest of time. Another possibility is that $40 \mathrm{kHz}$ could represent the resonant frequency of the beam and then $20 \mathrm{kHz}$ was the second peak for driving at one-half the beam resonant frequency; this would be feasible if the beam width was well below the intended value (perhaps on the order of $0.6-0.7 \mu \mathrm{m}$ ). 


\section{APPENDIX B}

Below are images for future device masks

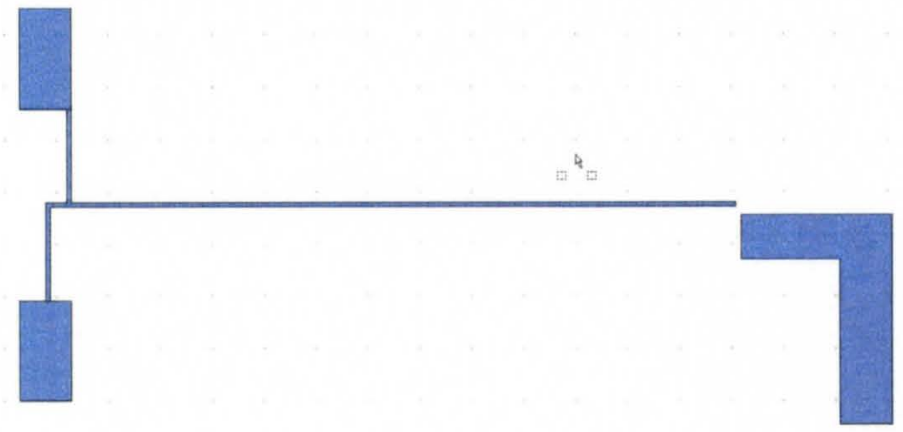

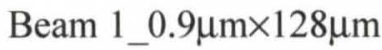

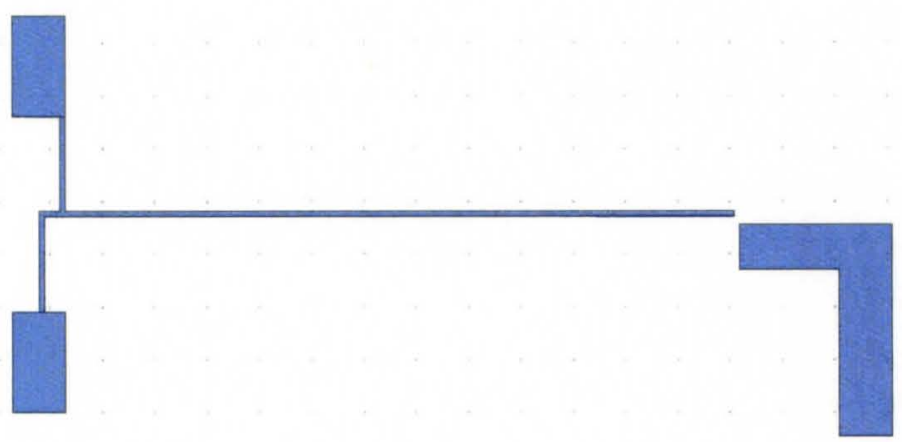

Beam 2_1.0 $\mu \mathrm{m} \times 128 \mu \mathrm{m}$

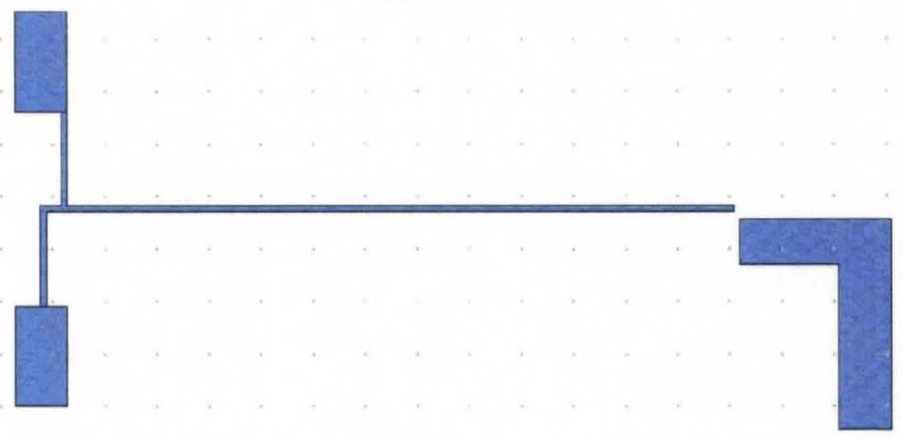

Beam 3,4,5_1.1 $\mu \mathrm{m} \times 128 \mu \mathrm{m}$ 

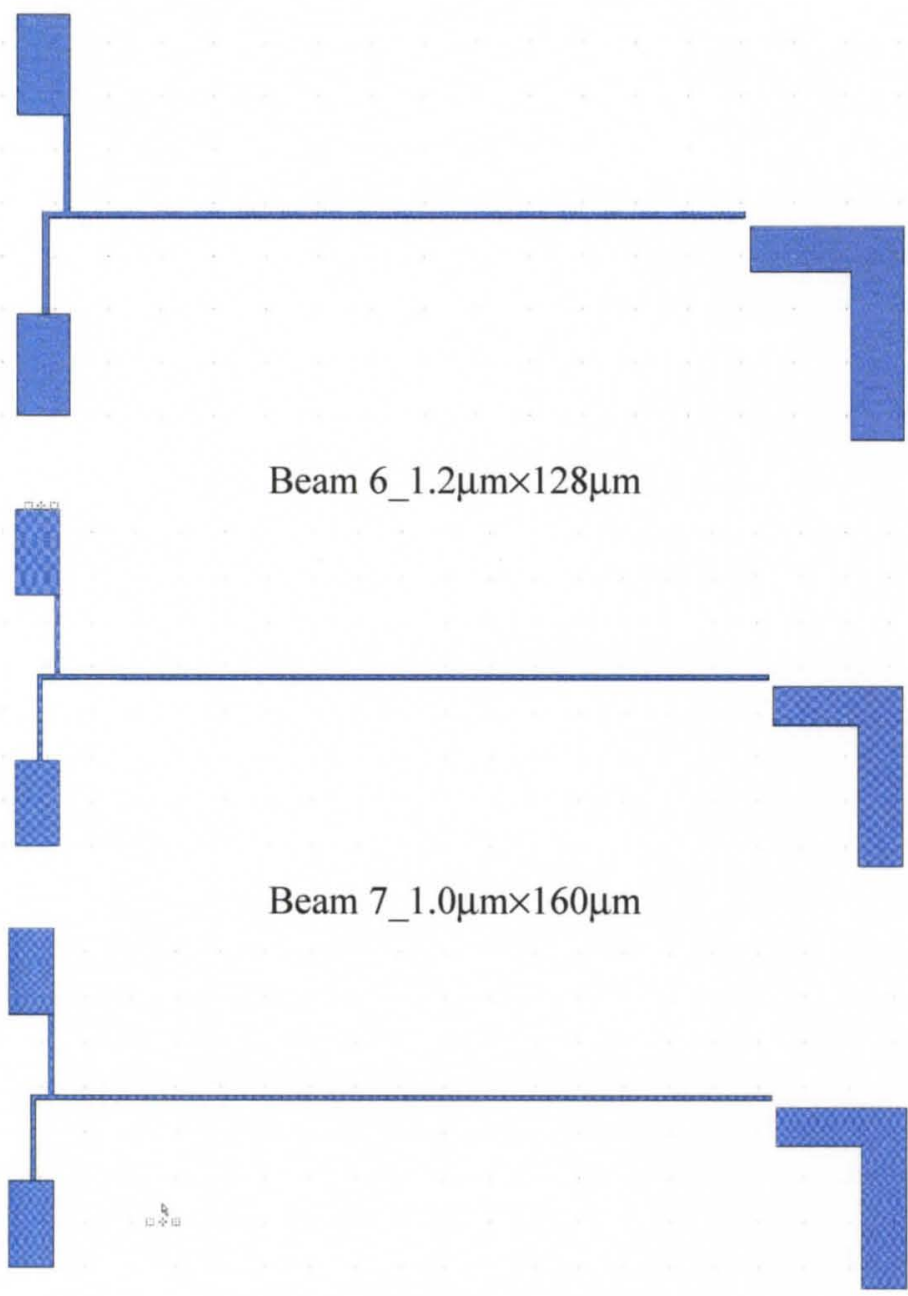

Beam 8,9_1.1 $\mu \mathrm{m} \times 160 \mu \mathrm{m}$

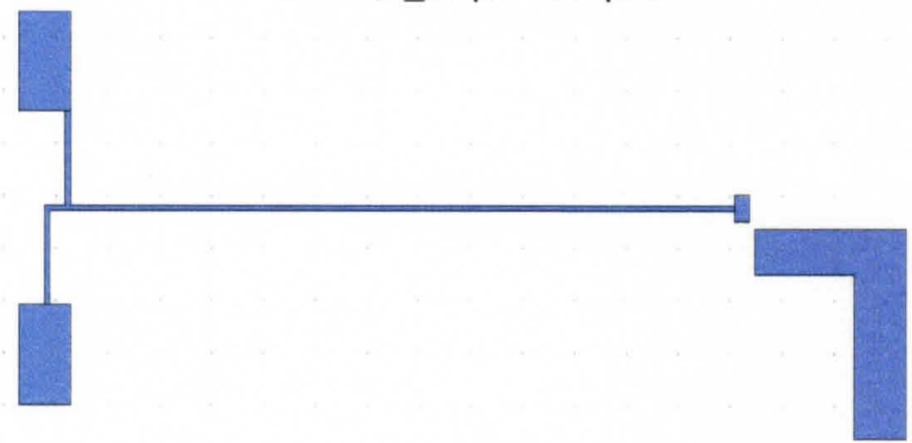

Beam 10_1.1 $\mu \mathrm{m} \times 128 \mu \mathrm{m} \_$tip mass 


\begin{abstract}
APPENDIX C
This section contains the MathCad document used to generate the Stokes oscillating cylinder model values for $\mathrm{k}$ and $\mathrm{k}$ ' as functions of parameter $\mathrm{m}$. The verification of the values as well as the details of the curve fit are presented. Each page of the MathCad printout is reproduced here as an embedded image.
\end{abstract}




\section{Calculation of Stokes' Model Parameters - Oscillating Cylinder}

Roger Bradshaw, 4 March 2011

Update, 28 November 2012 - Added Curve Fits

Update, 11 December 2012 - Dissertation Version

This document implements the Stokes oscillating cylinder model. The model and all scanned text below were taken from the paper "Stokes, G.G. On the effect of the internal friction of fluids on the motion of pendulums. Transactions of the Cambridge Philosophical Society, 1850. Vol. IX, p. 8", from http.I/www. nawcc-index. net/Articles/Stokes-InternalFriction. pdf A better reference is to use Stokes' collected papers: G. G. Stokes, Mathematical and Physical Papers, Vol. III. 1901. Cambridge at the University Press (accessed as digitized version from the University of Wisconsin volume)

$S_{i}$ - Equation (86)
$S(i):=\sum_{j=1}^{i} \frac{1}{j}$
$\mathrm{S}(1)=1$
$\mathrm{S}(2)=1.5$
$\mathrm{S}(3)=1.833$
$S(4)=2.083$

Power Advance Terms - these convert $j$ into appropriate powers for next equations
$p(j):=4 \cdot j-2$
$\mathrm{p}(1)=2$
$\mathrm{p}(2)=6$
$\mathrm{p}(3)=10$
$q(j):=4 \cdot j$
$q(1)=4$
$q(2)=8$
$\mathrm{q}(3)=12$

$\mathrm{MO}_{\mathrm{i}}$ - Equation (103) - This is one term where powers advance $2,6,10$, etc

$$
\begin{array}{clll}
\mathrm{MO}(\mathrm{m}, \mathrm{j}):=\frac{(-1)^{\mathrm{j}+1} \cdot \mathrm{m}^{\mathrm{P}(\mathrm{j})}}{\left(\prod_{\mathrm{k}=1}^{2 \cdot j-1} \mathrm{k}^{2}\right) \cdot(2 \cdot \mathrm{j}-1)^{-1}} \quad \mathrm{MO}(2,1)=4 & \mathrm{MO}(2,2)=-5.333 & \mathrm{MO}(2,3)=0.356 \\
\frac{2^{2}}{1}=4 & \frac{2^{6}}{2^{2} \cdot 3}=-5.333 & \frac{2^{10}}{2^{2} \cdot 3^{2} \cdot 4^{2} \cdot 5}=0.356
\end{array}
$$

MOp - Equation (103) - This is one term where powers advance 2, 6, 10, etc.

$$
\begin{array}{llll}
\operatorname{MOp}(m, j):=\frac{(-1)^{j+1} \cdot m^{p(j)}}{\left(\prod_{k=1}^{2 \cdot j-1} k^{2}\right)} & \operatorname{Mop}(2,1)=4 & \operatorname{MOp}(2,2)=-1.778 & \operatorname{MOp}(2,3)=0.071 \\
\frac{2^{2}}{1}=4 & \frac{2^{6}}{2^{2} \cdot 3^{2}}=-1.778 & \frac{2^{10}}{2^{2} \cdot 3^{2} \cdot 4^{2} \cdot 5^{2}}=0.071
\end{array}
$$

$\mathrm{NO}_{\mathrm{i}}$ - Equation (103) - This is one term where powers advance $2,6,10$, etc. and with S terms

NOTE - for this rerm, there is a twpo in Stokes. It indicates Si terms advanced 1, 2, 3 but they do not in the NO' sequence (there they go $1,3,5, \ldots$ ). Using the corrected version for NO using S1, S3, S5, ... leads to answers identical to the Stokes table on $\mathrm{p}$. 34. The results match for all values of $\mathrm{m}$ given

$$
\mathrm{NO}(\mathrm{m}, \mathrm{j}):=\begin{array}{llll}
\left(\prod_{\mathrm{k}=1}^{2 \cdot j-1} \mathrm{k}^{2}\right) \cdot(2 \cdot \mathrm{j}-1)^{-1} & \mathrm{NO}(2,1)=4 & \mathrm{NO}(2,2)=-9.778 & \mathrm{NO}(2,3)=0.812 \\
\frac{2^{2} \cdot \mathrm{S}(1)}{1}=4 & \frac{2^{6} \cdot \mathrm{S}(2)}{2^{2} \cdot 3}=-8 & \frac{2^{10} \cdot \mathrm{S}(3)}{2^{2} \cdot 3^{2} \cdot 4^{2} \cdot 5}=0.652
\end{array}
$$

$N O p_{i}$ - Equation (103) - This is one term where powers advance 2, 6, 10, etc. and with S terms

$$
\begin{array}{llll}
\mathrm{NOp}(\mathrm{m}, \mathrm{j}):=\frac{(-1)^{\mathrm{j}+1} \cdot \mathrm{m}^{\mathrm{p}(\mathrm{j})} \cdot \mathrm{S}(2 \cdot \mathrm{j}-1)}{\left(\prod_{\mathrm{k}=1}^{2 \cdot j-1} \mathrm{k}^{2}\right)} & \mathrm{NOp}(2,1)=4 & \mathrm{NOp}(2,2)=-3.259 & \mathrm{NOp}(2,3)=0.162 \\
\frac{2^{2} \cdot \mathrm{S}(1)}{1}=4 & \frac{2^{6} \cdot \mathrm{S}(3)}{2^{2} \cdot 3^{2}}=-3.259 & \frac{2^{10} \cdot \mathrm{S}(5)}{2^{2} \cdot 3^{2} \cdot 4^{2} \cdot 5^{2}}=0.162
\end{array}
$$


$M e_{i}$ - Equation (103) - This is one term where powers advance $4,8,12$, etc.

$$
\begin{array}{llll}
\operatorname{Me}(m, j):=\frac{(-1)^{j+1} \cdot m^{q(j)}}{\left(\prod_{k=1}^{2 \cdot j} k^{2}\right) \cdot(2 \cdot j)^{-1}} & \frac{2^{4}}{1 \cdot 2}=8 & \frac{\operatorname{Me}(2,1)=8}{2^{2} \cdot 3^{2} \cdot 4}=-1.778 & \frac{2^{8}}{2^{2} \cdot 3^{2} \cdot 4^{2} \cdot 5^{2} \cdot 6}=0.047
\end{array}
$$

Mep - Equation (103) - This is one term where powers advance $4,8,12$, etc.

$$
\begin{array}{llll}
\operatorname{Mep}(m, j):=\frac{(-1)^{j+1} \cdot \mathrm{m}^{q(j)}}{\left(\prod_{k=1}^{2 \cdot j} \mathrm{k}^{2}\right)} & \operatorname{Mep}(2,1)=4 & \operatorname{Mep}(2,2)=-0.444 & \operatorname{Mep}(2,3)=7.901 \times 10^{-3} \\
\frac{2^{4}}{1 \cdot 2^{2}}=4 & \frac{2^{8}}{2^{2} \cdot 3^{2} \cdot 4^{2}}=-0.444 & \frac{2^{12}}{2^{2} \cdot 3^{2} \cdot 4^{2} \cdot 5^{2} \cdot 6^{2}}=7.901 \times 10^{-3}
\end{array}
$$

$\mathrm{Ne}_{\mathrm{i}}$ - Equation (103) - This is one term where powers advance $4,8,12$ etc. and with $S$ terms

$$
\operatorname{Ne}(\mathrm{m}, \mathrm{j}):-\frac{\left(-1 \mathrm{j}^{\mathrm{j}+1} \cdot \mathrm{m}^{\mathrm{q}(\mathrm{j})} \cdot \mathrm{S}(2 \cdot \mathrm{j})\right.}{\left(\prod_{\mathrm{k}=1}^{2 \cdot j} \mathrm{k}^{2}\right) \cdot(2 \cdot \mathrm{j})^{-1}} \quad \begin{array}{llll}
\mathrm{Ne}(2.1)-12 & \mathrm{Ne}(2.2)--3.704 & \mathrm{Ne}(2.3)-0.116 \\
\frac{2^{4} \cdot \mathrm{S}(2)}{1 \cdot 2}=12 & \frac{2^{8} \cdot \mathrm{S}(4)}{2^{2} \cdot 3^{2} \cdot 4}=-3.704 & \frac{2^{12} \cdot \mathrm{S}(6)}{2^{2} \cdot 3^{2} \cdot 4^{2} \cdot 5^{2} \cdot 6}=0.116
\end{array}
$$

Nep - Equation (103) - This is one term where powers advance 4, 8, 12, etc. and with $S$ terms

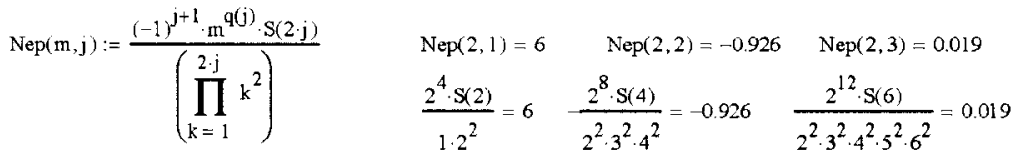

Now add up the individual terms to get the terms needed for the next step (add $S$ for sum).

$$
\begin{array}{ll}
\operatorname{MoS}(m, N):=\sum_{j=1}^{N} \operatorname{Mo}(m, j) & \operatorname{MopS}(m, N):=\sum_{j=1}^{N} \operatorname{Mop}(m, j) \\
\operatorname{NOS}(m, N):=\sum_{j=1}^{N} \operatorname{No}(m, j) & \operatorname{NopS}(m, N):=\sum_{j=1}^{N} \operatorname{Nop}(m, j) \\
\operatorname{MeS}(m, N):=\sum_{j=1}^{N} \operatorname{Me}(m, j) & \operatorname{MepS}(m, N):=\sum_{j=1}^{N} \operatorname{Mep}(m, j) \\
\operatorname{NeS}(m, N):=\sum_{j=1}^{N} \operatorname{Ne}(m, j) & \operatorname{NepS}(m, N):=\sum_{j=1}^{N} \operatorname{Nep}(m, j) \\
\operatorname{garnma}:=\gamma \text { float } \rightarrow 0.57721566490153286061 & L(m):=\ln (m)+\text { gamma }
\end{array}
$$


Create $k_{\text {Full }}$ as a single term using various product series. The desired terms $k$ and $k^{\prime}$ will then result as the real and imaginary parts of this term, respectively.

$\operatorname{KPll}(m, N):=-L(m) \cdot \operatorname{MOS}(m, N)+\frac{\pi}{4} \cdot \operatorname{MeS}(m, N)-\frac{1}{2} \cdot \operatorname{MOpS}(m, N)+\operatorname{NOS}(m, N)$

$\operatorname{KPt} 2(m, N):=\frac{\pi}{4} \cdot \operatorname{MOS}(m, N)+L(m) \cdot \operatorname{MeS}(m, N)-\frac{1}{2} \cdot(1-\operatorname{MepS}(m, N))-\operatorname{NeS}(m, N)$

$\mathrm{KPt} 3(\mathrm{~m}, \mathrm{~N}):=\frac{\pi}{4} \cdot \mathrm{MOpS}(\mathrm{m}, \mathrm{N})+\mathrm{L}(\mathrm{m}) \cdot(\mathrm{I}-\mathrm{MepS}(\mathrm{m}, \mathrm{N}))+\mathrm{NepS}(\mathrm{m}, \mathrm{N})$

$\mathrm{KPt} 4(\mathrm{~m}, \mathrm{~N}):=-\mathrm{L}(\mathrm{m}) \cdot \mathrm{MOpS}(\mathrm{m}, \mathrm{N})-\frac{\pi}{4} \cdot(\mathrm{I}-\mathrm{MepS}(\mathrm{m}, \mathrm{N}))+\mathrm{NOpS}(\mathrm{m}, \mathrm{N})$

$\operatorname{KFull}(m, N):=1+\frac{2}{m^{2}} \cdot \frac{\operatorname{KPt} 1(m, N)+\operatorname{KPt} 2(m, N) \cdot \sqrt{-1}}{\operatorname{KPt} 3(m, N)+\operatorname{KPt} 4(m, N) \cdot \sqrt{-1}}$

$\mathrm{K} \_\operatorname{Re}(\mathrm{m}, \mathrm{N}):=\operatorname{Re}(\mathrm{KFull}(\mathrm{m}, \mathrm{N})) \quad$ In Stokes' paper, $\mathrm{k}$ is the real part of KFull - call it K_Re

$\mathrm{Kp} \_\operatorname{Im}(\mathrm{m}, \mathrm{N}):=\operatorname{Im}(\mathrm{KFull}(\mathrm{m}, \mathrm{N})) \quad$ In Stokes' paper, $\mathrm{k}$ is the imaginary part of KFull - call it Kp_Im

Can test several values here: $\quad \mathrm{mval}:=0.30 \quad \mathrm{~K} \operatorname{Re}(\mathrm{mval}, 40)=6.166 \quad \mathrm{Kp} \operatorname{Im}(\mathrm{mval}, 40)=9.258$

Compare these to the table printed in Stokes' paper:

\begin{tabular}{|c|c|c|c|c|c|c|c|c|}
\hline$k$ & $k^{\prime}$ & $m^{2} k$ & $\mathbf{n}^{2} k^{\prime}$ & II & k & $\overline{k^{\prime}}$ & $\mathbf{m}^{2} k$ & $\mathbf{m}^{2} k^{\prime}$ \\
\hline 0 & $\infty$ & 0 & 0 & 2.1 & 1.677 & .7822 & 7.395 & 3.450 \\
\hline -19.70 & 48.63 & 1970 & 4863 & 2.2 & 1.646 & .7421 & 7.966 & 3.592 \\
\hline .29 .166 & 16.73 & 3666 & 6691 & 2.3 & 1.618 & .7059 & 8.557 & 734 \\
\hline .36 .166 & 9.258 & .5549 & 8832 & 2,4 & 1.592 & .6730 & 9.168 & 3.877 \\
\hline 44.771 & 6.185 & 7633 & 9896 & 17 & 1.568 & 6430 & 9.799 & 4.019 \\
\hline .53 .968 & 4.567 & 9920 & 1.142 & 2.6 & 1.546 & .6154 & 10. & 4. \\
\hline .63 .445 & 3.589 & 1.240 & 1.292 & 2.7 & 1.526 & 5902 & 11. & \\
\hline-73.082 & 2.936 & 1.510 & & 3 & & .5669 & 11 & \\
\hline .82 .812 & 2.477 & 1.800 & 1.5 & 2. & 39 & .5453 & 12. & 4.5 \\
\hline 92.604 & 2 & 2.1 & & 3. & & 5253 & 13. & \\
\hline 1.02 .439 & 1.876 & 2.439 & & 3. & 457 & 5068 & & 4. \\
\hline 1.1. & & & & & & & & \\
\hline 1.22 .194 & 1.503 & 3.160 & & 3. & & 47 & & 5.154 \\
\hline $\begin{array}{ll}1.32 .102 \\
\end{array}$ & & 3.552 & & 3 & 7 & 4581 & & \\
\hline 1.42 .021 & 1.250 & 3.961 & & 3. & 405 & 4439 & 17 & 5.437 \\
\hline 1.51 .951 & & 9 & & 3. & & .4305 & & \\
\hline 1.61 .891 & & 4.841 & & 3. & & 41 & & \\
\hline 1.71 .838 & 9965 & & & 5 & & 4060 & & \\
\hline 1.81 .791 & & 5.804 & & 3. & & 3948 & & \\
\hline \begin{tabular}{l|l}
1.9 & 1.749
\end{tabular} & & & & 4.0 & 1.354 & 3841 & 21.67 & 6.14 \\
\hline 2.01 .711 & 8268 & 6.845 & p.sur & $\infty$ & 1 & & $\infty$ & \\
\hline
\end{tabular}

Now compare the tables side by side to ensure good match:
$\mathrm{p}:=1 . .40$
$A_{\mathrm{p}, 1}:=K_{-} \operatorname{Re}(0.1 \cdot \mathrm{p}, 40)$
$A_{p, 2}:=K_{p} \_\operatorname{Im}(0.1 \cdot \mathrm{p}, 40)$
$\mathrm{A}_{0,0}:=0$
$A_{0,1}:=\infty$
$\mathrm{A}_{0,2}:=\infty$ 


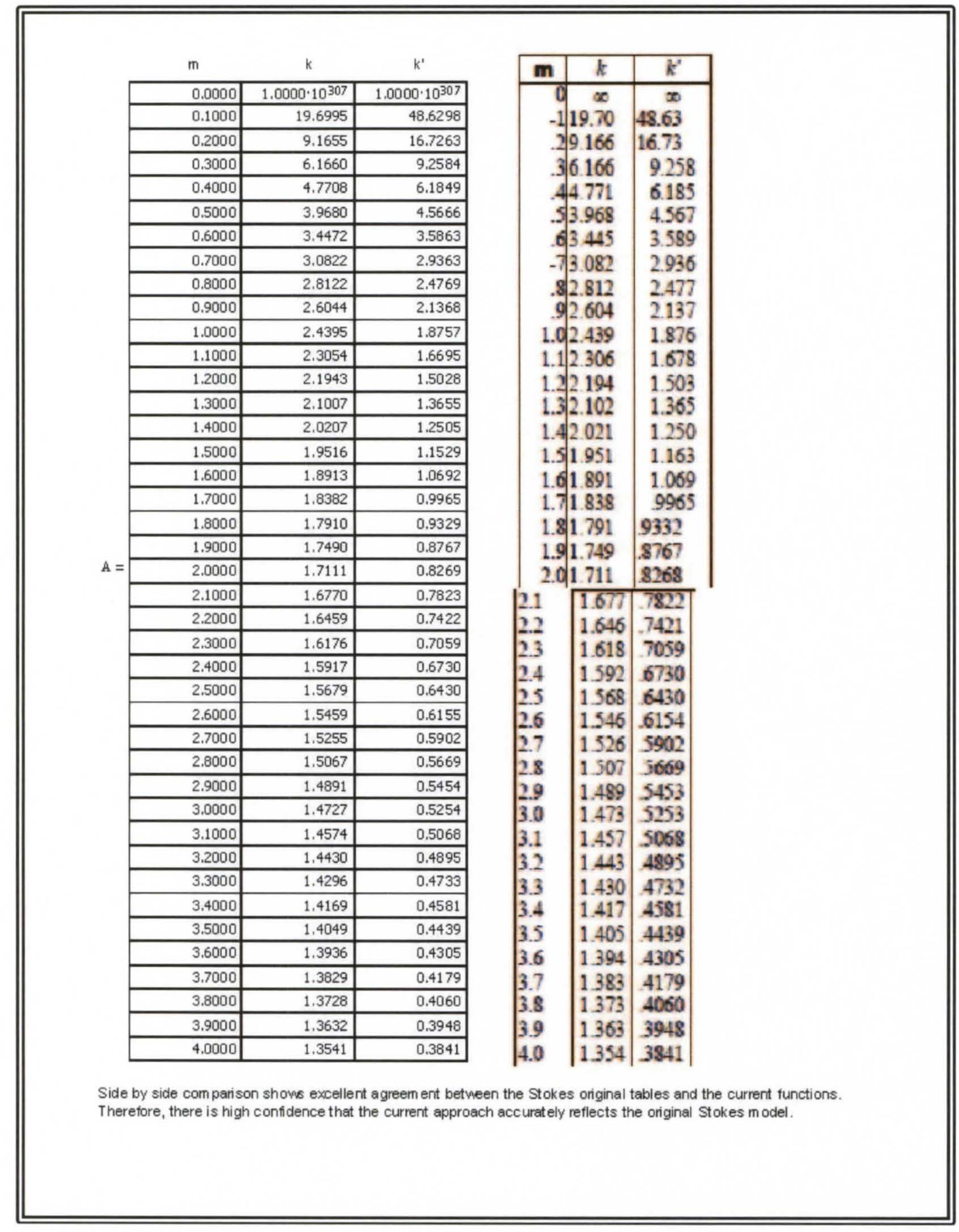


Now consider the development of a curve fit of $k$ and $k$ '. This will be based on two approximations that Stokes noted in his original paper for very small values of $m$ and large values of $m$. The region in between is where the current curve fit will be pursued.

The beginning of the table can be approximated as:

When $\mathbf{m}$ is very small, we may neglect the powers of $\mathbf{m}$ in the numerator and denominator of the fraction in the right-hand member of equation (105), retaining only the logarithms and the constant terms. We thus get

$$
k+\sqrt{-1} k=1-\frac{m^{-1} \sqrt{-1}}{L-\frac{1}{6} \pi \sqrt{-1}}
$$

whence

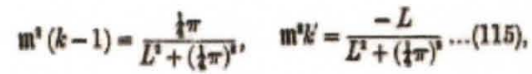

Lets try this as an approximation (letter $\mathrm{S}=\mathrm{small}$ for $\mathrm{small}$ values of $\mathrm{m}$ )

$$
\begin{aligned}
& \mathrm{kSapp}(\mathrm{m}):=\frac{\pi}{4 \cdot \mathrm{m}^{2} \cdot\left[\mathrm{L}(\mathrm{m})^{2}+\left(\frac{\pi}{4}\right)^{2}\right]}+1 \quad \mathrm{kpSapp}(\mathrm{m}):=\frac{-\mathrm{L}(\mathrm{m})}{\mathrm{m}^{2}\left[\mathrm{~L}(\mathrm{~m})^{2}+\left(\frac{\pi}{4}\right)^{2}\right]} \\
& \mathrm{j}:=0.40 \quad \mathrm{mS}_{\mathrm{j}}:=0^{-4+\frac{\mathrm{j}}{8}} \quad \text { Small values of } \mathrm{m} \text {, evenly spaced in log } \mathrm{m} \text { between } 0.0001 \text { and } 10
\end{aligned}
$$

$\mathrm{AS}_{\mathrm{j}, 0}:=\mathrm{mS}_{\mathrm{j}}$

$$
\begin{aligned}
& \mathrm{AS}_{\mathrm{j}, 1}:=\mathrm{K} \operatorname{Re}\left(\mathrm{mS}_{\mathrm{j}}, 40\right) \\
& \mathrm{AS}_{\mathrm{j}, 3}:=\mathrm{kSapp}\left(\mathrm{mS}_{\mathrm{j}}\right) \\
& \mathrm{AS}_{\mathrm{j}, 5}:=100 \cdot \frac{\mathrm{AS}_{\mathrm{j}, 3}-\mathrm{AS}_{\mathrm{j}, 1}}{\mathrm{AS} \mathrm{j}_{\mathrm{j}, 1}}
\end{aligned}
$$$$
\mathrm{AS}_{\mathrm{j}, 2}:=\mathrm{Kp}_{\_} \operatorname{Im}\left(\mathrm{mS}_{\mathrm{j}}, 40\right)
$$

Stokes Equations

$\operatorname{AS}_{j, 4}:=\operatorname{kpSapp}\left(\mathrm{mS}_{\mathrm{j}}\right)$

Approx Equations

$\mathrm{AS}_{\mathrm{j}, 6}:=100 \cdot \frac{\mathrm{AS}_{\mathrm{j}, 4}-\mathrm{AS}_{\mathrm{j}, 2}}{\mathrm{AS}_{\mathrm{j}, 2}}$

Percent Error 


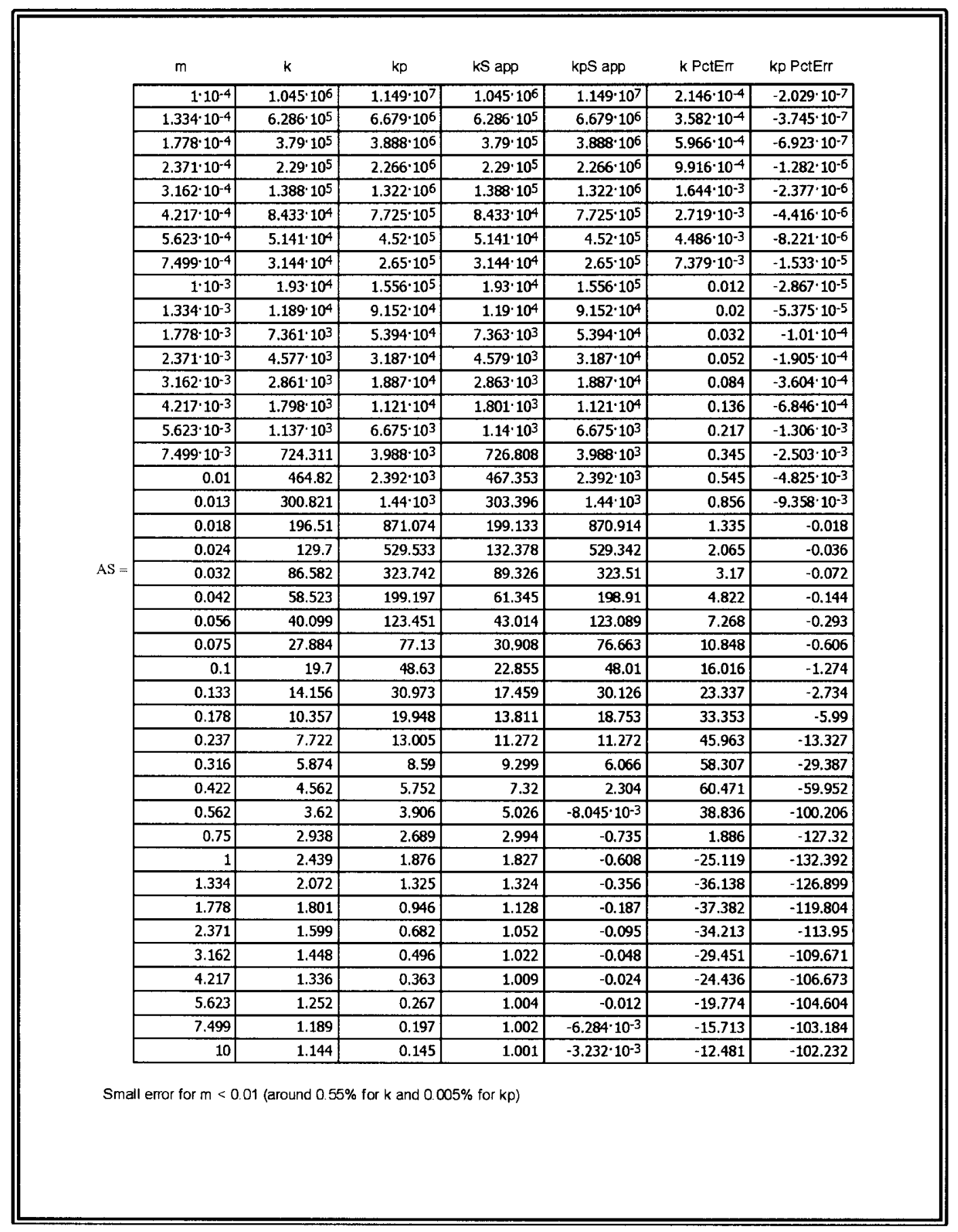




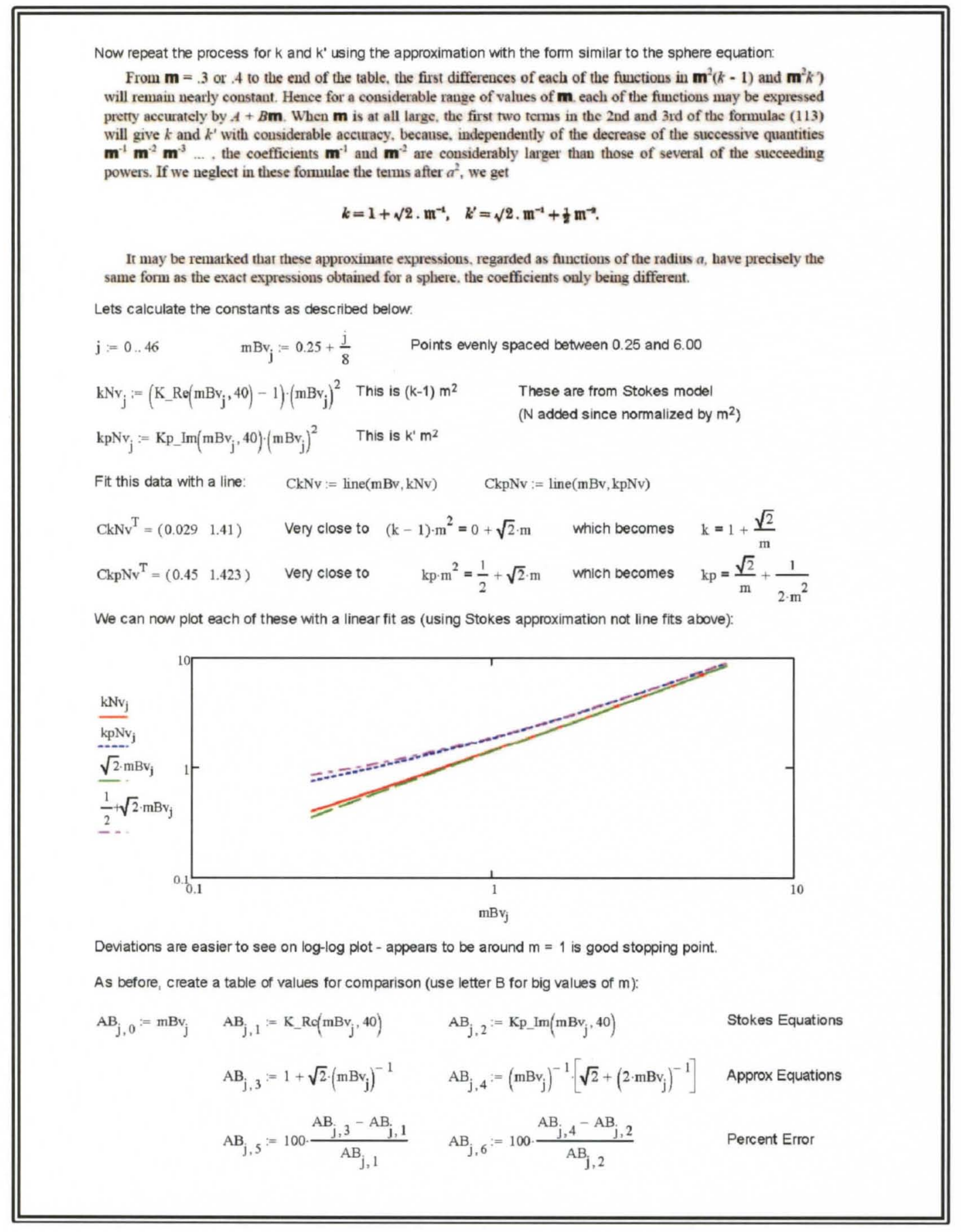




\begin{tabular}{|c|c|c|c|c|c|c|c|c|}
\hline \multirow{47}{*}{$\mathrm{AB}=$} & 0.25 & 7.333 & 12.04 & \begin{tabular}{ll|}
6.657 \\
\end{tabular} & 13.657 & -9.22 & 13.427 & \multirow{47}{*}{$\begin{array}{l}\text { Columns from left to right are: } \\
m k k^{\prime} k B a k^{\prime} B a k P c t k^{\prime} P c t \\
\text { where } k B a \text { and } k^{\prime} B a \text { are the } \\
\text { approximations from Stokes } \\
\text { paper for large } m(>0.30) \\
\text { Results are quite good for } m>2 \\
(-0.236 \% \text { and } 0.634 \% \text { error). }\end{array}$} \\
\hline & \begin{tabular}{|c|}
0.375 \\
\end{tabular} & 5.044 & \begin{tabular}{|c|}
6.763 \\
\end{tabular} & 4.771 & 7.327 & \begin{tabular}{|c|}
-5.41 \\
\end{tabular} & 8.342 & \\
\hline & 0.5 & 3.968 & 4.567 & 3.828 & 4.828 & -3.518 & 5.734 & \\
\hline & 0.625 & 3.344 & 3.4 & 3.263 & 3.543 & -2.443 & 4.197 & \\
\hline & 0.75 & 2.938 & 2.688 & 2.886 & 2.775 & -1.777 & 3.21 & \\
\hline & 0.875 & 2.652 & 2.213 & 2.616 & 2.269 & -1.338 & 2.535 & \\
\hline & 1 & 2.439 & 1.876 & 2.414 & 1.914 & \begin{tabular}{r|}
-1.036 \\
\end{tabular} & 2.054 & \\
\hline & 1.125 & 2.276 & 1.625 & 2.257 & 1.652 & \begin{tabular}{l|l|}
-0.82 \\
\end{tabular} & 1.697 & \\
\hline & 1.25 & 2.146 & 1.431 & 2.131 & 1.451 & -0.661 & 1.427 & \\
\hline & 1.375 & 2.04 & 1.277 & 2.029 & 1.293 & -0.541 & 1.216 & \\
\hline & 1.5 & 1.952 & 1.153 & 1.943 & 1.165 & -0.449 & 1.048 & \\
\hline & $\begin{array}{ll}1.625 \\
\end{array}$ & 1.877 & 1.05 & 1.87 & 1.06 & -0.377 & 0.913 & \\
\hline & 1.75 & 1.814 & 0.964 & 1.808 & 0.971 & -0.32 & 0.802 & \\
\hline & 1.875 & 1.759 & 0.89 & 1.754 & 0.896 & -0.274 & 0.711 & \\
\hline & 2 & 1.711 & 0.827 & 1.707 & 0.832 & -0.236 & 0.634 & \\
\hline & 2.125 & 1.669 & 0.772 & 1.666 & \begin{tabular}{ll|}
0.776 \\
\end{tabular} & -0.205 & 0.569 & \\
\hline & 2.25 & 1.631 & 0.724 & 1.629 & 0.727 & -0.179 & 0.513 & \\
\hline & 2.375 & 1.598 & 0.681 & 1.595 & 0.684 & -0.158 & 0.465 & \\
\hline & 2.5 & 1.568 & 0.643 & 1.566 & 0.646 & -0.14 & 0.424 & \\
\hline & 2.625 & 1.541 & 0.609 & 1.539 & 0.611 & -0.124 & 0.387 & \\
\hline & 2.75 & 1.516 & 0.578 & 1.514 & 0.58 & -0.111 & 0.356 & \\
\hline & 2.875 & 1.493 & $\begin{array}{l}0.551 \\
\end{array}$ & 1.492 & 0.552 & \begin{tabular}{|c|}
-0.099 \\
\end{tabular} & 0.328 & \\
\hline & 3 & 1.473 & 0.525 & 1.471 & $\mid 0.527$ & -0.089 & 0.303 & \\
\hline & 3.125 & 1.454 & 0.502 & 1.453 & 0.504 & -0.081 & 0.281 & \\
\hline & 3.25 & 1.436 & 0.481 & 1.435 & 0.482 & \begin{tabular}{|c|}
-0.073 \\
\end{tabular} & 0.261 & \\
\hline & 3.375 & 1.42 & 0.462 & 1.419 & 0.463 & $\begin{array}{r}-0.067 \\
\end{array}$ & 0.243 & \\
\hline & 3.5 & 1.405 & 0.444 & 1.404 & 0.445 & -0.061 & 0.227 & \\
\hline & 3.625 & 1.391 & 0.427 & 1.39 & \begin{tabular}{l|l|}
0.428 \\
\end{tabular} & -0.056 & 0.213 & \\
\hline & 3.75 & 1.378 & 0.412 & 1.377 & 0.413 & -0.051 & 0.2 & \\
\hline & 3.875 & 1.366 & 0.398 & 1.365 & 0.398 & \begin{tabular}{|c|}
-0.047 \\
\end{tabular} & 0.188 & \\
\hline & 4 & 1.354 & 0.384 & 1.354 & 0.385 & \begin{tabular}{|c|}
-0.043 \\
\end{tabular} & 0.177 & \\
\hline & 4.125 & 1.343 & 0.372 & 1.343 & 0.372 & -0.04 & 0.167 & \\
\hline & 4.25 & 1.333 & \begin{tabular}{l|l|}
0.36 \\
\end{tabular} & 1.333 & 0.36 & -0.037 & 0.157 & \\
\hline & 4.375 & 1.324 & 0.349 & 1.323 & \begin{tabular}{l|l|}
0.349 \\
\end{tabular} & -0.034 & 0.149 & \\
\hline & 4.5 & 1.315 & 0.338 & 1.314 & 0.339 & -0.032 & 0.141 & \\
\hline & 4.625 & 1.306 & 0.329 & 1.306 & 0.329 & \begin{tabular}{l|}
-0.03 \\
\end{tabular} & 0.134 & \\
\hline & 4.75 & 1.298 & 0.319 & 1.298 & 0.32 & -0.028 & 0.127 & \\
\hline & 4.875 & 1.29 & 0.311 & 1.29 & 0.311 & \begin{tabular}{l|l|}
-0.026 \\
\end{tabular} & 0.121 & \\
\hline & 5 & 1.283 & 0.302 & 1.283 & 0.303 & -0.024 & 0.115 & \\
\hline & 5.125 & 1.276 & 0.295 & 1.276 & 0.295 & -0.023 & 0.11 & \\
\hline & 5.25 & 1.27 & 0.287 & 1.269 & 0.288 & -0.021 & 0.105 & \\
\hline & 5.375 & 1.263 & 0.28 & 1.263 & 0.28 & $\begin{array}{l}-0.02 \\
\end{array}$ & 0.101 & \\
\hline & 5.5 & 1.257 & 0.273 & 1.257 & 0.274 & -0.019 & 0.096 & \\
\hline & 5.625 & 1.252 & 0.267 & 1.251 & 0.267 & -0.018 & 0.092 & \\
\hline & 5.75 & 1.246 & 0.261 & 1.246 & 0.261 & -0.017 & 0.088 & \\
\hline & 5.875 & 1.241 & 0.255 & 1.241 & 0.255 & \begin{tabular}{c|}
-0.016 \\
\end{tabular} & 0.085 & \\
\hline & 6 & 1.236 & 0.249 & 1.236 & 0.25 & -0.015 & 0.081 & \\
\hline
\end{tabular}


Now lets consider the space in between the two approximations $(m<0.015$ for small $m$ approximation and $m>2$ for big $m$ approximation, with each case having less than $0.65 \%$ error throughout for both $k$ and $k^{\prime}$ ).

Note that the big value approximation worked by multiplying by $\mathrm{m}^{2}$ and then doing a line fit. Lets try a similar approach, multiplying by $\mathrm{mP}$ where $\mathrm{p}$ is some non-integer power and then using a 2 nd order fit and see what happens It turns out that different limits and powers work for $k$ and $k$ so these are done separately below.

$\mathrm{m} 0:=0.016 \quad \mathrm{mF}:=2 \quad \mathrm{a} 0:=\log (\mathrm{m} 0) \quad \mathrm{aF}:=\log (\mathrm{mF})$

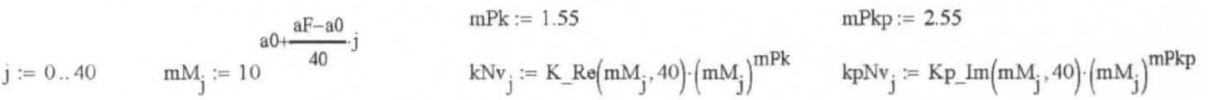

$\mathrm{k}, \mathrm{k}^{\prime}$ Multiplied By $\mathrm{m}^{\wedge} \mathrm{p}, \mathrm{m}^{\wedge} \mathrm{pk}$, Respectively

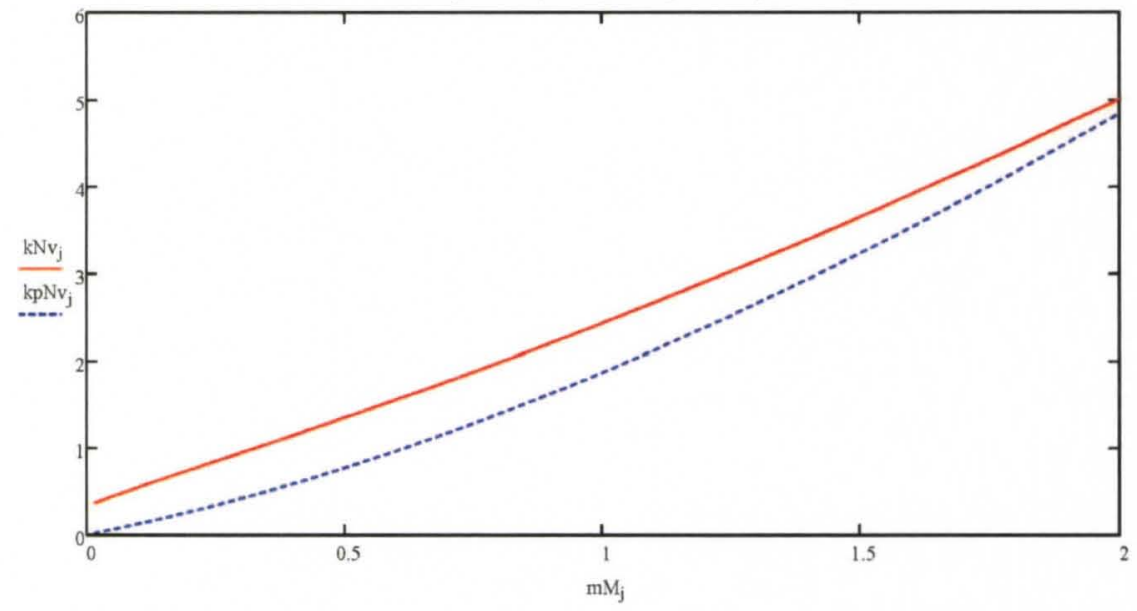

These both look very much like polynomials of 2 nd ord 3 rd order. Note that $k$ is multiplied by $m^{1.55}$ while $k^{\prime}$ is

multiplied by $m^{2.55}$. These choices were obtained by attempting to make the approach to $m=0.015$ be approximately linear in slope. Other values of $\mathrm{m}$ lead to different outcomes such as sharp downturn or upturn as approach 0.01

Now enforce the condition at $\mathrm{m}=0.01$ as follows. Subtract from $\mathrm{mM}$ the value 0.015 - this will be $\mathrm{x}$ for the polynomial curve that follows. Subtract from the data to be fit the value of the first point (the value at 0.015 ) from the small $\mathrm{m}$ approximation - this ensures that our $\mathrm{m}$ approximation is continuous. This becomes:
$\mathrm{kSO}:=\mathrm{kSapp}\left(\mathrm{mM}_{0}\right) \cdot\left(\mathrm{mM}_{0}\right)^{\mathrm{mPk}}$
$\mathrm{kpS0}:=\mathrm{kpSapp}\left(\mathrm{mM}_{0}\right) \cdot\left(\mathrm{mM}_{0}\right)^{\mathrm{mPkp}}$
$\mathrm{kSO}=0.38199$
$\mathrm{kpS0}=0.02757$
$\mathrm{kNAv}_{\mathrm{j}}:=\mathrm{kNv}_{\mathrm{j}}-\mathrm{kSO}$
$\mathrm{kpNAv}_{\mathrm{j}}:=\mathrm{kpNv}_{\mathrm{j}}-\mathrm{kpSO}$
$\mathrm{mMA}_{\mathrm{j}}:=\mathrm{mM}_{\mathrm{j}}-\mathrm{mM}_{0}$

Fit this data set with a polynomial of 3 rd order with the constant term eliminated. Hence: $\quad k F(x, c):=c_{0} \cdot x+c_{1} \cdot x^{2}+c_{2} \cdot x^{3}$

$\mathrm{k}(\mathrm{Re})$ data fit: $\quad \mathrm{cRg}:=\left(\begin{array}{l}2 \\ 0 \\ 0\end{array}\right) \quad \mathrm{cR}:=\operatorname{genfit}(\mathrm{mMA}, \mathrm{kNAv}, \mathrm{cRg}, \mathrm{kF}) \quad \mathrm{cR}^{\mathrm{T}}=\left(\begin{array}{lll}1.9755 & 0.0626 & 0.0605\end{array}\right)$

$\mathrm{K}^{\prime}(\mathrm{Im})$ data fit: $\quad \mathrm{cIg}:=\mathrm{cRg} \quad \mathrm{cI}:=\operatorname{genfit}(\mathrm{mMA}, \mathrm{kpNAv}, \mathrm{cRg}, \mathrm{kF}) \quad \mathrm{cI}^{\mathrm{T}}=\left(\begin{array}{lll}1.2185 & 0.7283 & -0.0602\end{array}\right)$ 


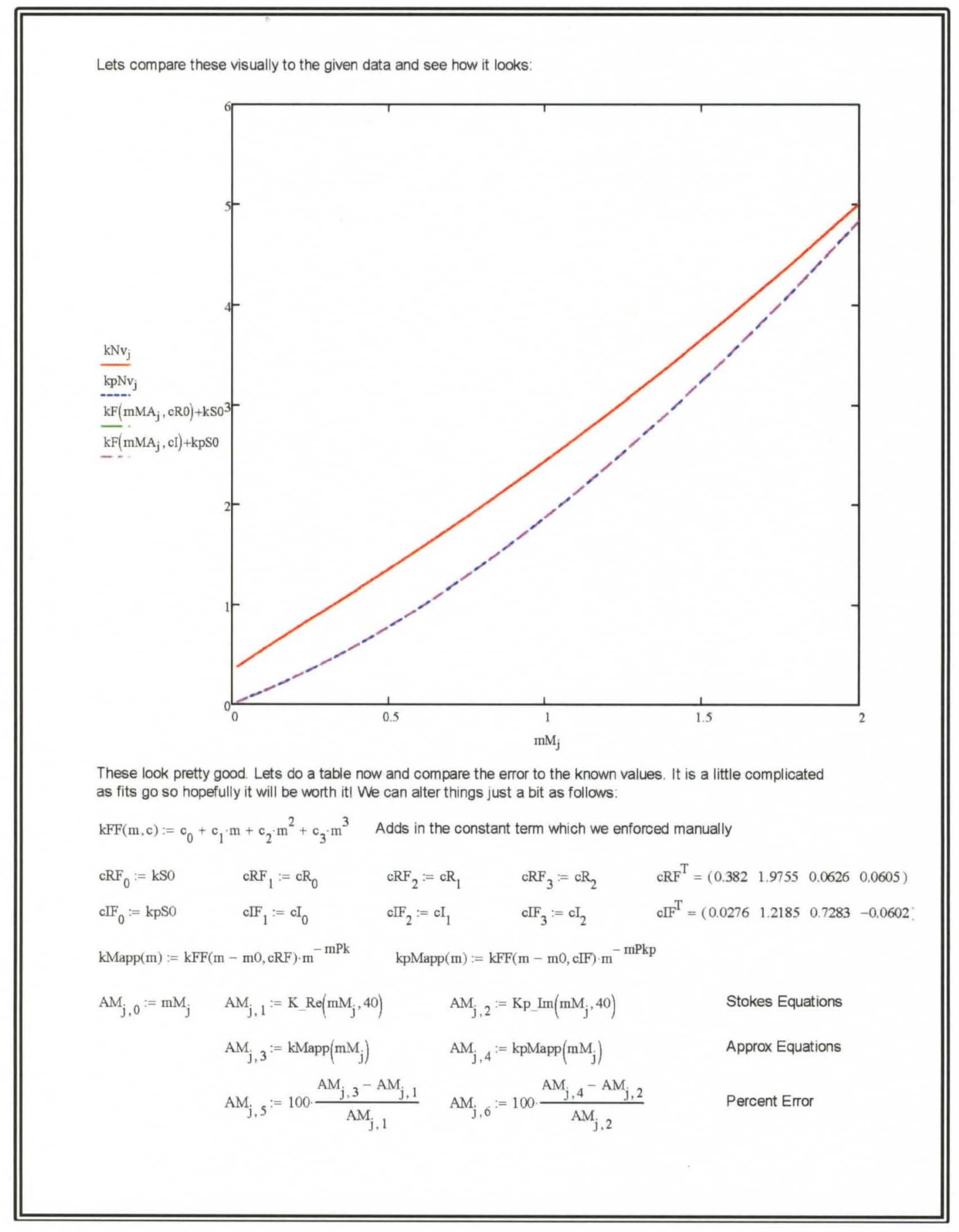




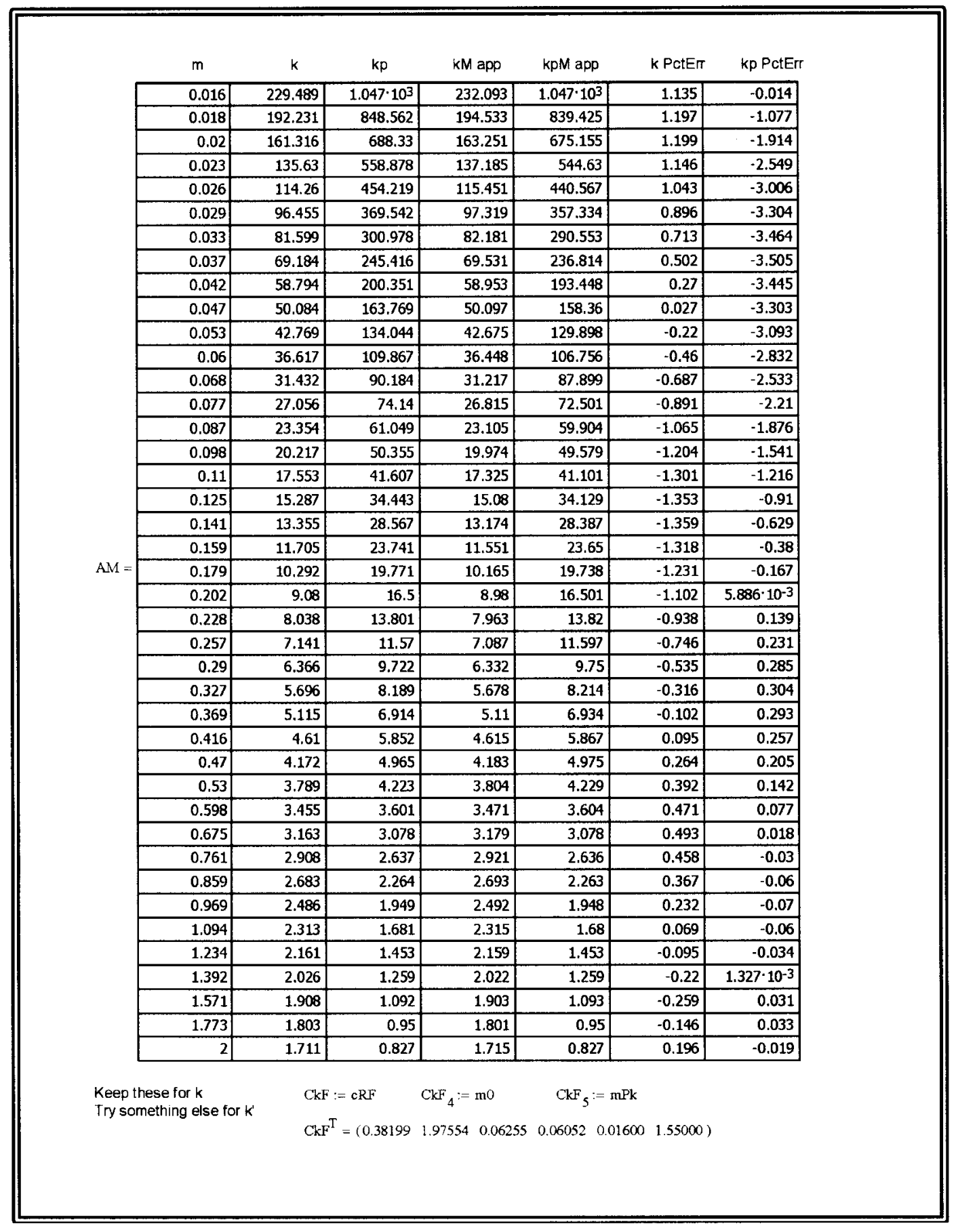


Now lets consider the space in between the two approximations $(m<0.080$ for small $m$ approximation and $m>2$ for big $m$ approximation, with each case having less than $0.65 \%$ error throughout for both $k$ and $k^{\prime}$ ).

Note that the big value approximation worked by multiplying by $\mathrm{m} 2$ and then doing a line fit. Lets try a similar approach, multiplying by $\mathrm{m}^{3}$ and then using a 2 nd order fit and see what happens.

$\mathrm{m} 0:=0.060 \quad \mathrm{mF}:=2 \quad \mathrm{a} 0:=\log (\mathrm{m} 0) \quad \mathrm{aF}:=\log (\mathrm{mF})$

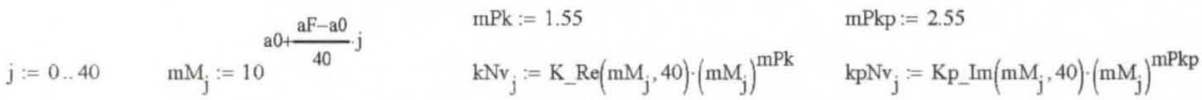

$\mathrm{k}, \mathrm{k}^{\prime}$ Multiplied By $\mathrm{m}^{\wedge} \mathrm{p}, \mathrm{m}^{\wedge} \mathrm{pk}$, Respectively

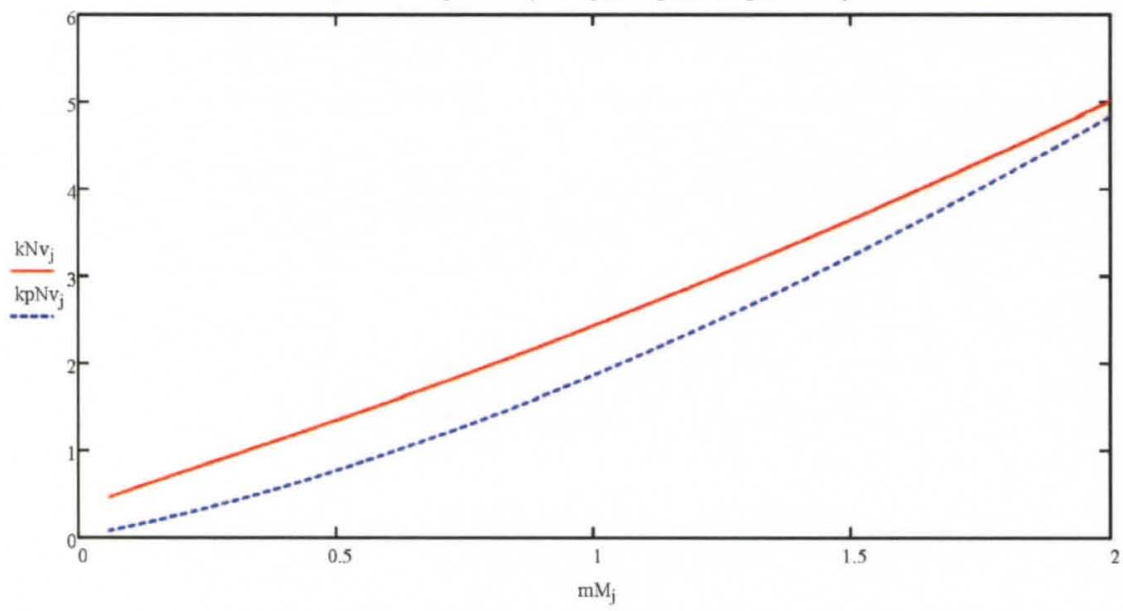

These both look very much like polynomials of 2 nd ord 3 rd order. Note that $k$ is multiplied by $m^{1.55}$ while $k^{\prime}$ is multiplied by $m^{2.55}$. These choices were obtained by attempting to make the approach to $m=0.015$ be approximately linear in slope. Other values of $m$ lead to different outcomes such as sharp downturn or upturn as approach 0.01

Now enforce the condition at $m=0.01$ as follows. Subtract from $\mathrm{mM}$ the value 0.015 - this will be $\mathrm{x}$ for the polynomial curve that follows. Subtract from the data to be fit the value of the first point (the value at 0.015 ) from the small $\mathrm{m}$ approximation - this ensures that our $\mathrm{m}$ approximation is continuous. This becomes:
$\mathrm{kS0}:=\mathrm{kSapp}\left(\mathrm{mM}_{0}\right) \cdot\left(\mathrm{mM}_{0}\right)^{\mathrm{mPk}}$
$\mathrm{kpS} 0:=\operatorname{kpSapp}\left(\mathrm{mM}_{0}\right) \cdot\left(\mathrm{mM}_{0}\right)^{\mathrm{mPkp}}$
$\mathrm{kS} 0=0.50866$
$\mathrm{kpSO}=0.08471$
$\mathrm{kNAv}_{\mathrm{j}}:=\mathrm{kNv}_{\mathrm{j}}-\mathrm{kSO}$
$\mathrm{kpNAv}_{\mathrm{j}}:=\mathrm{kpNv}_{\mathrm{j}}-\mathrm{kpSO}$
$\mathrm{mMA}_{\mathrm{j}}:=\mathrm{mM}_{\mathrm{j}}-\mathrm{mM}_{0}$

Fit this data set with a polynomial of 3rd order with the constant term eliminated. Hence: $\quad k F(x, c):=c_{0} \cdot x+c_{1} \cdot x^{2}+c_{2} \cdot x^{3}$
$k(\mathrm{Re})$ data fit: $\quad \mathrm{cRg}:=\left(\begin{array}{l}2 \\ 0 \\ 0\end{array}\right)$
$\mathrm{cR}:=$ genfit(mMA, $\mathrm{kNAv}, \mathrm{cRg}, \mathrm{kF})$
$\mathrm{cR}^{\mathrm{T}}=\left(\begin{array}{lll}1.7747 & 0.3136-0.0173\end{array}\right)$
$K^{\prime}(\mathrm{Im})$ data fit:
$\mathrm{cIg}:=\mathrm{cRg}$
$\mathrm{cI}:=$ genfit $(\mathrm{mMA}, \mathrm{kpNAv}, \mathrm{cRg}, \mathrm{kF})$
$\mathrm{cI}^{\mathrm{T}}=\left(\begin{array}{lll}1.2692 & 0.7369 & -0.0658\end{array}\right)$ 
Lets compare these visually to the given data and see how it looks:

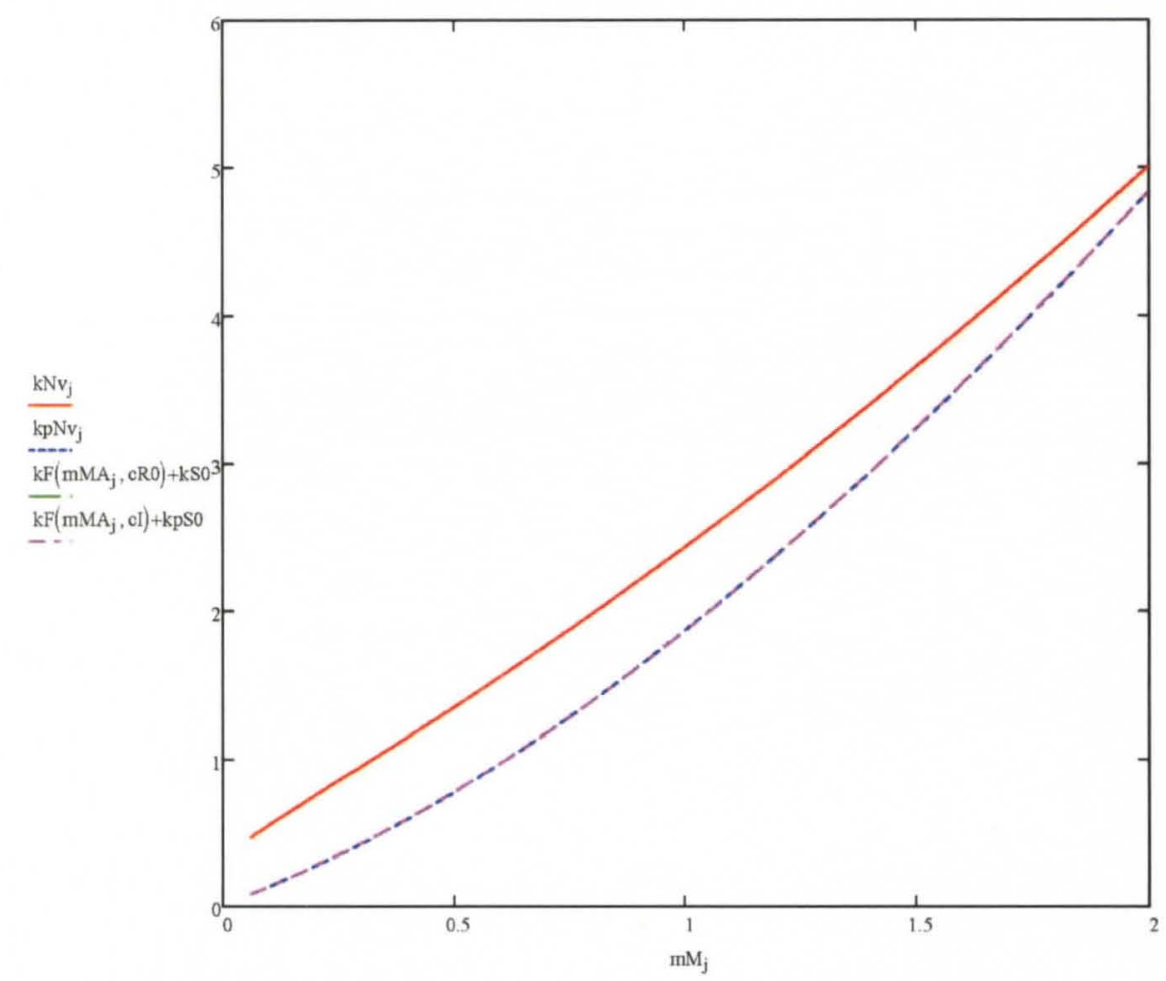

These look pretty good. Lets do a table now and compare the error to the known values. It is a little complicated as fits go so hopefully it will be worth itl We can alter things just a bit as follows:

$\mathrm{kFF}(\mathrm{m}, \mathrm{c}):=\mathrm{c}_{0}+\mathrm{c}_{1} \cdot \mathrm{m}+\mathrm{c}_{2} \cdot \mathrm{m}^{2}+\mathrm{c}_{3} \cdot \mathrm{m}^{3} \quad$ Adds in the constant term which we enforced manually
$\mathrm{cRF}_{0}:=\mathrm{kSO}$
$\mathrm{cRF}_{1}:=\mathrm{cR}_{0}$
$\mathrm{cRF}_{2}:=\mathrm{cR}_{1}$
$\mathrm{cRF}_{3}:=\mathrm{cR}_{2}$
$\mathrm{CRF}^{\mathrm{T}}=\left(\begin{array}{llll}0.5087 & 1.7747 & 0.3136 & -0.0173\end{array}\right.$
$\mathrm{cIF}_{0}:=\mathrm{kpSO}$
$\mathrm{cF}_{1}:=\mathrm{cI}_{0}$
$\mathrm{cIF}_{2}:=\mathrm{cI}_{1}$
$\mathrm{CFF}_{3}:=\mathrm{cI}_{2}$
$\mathrm{cIF}^{\mathrm{T}}=\left(\begin{array}{llll}0.0847 & 1.2692 & 0.7369 & -0.0658\end{array}\right.$

$\mathrm{kMapp}(\mathrm{m}):=\mathrm{kFF}(\mathrm{m}-\mathrm{m} 0, \mathrm{cRF}) \cdot \mathrm{m}^{-\mathrm{mPk}} \quad \mathrm{kpMapp}(\mathrm{m}):=\mathrm{kFF}(\mathrm{m}-\mathrm{m} 0, \mathrm{cIF}) \cdot \mathrm{m}^{-\mathrm{mPkp}}$
$\mathrm{AM}_{\mathrm{j}, 0}:=\mathrm{mM}_{\mathrm{j}}$
$\mathrm{AM}_{\mathrm{j}, 1}:=\mathrm{K} \operatorname{Re}\left(\mathrm{mM}_{\mathrm{j}}, 40\right)$
$\mathrm{AM}_{\mathrm{j}, 2}:=\mathrm{Kp} \operatorname{Im}\left(\mathrm{mM}_{\mathrm{j}}, 40\right)$
Stokes Equations
$\operatorname{AM}_{\mathrm{j}, 3}:=\operatorname{kMapp}\left(\mathrm{mM}_{\mathrm{j}}\right)$
$\mathrm{AM}_{\mathrm{j}, 4}:=\operatorname{kpMapp}\left(\mathrm{mM}_{\mathrm{j}}\right)$
Approx Equations
$\mathrm{AM}_{\mathrm{j}, 5}:=100 \cdot \frac{\mathrm{AM}_{\mathrm{j}, 3}-\mathrm{AM}_{\mathrm{j}, 1}}{\mathrm{AM}_{\mathrm{j}, 1}}$
$\mathrm{AM}_{\mathrm{j}, 6}:=100 \frac{\mathrm{AM}_{\mathrm{j}, 4}-\mathrm{AM}_{\mathrm{j}, 2}}{\mathrm{AM}_{\mathrm{j}, 2}}$
Percent Error 


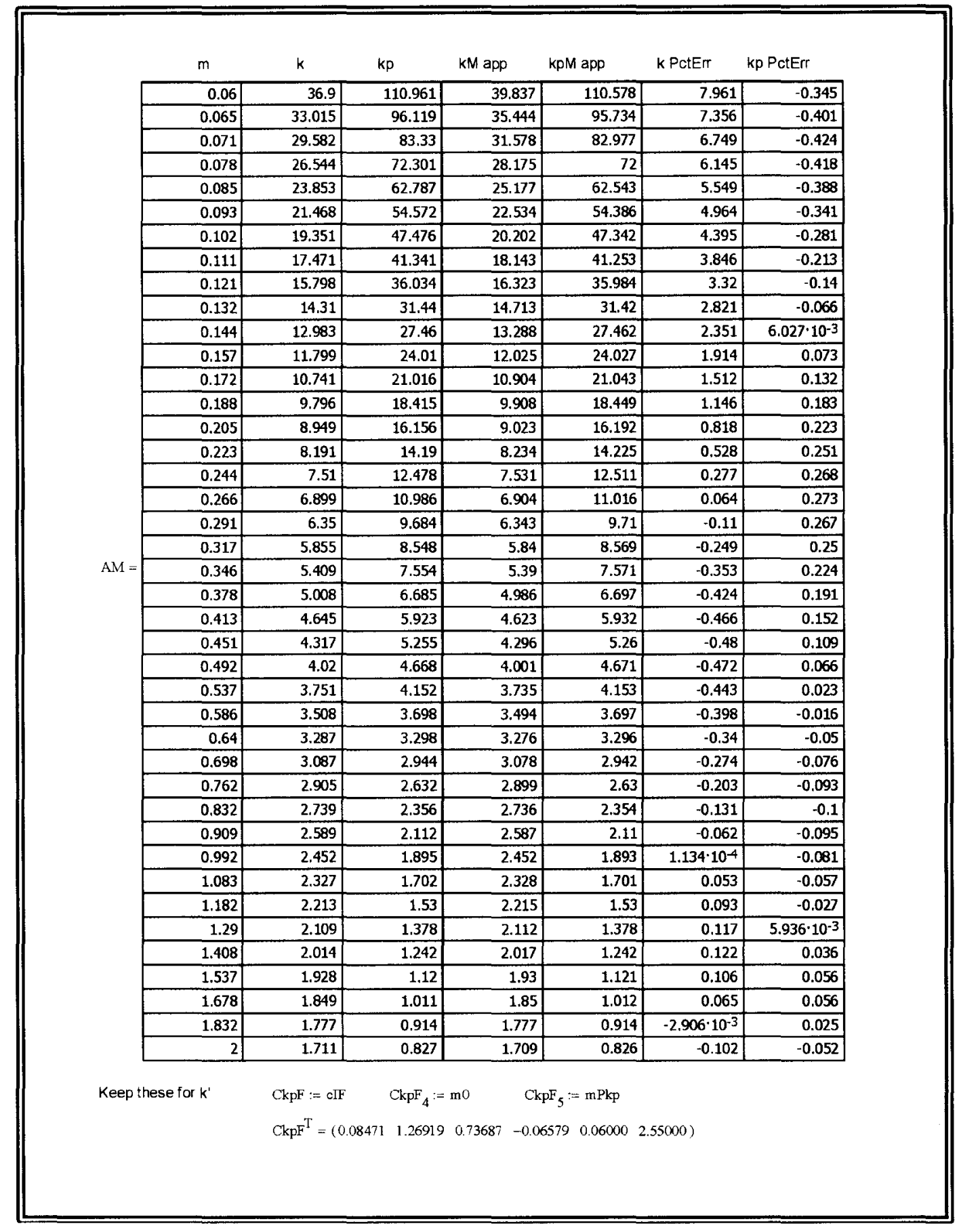




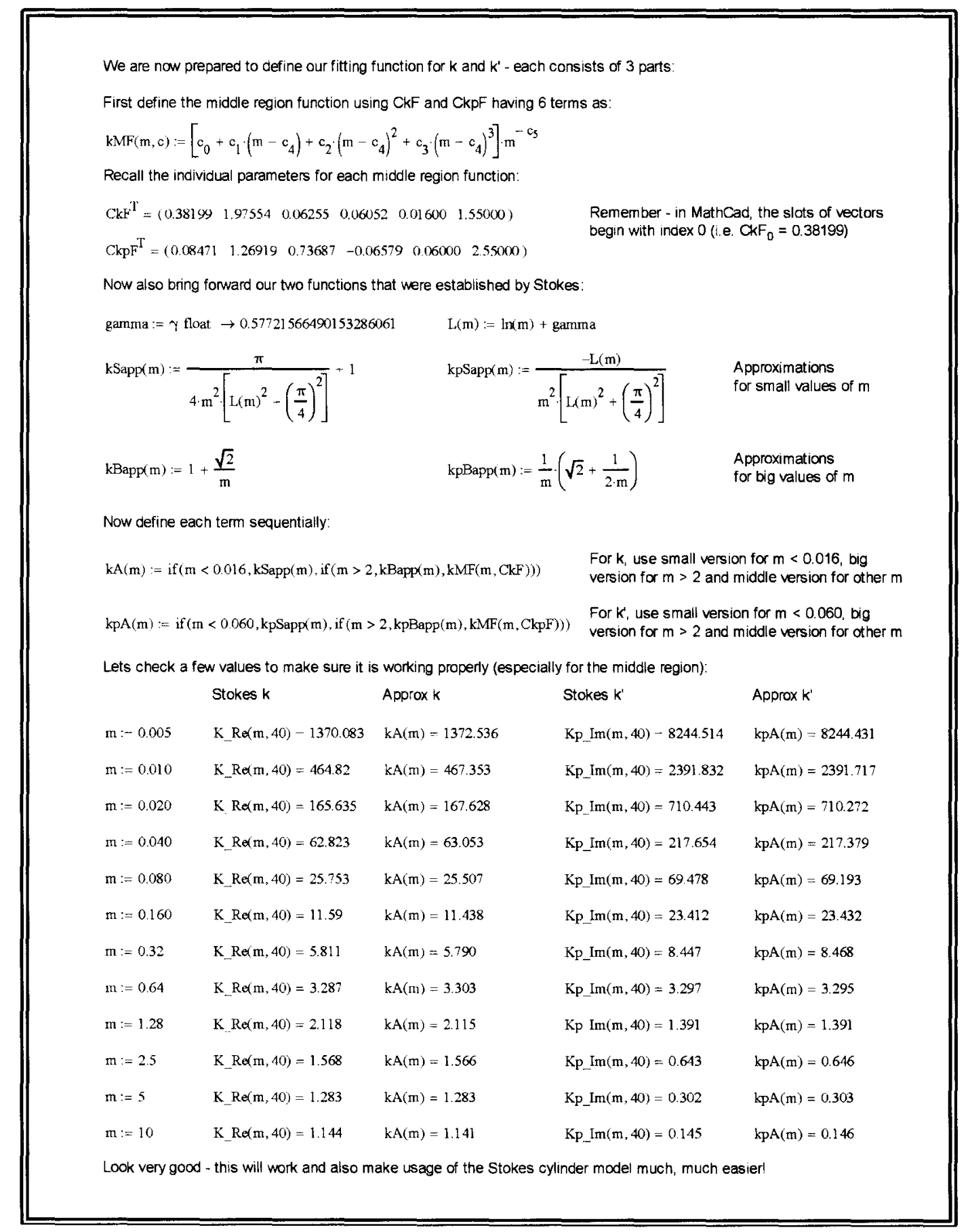


Lets end with a plot of the behavior of $k$ and $k$ across many $m$ scales and also plot the error for each term as well as a percent error between the new fitting function and the full solution developed by Stokes. For completeness, also plot the sphere approximation (i.e. $\mathrm{kB}$ for all values of $\mathrm{m}$ ):

$$
\begin{aligned}
& i:=0 . .312 \\
& m v_{i}:=10^{-3+0.0125 \cdot i}
\end{aligned}
$$

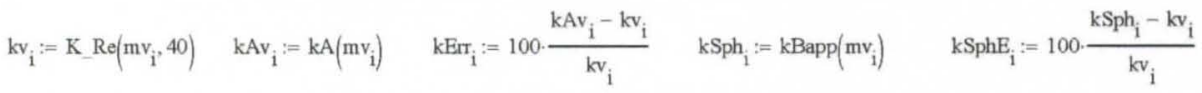
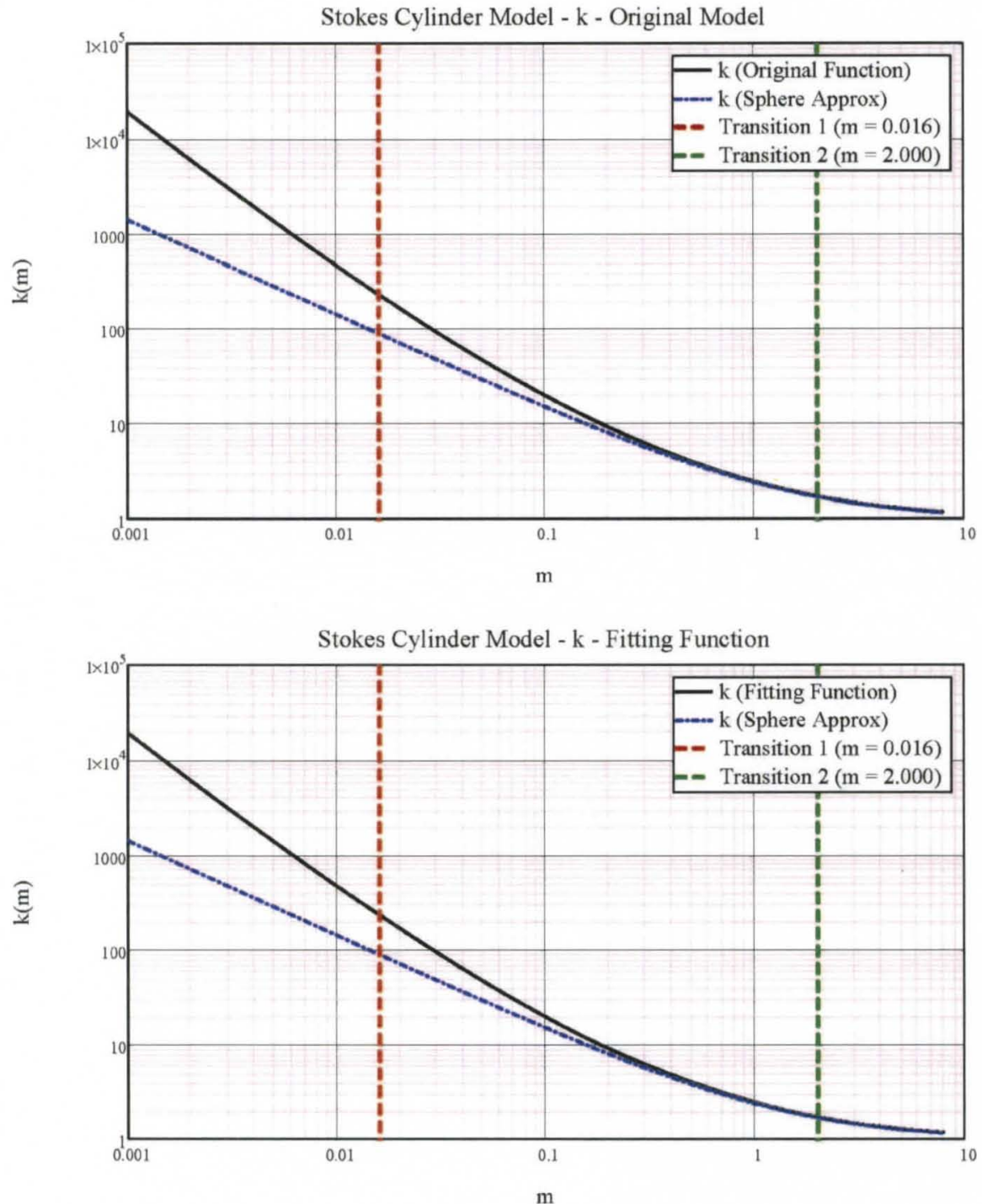

m 


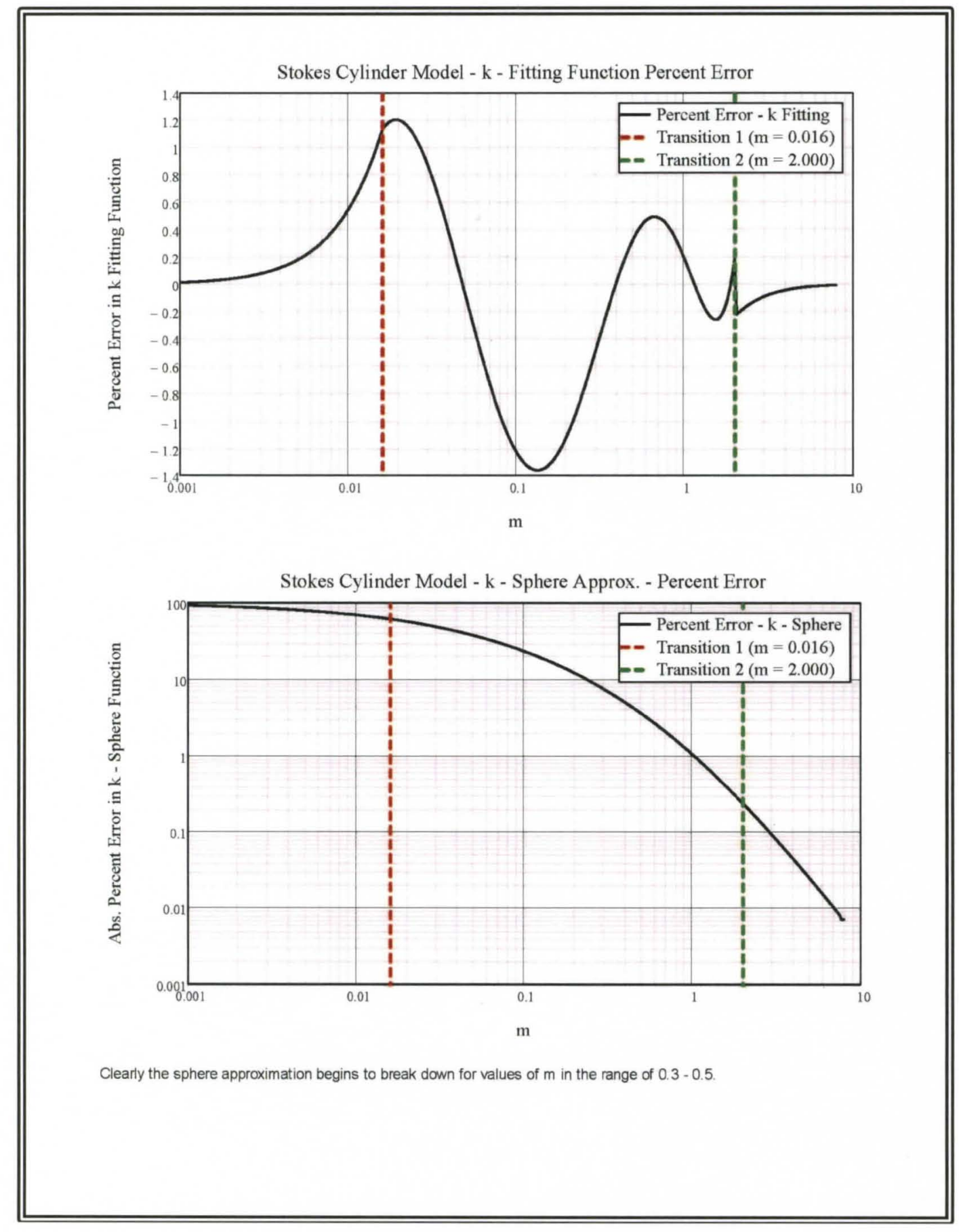




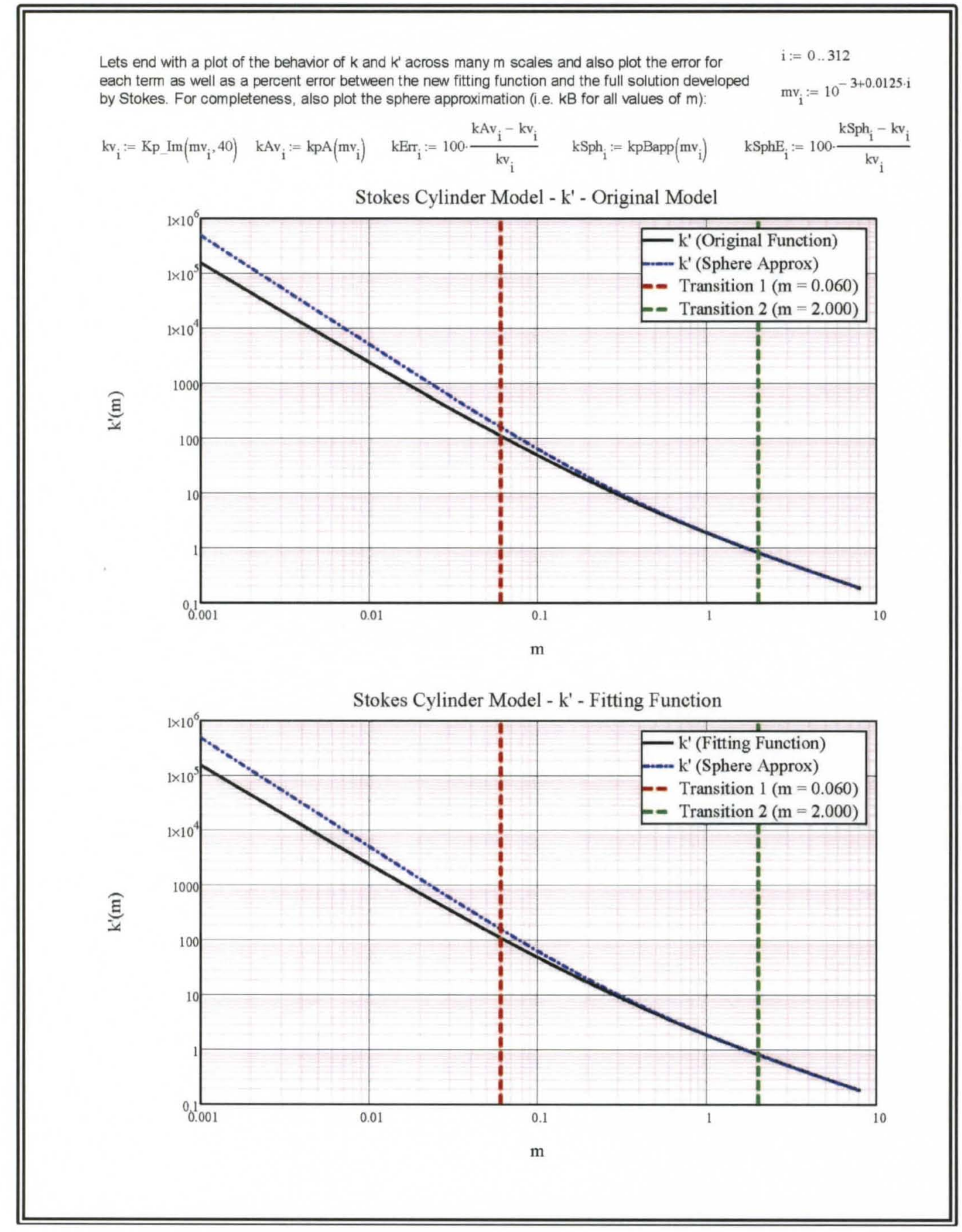




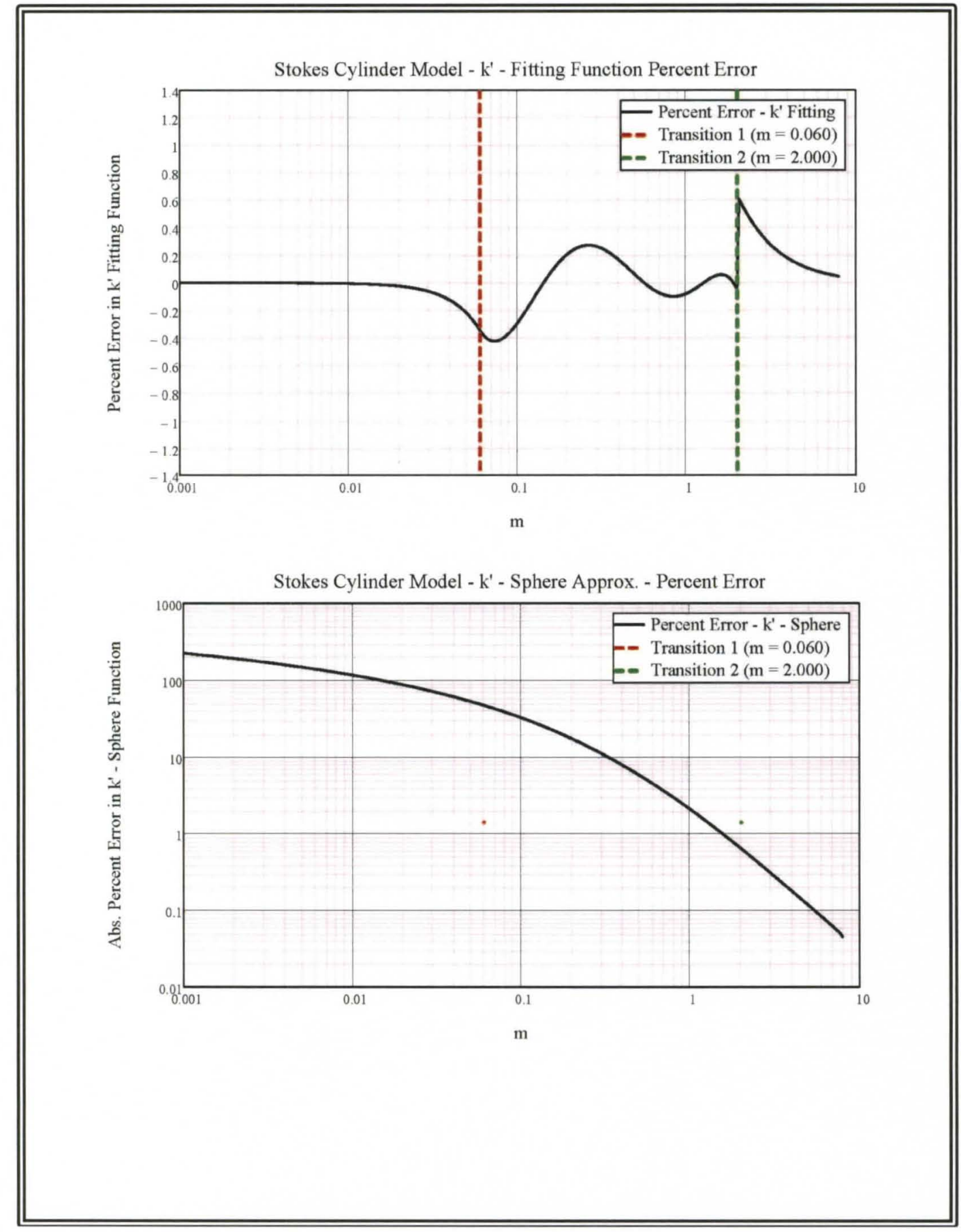




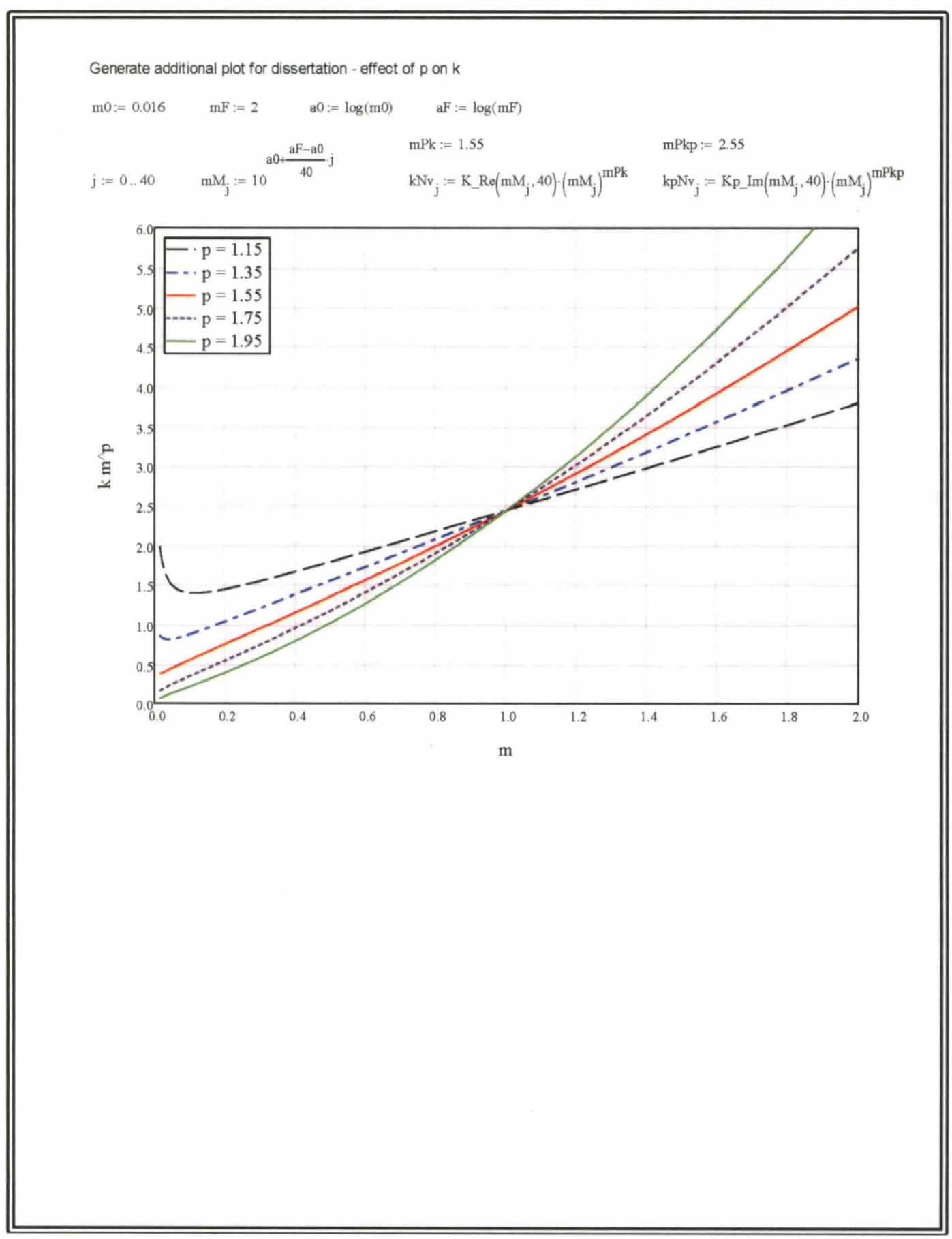




\section{APPENDIX D}

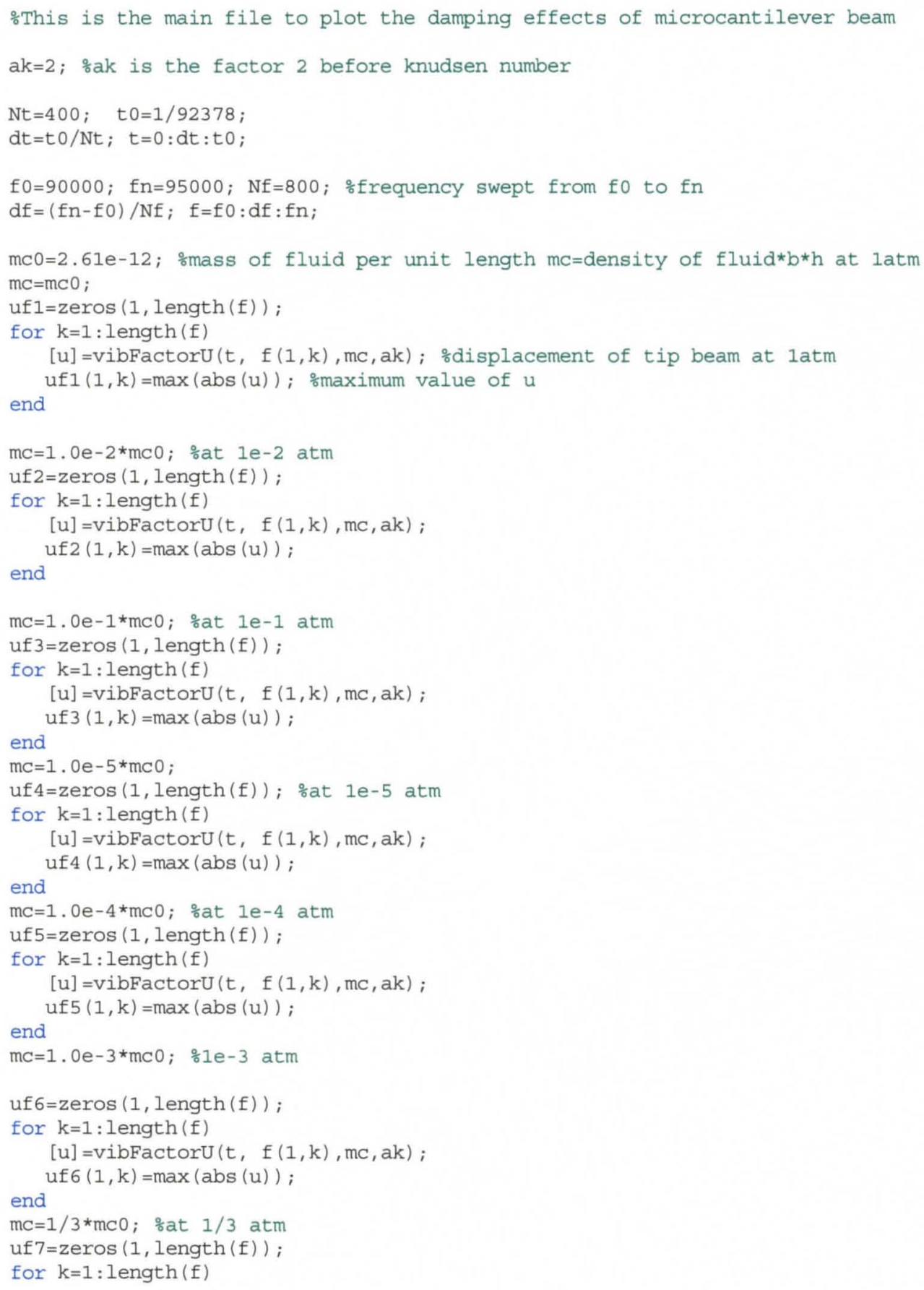




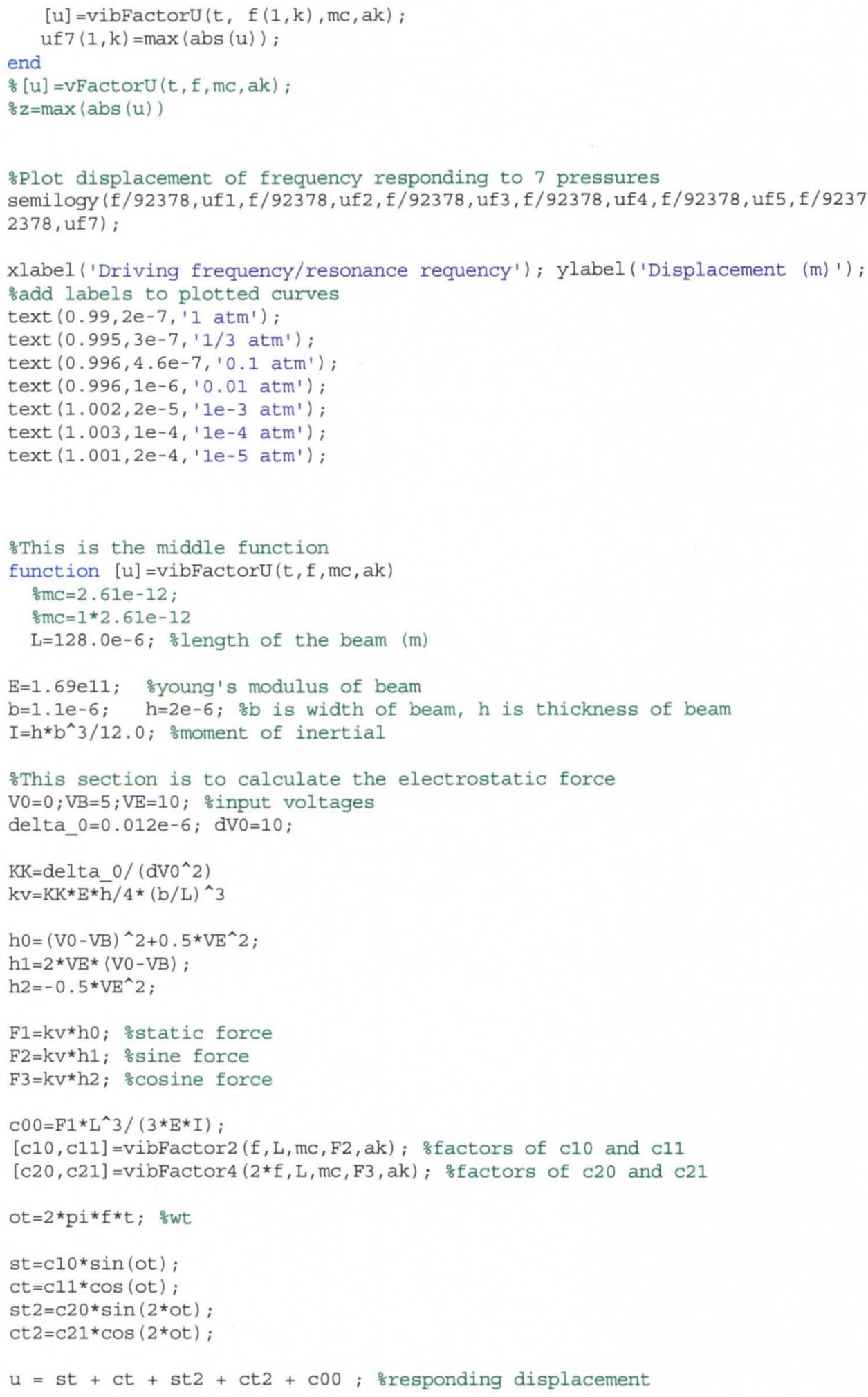




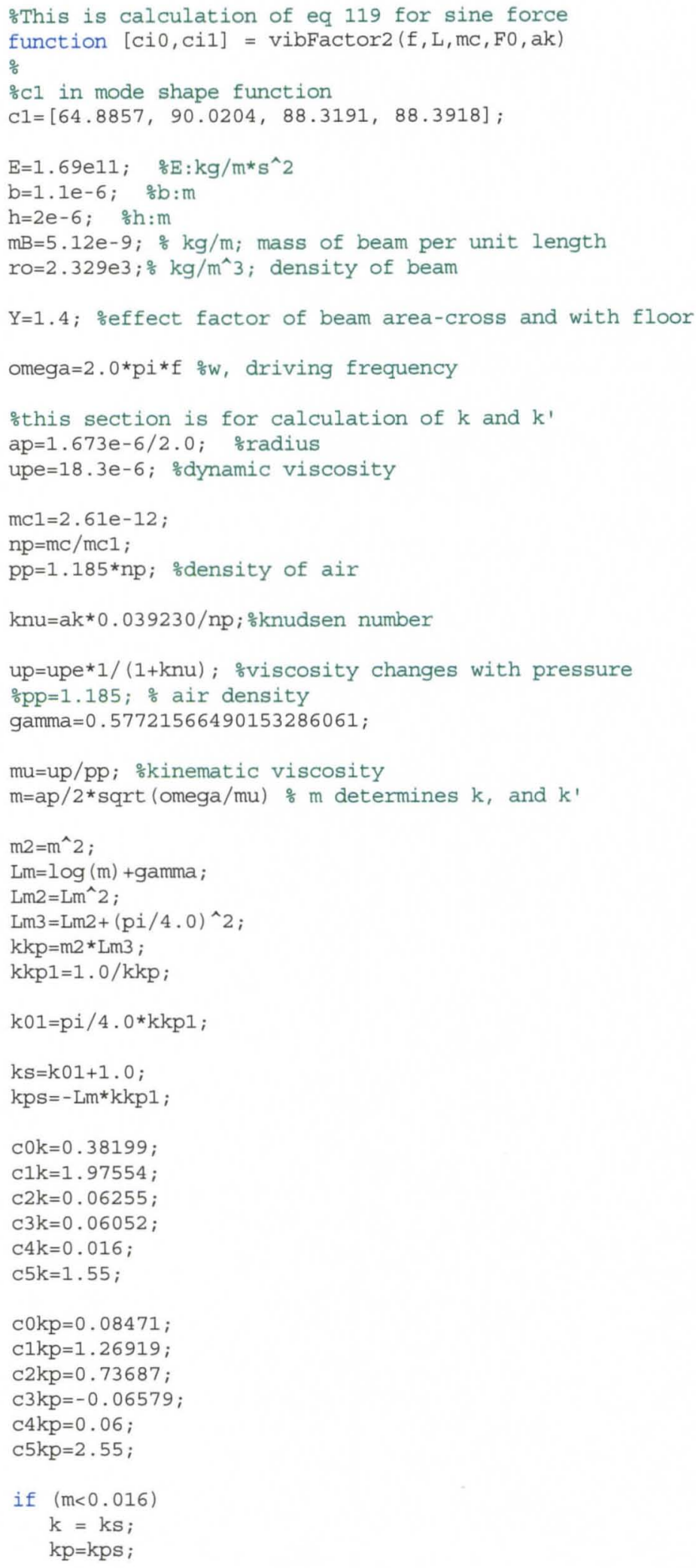




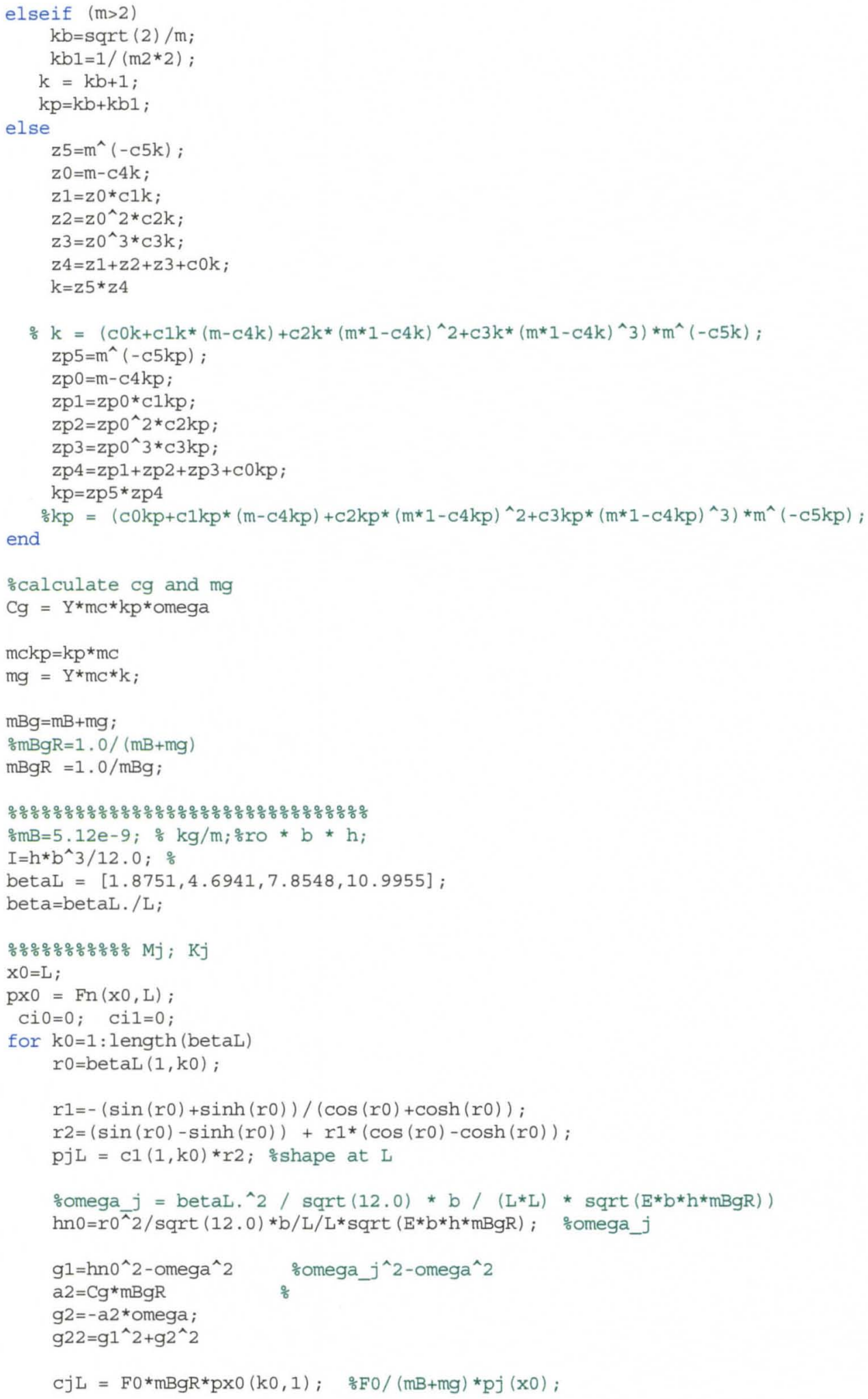




$$
\begin{aligned}
& \mathrm{Mj}=\mathrm{C} j \mathrm{~L}^{\star} \mathrm{g} 1 / \mathrm{g} 22 \text {; } \\
& \mathrm{Kj}=\mathrm{Cj} \mathrm{L}^{\star} \mathrm{g} 2 / \mathrm{g} 22 \text {; } \\
& \text { cio }=\mathrm{cio}+\mathrm{Mj}{ }^{*} \mathrm{pj} \mathrm{L}_{i} \\
& c i 1=c i 1+K j * p j L ; \\
& \text { end }
\end{aligned}
$$




\title{
CURRICULUM VITAE
}

\author{
Ni Wang \\ University of Louisville \\ Louisville, Kentucky 40292 \\ (502) $541-3440$ \\ ni.wang@louisville.edu
}

\section{PERSONAL}

\section{Education}

2007 - Present Ph.D. candidate in Mechanical Engineering, University of Louisville, Louisville, KY. Dissertation: "Improved Fabrication and Modeling of Piezoresistive Microcantilever Beams for Gas Detection and Sensing." Advisor: Dr. Roger Bradshaw, GPA = $3.87 / 4.0$.

2006 - 2007 M.S. in Mechanical Engineering, University of Louisville, Louisville, KY. Thesis: "Isothermal Physical Aging Characterization and Test Methodology for Neat and Nanotubereinforced Poly(Methyl Methacrylate) (PMMA) near the Glass Transition Temperature."

Advisor: Dr. Roger Bradshaw, GPA = 3.97 / 4.0.

$1997-2001 \quad$ B.S. in Mechanical Engineering, WuHan University of Technology, China. Thesis: "Design of double trolley quayside container cranes." Advisor: Dr. Changsheng Xu, General GPA = 3.3 / 4.0 (178 credits); Engineering Major GPA = 3.4 / 4.0 (147 credits)

\section{Experience}

2006 - Present

Research Assistant / Graduate Fellowship, Department of

Mechanical Engineering, University of Louisville, Louisville, KY 
2001-2003 Instructor, Department of Mechanical Engineering, Shanghai Maritime University. Shanghai, China.

\section{Professional Affiliations and Training}

- Society for Experimental Mechanics (SEM)

\section{Awards and Recognition}

- Recipient of The First Place in The ASME Louisville Section Student Competition (February 2011)

- Recipient of Grosscurth Engineering Fellowship, The University of Louisville (2007-2009)

- Recipient of Excellent Student Scholarship, WuHan University of Technology (1998-2000)

\section{RESEARCH / CREATIVE ACTIVITY}

\section{Research Specialties}

- Development of piezoresistive microcantilever beams for gas detection and sensing. This involves fabrication of microcantilever beams using photolithography methods in a clean-room and exploring the new access using the state of the art techniques and equipments.

- Characterization of gas damping and inertial loads on resonating microcantilevers beams using classical models and computational fluid dynamics (ANSYS CFX)

- Viscoelastic behavior of polymer films and polymer films reinforced with carbon nanotubes using static and dynamic testing with a dynamic mechanical analyzer (DMA).

\section{Publications}

$\underline{\text { Refereed Journal Articles }}$

- Guo, Y., Wang, N., Bradshaw, R.D., and Brinson, L.C. "Modeling Mechanical Aging Shift Factors in Glassy Polymers during Nonisothermal Physical Aging. I. Experiments and KAHR-ate Model Prediction," Journal of Polymer Science, Part B: Polymer Physics, 2009, 47(3), 340-352.

Full-Length Conference Papers (Presented)

- Wang, N., Alphenaar, B.W., Keynton, R.S. and Bradshaw, R.D. "Improvement of Piezoresistive Microcantilever Beams for Gas Detection and Sensing," Society for 
Experimental Mechanics Annual Conference, June 2010, Indianapolis, IN. (Wang presented at conference)

- Guo, Y., Wang, N., and Bradshaw, R.D. "Physical Aging Characterization of PPS near the Glass Transition Temperature," Society for Experimental Mechanics Annual Conference, June 2009, Albuquerque, NM. (Bradshaw presented at conference)

\section{Conference Presentations Only (No Paper Prepared)}

- Wang, N., Alphenaar, B.W., Keynton, R.S., Bradshaw, R.D. "Improvement of Piezoresistive Microcantilever beams for gas detection and sensing," Fourth Annual Nanotechnology and Nanomedicine Symposium, September 2011, Louisville, KY. (Wang presented at conference)

- Guo, Y., Wang, N., Bradshaw, R.D. "Nonisothermal physical aging of glassy polymers: experimental data and KAHR-ate model predictions," 2007 Joint ASME/ASCE/SES Conference on Mechanics and Materials, June 2007, Austin, TX. (Bradshaw presented at conference)

\section{Poster Presentations}

- Wang, N., Bradshaw, R.D. "Isothermal physical aging characterization for PMMA near the glass transition temperature," Engineering Expo, March 2007, Louisville, KY (Wang presented poster) 